\title{
Development of a Method for the $N$-Arylation of Amino Acid Esters with Aryl Triflates
}

\author{
Sandra M. King and Stephen L. Buchwald* \\ Department of Chemistry, Massachusetts Institute of Technology, \\ Cambridge, Massachusetts 02139, USA
}

Supporting Information

Table of Contents

\section{General Information}

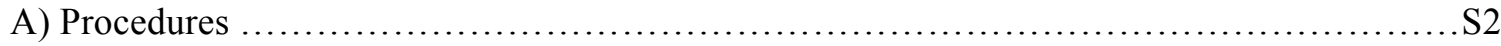

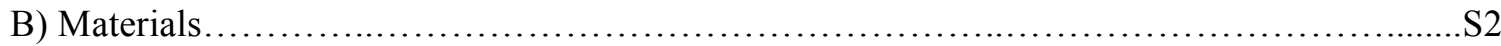

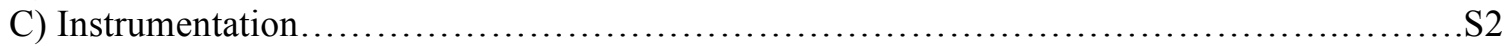

II. Experimental Procedures and Characterization Data

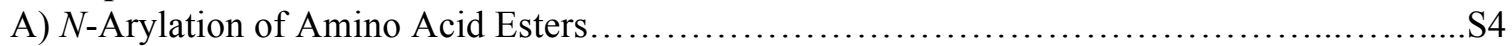

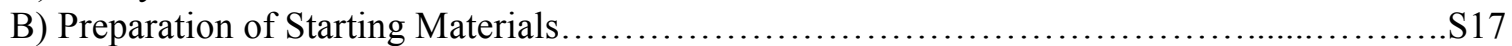

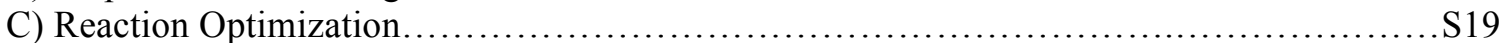

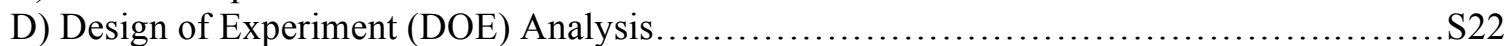

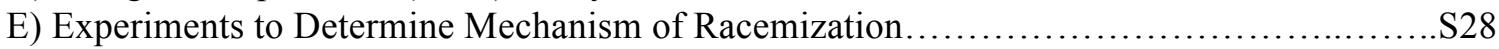

III. Catalog of Spectra

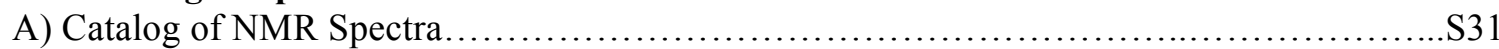

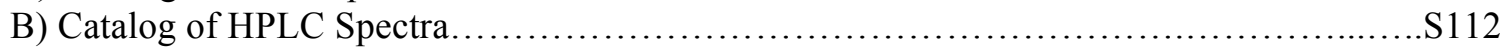

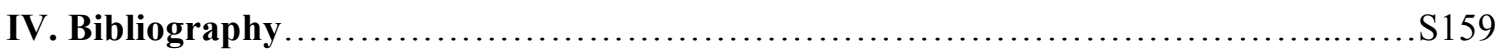




\section{General Information}

A) Procedures: All reactions were performed in oven-dried Fisher Scientific $20 \AA \sim 125 \mathrm{~mm}$ screw-cap tubes (Cat. No. 14-959-37A) using Thermo Scientific PTFE/silicon septa (Cat. No. B7995-18), unless otherwise noted. Intermediates were purified using a Biotage ${ }^{\circledR}$ Isolera system, employing polypropylene cartridges preloaded with silica gel (Silicycle SiliaFlash ${ }^{\circledR}$ F60 silica gel) or with new Biotage ${ }^{\circledR}$ SNAP cartridges. Samples were eluted using a flow rate of 18-50 $\mathrm{mL} / \mathrm{min}$, with detection by UV $(254 \mathrm{~nm})$. Analytical thin-layered chromatography (TLC) was performed using glass plates pre-coated with silica gel $(0.25 \mathrm{~mm}, 60 \AA$ pore size $)$ impregnated with a fluorescent indicator $(254 \mathrm{~nm})$. TLC plates were visualized by exposure to ultraviolet light (UV) and/or submersion in aqueous ceric ammonium molybdate solution (CAM), or aqueous potassium permanganate solution $\left(\mathrm{KMnO}_{4}\right)$.

B) Materials: Commercial solvents and reagents were purchased from Aldrich Chemical Company, Strem Chemicals, Acros Organics, Alfa Aesar, TCI America, Combi Blocks, Oakwood Chemical, Matrix Scientific, and Chem-Impex and used as received with the following exceptions. $t$-BuBrettPhos (L1) was a gift from Aldrich. Anhydrous 1,4-dioxane and 2methyltetrahydrofuran were purchased from Aldrich Chemical Co. in Sure-Seal ${ }^{\mathrm{TM}}$ bottles and used as received. THF and $\mathrm{CH}_{2} \mathrm{Cl}_{2}$ were purchased from J.T. Baker in CYCLE-TAINER® solvent-delivery kegs and vigorously purged with argon for $2 \mathrm{~h}$, followed by passing it under argon pressure through two packed columns of neutral alumina. Cesium carbonate, sodium tertbutoxide, sodium phenoxide, and potassium phosphate were stored in a nitrogen-filled glovebox. Small quantities of cesium carbonate were stored on the bench in a desiccator for up to one week. Unless otherwise noted, amino acid ester free bases were prepared from the corresponding hydrochloride salt by washing with $10 \%$ aqueous sodium carbonate. Precatalyst $\mathbf{P 3}$ was prepared by a modified literature procedure (vide infra). ${ }^{1}$ Precatalyst $\mathbf{P 1},{ }^{2}$ precatalyst $\mathbf{P 2},{ }^{1} \mathrm{~N}$-methyl-2aminobiphenylpalladium methanesulfonate dimer (S1) ${ }^{1}$ L-Trp-(Boc)-OMe (5m), ${ }^{3}$ L-Gln-(Trt)$\mathrm{OMe} \bullet \mathrm{HCl}(\mathbf{5 l}),{ }^{4} \mathrm{~L}-\beta$-Phe-OMe$\bullet \mathrm{HCl}(\mathbf{5 n}),{ }^{5} 4-n$-butylphenyl trifluoromethanesulfonate $(\mathbf{7 a}),{ }^{6} \mathrm{~m}$ tolyl trifluoromethanesulfonate $(\mathbf{7 b}),{ }^{7}$ 2-methoxy-4-propylphenyl trifluoromethanesulfonate $(\mathbf{7 c}){ }^{8}$ 4-acetamidophenyl trifluoromethanesulfonate (7e), ${ }^{9}$ methyl 3-trifluoromethanesulfonyloxybenzoate $\quad(\mathbf{7 g}){ }^{10} \quad$ 4-chlorophenyl trifluoromethanesulfonate $(\mathbf{7 h}),{ }^{11} \quad 3$-acetylphenyl trifluoromethanesulfonate $(\mathbf{7 j}),{ }^{12}$ 4-(trifluoromethyl)phenyl trifluoromethanesulfonate $(\mathbf{7 k}),{ }^{9}{ }^{3}$ quinolinyl trifluoromethanesulfonate (7l) ${ }^{13}$ estrone trifluoromethanesulfonate $(\mathbf{7 m}),{ }^{14}$ and 1 bromo-4-(methoxymethyl)benzene ${ }^{15}$ were prepared according to published procedures.

C) Instrumentation: Proton nuclear magnetic resonance spectra $\left({ }^{1} \mathrm{H}\right.$ NMR) were recorded at 400 or $500 \mathrm{MHz}$ at $24^{\circ} \mathrm{C}$, unless otherwise noted. Chemical shifts are expressed in parts per million ( $\mathrm{ppm}, \delta$ scale) downfield from tetramethylsilane and are referenced to residual protium in the NMR solvent $\left(\mathrm{CHCl}_{3}, \delta\right.$ 7.26; $\left.\mathrm{CHD}_{2} \mathrm{OD}, \delta 3.31\right)$. Data are represented as follows: chemical shift, multiplicity $(\mathrm{s}=$ singlet, $\mathrm{d}=$ doublet, $\mathrm{t}=$ triplet, $\mathrm{q}=$ quartet, $\mathrm{m}=$ multiplet and/or multiple resonances, $\mathrm{br}=$ broad, app $=$ apparent), coupling constant in Hertz, and integration. Protondecoupled carbon nuclear magnetic resonance spectra $\left({ }^{13} \mathrm{C}\right.$ NMR) were recorded at 100 or 125 $\mathrm{MHz}$ at $24{ }^{\circ} \mathrm{C}$, unless otherwise noted. Chemical shifts are expressed in parts per million (ppm, $\delta$ scale) downfield from tetramethylsilane and are referenced to the carbon resonances of the solvent $\left(\mathrm{CDCl}_{3}, \delta 77.2 ; \mathrm{CD}_{3} \mathrm{OD}, \delta 49.0\right)$. Proton-decoupled fluorine nuclear magnetic resonance spectra $\left({ }^{19} \mathrm{~F}\right.$ NMR) were recorded at $375 \mathrm{MHz}$ at $24{ }^{\circ} \mathrm{C}$, unless otherwise noted. Chemical shifts are expressed in parts per million ( $\mathrm{ppm}, \delta$ scale) downfield from fluorotrichloromethane $(0.00$ ppm). Proton-decoupled phosphorous nuclear magnetic resonance spectra $\left({ }^{31} \mathrm{P}\right.$ NMR) were recorded at $162 \mathrm{MHz}$ at $24{ }^{\circ} \mathrm{C}$, unless otherwise noted. Chemical shifts are expressed in parts per million (ppm, $\delta$ scale) downfield from $85 \%$ aq. phosphoric acid $(0.00 \mathrm{ppm})$. Attenuated total reflectance Fourier transform infrared spectra (ATR-FTIR) were obtained using a Thermo 
Scientific iD5 ATR Nicolet iS5 FT-IR spectrometer referenced to a polystyrene standard and data are reported as frequency of absorption $\left(\mathrm{cm}^{-1}\right)$. Melting points were obtained on a Mel-Temp capillary melting point apparatus. Elemental analyses were carried out by Atlantic Microlab, Inc., Norcross, GA. High Resolution Mass Spectra were obtained on a Bruker Daltonics APEXIV 4.7 Tesla Fourier transform ion cyclotron resonance mass spectrometer (FT-ICR-MS). High-pressure liquid chromatography (HPLC) was performed on Agilent 1200 Series chromatographs using chiral columns $(25 \mathrm{~cm})$ as noted for each compound. (Note: In some instances DL material was prepared by mixing a 1:1 ratio of the L-and D-amino acid ester. As this was often done on a small scale, the ratios of the enantiomeric products shown below may differ from 1:1). Optical rotations were measured on a Jasco P-1010 polarimeter equipped with a sodium (589 nm, D)

lamp. Optical rotation data are represented as follows: specific rotation $\left([\alpha]_{D}{ }^{T}\right)$, concentration $(\mathrm{g} / 100 \mathrm{~mL})$, and solvent. 


\section{Experimental Procedures and Characterization Data ${ }^{16}$}

A) $N$-Arylation of Amino Acid Esters
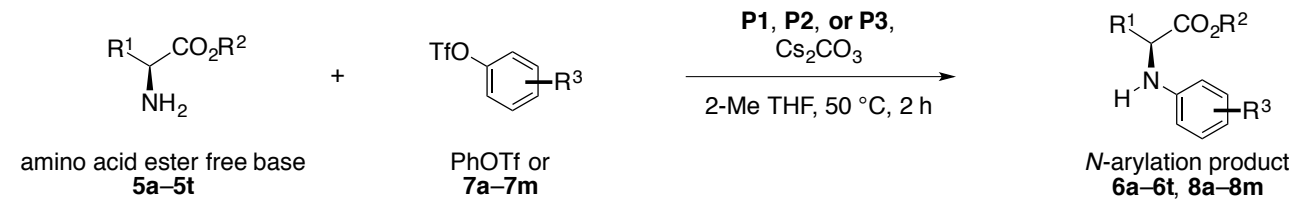

General Procedure: A $25 \mathrm{~mL}$ screw-cap tube equipped with a stir bar and Teflon septum was charged sequentially with amino acid ester, if solid, (1.00 mmol, 1.00 equiv), triflate, if solid, (1.00 mmol, 1.00 equiv), P1 (43.0 mg, $50.0 \mu \mathrm{mol}, 5.0 \mathrm{~mol} \%$ ) or P3 (43.0 mg, $50.0 \mu \mathrm{mol}, 5.0$ $\mathrm{mol} \%$ ), and cesium carbonate $(977 \mathrm{mg}, 3.00 \mathrm{mmol}, 3.00$ equiv). The reaction test tube was capped and then evacuated and backfilled with argon by piercing with a needle attached to a Schlenk line (this process was repeated a total of three times). Amino acid ester and/or triflate, if liquid, and 2-methyltetrahydrofuran $(2.00 \mathrm{~mL})$ were added sequentially to the reaction test tube. The reaction test tube was placed in an oil bath that had been preheated to $50{ }^{\circ} \mathrm{C}$. The reaction mixture was stirred and heated at $50{ }^{\circ} \mathrm{C}$ for $2 \mathrm{~h}$. The reaction mixture was allowed to cool over $20 \mathrm{~min}$ to $\mathrm{rt}$. The cooled product mixture was diluted with $\mathrm{CH}_{2} \mathrm{Cl}_{2}(5.00 \mathrm{~mL})$. The diluted product mixture was filtered through Celite and concentrated to dryness. The residue obtained was purified by automated flash-column chromatography. The yields reported are the average of two experiments. The enantiomeric excesses (\% ee) were determined by HPLC analysis using chiral stationary phases.<smiles>CCCCOC(=O)C(Cc1ccccc1)Nc1ccccc1</smiles>

6a

Following the general procedure, a mixture of L-Phe-Ot-Bu (5a, $221 \mathrm{mg}, 1.00 \mathrm{mmol}, 1.00$ equiv), phenyl trifluoromethanesulfonate $(162 \mu \mathrm{L}, 1.00 \mathrm{mmol}, 1$ equiv), P1 (43.0 mg, $50.0 \mu \mathrm{mol}, 5.0$ mol\%), cesium carbonate ( $977 \mathrm{mg}, 3.00 \mathrm{mmol}, 3.00$ equiv), and 2-methyltetrahydrofuran (2.00 $\mathrm{mL}$ ) was stirred at $50{ }^{\circ} \mathrm{C}$ for $2 \mathrm{~h}$. The crude product was purified by automated flash-column chromatography (eluting with $2 \%$ acetone-hexanes initially, grading to $20 \%$ acetone-hexanes, linear gradient) to afford 6a as a white solid. Yield: $268 \mathrm{mg}, 90 \% . \mathrm{mp}=83-85{ }^{\circ} \mathrm{C} .{ }^{1} \mathrm{H}$ NMR $(400$ $\left.\mathrm{MHz}, \mathrm{CDCl}_{3}\right) \delta 7.37-7.25(\mathrm{~m}, 5 \mathrm{H}), 7.22(\mathrm{dd}, J=8.5,7.4 \mathrm{~Hz}, 2 \mathrm{H}), 6.79(\mathrm{t}, J=7.3 \mathrm{~Hz}, 1 \mathrm{H}), 6.68$ $(\mathrm{d}, J=7.7 \mathrm{~Hz}, 2 \mathrm{H}), 4.31(\mathrm{t}, J=6.4 \mathrm{~Hz}, 1 \mathrm{H}), 3.16(\mathrm{~d}, J=6.4 \mathrm{~Hz}, 2 \mathrm{H}), 1.40(\mathrm{~s}, 9 \mathrm{H}) .{ }^{13} \mathrm{C}$ NMR $(100$ $\left.\mathrm{MHz}, \mathrm{CDCl}_{3}\right) \delta 172.3,146.7,136.8,129.6,129.4,128.4,126.9,118.3,113.7,81.8,58.3,38.7$, 28.0. IR (neat, $\mathrm{cm}^{-1}$ ): $3358,2976,1704,1601,1157,693$. Anal. Calcd. for $\mathrm{C}_{19} \mathrm{H}_{23} \mathrm{NO}_{2}: \mathrm{C}, 76.74$; $\mathrm{H}, 7.80$, Found: C, 76.86; H, 7.87. $[\alpha]_{\mathrm{D}}{ }^{24}+18.6\left(c 1.0, \mathrm{CHCl}_{3}\right)$. HPLC analysis $(\mathrm{OJ}-\mathrm{H}, 2 \% \mathrm{IPA}-$ hexanes, $0.8 \mathrm{~mL} / \mathrm{min}, 254 \mathrm{~nm}$ ) indicated 94\% ee: $\mathrm{tR}$ (minor) $=14.4 \mathrm{~min}$, tR (major) $=19.0 \mathrm{~min}$.

$\int_{\mathrm{NHPh}}^{\mathrm{CO}_{2} t-\mathrm{Bu}}$

6b

Following the general procedure, a mixture of Gly-Ot-Bu (5b, $131 \mathrm{mg}, 1.00 \mathrm{mmol}, 1.00$ equiv), phenyl trifluoromethanesulfonate $(162 \mu \mathrm{L}, 1.00 \mathrm{mmol}, 1$ equiv), P1 (43.0 mg, $50.0 \mu \mathrm{mol}, 5.0$ $\mathrm{mol} \%$ ), cesium carbonate ( $977 \mathrm{mg}, 3.00 \mathrm{mmol}, 3.00$ equiv), and 2-methyltetrahydrofuran (2.00 $\mathrm{mL}$ ) was stirred at $50{ }^{\circ} \mathrm{C}$ for $2 \mathrm{~h}$. The crude product was purified by automated flash-column chromatography (eluting with $2 \%$ acetone-hexanes initially, grading to $20 \%$ acetone-hexanes, linear gradient) to afford $\mathbf{6 b}$ as a clear oil. Yield: $200 \mathrm{mg}, 97 \% .{ }^{1} \mathrm{H} \mathrm{NMR}\left(400 \mathrm{MHz}, \mathrm{CDCl}_{3}\right) \delta$ $7.30-7.22(\mathrm{~m}, 2 \mathrm{H}), 6.82(\mathrm{t}, J=7.3 \mathrm{~Hz}, 1 \mathrm{H}), 6.67(\mathrm{~d}, J=7.8 \mathrm{~Hz}, 2 \mathrm{H}), 4.38(\mathrm{~s}, 1 \mathrm{H}), 3.85(\mathrm{~s}, 2 \mathrm{H})$, 
1.57 (s, 9H). ${ }^{13} \mathrm{C}$ NMR $\left(101 \mathrm{MHz}, \mathrm{CDCl}_{3}\right) \delta 170.2,147.2,129.2,117.9,112.9,81.8,46.5,28.0$. IR (neat, $\mathrm{cm}^{-1}$ ): $3401,2977,1732,1604,1508,1150$. Anal. Calcd. for $\mathrm{C}_{12} \mathrm{H}_{17} \mathrm{NO}_{2}: \mathrm{C}, 69.54 ; \mathrm{H}$, 8.27, Found: C, 69.26; H, 8.25.

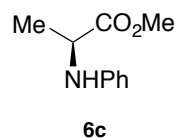

Following the general procedure, a mixture of L-Ala-OMe (5c, $103 \mathrm{mg}, 1.00 \mathrm{mmol}, 1.00$ equiv), phenyl trifluoromethanesulfonate $(162 \mu \mathrm{L}, 1.00 \mathrm{mmol}, 1$ equiv), P1 (43.0 mg, $50.0 \mu \mathrm{mol}, 5.0$ mol\%), cesium carbonate ( $977 \mathrm{mg}, 3.00 \mathrm{mmol}, 3.00$ equiv), and 2-methyltetrahydrofuran (2.00 $\mathrm{mL}$ ) was stirred at $50{ }^{\circ} \mathrm{C}$ for $2 \mathrm{~h}$. The crude product was purified by automated flash-column chromatography with a new column (eluting with $1 \%$ acetone-pentane initially, grading to $10 \%$ acetone-pentane, linear gradient) to afford $\mathbf{6 c}$ as a yellow oil. Yield: $157 \mathrm{mg}, 88 \% .{ }^{1} \mathrm{H}$ NMR $\left(400 \mathrm{MHz}, \mathrm{CDCl}_{3}\right) \delta 7.21(\mathrm{dd}, J=8.5,7.4 \mathrm{~Hz}, 2 \mathrm{H}), 6.77(\mathrm{t}, J=7.3 \mathrm{~Hz}, 1 \mathrm{H}), 6.64(\mathrm{~d}, J=7.7 \mathrm{~Hz}$, 2H), 4.25 (br s, $1 \mathrm{H}), 4.19$ (q, $J=7.0 \mathrm{~Hz}, 1 \mathrm{H}), 3.75(\mathrm{~s}, 3 \mathrm{H}), 1.50(\mathrm{~d}, J=6.9 \mathrm{~Hz}, 3 \mathrm{H}) .{ }^{13} \mathrm{C}$ NMR $\left(100 \mathrm{MHz}, \mathrm{CDCl}_{3}\right) \delta 175.1,146.6,129.3,118.3,113.3,52.2,51.9,18.9$. IR (neat, $\left.\mathrm{cm}^{-1}\right): 3394$, 2951, 1732, 1602, 1506, 1156, 748, 692. Anal. Calcd. for $\mathrm{C}_{10} \mathrm{H}_{13} \mathrm{NO}_{2}$ : C, 67.02; H, 7.31, Found: $\mathrm{C}, 67.14 ; \mathrm{H}, 7.15 .[\alpha]_{\mathrm{D}}{ }^{24}-53.6\left(c 1.0, \mathrm{CHCl}_{3}\right)$. HPLC analysis $(\mathrm{OJ}-\mathrm{H}, 10 \%$ IPA-hexanes, 1.0 $\mathrm{mL} / \mathrm{min}, 254 \mathrm{~nm}$ ) indicated 95\% ee: $\mathrm{tR}($ minor $)=16.2 \mathrm{~min}$, $\mathrm{tR}($ major $)=24.2 \mathrm{~min}$.

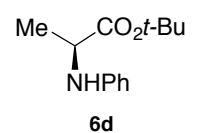

Following the general procedure, a mixture of L-Ala-Ot-Bu (5d, $145 \mathrm{mg}, 1.00 \mathrm{mmol}, 1.00$ equiv), phenyl trifluoromethanesulfonate $(162 \mu \mathrm{L}, 1.00 \mathrm{mmol}, 1$ equiv), P1 (43.0 mg, $50.0 \mu \mathrm{mol}, 5.0$ mol\%), cesium carbonate ( $977 \mathrm{mg}, 3.00 \mathrm{mmol}, 3.00$ equiv), and 2-methyltetrahydrofuran (2.00 $\mathrm{mL}$ ) was stirred at $50{ }^{\circ} \mathrm{C}$ for $2 \mathrm{~h}$. The crude product was purified by automated flash-column chromatography (eluting with $1 \%$ acetone-pentane initially, grading to $10 \%$ acetone-pentane, linear gradient) to afford $\mathbf{6 d}$ as a clear oil. Yield: $198 \mathrm{mg}, 90 \% .{ }^{1} \mathrm{H}$ NMR $\left(400 \mathrm{MHz}, \mathrm{CDCl}_{3}\right) \delta$ $7.09-7.03(\mathrm{~m}, 2 \mathrm{H}), 6.62(\mathrm{t}, J=7.3 \mathrm{~Hz}, 1 \mathrm{H}), 6.51(\mathrm{~d}, J=7.7 \mathrm{~Hz}, 2 \mathrm{H}), 4.16(\mathrm{br} \mathrm{s}, 1 \mathrm{H}), 3.92(\mathrm{q}, J=$ $6.9 \mathrm{~Hz}, 1 \mathrm{H}), 1.33(\mathrm{~s}, 9 \mathrm{H}), 1.32(\mathrm{~s}, 3 \mathrm{H}) .{ }^{13} \mathrm{C} \mathrm{NMR}\left(100 \mathrm{MHz}, \mathrm{CDCl}_{3}\right) \delta 173.8,146.8,129.2,118.1$, 113.5, 81.4, 52.6, 28.0, 18.8. IR (neat, $\mathrm{cm}^{-1}$ ): 3395, 2977, 1726, 1603, 1505, 1145, 746, 691. Anal. Calcd. for $\mathrm{C}_{13} \mathrm{H}_{19} \mathrm{NO}_{2}$ : C, 70.56; H, 8.65, Found: C, 70.70; H, 8.47. $[\alpha]_{\mathrm{D}}{ }^{24}-38.6\left(c 1.0, \mathrm{CHCl}_{3}\right)$. HPLC analysis (OJ-H, 5\% IPA-hexanes, $0.8 \mathrm{~mL} / \mathrm{min}, 254 \mathrm{~nm}$ ) indicated $96 \%$ ee: tR (minor) $=$ $8.7 \mathrm{~min}, \mathrm{tR}$ (major) $=9.3 \mathrm{~min}$.

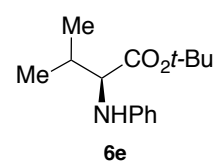

Following the general procedure, a mixture of L-Val-Ot-Bu (5e, $173 \mathrm{mg}, 1.00 \mathrm{mmol}, 1.00$ equiv), phenyl trifluoromethanesulfonate $(162 \mu \mathrm{L}, 1.00 \mathrm{mmol}, 1$ equiv), P1 (43.0 mg, $50.0 \mu \mathrm{mol}, 5.0$ mol\%), cesium carbonate ( $977 \mathrm{mg}, 3.00 \mathrm{mmol}, 3.00$ equiv), and 2-methyltetrahydrofuran $(2.00$ $\mathrm{mL}$ ) was stirred at $50{ }^{\circ} \mathrm{C}$ for $14 \mathrm{~h}$. The crude product was purified by automated flash-column chromatography (eluting with $1 \%$ EtOAc-hexanes initially, grading to $10 \%$ EtOAc-hexanes, linear gradient) to afford $6 \mathbf{e}$ as a white solid. Yield: $208 \mathrm{mg}, 83 \% . \mathrm{mp}=82-84{ }^{\circ} \mathrm{C}$. ${ }^{1} \mathrm{H}$ NMR $(400$ $\left.\mathrm{MHz}, \mathrm{CDCl}_{3}\right) \delta 7.21-7.14(\mathrm{~m}, 2 \mathrm{H}), 6.73(\mathrm{t}, J=7.3 \mathrm{~Hz}, 1 \mathrm{H}), 6.65(\mathrm{~d}, J=7.7 \mathrm{~Hz}, 2 \mathrm{H}), 4.22$ (br s, $1 \mathrm{H}), 3.77(\mathrm{~d}, J=5.6 \mathrm{~Hz}, 1 \mathrm{H}), 2.13(\mathrm{dp}, J=13.5,6.8 \mathrm{~Hz}, 1 \mathrm{H}), 1.44(\mathrm{~s}, 9 \mathrm{H}), 1.05(\mathrm{dd}, J=8.9,6.8$ $\mathrm{Hz}, 6 \mathrm{H}) .{ }^{13} \mathrm{C}$ NMR $\left(100 \mathrm{MHz}, \mathrm{CDCl}_{3}\right) \delta 172.8,147.7,129.3,118.1,113.8,81.6,63.0,31.6,28.2$, 19.1, 18.8. IR (neat, $\mathrm{cm}^{-1}$ ): 3382, 2970, 1707, 1604, 1257, 1156, 745, 691. Anal. Calcd. for $\mathrm{C}_{15} \mathrm{H}_{23} \mathrm{NO}_{2}$ : C, 72.25; H, 9.30, Found: C, 72.35; H, 9.44. $[\alpha]_{\mathrm{D}}{ }^{24}-70.1\left(c 1.0, \mathrm{CHCl}_{3}\right)$. HPLC 
analysis (OJ-H, $0.5 \%$ IPA-hexanes, $0.5 \mathrm{~mL} / \mathrm{min}, 254 \mathrm{~nm}$ ) indicated $87 \%$ ee: tR (major) $=12.3$ min, $\mathrm{tR}($ minor $)=13.3 \mathrm{~min}$.<smiles>CC(C)C(Nc1ccccc1)C(=O)OCc1ccccc1</smiles>

Following the general procedure, a mixture of L-Val-OBn (5f, $207 \mathrm{mg}, 1.00 \mathrm{mmol}, 1.00$ equiv), phenyl trifluoromethanesulfonate $(162 \mu \mathrm{L}, 1.00 \mathrm{mmol}, 1$ equiv), P1 (43.0 mg, $50.0 \mu \mathrm{mol}, 5.0$ mol\%), cesium carbonate $(977 \mathrm{mg}, 3.00 \mathrm{mmol}, 3.00$ equiv), and 2-methyltetrahydrofuran $(2.00$ $\mathrm{mL}$ ) was stirred at $50{ }^{\circ} \mathrm{C}$ for $14 \mathrm{~h}$. The crude product was purified by automated flash-column chromatography (eluting with $2 \%$ ether-pentane initially, grading to $20 \%$ ether-pentane, linear gradient) to afford $\mathbf{6 f}$ as a clear oil. Yield: $216 \mathrm{mg}, 76 \%$. ${ }^{1} \mathrm{H}$ NMR $\left(400 \mathrm{MHz}, \mathrm{CDCl}_{3}\right) \delta 7.48-$ $7.36(\mathrm{~m}, 5 \mathrm{H}), 7.27(\mathrm{t}, J=7.9 \mathrm{~Hz}, 2 \mathrm{H}), 6.85(\mathrm{t}, J=7.3 \mathrm{~Hz}, 1 \mathrm{H}), 6.75(\mathrm{~d}, J=7.8 \mathrm{~Hz}, 2 \mathrm{H}), 5.25$ (app d, $J=2.9 \mathrm{~Hz}, 2 \mathrm{H}), 4.27(\mathrm{br} \mathrm{d}, J=7.8 \mathrm{~Hz}, 1 \mathrm{H}), 4.04(\mathrm{t}, J=7.1 \mathrm{~Hz}, 1 \mathrm{H}), 2.31-2.18(\mathrm{~m}, J=6.7 \mathrm{~Hz}$, $1 \mathrm{H}), 1.13(\mathrm{dd}, J=10.2,6.8 \mathrm{~Hz}, 6 \mathrm{H}) .{ }^{13} \mathrm{C}$ NMR $\left(100 \mathrm{MHz}, \mathrm{CDCl}_{3}\right) \delta 173.6,147.4,135.6,129.3$, 128.6, 128.4, 128.3, 118.3, 113.7, 66.7, 62.6, 31.6, 19.2, 18.7. IR (neat, $\mathrm{cm}^{-1}$ ): 3384, 2961, 1729, 1601, 1145, 746, 691. Anal. Calcd. for $\mathrm{C}_{18} \mathrm{H}_{21} \mathrm{NO}_{2}: \mathrm{C}, 76.30 ; \mathrm{H}, 7.47$, Found: C, 76.34; H, 7.39. $[\alpha]_{\mathrm{D}}{ }^{24}-40.6\left(c 1.0, \mathrm{CHCl}_{3}\right)$. HPLC analysis (OJ-H, $10 \%$ IPA-hexanes, $\left.1.0 \mathrm{~mL} / \mathrm{min}, 254 \mathrm{~nm}\right)$ indicated $94 \%$ ee: $\mathrm{tR}$ (minor) $=19.0 \mathrm{~min}$, $\mathrm{tR}$ (major) $=24.0 \mathrm{~min}$.

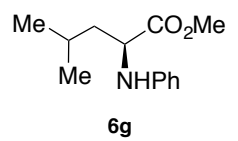

Following the general procedure, a mixture of L-Leu-OMe (5g, $145 \mathrm{mg}, 1.00 \mathrm{mmol}, 1.00$ equiv), phenyl trifluoromethanesulfonate $(162 \mu \mathrm{L}, 1.00 \mathrm{mmol}, 1$ equiv), P1 (43.0 mg, $50.0 \mu \mathrm{mol}, 5.0$ mol\%), cesium carbonate ( $977 \mathrm{mg}, 3.00 \mathrm{mmol}, 3.00$ equiv), and 2-methyltetrahydrofuran (2.00 $\mathrm{mL}$ ) was stirred at $50{ }^{\circ} \mathrm{C}$ for $2 \mathrm{~h}$. The crude product was purified by automated flash-column chromatography (eluting with $1 \%$ acetone-pentane initially, grading to $10 \%$ acetone-pentane, linear gradient) to afford $\mathbf{6 g}$ as a white solid. Yield: $198 \mathrm{mg}, 90 \% . \mathrm{mp}=49-50{ }^{\circ} \mathrm{C}$. ${ }^{1} \mathrm{H}$ NMR $(400$ $\left.\mathrm{MHz}, \mathrm{CDCl}_{3}\right) \delta 7.20(\mathrm{dd}, J=8.6,7.3 \mathrm{~Hz}, 2 \mathrm{H}), 6.77(\mathrm{t}, J=7.3 \mathrm{~Hz}, 1 \mathrm{H}), 6.65(\mathrm{~d}, J=7.8 \mathrm{~Hz}, 2 \mathrm{H})$, $4.17-4.11(\mathrm{~m}, 1 \mathrm{H}), 4.08$ (br s, 1H), $3.72(\mathrm{~s}, 3 \mathrm{H}), 1.84$ (hept, $J=6.3 \mathrm{~Hz}, 1 \mathrm{H}), 1.69$ (td, $J=7.0,2.7$ $\mathrm{Hz}, 2 \mathrm{H}), 1.01(\mathrm{dd}, J=18.1,6.6 \mathrm{~Hz}, 6 \mathrm{H}) .{ }^{13} \mathrm{C} \mathrm{NMR}\left(100 \mathrm{MHz}, \mathrm{CDCl}_{3}\right) \delta 175.2,147.0,129.4$, 118.4, 113.5, 55.2, 52.0, 42.4, 24.9, 22.8, 22.3. IR (neat, $\mathrm{cm}^{-1}$ ): 3383, 2954, 1718, 1602, 745, 689 . Anal. Calcd. for $\mathrm{C}_{13} \mathrm{H}_{19} \mathrm{NO}_{2}$ : C, 70.56; H, 8.65, Found: C, 70.68; H, 8.67. [ $\left.\alpha\right]_{\mathrm{D}}{ }^{24}-70.7$ (c 1.0, $\left.\mathrm{CHCl}_{3}\right)$. HPLC analysis (OJ-H, $5 \%$ IPA-hexanes, $\left.0.8 \mathrm{~mL} / \mathrm{min}, 254 \mathrm{~nm}\right)$ indicated $96 \%$ ee: tR $($ minor $)=10.2 \mathrm{~min}, \mathrm{tR}($ major $)=15.0 \mathrm{~min}$.

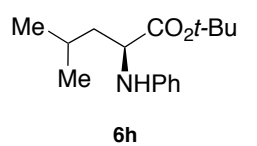

Following the general procedure, a mixture of L-Leu-Ot-Bu (5h, $187 \mathrm{mg}, 1.00 \mathrm{mmol}, 1.00$ equiv), phenyl trifluoromethanesulfonate $(162 \mu \mathrm{L}, 1.00 \mathrm{mmol}, 1$ equiv), P1 (43.0 mg, $50.0 \mu \mathrm{mol}, 5.0$ $\mathrm{mol} \%$ ), cesium carbonate ( $977 \mathrm{mg}, 3.00 \mathrm{mmol}, 3.00$ equiv), and 2-methyltetrahydrofuran (2.00 $\mathrm{mL}$ ) was stirred at $50{ }^{\circ} \mathrm{C}$ for $2 \mathrm{~h}$. The crude product was purified by automated flash-column chromatography (eluting with $2 \%$ EtOAc-hexanes initially, grading to $10 \%$ EtOAc-hexanes, linear gradient) to afford $\mathbf{6 h}$ as a white solid. Yield: $231 \mathrm{mg}, 88 \% . \mathrm{mp}=68-70{ }^{\circ} \mathrm{C} .{ }^{1} \mathrm{H}$ NMR $\left(400 \mathrm{MHz}, \mathrm{CDCl}_{3}\right) \delta 7.23-7.15(\mathrm{~m}, 2 \mathrm{H}), 6.75(\mathrm{t}, J=7.3 \mathrm{~Hz}, 1 \mathrm{H}), 6.66(\mathrm{~d}, J=7.7 \mathrm{~Hz}, 2 \mathrm{H}), 4.06$ (br s, 1H), 4.03-3.98 (m, 1H), $1.87(\mathrm{dp}, J=13.4,6.7 \mathrm{~Hz}, 1 \mathrm{H}), 1.67(\mathrm{tt}, J=13.9,6.5 \mathrm{~Hz}, 2 \mathrm{H}), 1.45$ (s, 9H), $1.02(\mathrm{dd}, J=20.8,6.6 \mathrm{~Hz}, 6 \mathrm{H}) .{ }^{13} \mathrm{C} \mathrm{NMR}\left(100 \mathrm{MHz}, \mathrm{CDCl}_{3}\right) \delta 174.0,147.3,129.2,118.1$, 113.6, 81.3, 55.9, 42.4, 28.0, 25.0, 22.8, 22.5. IR (neat, $\mathrm{cm}^{-1}$ ): 3378, 2955, 1703, 1604, 1305, 
1149, 761, 693. Anal. Calcd. for $\mathrm{C}_{16} \mathrm{H}_{25} \mathrm{NO}_{2}$ : C, 72.97; H, 9.57, Found: C, 73.16; H, 9.69. [ $\left.\alpha\right]_{\mathrm{D}}{ }^{24}$ 70.1 ( c 1.0, $\left.\mathrm{CHCl}_{3}\right)$. HPLC analysis $(\mathrm{OJ}-\mathrm{H}, 1 \%$ IPA-hexanes, $0.8 \mathrm{~mL} / \mathrm{min}, 254 \mathrm{~nm})$ indicated $97 \%$ ee: $\mathrm{tR}($ minor $)=6.3 \mathrm{~min}, \mathrm{tR}$ (major) $=7.0 \mathrm{~min}$.

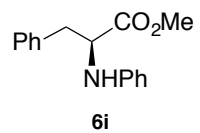

Following the general procedure, a mixture of L-Phe-OMe (5i, $179 \mathrm{mg}, 1.00 \mathrm{mmol}, 1.00$ equiv), phenyl trifluoromethanesulfonate $(162 \mu \mathrm{L}, 1.00 \mathrm{mmol}, 1$ equiv), P1 (43.0 mg, $50.0 \mu \mathrm{mol}, 5.0$ mol\%), cesium carbonate ( $977 \mathrm{mg}, 3.00 \mathrm{mmol}, 3.00$ equiv), and 2-methyltetrahydrofuran (2.00 $\mathrm{mL}$ ) was stirred at $50{ }^{\circ} \mathrm{C}$ for $2 \mathrm{~h}$. The crude product was purified by automated flash-column chromatography with a new column (eluting with $2 \%$ acetone-hexanes initially, grading to $20 \%$ acetone-hexanes, linear gradient) to afford $\mathbf{6 i}$ as a yellow oil. Yield: $244 \mathrm{mg}, 96 \%$. ${ }^{1} \mathrm{H}$ NMR (400 $\left.\mathrm{MHz}, \mathrm{CDCl}_{3}\right) \delta 7.38(\mathrm{dt}, J=14.7,6.9 \mathrm{~Hz}, 3 \mathrm{H}), 7.32-7.25(\mathrm{~m}, 4 \mathrm{H}), 6.87(\mathrm{t}, J=7.3 \mathrm{~Hz}, 1 \mathrm{H}), 6.73$ $(\mathrm{d}, J=7.8 \mathrm{~Hz}, 2 \mathrm{H}), 4.50$ (t, $J=6.2 \mathrm{~Hz}, 1 \mathrm{H}), 4.39$ (br s, $1 \mathrm{H}), 3.75(\mathrm{~s}, 3 \mathrm{H}), 3.31-3.18(\mathrm{~m}, 2 \mathrm{H}) .{ }^{13} \mathrm{C}$ $\operatorname{NMR}\left(100 \mathrm{MHz}, \mathrm{CDCl}_{3}\right) \delta 173.5,146.3,136.3,129.3,129.2,128.5,127.0,118.4,113.6,57.8$, 52.0, 38.6. IR (neat, $\mathrm{cm}^{-1}$ ): 3353, 3026, 1731, 1601, 1496, 692. Anal. Calcd. for $\mathrm{C}_{16} \mathrm{H}_{17} \mathrm{NO}_{2}$ : $\mathrm{C}$, 75.72; H, 6.71, Found: C, 75.42; H, 6.80. $[\alpha]_{\mathrm{D}}{ }^{24}+38.2\left(c 1.0, \mathrm{CHCl}_{3}\right)$. HPLC analysis $(\mathrm{OJ}-\mathrm{H}, 10 \%$ IPA-hexanes, $1.0 \mathrm{~mL} / \mathrm{min}, 254 \mathrm{~nm}$ ) indicated $87 \%$ ee: tR (minor) $=30.7 \mathrm{~min}$, tR (major) $=42.8$ min.

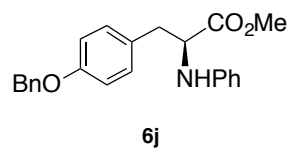

Following the general procedure, a mixture of L-Tyr-(Bn)-OMe (5j, $285 \mathrm{mg}, 1.00 \mathrm{mmol}, 1.00$ equiv), phenyl trifluoromethanesulfonate ( $162 \mu \mathrm{L}, 1.00 \mathrm{mmol}, 1$ equiv), P1 (43.0 mg, $50.0 \mu \mathrm{mol}$, $5.0 \mathrm{~mol} \%$ ), cesium carbonate ( $977 \mathrm{mg}, 3.00 \mathrm{mmol}, 3.00$ equiv), and 2-methyltetrahydrofuran $(2.00 \mathrm{~mL})$ was stirred at $50{ }^{\circ} \mathrm{C}$ for $2 \mathrm{~h}$. The crude product was purified by automated flashcolumn chromatography with a new column (eluting with $10 \%$ ether-pentane initially, grading to $20 \%$ ether-pentane, linear gradient) to afford $\mathbf{6 j}$ as a clear oil. Yield: $343 \mathrm{mg}, 95 \% .{ }^{1} \mathrm{H}$ NMR $\left(400 \mathrm{MHz}, \mathrm{CDCl}_{3}\right) \delta$ 7.55-7.39 (m, 5H), 7.32-7.25 (m, 2H), 7.21-7.15 (m, 2H), 7.03-6.99 (m, $2 \mathrm{H}), 6.85(\mathrm{tt}, J=7.4,1.0 \mathrm{~Hz}, 1 \mathrm{H}), 6.73-6.68(\mathrm{~m}, 2 \mathrm{H}), 5.12(\mathrm{~s}, 2 \mathrm{H}), 4.44(\mathrm{~s}, 1 \mathrm{H}), 4.29(\mathrm{~s}, 1 \mathrm{H})$, $3.75(\mathrm{~s}, 3 \mathrm{H}), 3.24-3.10(\mathrm{~m}, 2 \mathrm{H}) .{ }^{13} \mathrm{C}$ NMR $\left(100 \mathrm{MHz}, \mathrm{CDCl}_{3}\right) \delta 173.7,157.9,146.4,137.0,130.3$, 129.4, 128.6 (2C), 128.0, 127.5, 118.4, 114.9, 113.6, 70.0, 57.8, 52.1, 37.7. IR (neat, $\mathrm{cm}^{-1}$ ): 3397, 3030, 1736, 1602, 1506, 1239, 1175, 748, 693. Anal. Calcd. for $\mathrm{C}_{23} \mathrm{H}_{23} \mathrm{NO}_{3}: \mathrm{C}, 76.43 ; \mathrm{H}, 6.41$, Found: C, 76.23; H, 6.44. $[\alpha]_{\mathrm{D}}{ }^{24}+47.6\left(c 1.0, \mathrm{CHCl}_{3}\right)$. HPLC analysis (OD-H, 10\% IPA-hexanes, $1.0 \mathrm{~mL} / \mathrm{min}, 254 \mathrm{~nm}$ ) indicated $96 \%$ ee: $\mathrm{tR}$ (major) $=29.8 \mathrm{~min}, \mathrm{tR}$ (minor) $=54.4 \mathrm{~min}$.

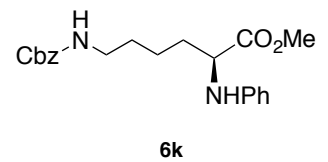

Following the general procedure, a mixture of L-Lys-(Cbz)-OMe (5k $, 294 \mathrm{mg}, 1.00 \mathrm{mmol}, 1.00$ equiv), phenyl trifluoromethanesulfonate $(162 \mu \mathrm{L}, 1.00 \mathrm{mmol}, 1$ equiv), P1 (43.0 mg, $50.0 \mu \mathrm{mol}$, $5.0 \mathrm{~mol} \%$ ), cesium carbonate ( $977 \mathrm{mg}, 3.00 \mathrm{mmol}, 3.00$ equiv), and 2-methyltetrahydrofuran $(2.00 \mathrm{~mL})$ was stirred at $50{ }^{\circ} \mathrm{C}$ for $2 \mathrm{~h}$. The crude product was purified by automated flashcolumn chromatography (eluting with $8 \%$ EtOAc-hexanes initially, grading to 66\% EtOAchexanes, linear gradient) to afford $\mathbf{6 k}$ as a yellow oil. Yield: $340 \mathrm{mg}, 92 \%$. ${ }^{1} \mathrm{H}$ NMR $(400 \mathrm{MHz}$, $\left.\mathrm{CDCl}_{3}\right) \delta 7.38-7.29(\mathrm{~m}, 5 \mathrm{H}), 7.21-7.15(\mathrm{~m}, 2 \mathrm{H}), 6.75(\mathrm{tt}, J=7.3,1.0 \mathrm{~Hz}, 1 \mathrm{H}), 6.64-6.59(\mathrm{~m}, 2 \mathrm{H})$, $5.11(\mathrm{~s}, 2 \mathrm{H}), 4.94(\mathrm{~s}, 1 \mathrm{H}), 4.21(\mathrm{~s}, 1 \mathrm{H}), 4.06(\mathrm{~s}, 1 \mathrm{H}), 3.71(\mathrm{~s}, 3 \mathrm{H}), 3.19(\mathrm{q}, J=6.4 \mathrm{~Hz}, 2 \mathrm{H}), 1.93-$ $1.68(\mathrm{~m}, 2 \mathrm{H}), 1.59-1.38(\mathrm{~m}, 4 \mathrm{H}) .{ }^{13} \mathrm{C}$ NMR $\left(100 \mathrm{MHz}, \mathrm{CDCl}_{3}\right) \delta 174.6,156.5,146.8,136.6$, 
129.4, 128.5, 128.1 (2 C), 118.3, 113.4, 66.6, 56.4, 52.2, 40.7, 32.6, 29.7, 22.8. IR (neat, $\mathrm{cm}^{-1}$ ): 3357, 2949, 1702, 1603, 1506, 1242, 693. Anal. Calcd. for $\mathrm{C}_{21} \mathrm{H}_{26} \mathrm{~N}_{2} \mathrm{O}_{4}$ : C, 68.09; H, 7.07, Found: C, 68.25; H, 6.99. $[\alpha]_{\mathrm{D}}{ }^{24}-20.3\left(c\right.$ 1.0, $\left.\mathrm{CHCl}_{3}\right)$. HPLC analysis (OD-H, 20\% IPA-hexanes, 1.0 $\mathrm{mL} / \mathrm{min}, 254 \mathrm{~nm}$ ) indicated 94\% ee: $\mathrm{tR}$ (major) $=19.7 \mathrm{~min}, \mathrm{tR}($ minor $)=40.3 \mathrm{~min}$.

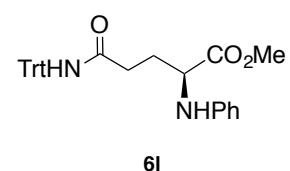

Following the general procedure, a mixture of L-Gln-(Trt)-OMe (5l, $402 \mathrm{mg}, 1.00 \mathrm{mmol}, 1.00$ equiv), phenyl trifluoromethanesulfonate (162 $\mu \mathrm{L}, 1.00 \mathrm{mmol}, 1$ equiv), P1 (43.0 mg, $50.0 \mu \mathrm{mol}$, $5.0 \mathrm{~mol} \%$ ), cesium carbonate ( $977 \mathrm{mg}, 3.00 \mathrm{mmol}, 3.00$ equiv), and 2-methyltetrahydrofuran $(2.00 \mathrm{~mL})$ was stirred at $50{ }^{\circ} \mathrm{C}$ for $2 \mathrm{~h}$. The crude product was purified by automated flashcolumn chromatography (eluting with $1 \%$ acetone $-\mathrm{CH}_{2} \mathrm{Cl}_{2}$ initially, grading to $10 \%$ acetone$\mathrm{CH}_{2} \mathrm{Cl}_{2}$, linear gradient) to afford $6 \mathbf{l}$ as a white solid. Yield: $420 \mathrm{mg}, 88 \% . \mathrm{mp}=187-190{ }^{\circ} \mathrm{C} .{ }^{1} \mathrm{H}$ NMR (400 MHz, $\left.\mathrm{CDCl}_{3}\right) \delta$ 7.34-7.24 (m, 9H), 7.24-7.15 (m, 8H), $6.78(\mathrm{t}, J=7.3 \mathrm{~Hz}, 1 \mathrm{H}), 6.66$ $(\mathrm{s}, 1 \mathrm{H}), 6.60(\mathrm{~d}, J=7.7 \mathrm{~Hz}, 2 \mathrm{H}), 4.43($ br s, $1 \mathrm{H}), 4.13$ (dd, $J=8.4,5.3 \mathrm{~Hz}, 1 \mathrm{H}), 3.70(\mathrm{~s}, 3 \mathrm{H})$, 2.60-2.39 (m, 2H), 2.26-2.05 (m, 2H). ${ }^{13} \mathrm{C}$ NMR (100 MHz, $\left.\mathrm{CDCl}_{3}\right) \delta 174.4,170.8,146.9,144.8$, 129.5, 128.8, 128.1, 127.2, 118.7, 113.8, 70.8, 56.5, 52.4, 33.6, 28.0. IR (neat, $\mathrm{cm}^{-1}$ ): 3271, 1741, 1647, 1536, 748, 697. Anal. Calcd. for $\mathrm{C}_{31} \mathrm{H}_{30} \mathrm{~N}_{2} \mathrm{O}_{3}$ : C, 77.80; H, 6.32, Found: C, 77.40; H, 6.39. $[\alpha]_{\mathrm{D}}{ }^{24}-17.1\left(c 1.0, \mathrm{CHCl}_{3}\right)$. HPLC analysis (AD-H, 10\% IPA-hexanes, $\left.1.0 \mathrm{~mL} / \mathrm{min}, 254 \mathrm{~nm}\right)$ indicated $92 \%$ ee: $\mathrm{tR}($ minor $)=10.5 \mathrm{~min}$, $\mathrm{tR}($ major $)=30.1 \mathrm{~min}$.

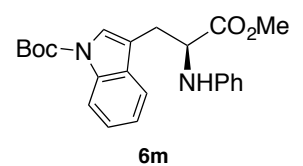

Following the general procedure, a mixture of L-Trp-(Boc)-OMe (5m, $318 \mathrm{mg}, 1.00 \mathrm{mmol}, 1.00$ equiv), phenyl trifluoromethanesulfonate (162 $\mu \mathrm{L}, 1.00 \mathrm{mmol}, 1$ equiv), P1 (43.0 mg, $50.0 \mu \mathrm{mol}$, $5.0 \mathrm{~mol} \%$ ), cesium carbonate ( $977 \mathrm{mg}, 3.00 \mathrm{mmol}, 3.00$ equiv), and 2-methyltetrahydrofuran $(2.00 \mathrm{~mL})$ was stirred at $50{ }^{\circ} \mathrm{C}$ for $2 \mathrm{~h}$. The crude product was purified by automated flashcolumn chromatography with a new column (eluting with $10 \%$ ether-pentane initially, grading to $20 \%$ ether-pentane, linear gradient) to afford $\mathbf{6 m}$ as a white solid. Yield: $378 \mathrm{mg}, 96 \% . \mathrm{mp}=$ 94-96 ${ }^{\circ} \mathrm{C} .{ }^{1} \mathrm{H}$ NMR $\left(400 \mathrm{MHz}, \mathrm{CDCl}_{3}\right) \delta 8.03(\mathrm{~d}, J=7.5 \mathrm{~Hz}, 1 \mathrm{H}), 7.40(\mathrm{~d}, J=7.7 \mathrm{~Hz}, 1 \mathrm{H}), 7.34$ $(\mathrm{s}, 1 \mathrm{H}), 7.22(\mathrm{t}, J=7.7 \mathrm{~Hz}, 1 \mathrm{H}), 7.16-7.05(\mathrm{~m}, 3 \mathrm{H}), 6.66(\mathrm{t}, J=7.3 \mathrm{~Hz}, 1 \mathrm{H}), 6.53(\mathrm{~d}, J=7.7 \mathrm{~Hz}$, 2H), $4.38(\mathrm{t}, J=5.9 \mathrm{~Hz}, 1 \mathrm{H}), 4.17(\mathrm{~s}, 1 \mathrm{H}), 3.55(\mathrm{~s}, 3 \mathrm{H}), 3.16(\mathrm{qd}, J=14.6,5.8 \mathrm{~Hz}, 2 \mathrm{H}), 1.57(\mathrm{~s}$, 9H). ${ }^{13} \mathrm{C}$ NMR $\left(100 \mathrm{MHz}, \mathrm{CDCl}_{3}\right) \delta 173.7,149.7,146.4,135.5,130.6,129.5,124.6,124.3,122.6$, 118.9, 118.6, 115.4, 115.3, 113.7, 83.8, 56.6, 52.3, 28.3, 28.2. IR (neat, $\mathrm{cm}^{-1}$ ): 3349, 2970, 1711, 1251, 1149, 745. Anal. Calcd. for $\mathrm{C}_{23} \mathrm{H}_{26} \mathrm{~N}_{2} \mathrm{O}_{4}: \mathrm{C}, 70.03 ; \mathrm{H}, 6.64$, Found: C, 69.50; H, 6.67. $[\alpha]_{D}{ }^{24}$ +33.3 (c 1.0, $\left.\mathrm{CHCl}_{3}\right)$. HPLC analysis $(\mathrm{OJ}-\mathrm{H}, 10 \% \mathrm{IPA}-$ hexanes, $1.0 \mathrm{~mL} / \mathrm{min}, 254 \mathrm{~nm})$ indicated $93 \%$ ee: $\mathrm{tR}($ major $)=13.4 \mathrm{~min}, \mathrm{tR}($ minor $)=16.7 \mathrm{~min}$.

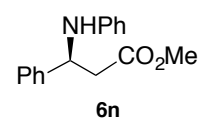

Following the general procedure, a mixture of L- $\beta$-Phe-OMe (5n, $179 \mathrm{mg}, 1.00 \mathrm{mmol}, 1.00$ equiv), phenyl trifluoromethanesulfonate (162 $\mu \mathrm{L}, 1.00 \mathrm{mmol}, 1$ equiv), P1 (43.0 mg, $50.0 \mu \mathrm{mol}$, $5.0 \mathrm{~mol} \%)$, cesium carbonate ( $977 \mathrm{mg}, 3.00 \mathrm{mmol}, 3.00$ equiv), and 2-methyltetrahydrofuran $(2.00 \mathrm{~mL})$ was stirred at $50{ }^{\circ} \mathrm{C}$ for $14 \mathrm{~h}$. The crude product was purified by automated flashcolumn chromatography with a new column (eluting with 5\% ether-pentane initially, grading to $10 \%$ ether-pentane, linear gradient) to afford $\mathbf{6 n}$ as a white solid. Yield: $198 \mathrm{mg}, 78 \% \mathrm{mp}=$ 102-103 ${ }^{\circ} \mathrm{C} .{ }^{1} \mathrm{H}$ NMR (400 MHz, $\mathrm{CDCl}_{3}$ ) $\delta$ 7.47-7.41 (m, 2H), 7.40-7.36 (m, 2H), 7.33-7.27 (m, 
1H), 7.20-7.13 (m, 2H), $6.74(\mathrm{t}, J=7.3 \mathrm{~Hz}, 1 \mathrm{H}), 6.63(\mathrm{~d}, J=7.7 \mathrm{~Hz}, 2 \mathrm{H}), 4.91(\mathrm{t}, J=6.7 \mathrm{~Hz}, 1 \mathrm{H})$, $4.61(\mathrm{~s}, 1 \mathrm{H}), 3.70(\mathrm{~s}, 3 \mathrm{H}), 2.88(\mathrm{dd}, J=6.7,1.9 \mathrm{~Hz}, 2 \mathrm{H}) .{ }^{13} \mathrm{C}$ NMR $\left(100 \mathrm{MHz}, \mathrm{CDCl}_{3}\right) \delta 171.6$, 146.8, 142.3, 129.2, 128.9, 127.6, 126.3, 117.9, 113.8, 55.0, 52.0, 42.7. IR (neat, $\mathrm{cm}^{-1}$ ): 3375, 1716, 1603, 1289, 749, 693. Anal. Calcd. for $\mathrm{C}_{16} \mathrm{H}_{17} \mathrm{NO}_{2}: \mathrm{C}, 75.27 ; \mathrm{H}, 6.71$, Found: $\mathrm{C}, 75.01 ; \mathrm{H}$, 6.73. $[\alpha]_{\mathrm{D}}{ }^{24}+1.3\left(c 1.0, \mathrm{CHCl}_{3}\right)$. HPLC analysis (OD-H, 5\% IPA-hexanes, $\left.0.8 \mathrm{~mL} / \mathrm{min}, 254 \mathrm{~nm}\right)$ indicated $99 \%$ ee: $\mathrm{tR}($ major $)=19.8 \mathrm{~min}, \mathrm{tR}($ minor $)=21.6 \mathrm{~min}$.

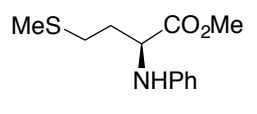

Following the general procedure, a mixture of L-Met-OMe (5o, $163 \mathrm{mg}, 1.00 \mathrm{mmol}, 1.00$ equiv), phenyl trifluoromethanesulfonate $(162 \mu \mathrm{L}, 1.00 \mathrm{mmol}, 1$ equiv), P1 (43.0 mg, $50.0 \mu \mathrm{mol}, 5.0$ mol\%), cesium carbonate $(977 \mathrm{mg}, 3.00 \mathrm{mmol}, 3.00$ equiv), and 2-methyltetrahydrofuran $(2.00$ $\mathrm{mL}$ ) was stirred at $50{ }^{\circ} \mathrm{C}$ for $2 \mathrm{~h}$. The crude product was purified by automated flash-column chromatography with a new column (eluting with 5\% EtOAc-pentane) to afford $\mathbf{6 0}$ as a yellow oil. Yield: $202 \mathrm{mg}, 85 \%$. ${ }^{1} \mathrm{H}$ NMR $\left(400 \mathrm{MHz}, \mathrm{CDCl}_{3}\right) \delta 7.19(\mathrm{dd}, J=8.6,7.3 \mathrm{~Hz}, 2 \mathrm{H}), 6.76$ (tt, $J$ $=7.3,1.1 \mathrm{~Hz}, 1 \mathrm{H}), 6.67(\mathrm{dd}, J=8.7,1.1 \mathrm{~Hz}, 2 \mathrm{H}), 4.28(\mathrm{dd}, J=7.6,5.3 \mathrm{~Hz}, 1 \mathrm{H}), 4.24($ br s, $1 \mathrm{H})$, $3.73(\mathrm{~s}, 3 \mathrm{H}), 2.64(\mathrm{t}, J=7.2 \mathrm{~Hz}, 2 \mathrm{H}), 2.20-2.12(\mathrm{~m}, 1 \mathrm{H}), 2.11(\mathrm{~s}, 3 \mathrm{H}), 2.03(\mathrm{dt}, J=14.1,7.1 \mathrm{~Hz}$, $1 \mathrm{H}) .{ }^{13} \mathrm{C}$ NMR $\left(100 \mathrm{MHz}, \mathrm{CDCl}_{3}\right) \delta 174.2,146.7,129.4,118.5,113.6,55.5,52.3,32.3,30.3,15.5$. IR (neat, $\mathrm{cm}^{-1}$ ): $3379,2916,1733,1602,1506,1167,748,692$. Anal. Calcd. for $\mathrm{C}_{12} \mathrm{H}_{17} \mathrm{NO}_{2} \mathrm{~S}: \mathrm{C}$, 60.22; H, 7.16, Found: C, 60.34; H, 6.97. $[\alpha]_{\mathrm{D}}{ }^{24}-21.9\left(c 1.0, \mathrm{CHCl}_{3}\right)$. HPLC analysis $(\mathrm{OJ}-\mathrm{H}, 10 \%$ IPA-hexanes, $1.0 \mathrm{~mL} / \mathrm{min}, 254 \mathrm{~nm}$ ) indicated $80 \%$ ee: tR (minor) $=20.3 \mathrm{~min}$, tR (major) $=29.6$ $\min$.

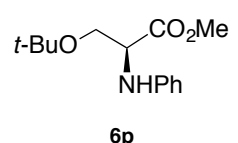

Following the general procedure, a mixture of L-Ser- $(t$-Bu)-OMe (5p, $175 \mathrm{mg}, 1.00 \mathrm{mmol}, 1.00$ equiv), phenyl trifluoromethanesulfonate $(162 \mu \mathrm{L}, 1.00 \mathrm{mmol}, 1$ equiv), P1 (43.0 mg, $50.0 \mu \mathrm{mol}$, $5.0 \mathrm{~mol} \%)$, cesium carbonate $(977 \mathrm{mg}, 3.00 \mathrm{mmol}, 3.00$ equiv), and 2-methyltetrahydrofuran $(2.00 \mathrm{~mL})$ was stirred at $50{ }^{\circ} \mathrm{C}$ for $2 \mathrm{~h}$. The crude product was purified by automated flashcolumn chromatography (eluting with $1 \%$ acetone-pentane initially, grading to $10 \%$ acetonepentane, linear gradient) to afford $\mathbf{6 p}$ as a white solid. Yield: $233 \mathrm{mg}, 93 \%$. mp $=47-50{ }^{\circ} \mathrm{C} .{ }^{1} \mathrm{H}$ NMR $\left(400 \mathrm{MHz}, \mathrm{CDCl}_{3}\right) \delta 7.21-7.14(\mathrm{~m}, 2 \mathrm{H}), 6.75(\mathrm{t}, J=7.3 \mathrm{~Hz}, 1 \mathrm{H}), 6.67-6.60(\mathrm{~m}, 2 \mathrm{H}), 4.20$ $(\mathrm{t}, J=4.1 \mathrm{~Hz}, 1 \mathrm{H}), 3.79(\mathrm{dd}, J=8.8,4.0 \mathrm{~Hz}, 1 \mathrm{H}), 3.73(\mathrm{~s}, 3 \mathrm{H}), 3.69(\mathrm{dd}, J=8.8,4.2 \mathrm{~Hz}, 1 \mathrm{H})$, 1.17 (s, 9H). ${ }^{13} \mathrm{C}$ NMR $\left(100 \mathrm{MHz}, \mathrm{CDCl}_{3}\right) \delta 172.9,146.9,129.4,118.5,113.8,73.6,62.5,57.4$, 52.2, 27.5. IR (neat, $\mathrm{cm}^{-1}$ ): 3401, 2978, 1748, 1604, 1508, 1147, 1102, 755. Anal. Calcd. for $\mathrm{C}_{14} \mathrm{H}_{21} \mathrm{NO}_{3}$ : C, 66.91; H, 8.42, Found: C, 67.20; H, 8.48. $[\alpha]_{\mathrm{D}}{ }^{24}-10.7$ (c 1.0, $\left.\mathrm{CHCl}_{3}\right)$. HPLC analysis (OJ-H, 10\% IPA-hexanes, $1.0 \mathrm{~mL} / \mathrm{min}, 254 \mathrm{~nm}$ ) indicated $71 \%$ ee: tR (minor) $=7.1 \mathrm{~min}$, $\operatorname{tR}($ major $)=10.9 \mathrm{~min}$.

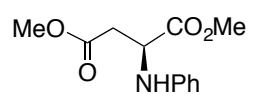

$6 q$

Following the general procedure, a mixture of L-Asp-(Me)-OMe (5q, $161 \mathrm{mg}, 1.00 \mathrm{mmol}, 1.00$ equiv), phenyl trifluoromethanesulfonate $(162 \mu \mathrm{L}, 1.00 \mathrm{mmol}, 1$ equiv), P1 (43.0 mg, $50.0 \mu \mathrm{mol}$, $5.0 \mathrm{~mol} \%)$, cesium carbonate $(977 \mathrm{mg}, 3.00 \mathrm{mmol}, 3.00$ equiv), and 2-methyltetrahydrofuran $(2.00 \mathrm{~mL})$ was stirred at $50{ }^{\circ} \mathrm{C}$ for $2 \mathrm{~h}$. The crude product was purified by automated flashcolumn chromatography (eluting with $6 \%$ EtOAc-hexanes initially, grading to $50 \%$ EtOAchexanes, linear gradient) to afford $\mathbf{6 q}$ as a yellow oil. Yield: $231 \mathrm{mg}, 98 \%$. ${ }^{1} \mathrm{H}$ NMR (400 MHz, 
$\left.\mathrm{CDCl}_{3}\right) \delta 7.23-7.15(\mathrm{~m}, 2 \mathrm{H}), 6.77(\mathrm{t}, J=7.3 \mathrm{~Hz}, 1 \mathrm{H}), 6.67(\mathrm{~d}, J=7.7 \mathrm{~Hz}, 2 \mathrm{H}), 4.56-4.42(\mathrm{~m}, 2 \mathrm{H})$, $3.75(\mathrm{~s}, 3 \mathrm{H}), 3.70(\mathrm{~s}, 3 \mathrm{H}), 2.89(\mathrm{~d}, J=5.0 \mathrm{~Hz}, 2 \mathrm{H}) .{ }^{13} \mathrm{C} \mathrm{NMR}\left(100 \mathrm{MHz}, \mathrm{CDCl}_{3}\right) \delta 172.8,171.0$, 146.2, 129.4, 118.7, 113.7, 53.4, 52.6, 52.0, 37.1. IR (neat, $\mathrm{cm}^{-1}$ ): 3383, 2952, 1729, 1602, 1168 , 749. Anal. Calcd. for $\mathrm{C}_{12} \mathrm{H}_{15} \mathrm{NO}_{4}$ : C, 60.75; H, 6.37, Found: C, 61.03; H, 6.46. $[\alpha]_{\mathrm{D}}{ }^{24}+6.8(c 1.0$, $\left.\mathrm{CHCl}_{3}\right)$. HPLC analysis (OJ-H, $10 \% \mathrm{IPA}-$ hexanes, $\left.1.0 \mathrm{~mL} / \mathrm{min}, 254 \mathrm{~nm}\right)$ indicated $74 \%$ ee: $\mathrm{tR}$ $(\operatorname{minor})=34.0 \mathrm{~min}, \mathrm{tR}($ major $)=58.1 \mathrm{~min}$.

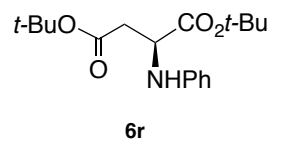

Following the general procedure, a mixture of L-Asp- $(t-\mathrm{Bu})-\mathrm{O} t$-Bu (5r, $245 \mathrm{mg}, 1.00 \mathrm{mmol}, 1.00$ equiv), phenyl trifluoromethanesulfonate $(162 \mu \mathrm{L}, 1.00 \mathrm{mmol}, 1$ equiv), P1 (43.0 mg, $50.0 \mu \mathrm{mol}$, $5.0 \mathrm{~mol} \%$ ), cesium carbonate $(977 \mathrm{mg}, 3.00 \mathrm{mmol}, 3.00$ equiv), and 2-methyltetrahydrofuran $(2.00 \mathrm{~mL})$ was stirred at $50{ }^{\circ} \mathrm{C}$ for $2 \mathrm{~h}$. The crude product was purified by automated flashcolumn chromatography (eluting with $1 \%$ acetone-hexanes initially, grading to $10 \%$ acetonehexanes, linear gradient) to afford $6 \mathbf{r}$ as a white solid. Yield: $290 \mathrm{mg}, 90 \% \mathrm{mp}=72-74{ }^{\circ} \mathrm{C} .{ }^{1} \mathrm{H}$ NMR (400 MHz, $\left.\mathrm{CDCl}_{3}\right) \delta$ 7.22-7.14 (m, 2H), 6.78-6.71 (m, 1H), 6.68-6.63 (m, 2H), 4.49 (app $\mathrm{d}, J=8.4 \mathrm{~Hz}, 1 \mathrm{H}), 4.28(\mathrm{dt}, J=8.4,5.6 \mathrm{~Hz}, 1 \mathrm{H}), 2.75(\mathrm{~d}, J=5.6 \mathrm{~Hz}, 2 \mathrm{H}), 1.46(\mathrm{~s}, 9 \mathrm{H}), 1.45(\mathrm{~s}$, 9H). ${ }^{13} \mathrm{C}$ NMR $\left(100 \mathrm{MHz}, \mathrm{CDCl}_{3}\right) \delta 171.6,169.9,146.8,129.3,118.4,113.8,82.1,81.3,54.1$, 38.6, 28.2, 28.0. IR (neat, $\mathrm{cm}^{-1}$ ): 3400, 2977, 1732, 1140, 747, 696. Anal. Calcd. for $\mathrm{C}_{18} \mathrm{H}_{27} \mathrm{NO}_{4}$ : C, 67.26; H, 8.47, Found: C, 67.42; H, 8.54. $[\alpha]_{\mathrm{D}}{ }^{24}-1.1\left(c 1.0, \mathrm{CHCl}_{3}\right)$. HPLC analysis $(\mathrm{OJ}-\mathrm{H}$, $10 \%$ IPA-hexanes, $1.0 \mathrm{~mL} / \mathrm{min}, 254 \mathrm{~nm}$ ) indicated $80 \%$ ee: tR (minor) $=4.7 \mathrm{~min}$, tR (major) $=$ $6.0 \mathrm{~min}$.

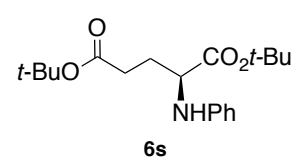

Following the general procedure, a mixture of L-Glu- $(t-\mathrm{Bu})-\mathrm{O} t-\mathrm{Bu}(\mathbf{5 s}, 259 \mathrm{mg}, 1.00 \mathrm{mmol}, 1.00$ equiv), phenyl trifluoromethanesulfonate $(162 \mu \mathrm{L}, 1.00 \mathrm{mmol}, 1$ equiv), P1 (43.0 mg, $50.0 \mu \mathrm{mol}$, $5.0 \mathrm{~mol} \%$ ), cesium carbonate ( $977 \mathrm{mg}, 3.00 \mathrm{mmol}, 3.00$ equiv), and 2-methyltetrahydrofuran $(2.00 \mathrm{~mL})$ was stirred at $50{ }^{\circ} \mathrm{C}$ for $2 \mathrm{~h}$. The crude product was purified by automated flashcolumn chromatography (eluting with $1 \%$ acetone-pentane initially, grading to $10 \%$ acetonepentane, linear gradient) to afford $\mathbf{6 s}$ as a yellow solid. Yield: $313 \mathrm{mg}, 93 \% \mathrm{mp}=61-64{ }^{\circ} \mathrm{C} .{ }^{1} \mathrm{H}$ $\operatorname{NMR}\left(400 \mathrm{MHz}, \mathrm{CDCl}_{3}\right) \delta 7.16(\mathrm{dd}, J=8.5,7.3 \mathrm{~Hz}, 2 \mathrm{H}), 6.75-6.70(\mathrm{~m}, 1 \mathrm{H}), 6.65-6.60(\mathrm{~m}, 2 \mathrm{H})$, 4.23 (br s, 1H), 4.00 (dd, $J=7.6,5.5 \mathrm{~Hz}, 1 \mathrm{H}), 2.47-2.31(\mathrm{~m}, 2 \mathrm{H}), 2.17-1.95(\mathrm{~m}, 2 \mathrm{H}), 1.45$ (s, 9H), $1.44(\mathrm{~s}, 9 \mathrm{H}) .{ }^{13} \mathrm{C} \mathrm{NMR}\left(100 \mathrm{MHz}, \mathrm{CDCl}_{3}\right) \delta 172.9,172.4,147.1,129.3,118.2,113.6,81.8$, 80.6, 56.8, 31.7, 28.2, 28.1 (2C). IR (neat, $\mathrm{cm}^{-1}$ ): 3363, 2977, 1722, 1704, 1605, 1155, 752, 693. Anal. Calcd. for $\mathrm{C}_{19} \mathrm{H}_{29} \mathrm{NO}_{4}$ : C, 68.03; H, 8.71, Found: C, 68.03; H, 8.70. [ $\left.\alpha\right]_{\mathrm{D}}{ }^{24}-27.7$ (c 1.0, $\left.\mathrm{CHCl}_{3}\right)$. HPLC analysis (OJ-H, $5 \%$ IPA-hexanes, $\left.0.8 \mathrm{~mL} / \mathrm{min}, 254 \mathrm{~nm}\right)$ indicated $97 \%$ ee: $\mathrm{tR}$ $($ minor $)=6.4 \mathrm{~min}, \mathrm{tR}($ major $)=7.8 \mathrm{~min}$.

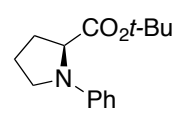

$6 \mathrm{t}$

Following the general procedure, a mixture of L-Pro-Ot-Bu (5t, $171 \mathrm{mg}, 1.00 \mathrm{mmol}, 1.00$ equiv), phenyl trifluoromethanesulfonate $(162 \mu \mathrm{L}, 1.00 \mathrm{mmol}, 1$ equiv), P2 (46.0 mg, $50.0 \mu \mathrm{mol}, 5.0$ mol\%), cesium carbonate ( $977 \mathrm{mg}, 3.00 \mathrm{mmol}, 3.00$ equiv), and 2-methyltetrahydrofuran (2.00 $\mathrm{mL}$ ) was stirred at $80{ }^{\circ} \mathrm{C}$ for $14 \mathrm{~h}$. The crude product was purified by automated flash-column chromatography with a new column (eluting with $1 \%$ ether-pentane initially, grading to $10 \%$ ether-pentane, linear gradient) to afford $6 \mathrm{t}$ as a clear oil. Yield: $177 \mathrm{mg}, 72 \%$. ${ }^{1} \mathrm{H}$ NMR (400 
$\left.\mathrm{MHz}, \mathrm{CDCl}_{3}\right) \delta 7.30(\mathrm{td}, J=7.3,2.0 \mathrm{~Hz}, 2 \mathrm{H}), 6.78(\mathrm{tt}, J=7.2,1.1 \mathrm{~Hz}, 1 \mathrm{H}), 6.64(\mathrm{~d}, J=7.9 \mathrm{~Hz}$, 2H), 4.21 (dd, $J=8.4,2.4 \mathrm{~Hz}, 1 \mathrm{H}), 3.60$ (dt, $J=7.9,4.1 \mathrm{~Hz}, 1 \mathrm{H}), 3.44$ (q, $J=7.8 \mathrm{~Hz}, 1 \mathrm{H}), 2.36-$ $2.05(\mathrm{~m}, 4 \mathrm{H}), 1.51(\mathrm{~s}, 9 \mathrm{H}) .{ }^{13} \mathrm{C} \mathrm{NMR}\left(100 \mathrm{MHz}, \mathrm{CDCl}_{3}\right) \delta 173.7,146.8,129.1,116.3,111.9,80.9$, 61.6, 48.1, 30.7, 28.0, 23.8. IR (neat, $\mathrm{cm}^{-1}$ ): 2975, 1737, 1598, 1505, 1365, 1144, 745, 670. Anal. Calcd. for $\mathrm{C}_{15} \mathrm{H}_{21} \mathrm{NO}_{2}$ : C, 72.84; H, 8.56, Found: C, 72.75; H, 8.53. $[\alpha]_{\mathrm{D}}{ }^{24}-53.0\left(c 1.0, \mathrm{CHCl}_{3}\right)$. HPLC analysis (OJ-H, 5\% IPA-hexanes, $0.8 \mathrm{~mL} / \mathrm{min}, 254 \mathrm{~nm}$ ) indicated $44 \%$ ee: tR (minor) $=$ $7.0 \mathrm{~min}, \mathrm{tR}$ (major) $=10.3 \mathrm{~min}$.

Repeating the reaction with stirring at $50{ }^{\circ} \mathrm{C}$ for $2 \mathrm{~h}$ afforded $\mathbf{6 t}$ as a clear oil. Yield $20.6 \mathrm{mg}, 9 \%$. HPLC analysis (OJ-H, 5\% IPA-hexanes, $0.8 \mathrm{~mL} / \mathrm{min}, 254 \mathrm{~nm}$ ) indicated $97 \%$ ee: $\mathrm{tR}$ (minor) = $7.2 \mathrm{~min}, \mathrm{tR}$ (major) $=10.7 \mathrm{~min}$.

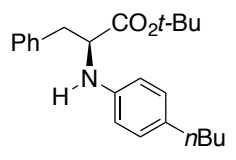

8 a

Following the general procedure, a mixture of L-Phe-Ot-Bu (5a, $221 \mathrm{mg}, 1.00 \mathrm{mmol}, 1.00$ equiv), 4-n-butylphenyl trifluoromethanesulfonate (7a, $282 \mathrm{mg}, 1.00 \mathrm{mmol}, 1$ equiv), P1 (43.0 mg, 50.0 $\mu \mathrm{mol}, \quad 5.0 \mathrm{~mol} \%)$, cesium carbonate $(977 \mathrm{mg}, 3.00 \mathrm{mmol}, 3.00$ equiv), and 2methyltetrahydrofuran $(2.00 \mathrm{~mL})$ was stirred at $50{ }^{\circ} \mathrm{C}$ for $2 \mathrm{~h}$. The crude product was purified by automated flash-column chromatography (eluting with $5 \%$ ether-hexanes initially, grading to $40 \%$ ether-hexanes, linear gradient) to afford $\mathbf{8 a}$ as a yellow solid. Yield: $318 \mathrm{mg}, 90 \% . \mathrm{mp}=$ 50-52 ${ }^{\circ} \mathrm{C} .{ }^{1} \mathrm{H}$ NMR $\left(400 \mathrm{MHz}, \mathrm{CDCl}_{3}\right) \delta$ 7.41-7.28 (m, 5H), 7.11-7.05 (m, 2H), 6.69-6.62 (m, $2 \mathrm{H}), 4.32(\mathrm{t}, J=6.4 \mathrm{~Hz}, 1 \mathrm{H}), 4.19(\mathrm{br} \mathrm{s}, 1 \mathrm{H}), 3.24-3.12(\mathrm{~m}, 2 \mathrm{H}), 2.64-2.55(\mathrm{~m}, 2 \mathrm{H}), 1.65(\mathrm{tt}, J=$ $8.9,6.8 \mathrm{~Hz}, 2 \mathrm{H}), 1.43($ br s, $11 \mathrm{H}), 1.02(\mathrm{t}, J=7.3 \mathrm{~Hz}, 3 \mathrm{H}) .{ }^{13} \mathrm{C}$ NMR $\left(100 \mathrm{MHz}, \mathrm{CDCl}_{3}\right) \delta 172.4$, $144.5,136.9,132.6,129.5,129.1,128.3,126.8,113.8,81.5,58.6,38.8,34.8,34.0,27.9,22.3$, 14.0. IR (neat, $\mathrm{cm}^{-1}$ ): 3368, 2924, 1726, 1518, 1149, 699. Anal. Calcd. for $\mathrm{C}_{23} \mathrm{H}_{31} \mathrm{NO}_{2}: \mathrm{C}, 77.80$; H, 6.32, Found: C, 77.40; H, 6.39. $[\alpha]_{\mathrm{D}}{ }^{24}+18.1\left(c 1.0, \mathrm{CHCl}_{3}\right)$. HPLC analysis $(\mathrm{OJ}-\mathrm{H}, 5 \%$ IPAhexanes, $0.8 \mathrm{~mL} / \mathrm{min}, 254 \mathrm{~nm}$ ) indicated $90 \%$ ee: $\mathrm{tR}($ minor $)=8.4 \mathrm{~min}$, tR (major) $=10.6 \mathrm{~min}$.

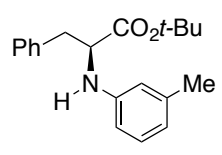

$8 b$

Following the general procedure, a mixture of L-Phe-Ot-Bu (5a, $221 \mathrm{mg}, 1.00 \mathrm{mmol}, 1.00$ equiv), $m$-tolyl trifluoromethanesulfonate ( $7 \mathbf{b}, 282 \mathrm{mg}, 1.00 \mathrm{mmol}, 1$ equiv), P1 (43.0 mg, $50.0 \mu \mathrm{mol}, 5.0$ $\mathrm{mol} \%$ ), cesium carbonate ( $977 \mathrm{mg}, 3.00 \mathrm{mmol}, 3.00$ equiv), and 2-methyltetrahydrofuran (2.00 $\mathrm{mL}$ ) was stirred at $50{ }^{\circ} \mathrm{C}$ for $2 \mathrm{~h}$. The crude product was purified by automated flash-column chromatography (eluting with $2 \%$ EtOAc-hexanes initially, grading to $20 \%$ EtOAc-hexanes, linear gradient) to afford $\mathbf{8 b}$ as a clear oil. Yield: $288 \mathrm{mg}, 93 \% .{ }^{1} \mathrm{H}$ NMR $\left(400 \mathrm{MHz}, \mathrm{CDCl}_{3}\right) \delta$ 7.40-7.33 (m, 2H), 7.33-7.26 (m, 3H), $7.13(\mathrm{td}, J=7.4,1.1 \mathrm{~Hz}, 1 \mathrm{H}), 6.63(\mathrm{~d}, J=7.5 \mathrm{~Hz}, 1 \mathrm{H})$, 6.51 (app d, $J=7.6 \mathrm{~Hz}, 2 \mathrm{H}), 4.31$ (t, $J=6.4 \mathrm{~Hz}, 1 \mathrm{H}), 4.23$ (br s, 1H), $3.16(\mathrm{~d}, J=6.4 \mathrm{~Hz}, 2 \mathrm{H})$, $2.34(\mathrm{~s}, 3 \mathrm{H}), 1.42(\mathrm{~s}, 9 \mathrm{H}) .{ }^{13} \mathrm{C} \mathrm{NMR}\left(100 \mathrm{MHz}, \mathrm{CDCl}_{3}\right) \delta 172.4,146.7,139.0,136.8,129.6,129.2$, 128.4, 126.9, 119.2, 114.5, 110.8, 81.7, 58.3, 38.8, 28.0, 21.7. IR (neat, $\mathrm{cm}^{-1}$ ): 3366, 2923, 1716, 1605, 1148, 699. Anal. Calcd. for $\mathrm{C}_{20} \mathrm{H}_{25} \mathrm{NO}_{2}$ : C, 77.14; H, 8.09, Found: C, 77.42; H, 8.08. $[\alpha]_{\mathrm{D}}{ }^{24}$ +14.9 (c 1.0, $\left.\mathrm{CHCl}_{3}\right)$. HPLC analysis $(\mathrm{OJ}-\mathrm{H}, 2 \%$ IPA-hexanes, $0.8 \mathrm{~mL} / \mathrm{min}, 254 \mathrm{~nm})$ indicated $87 \%$ ee: $\mathrm{tR}($ minor $)=16.1 \mathrm{~min}, \mathrm{tR}($ major $)=20.3 \mathrm{~min}$. 


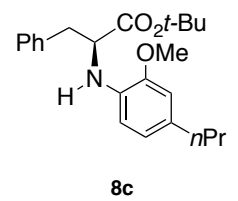

Following the general procedure, a mixture of L-Phe-Ot-Bu (5a, $221 \mathrm{mg}, 1.00 \mathrm{mmol}, 1.00$ equiv), 2-methoxy-4-propylphenyl trifluoromethanesulfonate (7c, $298 \mathrm{mg}, 1.00 \mathrm{mmol}, 1$ equiv), P1 (43.0 $\mathrm{mg}, 50.0 \mu \mathrm{mol}, 5.0 \mathrm{~mol} \%$ ), cesium carbonate (977 $\mathrm{mg}, 3.00 \mathrm{mmol}, 3.00$ equiv), and 2methyltetrahydrofuran $(2.00 \mathrm{~mL})$ was stirred at $50{ }^{\circ} \mathrm{C}$ for $2 \mathrm{~h}$. The crude product was purified by automated flash-column chromatography (eluting with $2 \%$ ether-hexanes initially, grading to $20 \%$ ether-hexanes, linear gradient) to afford $8 \mathrm{c}$ as a clear oil. Yield: $344 \mathrm{mg}, 93 \% .{ }^{1} \mathrm{H}$ NMR $\left(400 \mathrm{MHz}, \mathrm{CDCl}_{3}\right) \delta 7.40-7.27(\mathrm{~m}, 5 \mathrm{H}), 6.76-6.68(\mathrm{~m}, 2 \mathrm{H}), 6.61(\mathrm{~d}, J=7.9 \mathrm{~Hz}, 1 \mathrm{H}), 4.80(\mathrm{br} \mathrm{s}$, $1 \mathrm{H}), 4.31(\mathrm{t}, J=6.6 \mathrm{~Hz}, 1 \mathrm{H}), 3.90(\mathrm{~s}, 3 \mathrm{H}), 3.21(\mathrm{qd}, J=13.6,6.6 \mathrm{~Hz}, 2 \mathrm{H}), 2.63-2.55(\mathrm{~m}, 2 \mathrm{H})$, $1.70(\mathrm{dq}, J=14.8,7.4 \mathrm{~Hz}, 2 \mathrm{H}), 1.41(\mathrm{~s}, 9 \mathrm{H}), 1.03(\mathrm{t}, J=7.3 \mathrm{~Hz}, 3 \mathrm{H}) .{ }^{13} \mathrm{C} \mathrm{NMR}(100 \mathrm{MHz}$, $\left.\mathrm{CDCl}_{3}\right) \delta 172.5,147.2,137.1,134.5,132.0,129.5,128.3,126.7,120.6,110.7,110.5,81.3,58.5$, 55.5, 39.0, 37.8, 27.9, 24.9, 13.9. IR (neat, $\mathrm{cm}^{-1}$ ): 3420, 2930, 1728, 1521, 1142, 699. Anal. Calcd. for $\mathrm{C}_{23} \mathrm{H}_{31} \mathrm{NO}_{3}$ : C, 74.76; $\mathrm{H}, 8.46$, Found: $\mathrm{C}, 74.48 ; \mathrm{H}, 8.40 . \quad[\alpha]_{\mathrm{D}}{ }^{24}+7.8\left(c 1.0, \mathrm{CHCl}_{3}\right)$. HPLC analysis $(\mathrm{OJ}-\mathrm{H}, 2 \% \mathrm{IPA}-$ hexanes, $0.8 \mathrm{~mL} / \mathrm{min}, 254 \mathrm{~nm}$ ) indicated $88 \%$ ee: tR (minor) $=8.9 \mathrm{~min}$, $\operatorname{tR}($ major $)=10.8 \mathrm{~min}$.

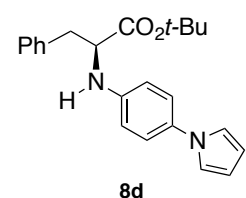

Following the general procedure, a mixture of L-Phe-Ot-Bu (5a, $221 \mathrm{mg}, 1.00 \mathrm{mmol}, 1.00$ equiv), 4-(1H-pyrrol-1-yl)phenyl trifluoromethanesulfonate (7d, $291 \mathrm{mg}, 1.00 \mathrm{mmol}, 1$ equiv), P1 (43.0 $\mathrm{mg}, 50.0 \mu \mathrm{mol}, 5.0 \mathrm{~mol} \%$ ), cesium carbonate (977 $\mathrm{mg}, 3.00 \mathrm{mmol}, 3.00$ equiv), and 2methyltetrahydrofuran $(2.00 \mathrm{~mL})$ was stirred at $50{ }^{\circ} \mathrm{C}$ for $2 \mathrm{~h}$. The crude product was purified by automated flash-column chromatography with a new column (eluting with 5\% acetone-pentane initially, grading to $10 \%$ acetone-pentane, linear gradient) to afford $\mathbf{8 d}$ as a white solid. Yield: $316 \mathrm{mg}, 87 \% . \mathrm{mp}=102-103{ }^{\circ} \mathrm{C} .{ }^{1} \mathrm{H}$ NMR $\left(400 \mathrm{MHz}, \mathrm{CDCl}_{3}\right) \delta 7.39-7.22(\mathrm{~m}, 7 \mathrm{H}), 7.03-7.01(\mathrm{~m}$, 2H), 6.72-6.67 (m, 2H), 6.37-6.34 (m, 2H), $4.35(\mathrm{br} \mathrm{s}, 1 \mathrm{H}), 4.30(\mathrm{t}, J=6.4 \mathrm{~Hz}, 1 \mathrm{H}), 3.17$ (d, $J=$ $6.3 \mathrm{~Hz}, 2 \mathrm{H}), 1.42(\mathrm{~s}, 9 \mathrm{H}) .{ }^{13} \mathrm{C} \mathrm{NMR}\left(100 \mathrm{MHz}, \mathrm{CDCl}_{3}\right) \delta 172.2,144.9,136.6,132.7,129.6,128.5$, 127.0, 122.4, 119.7, 114.3, 109.5, 82.1, 58.5, 38.7, 28.1. IR (neat, $\mathrm{cm}^{-1}$ ): 3355, 2977, 1703, 1522, 699. Anal. Calcd. for $\mathrm{C}_{23} \mathrm{H}_{26} \mathrm{~N}_{2} \mathrm{O}_{2}$ : C, 76.21; H, 7.23, Found: C, 76.36; H, 7.30. $[\alpha]_{\mathrm{D}}{ }^{24}+28.3$ (c 1.0, $\left.\mathrm{CHCl}_{3}\right)$. HPLC analysis (OJ-H, 10\% IPA-hexanes, $1.0 \mathrm{~mL} / \mathrm{min}, 254 \mathrm{~nm}$ ) indicated $91 \%$ ee: tR $($ major $)=38.3 \mathrm{~min}, \mathrm{tR}($ minor $)=50.5 \mathrm{~min}$.

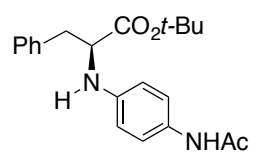

$8 \mathrm{e}$

Following the general procedure, a mixture of L-Phe-Ot-Bu (5a, $221 \mathrm{mg}, 1.00 \mathrm{mmol}, 1.00$ equiv), 4-acetamidophenyl trifluoromethanesulfonate (7e, $283 \mathrm{mg}, 1.00 \mathrm{mmol}, 1$ equiv), P1 (43.0 mg, $50.0 \mu \mathrm{mol}, 5.0 \mathrm{~mol} \%$ ), cesium carbonate $(977 \mathrm{mg}, 3.00 \mathrm{mmol}, 3.00$ equiv), and 2methyltetrahydrofuran $(2.00 \mathrm{~mL})$ was stirred at $50{ }^{\circ} \mathrm{C}$ for $2 \mathrm{~h}$. The crude product was purified by automated flash-column chromatography (eluting with $8 \%$ acetone-hexanes initially, grading to $66 \%$ acetone-hexanes, linear gradient) to afford $8 \mathrm{e}$ as a white solid. Yield: $312 \mathrm{mg}, 88 \%$. $\mathrm{mp}=$ $148-149{ }^{\circ} \mathrm{C} .{ }^{1} \mathrm{H}$ NMR $\left(400 \mathrm{MHz}, \mathrm{CDCl}_{3}\right) \delta 7.99(\mathrm{~s}, 1 \mathrm{H}), 7.35-7.18(\mathrm{~m}, 7 \mathrm{H}), 6.54(\mathrm{~d}, J=8.8 \mathrm{~Hz}$, $2 \mathrm{H}), 4.22(\mathrm{t}, J=6.4 \mathrm{~Hz}, 1 \mathrm{H}), 4.19(\mathrm{br} \mathrm{s}, 1 \mathrm{H}), 3.09(\mathrm{dd}, J=6.4,2.2 \mathrm{~Hz}, 2 \mathrm{H}), 2.07(\mathrm{~s}, 3 \mathrm{H}), 1.36(\mathrm{~s}$, 9H). ${ }^{13} \mathrm{C}$ NMR $\left(100 \mathrm{MHz}, \mathrm{CDCl}_{3}\right) \delta 172.5,168.6,143.5,136.6,129.5,129.3,128.4,126.8,122.1$, 
113.8, 81.8, 58.5, 38.6, 27.9, 24.1. IR (neat, $\mathrm{cm}^{-1}$ ): 3301, 1722, 1670, 1517, 1146, 826, 693. HRMS. Calcd. for $\mathrm{C}_{21} \mathrm{H}_{27} \mathrm{~N}_{2} \mathrm{O}_{3}$, [M+H]: 355.2016, Found: $[\mathrm{M}+\mathrm{H}]: 355.2024$. $[\alpha]_{\mathrm{D}}{ }^{24}+21.4(c 1.0$, $\mathrm{CHCl}_{3}$ ). HPLC analysis (AD-H, 10\% IPA-hexanes, $1.0 \mathrm{~mL} / \mathrm{min}, 254 \mathrm{~nm}$ ) indicated $86 \%$ ee: tR $($ minor $)=17.4 \mathrm{~min}, \mathrm{tR}($ major $)=23.7 \mathrm{~min}$.

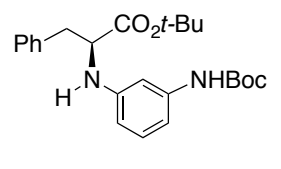

Following the general procedure, a mixture of L-Phe-Ot-Bu (5a, $221 \mathrm{mg}, 1.00 \mathrm{mmol}, 1.00$ equiv), 3-((tert-butoxycarbonyl)amino)phenyl trifluoromethanesulfonate (7f, $341 \mathrm{mg}, 1.00 \mathrm{mmol}, 1$ equiv), P1 (43.0 mg, $50.0 \mu \mathrm{mol}, 5.0 \mathrm{~mol} \%$ ), cesium carbonate ( $977 \mathrm{mg}, 3.00 \mathrm{mmol}, 3.00$ equiv), and 2-methyltetrahydrofuran $(2.00 \mathrm{~mL})$ was stirred at $50{ }^{\circ} \mathrm{C}$ for $2 \mathrm{~h}$. The crude product was purified by automated flash-column chromatography with a new column (eluting with $10 \%$ etherpentane initially, grading to $20 \%$ ether-pentane, linear gradient) to afford $\mathbf{8 f}$ as a white solid. Yield: $404 \mathrm{mg}, 98 \% . \mathrm{mp}=86-88{ }^{\circ} \mathrm{C} .{ }^{1} \mathrm{H}$ NMR $\left(400 \mathrm{MHz}, \mathrm{CDCl}_{3}\right) \delta 7.36-7.22(\mathrm{~m}, 5 \mathrm{H}), 7.08(\mathrm{t}, J$ $=8.0 \mathrm{~Hz}, 1 \mathrm{H}), 6.62-6.55(\mathrm{~m}, 2 \mathrm{H}), 6.33(\mathrm{ddd}, J=8.1,2.2,0.9 \mathrm{~Hz}, 1 \mathrm{H}), 4.35(\mathrm{br} \mathrm{s}, 1 \mathrm{H}), 4.30(\mathrm{t}, J=$ $6.3 \mathrm{~Hz}, 1 \mathrm{H}), 3.13(\mathrm{~d}, J=6.3 \mathrm{~Hz}, 2 \mathrm{H}), 1.56(\mathrm{~s}, 9 \mathrm{H}), 1.39(\mathrm{~s}, 9 \mathrm{H}) .{ }^{13} \mathrm{C}$ NMR $\left(100 \mathrm{MHz}, \mathrm{CDCl}_{3}\right) \delta$ $172.2,152.7,147.3,139.5,136.6,129.7,129.6,128.3,126.8,108.3,108.2,103.6,81.8,80.2,58.0$, 38.6, 28.4, 27.9. IR (neat, $\mathrm{cm}^{-1}$ ): 3317, 2976, 1691, 1596, 1533, 1150. Anal. Calcd. for $\mathrm{C}_{24} \mathrm{H}_{32} \mathrm{~N}_{2} \mathrm{O}_{4}$ : C, 69.88; H, 7.82, Found: C, 70.00; H, 7.86. $[\alpha]_{\mathrm{D}}{ }^{24}+11.7$ (c 1.0, $\left.\mathrm{CHCl}_{3}\right)$. HPLC analysis (AD-H, 10\% IPA-hexanes, $0.8 \mathrm{~mL} / \mathrm{min}, 254 \mathrm{~nm}$ ) indicated $80 \%$ ee: tR (major) $=12.8$ $\min , \mathrm{tR}($ minor $)=15.0 \mathrm{~min}$.

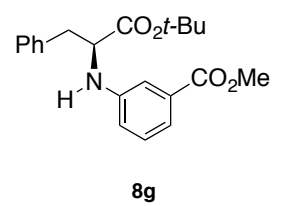

Following the general procedure, a mixture of L-Phe-Ot-Bu (5a, $221 \mathrm{mg}, 1.00 \mathrm{mmol}, 1.00$ equiv), methyl 3-trifluoromethanesulfonyloxy-benzoate (7g, $284 \mathrm{mg}, 1.00 \mathrm{mmol}, 1$ equiv), $\mathbf{P 3}$ (43.0 mg, $50.0 \mu \mathrm{mol}, 5.0 \mathrm{~mol} \%)$, cesium carbonate $(977 \mathrm{mg}, 3.00 \mathrm{mmol}, 3.00$ equiv), and 2methyltetrahydrofuran $(2.00 \mathrm{~mL})$ was stirred at $50{ }^{\circ} \mathrm{C}$ for $2 \mathrm{~h}$. The crude product was purified by automated flash-column chromatography (eluting with $2 \%$ EtOAc-hexanes initially, grading to $20 \%$ EtOAc-hexanes, linear gradient) to afford $\mathbf{8 g}$ as a yellow oil. Yield: $343 \mathrm{mg}, 97 \% .{ }^{1} \mathrm{H}$ NMR $\left(400 \mathrm{MHz}, \mathrm{CDCl}_{3}\right) \delta 7.43(\mathrm{ddd}, J=7.6,1.6,1.0 \mathrm{~Hz}, 1 \mathrm{H}), 7.36-7.21(\mathrm{~m}, 7 \mathrm{H}), 6.81$ (ddd, $J=8.1$, 2.6, 1.0 Hz, 1H), $4.42(\mathrm{br} \mathrm{s}, 1 \mathrm{H}), 4.32(\mathrm{t}, J=6.4 \mathrm{~Hz}, 1 \mathrm{H}), 3.91(\mathrm{~s}, 3 \mathrm{H}), 3.15(\mathrm{~d}, J=6.4 \mathrm{~Hz}, 2 \mathrm{H})$, 1.39 (s, 9H). ${ }^{13} \mathrm{C}$ NMR $\left(100 \mathrm{MHz}, \mathrm{CDCl}_{3}\right) \delta 172.1,167.4,146.7,136.5,131.2,129.6,129.3$, 128.5, 127.0, 119.4, 118.3, 114.0, 82.1, 58.1, 52.1, 38.6, 28.0. IR (neat, $\mathrm{cm}^{-1}$ ): 3367, 2978, 1717 , 1245, 1149, 752. HRMS. Calcd. for $\mathrm{C}_{21} \mathrm{H}_{26} \mathrm{NO}_{4},[\mathrm{M}+\mathrm{H}]: 356.1856$, Found: $[\mathrm{M}+\mathrm{H}]: 356.1845$ $[\alpha]_{\mathrm{D}}{ }^{24}+6.4\left(c 1.0, \mathrm{CHCl}_{3}\right)$. HPLC analysis (OJ-H, 10\% IPA-hexanes, $\left.1.0 \mathrm{~mL} / \mathrm{min}, 254 \mathrm{~nm}\right)$ indicated $82 \%$ ee: $\mathrm{tR}$ (major) $=11.9 \mathrm{~min}, \mathrm{tR}($ minor $)=18.8 \mathrm{~min}$.

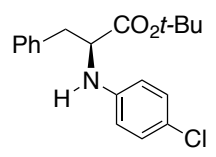

$8 \mathrm{~h}$

Following the general procedure, a mixture of L-Phe-Ot-Bu (5a, $221 \mathrm{mg}, 1.00 \mathrm{mmol}, 1.00$ equiv), 4-chlorophenyl trifluoromethanesulfonate $(7 \mathbf{h}, 261 \mathrm{mg}, 1.00 \mathrm{mmol}, 1$ equiv), $\mathbf{P 3}$ (43.0 mg, 50.0 $\mu \mathrm{mol}, \quad 5.0 \mathrm{~mol} \%)$, cesium carbonate $(977 \mathrm{mg}, 3.00 \mathrm{mmol}, 3.00$ equiv), and 2- 
methyltetrahydrofuran $(2.00 \mathrm{~mL})$ was stirred at $50{ }^{\circ} \mathrm{C}$ for $2 \mathrm{~h}$. The crude product was purified by automated flash-column chromatography (eluting with $2 \%$ ether-hexanes initially, grading to $20 \%$ ether-hexanes, linear gradient) to afford $\mathbf{8 h}$ as a white solid. Yield: $307 \mathrm{mg}, 93 \% . \mathrm{mp}=75-$ $78{ }^{\circ} \mathrm{C} .{ }^{1} \mathrm{H}$ NMR $\left(400 \mathrm{MHz}, \mathrm{CDCl}_{3}\right) \delta$ 7.40-7.26 (m, 5H), 7.21-7.15 (m, 2H), 6.62-6.56 (m, 2H), 4.37 (br s, 1H), 4.28 (t, $J=6.4 \mathrm{~Hz}, 1 \mathrm{H}), 3.16(\mathrm{~d}, J=6.3 \mathrm{~Hz}, 2 \mathrm{H}), 1.44(\mathrm{~s}, 9 \mathrm{H}) .{ }^{13} \mathrm{C}$ NMR $(100$ $\left.\mathrm{MHz}, \mathrm{CDCl}_{3}\right) \delta 172.0,145.3,136.4,129.5,129.1,128.4,126.9,122.6,114.7,81.9,58.2,38.5$, 27.9. IR (neat, $\mathrm{cm}^{-1}$ ): $3359,2985,1709,1600,817,698$. Anal. Calcd. for $\mathrm{C}_{19} \mathrm{H}_{22} \mathrm{ClNO}_{2}$ : $\mathrm{C}, 68.77$; $\mathrm{H}, 6.68$, Found: $\mathrm{C}, 68.88 ; \mathrm{H}, 6.79$. $[\alpha]_{\mathrm{D}}{ }^{24}+25.8\left(c 1.0, \mathrm{CHCl}_{3}\right)$. HPLC analysis $(\mathrm{OJ}-\mathrm{H}, 5 \%$ IPAhexanes, $0.8 \mathrm{~mL} / \mathrm{min}, 254 \mathrm{~nm}$ ) indicated $91 \%$ ee: $\mathrm{tR}$ (minor) $=15.7 \mathrm{~min}$, $\mathrm{tR}$ (major) $=19.8 \mathrm{~min}$.

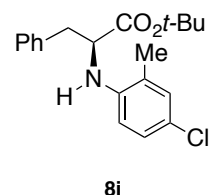

Following the general procedure, a mixture of L-Phe-Ot-Bu (5a, $221 \mathrm{mg}, 1.00 \mathrm{mmol}, 1.00$ equiv), 4-chloro-2-methylphenyl trifluoromethanesulfonate (7i, $275 \mathrm{mg}, 1.00 \mathrm{mmol}, 1$ equiv), P3 (43.0 $\mathrm{mg}, 50.0 \mu \mathrm{mol}, 5.0 \mathrm{~mol} \%$ ), cesium carbonate (977 $\mathrm{mg}, 3.00 \mathrm{mmol}, 3.00$ equiv), and 2methyltetrahydrofuran $(2.00 \mathrm{~mL})$ was stirred at $50{ }^{\circ} \mathrm{C}$ for $2 \mathrm{~h}$. The crude product was purified by automated flash-column chromatography (eluting with $1 \%$ ether-pentane initially, grading to $10 \%$ ether-pentane, linear gradient) to afford $8 \mathbf{i}$ as a yellow oil. Yield: $291 \mathrm{mg}, 84 \% .{ }^{1} \mathrm{H}$ NMR $\left(400 \mathrm{MHz}, \mathrm{CDCl}_{3}\right) \delta$ 7.37-7.26 (m, 3H), 7.26-7.21 (m, 2H), 7.12-7.05 (m, 2H), $6.52(\mathrm{~d}, J=8.2$ $\mathrm{Hz}, 1 \mathrm{H}), 4.29(\mathrm{t}, J=6.2 \mathrm{~Hz}, 1 \mathrm{H}), 4.11(\mathrm{~s}, 1 \mathrm{H}), 3.19(\mathrm{dd}, J=6.2,2.3 \mathrm{~Hz}, 2 \mathrm{H}), 2.12(\mathrm{~s}, 3 \mathrm{H}), 1.42(\mathrm{~s}$, 9H). ${ }^{13} \mathrm{C}$ NMR $\left(100 \mathrm{MHz}, \mathrm{CDCl}_{3}\right) \delta 172.1,143.3,136.5,130.1,129.6,128.5,127.0,126.7,124.6$, 122.2, 111.5, 82.0, 58.0, 38.4, 28.0, 17.3. IR (neat, $\mathrm{cm}^{-1}$ ): 3424, 2977, 1726, 1505, 1147, 690 . Anal. Calcd. for $\mathrm{C}_{20} \mathrm{H}_{24} \mathrm{ClNO}_{2}$ : C, 69.45; H, 6.99, Found: C, 69.71; H, 6.96. [ $\left.\alpha\right]_{\mathrm{D}}{ }^{24}+14.7$ (c 1.0, $\left.\mathrm{CHCl}_{3}\right)$. HPLC analysis (OJ-H, $2 \%$ IPA-hexanes, $\left.0.8 \mathrm{~mL} / \mathrm{min}, 254 \mathrm{~nm}\right)$ indicated $88 \%$ ee: tR $($ minor $)=11.9 \mathrm{~min}, \mathrm{tR}($ major $)=18.0 \mathrm{~min}$.

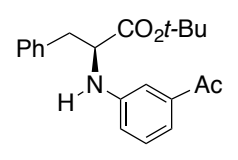

8j

Following the general procedure, a mixture of L-Phe-Ot-Bu (5a, $221 \mathrm{mg}, 1.00 \mathrm{mmol}, 1.00$ equiv), 3-acetylphenyl trifluoromethanesulfonate (7j, $268 \mathrm{mg}, 1.00 \mathrm{mmol}, 1$ equiv), P3 (43.0 mg, 50.0 $\mu \mathrm{mol}, \quad 5.0 \mathrm{~mol} \%)$, cesium carbonate (977 mg, $3.00 \mathrm{mmol}, 3.00$ equiv), and 2methyltetrahydrofuran $(2.00 \mathrm{~mL})$ was stirred at $50{ }^{\circ} \mathrm{C}$ for $2 \mathrm{~h}$. The crude product was purified by automated flash-column chromatography with a new column (eluting with $2 \%$ acetone-hexanes initially, grading to $20 \%$ acetone-hexanes, linear gradient) to afford $\mathbf{8 j}$ as a yellow oil. Yield: $305 \mathrm{mg}, 90 \% .{ }^{1} \mathrm{H}$ NMR $\left(400 \mathrm{MHz}, \mathrm{CDCl}_{3}\right) \delta 7.35-7.29(\mathrm{~m}, 3 \mathrm{H}), 7.29-7.20(\mathrm{~m}, 5 \mathrm{H}), 6.81$ (ddd, $J$ $=7.9,2.6,1.0 \mathrm{~Hz}, 1 \mathrm{H}), 4.43(\mathrm{br} \mathrm{s}, 1 \mathrm{H}), 4.32(\mathrm{t}, J=6.4 \mathrm{~Hz}, 1 \mathrm{H}), 3.14(\mathrm{~d}, J=6.1 \mathrm{~Hz}, 2 \mathrm{H}), 2.56(\mathrm{~s}$, $3 \mathrm{H}), 1.39(\mathrm{~s}, 9 \mathrm{H}) .{ }^{13} \mathrm{C} \mathrm{NMR}\left(100 \mathrm{MHz}, \mathrm{CDCl}_{3}\right) \delta 198.4,172.1,147.0,138.2,136.5,129.5,129.4$, 128.5, 127.0, 118.6, 118.5, 112.2, 82.1, 58.0, 38.6, 28.0, 26.7. IR (neat, $\mathrm{cm}^{-1}$ ): 3361, 2977, 1725, 1679, 1601, 1149, 699. HRMS. Calcd. for $\mathrm{C}_{21} \mathrm{H}_{26} \mathrm{NO}_{3},[\mathrm{M}+\mathrm{H}]: 340.1907$, Found: $[\mathrm{M}+\mathrm{H}]$ : $340.1920[\alpha]_{\mathrm{D}}{ }^{24}+3.1\left(c 1.0, \mathrm{CHCl}_{3}\right)$. HPLC analysis (AD-H, 10\% IPA-hexanes, $1.0 \mathrm{~mL} / \mathrm{min}, 254$ $\mathrm{nm})$ indicated $85 \%$ ee: $\mathrm{tR}($ minor $)=13.9 \mathrm{~min}, \mathrm{tR}($ major $)=20.2 \mathrm{~min}$. 


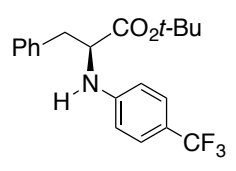

$8 \mathbf{k}$

Following the general procedure, a mixture of L-Phe-Ot-Bu (5a, $221 \mathrm{mg}, 1.00 \mathrm{mmol}, 1.00$ equiv), 4-(trifluoromethyl)phenyl trifluoromethanesulfonate (7k, $294 \mathrm{mg}, 1.00 \mathrm{mmol}, 1$ equiv), $\mathbf{P 3}$ (43.0 $\mathrm{mg}, 50.0 \mu \mathrm{mol}, 5.0 \mathrm{~mol} \%$ ), cesium carbonate (977 $\mathrm{mg}, 3.00 \mathrm{mmol}, 3.00$ equiv), and 2methyltetrahydrofuran $(2.00 \mathrm{~mL})$ was stirred at $50{ }^{\circ} \mathrm{C}$ for $2 \mathrm{~h}$. The crude product was purified by automated flash-column chromatography (eluting with $2 \%$ ether-hexanes initially, grading to $20 \%$ ether-hexanes, linear gradient) to afford $\mathbf{8 k}$ as a white solid. Yield: $283 \mathrm{mg}, 77 \% . \mathrm{mp}=80$ $81{ }^{\circ} \mathrm{C} .{ }^{1} \mathrm{H}$ NMR $\left(400 \mathrm{MHz}, \mathrm{CDCl}_{3}\right) \delta 7.43(\mathrm{~d}, J=8.5 \mathrm{~Hz}, 2 \mathrm{H}), 7.37-7.25(\mathrm{~m}, 3 \mathrm{H}), 7.25-7.19(\mathrm{~m}$, $2 \mathrm{H}), 6.63(\mathrm{~d}, J=8.5 \mathrm{~Hz}, 2 \mathrm{H}), 4.57(\mathrm{br} \mathrm{s}, 1 \mathrm{H}), 4.31(\mathrm{t}, J=6.2 \mathrm{~Hz}, 1 \mathrm{H}), 3.15(\mathrm{dd}, J=6.2,3.4 \mathrm{~Hz}$, $2 \mathrm{H}), 1.41(\mathrm{~s}, 9 \mathrm{H}) .{ }^{13} \mathrm{C} \mathrm{NMR}\left(100 \mathrm{MHz}, \mathrm{CDCl}_{3}\right) \delta 171.7,149.2,136.3,129.6,128.6,127.2,126.8$ $(\mathrm{q}, J=3.0 \mathrm{~Hz}), 125.0(\mathrm{q}, J=270.0 \mathrm{~Hz}), 119.8(\mathrm{q}, J=30.0 \mathrm{~Hz}), 112.7,82.4,57.6,38.5,28.1 .{ }^{19} \mathrm{~F}$ NMR (375 MHz, $\mathrm{CDCl}_{3}$ ) -61.12. IR (neat, $\mathrm{cm}^{-1}$ ): 3382, 2988, 1708, 1616, 1317, 1104. Anal. Calcd. for $\mathrm{C}_{20} \mathrm{H}_{22} \mathrm{~F}_{3} \mathrm{NO}_{2}$ : C, 65.74; H, 6.07, Found: C, 65.89; H, 6.03. [ $\left.\alpha\right]_{\mathrm{D}}{ }^{24}+16.4\left(c 1.0, \mathrm{CHCl}_{3}\right)$. HPLC analysis (OJ-H, 2\% IPA-hexanes, $0.8 \mathrm{~mL} / \mathrm{min}, 254 \mathrm{~nm}$ ) indicated $55 \%$ ee: tR (minor) $=$ $18.4 \mathrm{~min}$, tR (major) $=24.4 \mathrm{~min}$.

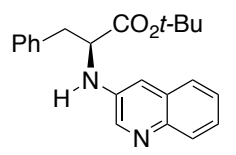

81

Following the general procedure, a mixture of L-Phe-Ot-Bu (5a, $221 \mathrm{mg}, 1.00 \mathrm{mmol}, 1.00$ equiv), 3-quinolinyl trifluoromethanesulfonate (7l, $277 \mathrm{mg}, 1.00 \mathrm{mmol}, 1$ equiv), P3 (43.0 mg, $50.0 \mu \mathrm{mol}$, $5.0 \mathrm{~mol} \%)$, cesium carbonate $(977 \mathrm{mg}, 3.00 \mathrm{mmol}, 3.00$ equiv), and 2-methyltetrahydrofuran $(2.00 \mathrm{~mL})$ was stirred at $50{ }^{\circ} \mathrm{C}$ for $2 \mathrm{~h}$. The crude product was purified by automated flashcolumn chromatography (eluting with $12 \%$ EtOAc-hexanes initially, grading to $100 \%$ EtOAchexanes, linear gradient) to afford $\mathbf{8 1}$ as an orange oil. Yield: $241 \mathrm{mg}, 69 \% .{ }^{1} \mathrm{H}$ NMR $(400 \mathrm{MHz}$, $\left.\mathrm{CDCl}_{3}\right) \delta 8.48(\mathrm{~d}, J=2.8 \mathrm{~Hz}, 1 \mathrm{H}), 8.01-7.94(\mathrm{~m}, 1 \mathrm{H}), 7.63-7.56(\mathrm{~m}, 1 \mathrm{H}), 7.44(\mathrm{dt}, J=6.0,3.6$ $\mathrm{Hz}, 2 \mathrm{H}), 7.36-7.23(\mathrm{~m}, 5 \mathrm{H}), 7.03(\mathrm{~d}, J=2.8 \mathrm{~Hz}, 1 \mathrm{H}), 4.69(\mathrm{~d}, J=8.3 \mathrm{~Hz}, 1 \mathrm{H}), 4.37(\mathrm{dt}, J=8.3$, $6.3 \mathrm{~Hz}, 1 \mathrm{H}), 3.29-3.14(\mathrm{~m}, 2 \mathrm{H}), 1.41(\mathrm{~s}, 9 \mathrm{H}) .{ }^{13} \mathrm{C} \mathrm{NMR}\left(100 \mathrm{MHz}, \mathrm{CDCl}_{3}\right) \delta 171.7,143.5,142.4$, $140.1,136.3,129.5,129.3,129.1,128.5,127.1,127.0,126.0,125.3,111.4,82.4,57.9,38.2,28.0$. IR (neat, $\mathrm{cm}^{-1}$ ): 3365, 2977, 1725, 1608, 1148, 732. HRMS. Calcd. for $\mathrm{C}_{22} \mathrm{H}_{25} \mathrm{~N}_{2} \mathrm{O}_{2}$, $[\mathrm{M}+\mathrm{H}]$ : 349.1911, Found: $[\mathrm{M}+\mathrm{H}]: 349.1926[\alpha]_{\mathrm{D}}^{24}+18.0\left(c 1.0, \mathrm{CHCl}_{3}\right) . \quad$ HPLC analysis $(\mathrm{AD}-\mathrm{H}, 10 \%$ IPA-hexanes, $1.0 \mathrm{~mL} / \mathrm{min}, 254 \mathrm{~nm}$ ) indicated $97 \%$ ee: $\mathrm{tR}($ minor $)=12.3 \mathrm{~min}$, $\mathrm{tR}$ (major) $=25.7$ $\min$.

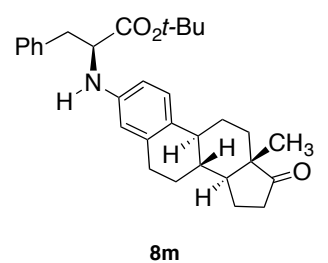

Following the general procedure, a mixture of L-Phe-Ot-Bu (5a, $221 \mathrm{mg}, 1.00 \mathrm{mmol}, 1.00$ equiv), estrone trifluoromethanesulfonate $(\mathbf{7 m}, 402 \mathrm{mg}, 1.00 \mathrm{mmol}, 1$ equiv), P1 (43.0 mg, $50.0 \mu \mathrm{mol}$, $5.0 \mathrm{~mol} \%$ ), cesium carbonate ( $977 \mathrm{mg}, 3.00 \mathrm{mmol}, 3.00$ equiv), and 2-methyltetrahydrofuran $(2.00 \mathrm{~mL})$ was stirred at $50{ }^{\circ} \mathrm{C}$ for $2 \mathrm{~h}$. The crude product was purified by automated flashcolumn chromatography (eluting with $8 \%$ ether-hexanes initially, grading to $66 \%$ ether-hexanes, linear gradient) to afford $\mathbf{8 m}$ as a clear oil. Yield: $463 \mathrm{mg}, 98 \% .{ }^{1} \mathrm{H}$ NMR $\left(400 \mathrm{MHz}, \mathrm{CDCl}_{3}\right) \delta$ 
7.39-7.24 (m, 5H), 7.15-7.04 (m, 1H), $6.49(\mathrm{dd}, J=8.4,2.5 \mathrm{~Hz}, 1 \mathrm{H}), 6.41(\mathrm{~d}, J=2.4 \mathrm{~Hz}, 1 \mathrm{H})$, 4.26 (app t, $J=6.5 \mathrm{~Hz}, 2 \mathrm{H}), 3.13(\mathrm{~d}, J=6.4 \mathrm{~Hz}, 2 \mathrm{H}), 2.99-2.80(\mathrm{~m}, 2 \mathrm{H}), 2.52(\mathrm{dd}, J=18.8,8.8$ $\mathrm{Hz}, 1 \mathrm{H}), 2.45-2.34(\mathrm{~m}, 1 \mathrm{H}), 2.28-1.95(\mathrm{~m}, 5 \mathrm{H}), 1.74-1.46(\mathrm{~m}, 6 \mathrm{H}), 1.42$ (s, 9H), 0.94 (app d, $J=$ $6.0 \mathrm{~Hz}, 3 \mathrm{H}) .{ }^{13} \mathrm{C}$ NMR $\left(100 \mathrm{MHz}, \mathrm{CDCl}_{3},{ }^{*}\right.$ denotes rotamer, when observed ) $\delta 220.6,219.9 *$, $172.2,147.4^{*}, 144.5,140.2^{*}, 139.2^{*}, 137.0,136.7,129.4(2 \mathrm{C}), 128.1,127.1 *, 126.6,126.0$, $121.0^{*}, 120.2^{*}, 118.1^{*}, 117.0^{*}, 113.4,111.4,81.3,58.1,50.2,50.1^{*}, 47.8,47.6^{*}, 43.9^{*}, 43.8$, $38.7,38.3,37.5^{*}, 36.5^{*}, 35.7,35.6^{*}, 31.5,31.3^{*}, 29.5,29.2^{*}, 27.8,26.5,25.9^{*}, 25.8,25.5^{*}, 21.4$, 13.7, 13.6*. IR (neat, $\mathrm{cm}^{-1}$ ): 3378, 2928, 1733, 1615, 1149, 732, 700. HRMS. Calcd. for $\mathrm{C}_{31} \mathrm{H}_{40} \mathrm{NO}_{3},[\mathrm{M}+\mathrm{H}]: 474.3003$, Found: $[\mathrm{M}+\mathrm{H}]: 474.3016[\alpha]_{\mathrm{D}}{ }^{24}+77.7$ (c 1.0, $\mathrm{CHCl}_{3}$ ). The product $9 \mathrm{~m}$ prepared in this way was 19.6:1.0 d.r. [as determined by inverse-gated ${ }^{13} \mathrm{C} N M R$ $($ relaxation delay $=20 \mathrm{~s})]$. 
B) Preparation of Starting Materials
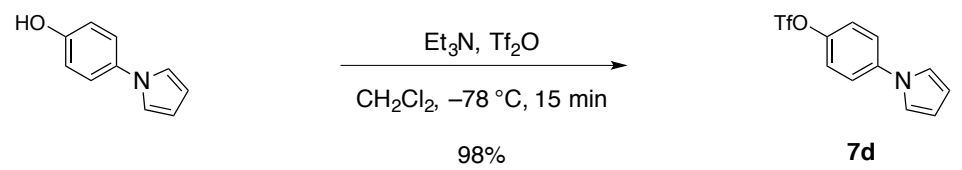

Preparation of 4-(1H-pyrrol-1-yl)phenyl trifluoromethanesulfonate (7d): Triethylamine $(607 \mathrm{mg}$, $6.00 \mathrm{mmol}, 1.20$ equiv) and trifluoromethanesulfonic anhydride (1.55 g, $5.50 \mathrm{mmol}, 1.10$ equiv) were added in sequence to a solution of $4-(1 H$-pyrrol-1-yl)phenol $(796 \mathrm{mg}, 5.00 \mathrm{mmol}, 1$ equiv) in $\mathrm{CH}_{2} \mathrm{Cl}_{2}(30 \mathrm{~mL})$ at $-78{ }^{\circ} \mathrm{C}$. The reaction mixture was stirred for $15 \mathrm{~min}$ at $-78{ }^{\circ} \mathrm{C}$. After warming to room temperature, the product mixture was transferred to a separatory funnel that had been charged with $\mathrm{CH}_{2} \mathrm{Cl}_{2}(20 \mathrm{~mL})$. The diluted product mixture was washed with saturated aqueous sodium bicarbonate solution $(50 \mathrm{~mL})$. The aqueous layer was isolated and the isolated aqueous layer was extracted with $\mathrm{CH}_{2} \mathrm{Cl}_{2}(3 \times 50 \mathrm{~mL})$. The organic layers were combined and the combined organic layers were dried over sodium sulfate. The dried solution was filtered and the filtrate was concentrated. The residue obtained was purified by automated flash-column chromatography (eluting with $2 \%$ EtOAc-hexanes initially, grading to $20 \%$ EtOAc-hexanes, linear gradient) to afford 4-(1H-pyrrol-1-yl)phenyl trifluoromethanesulfonate (7d) as a white solid. Yield: $1.43 \mathrm{~g}, 98 \% . \mathrm{mp}=49-51{ }^{\circ} \mathrm{C} .{ }^{1} \mathrm{H}$ NMR $\left(400 \mathrm{MHz}, \mathrm{CDCl}_{3}\right) \delta 7.48-7.42(\mathrm{~m}, 2 \mathrm{H})$, $7.39-7.33(\mathrm{~m}, 2 \mathrm{H}), 7.09(\mathrm{t}, J=2.2 \mathrm{~Hz}, 2 \mathrm{H}), 6.42(\mathrm{t}, J=2.2 \mathrm{~Hz}, 2 \mathrm{H}) .{ }^{13} \mathrm{C}$ NMR $(100 \mathrm{MHz}$, $\left.\mathrm{CDCl}_{3}\right) \delta 146.7,140.6,122.7,121.6,119.4,118.9(\mathrm{q}, J=320.0 \mathrm{~Hz}), 111.5 .{ }^{19} \mathrm{~F}$ NMR $(375 \mathrm{MHz}$, $\left.\mathrm{CDCl}_{3}\right) \delta$-72.74. IR (neat, $\mathrm{cm}^{-1}$ ): 1515, 1426, 1207, 1133, 881, 835, 723, 605. Anal. Calcd. for $\mathrm{C}_{11} \mathrm{H}_{8} \mathrm{~F}_{3} \mathrm{NO}_{3} \mathrm{~S}: \mathrm{C}, 45.36$; H, 2.77, Found: $\mathrm{C}, 45.53 ; \mathrm{H}, 2.86$.
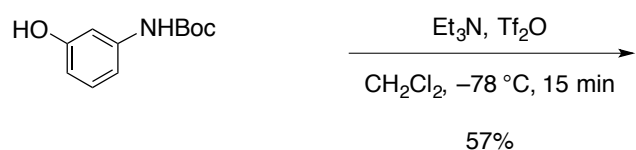

$57 \%$

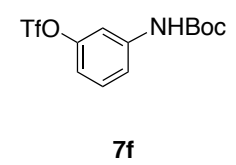
Preparation of 3-((tert-butoxycarbonyl)amino)phenyl trifluoromethanesulfonate (7f):
Triethylamine (607 mg, $6.00 \mathrm{mmol}, 1.20$ equiv) and trifluoromethanesulfonic anhydride (1.55 g, $5.50 \mathrm{mmol}, 1.10$ equiv) were added in sequence to a solution of $N$-Boc-3-aminophenol $(1.05 \mathrm{~g}$, $5.00 \mathrm{mmol}, 1$ equiv) in $\mathrm{CH}_{2} \mathrm{Cl}_{2}(30 \mathrm{~mL})$ at $-78^{\circ} \mathrm{C}$. The reaction mixture was stirred for $15 \mathrm{~min}$ at $-78{ }^{\circ} \mathrm{C}$. After warming to room temperature, the product mixture was transferred to a separatory funnel that had been charged with $\mathrm{CH}_{2} \mathrm{Cl}_{2}(20 \mathrm{~mL})$. The diluted product mixture was washed with saturated aqueous sodium bicarbonate solution $(50 \mathrm{~mL})$. The aqueous layer was isolated and the isolated aqueous layer was extracted with $\mathrm{CH}_{2} \mathrm{Cl}_{2}(3 \times 50 \mathrm{~mL})$. The organic layers were combined and the combined organic layers were dried over sodium sulfate. The dried solution was filtered and the filtrate was concentrated. The residue obtained was purified by automated flash-column chromatography (eluting with 5\% EtOAc-hexanes initially, grading to $40 \%$ EtOAc-hexanes, linear gradient) to afford 3-((tert-butoxycarbonyl)amino)phenyl trifluoromethanesulfonate (7f) as a white solid. Yield: $1.16 \mathrm{~g}, 57 \% . \mathrm{mp}=74-76{ }^{\circ} \mathrm{C} .{ }^{1} \mathrm{H}$ NMR $\left(400 \mathrm{MHz}, \mathrm{CDCl}_{3}\right) \delta 7.57(\mathrm{~s}, 1 \mathrm{H}), 7.32(\mathrm{t}, J=8.2 \mathrm{~Hz}, 1 \mathrm{H}), 7.25(\mathrm{~d}, J=8.3 \mathrm{~Hz}, 1 \mathrm{H}), 7.01-6.90(\mathrm{~m}$, $2 \mathrm{H}), 1.54(\mathrm{~s}, 9 \mathrm{H}) .{ }^{13} \mathrm{C}$ NMR $\left(100 \mathrm{MHz}, \mathrm{CDCl}_{3}\right) \delta 152.5,150.0,140.6,130.4,118.8(\mathrm{q}, \mathrm{J}=320.0$ $\mathrm{Hz}), 118.0,115.3,111.5,81.5,28.3 .{ }^{19} \mathrm{~F}$ NMR $\left(375 \mathrm{MHz}, \mathrm{CDCl}_{3}\right)$-73.04. IR (neat, $\left.\mathrm{cm}^{-1}\right)$ : 3326, 2976, 1693, 1533, 1417, 1288, 1206, 1138. Anal. Calcd. for $\mathrm{C}_{12} \mathrm{H}_{14} \mathrm{~F}_{3} \mathrm{NO}_{5} \mathrm{~S}$ : C, 42.23; H, 4.13, Found: C, 42.49; H, 4.09 . 

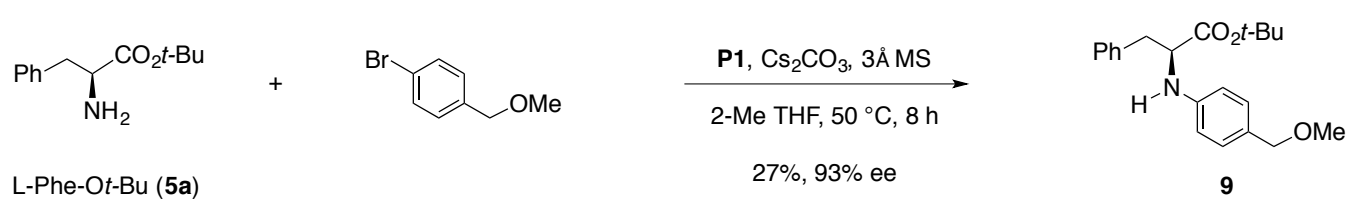

Preparation of the N-arylation product 9: A $25 \mathrm{~mL}$ screw-cap tube equipped with a stir bar and Teflon septum was charged sequentially with L-Phe-Ot-Bu, (5a, $443 \mathrm{mg}, 2.00 \mathrm{mmol}, 1.00$ equiv), 1-bromo-4-(methoxymethyl)benzene, (402 mg, $2.00 \mathrm{mmol}, 1.00$ equiv), P1 (85.4 mg, $100 \mu \mathrm{mol}$, $5.0 \mathrm{~mol} \%)$, and cesium carbonate $(1.95 \mathrm{~g}, 6.00 \mathrm{mmol}, 3.00$ equiv). The reaction test tube was capped and then evacuated and backfilled with argon by piercing with a needle attached to a Schlenk line (this process was repeated a total of three times). 2-Methyltetrahydrofuran $(4.00 \mathrm{~mL})$ was added to the reaction test tube. The reaction test tube was placed in an oil bath that had been preheated to $50{ }^{\circ} \mathrm{C}$. The reaction mixture was stirred and heated at $50{ }^{\circ} \mathrm{C}$ for $8 \mathrm{~h}$. The reaction mixture was allowed to cool over $20 \mathrm{~min}$ to $\mathrm{rt}$. The cooled product mixture was diluted with $\mathrm{CH}_{2} \mathrm{Cl}_{2}(5.00 \mathrm{~mL})$. The diluted product mixture was filtered through Celite and concentrated. The residue obtained was purified by automated flash-column chromatography (eluting with $8 \%$ ether-hexanes initially, grading to $66 \%$ ether-hexanes, linear gradient) to afford 9 as a yellow oil. Yield: $186 \mathrm{mg}, 27 \%$. ${ }^{1} \mathrm{H}$ NMR $\left(400 \mathrm{MHz}, \mathrm{CDCl}_{3}\right) \delta 7.22-7.09(\mathrm{~m}, 5 \mathrm{H}), 7.08-7.02(\mathrm{~m}, 2 \mathrm{H}), 6.53-$ $6.47(\mathrm{~m}, 2 \mathrm{H}), 4.24(\mathrm{~s}, 2 \mathrm{H}), 4.20-4.11(\mathrm{~m}, 1 \mathrm{H}), 3.24(\mathrm{~s}, 3 \mathrm{H}), 3.00(\mathrm{~d}, J=6.0 \mathrm{~Hz}, 2 \mathrm{H}), 1.25(\mathrm{~s}, 9 \mathrm{H})$. ${ }^{13} \mathrm{C}$ NMR $\left(100 \mathrm{MHz}, \mathrm{CDCl}_{3}\right) \delta 172.2,146.3,136.7,129.6$ (2 C), 128.4, 127.7, 126.9, 113.5, 81.8, 74.7, 58.2, 57.7, 38.6, 28.0. IR (neat, $\mathrm{cm}^{-1}$ ): 3365, 2977, 1726, 1615, 1521, 1367, 1149, 1088, 699 . Anal. Calcd. for $\mathrm{C}_{21} \mathrm{H}_{27} \mathrm{NO}_{3}$ : C, 73.87; H, 7.97, Found: C, 74.04; H, 7.88. $[\alpha]_{\mathrm{D}}{ }^{24}+24.4(c 1.0$, $\mathrm{CHCl}_{3}$ ). HPLC analysis (OD-H, 10\% IPA-hexanes, $0.8 \mathrm{~mL} / \mathrm{min}, 254 \mathrm{~nm}$ ) indicated $93 \%$ ee: $\mathrm{tR}$ $($ minor $)=7.8 \mathrm{~min}, \mathrm{tR}($ major $)=10.6 \mathrm{~min}$.

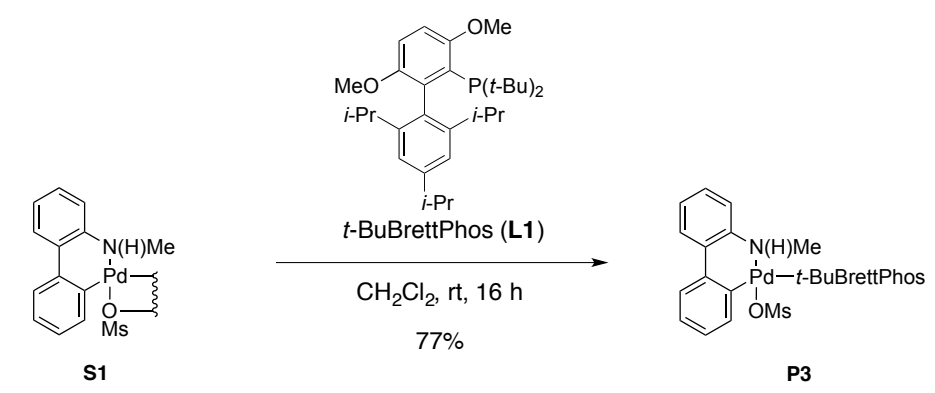

Preparation of $\boldsymbol{P} 3$ [Prepared from a modified literature procedure] ${ }^{1}$ : A $25 \mathrm{~mL}$ screw-cap tube equipped with a stir bar and Teflon septum was charged sequentially with $N$-methyl-2aminobiphenylpalladium methanesulfonate dimer (S1) $(384 \mathrm{mg}, 0.50 \mathrm{mmol}, 0.50$ equiv), $t$ BuBrettPhos (L1) (485 mg, $1.00 \mathrm{mmol}, 1.00$ equiv), and $\mathrm{CH}_{2} \mathrm{Cl}_{2}(5.00 \mathrm{~mL})$. The reaction mixture was stirred at $\mathrm{rt}$ for $16 \mathrm{~h}$. The product mixture was concentrated, and pentane $(25 \mathrm{~mL})$ was added to precipitate the precatalyst, which was isolated via vacuum filtration and dried under vacuum overnight to provide P3 as a yellow solid. Yield: $665 \mathrm{mg}, 77 \%$. ${ }^{1} \mathrm{H} \mathrm{NMR}(400 \mathrm{MHz}$, $\left.\mathrm{CD}_{3} \mathrm{OD}\right)$ Complex Spectrum - See Attached. ${ }^{13} \mathrm{C}$ NMR (125 MHz, $\left.\mathrm{CD}_{3} \mathrm{OD}\right) \delta 161.48,160.98$, $159.09,155.70,155.69,153.00,152.88,151.93,148.77,146.26,143.27,141.87,141.85,140.60$, $137.66,137.62$, 136.22, 129.91, 129.17, 128.94, 128.75, 128.72, 127.96, 126.83, 125.93, 125.36, $124.65,122.73,122.56,122.54,121.02,119.94,116.59,116.58,113.57,113.54,112.79,56.72$, $55.62,55.29,54.70,41.02,41.01,40.72,40.59,40.55,40.42,39.50,35.82,35.48,34.24,33.05$, $33.00,32.59,32.55,32.44,32.31,31.72,29.61,29.59,26.51,26.11,25.87,25.36,24.83,24.74$, 24.38, 24.19, 23.51 (observed complexity due to C-P splitting). ${ }^{31} \mathrm{P}$ NMR (162 MHz, $\left.\mathrm{CD}_{3} \mathrm{OD}\right) \delta$ 79.03, 42.26, 41.84, 41.42, 32.61. IR (neat, $\mathrm{cm}^{-1}$ ): 3222, 2960, 1575, 1456, 1421, 1250, 1144, 1037, 1017, 764, 740 . 
C) Reaction Optimization

Table S1. Summary of initial $N$-arylation experiments.

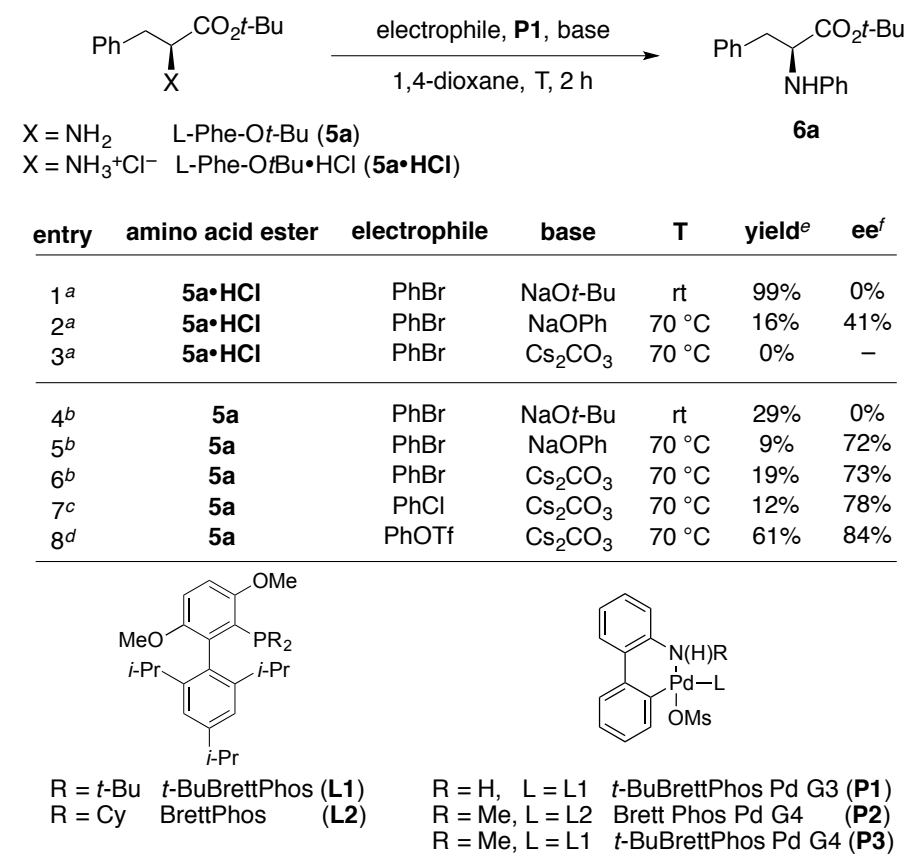

${ }^{a}$ Reaction Conditions: L-Phe-Ot-Bu $\bullet \mathrm{HCl}(\mathbf{5 a} \cdot \mathbf{H C l}, 1.2$ equiv), base (2.4 equiv), bromobenzene (1 equiv), P1 (1 mol\%). ${ }^{b}$ Reaction Conditions: L-Phe-Ot-Bu (5a, 1.2 equiv), base (1.2 equiv), bromobenzene (1 equiv), P1 (1 mol\%). ${ }^{c}$ Reaction Conditions: L-Phe-Ot-Bu (5a, 1.2 equiv), base (1.2 equiv), chlorobenzene (1 equiv), P1 (1 mol\%). ${ }^{d}$ Reaction Conditions: L-Phe-Ot-Bu (5a, 1.2 equiv), base (1.2 equiv), phenyl trifluoromethanesulfonate (1 equiv), P1 (1 mol\%). ${ }^{e}$ Isolated yields. ${ }^{f}$ Enantiomeric excess (ee) was determined by HPLC analysis using chiral stationary phases.

Procedure for entries 1-3: A $10 \mathrm{~mL}$ screw-cap tube equipped with a stir bar and Teflon septum was charged sequentially with L-Phe-O $t$ - $\mathrm{Bu} \bullet \mathbf{H C l}(\mathbf{5 a} \bullet \mathbf{H C l}, 155 \mathrm{mg}, 600 \mu \mathrm{mol}, 1.20$ equiv) and P1 (4.3 mg, $5.0 \mu \mathrm{mol}, 1.0 \mathrm{~mol} \%$ ). The reaction tube was transferred into a nitrogen-filled drybox. Base [sodium tert-butoxide (115 mg, $1.20 \mathrm{~mol}, 2.40$ equiv), sodium phenoxide (139 $\mathrm{mg}, 1.20$ mmol, 2.40 equiv) or cesium carbonate (391 $\mathrm{mg}, 1.20 \mathrm{mmol}, 2.40$ equiv)] was added and the reaction tube was sealed. The sealed reaction tube was removed from the drybox. The reaction test tube was evacuated and backfilled with argon by piercing with a needle attached to a Schlenk line (this process was repeated a total of three times). Bromobenzene $(52.7 \mu \mathrm{L}, 500 \mu \mathrm{mol}, 1.00$ equiv) and 1,4-dioxane $(1.0 \mathrm{~mL})$ were added sequentially to the reaction tube. The reaction mixture was stirred at $\mathrm{rt}$ for $2 \mathrm{~h}$ (entry 1 ) or stirred and heated at $70{ }^{\circ} \mathrm{C}$ for $2 \mathrm{~h}$ (entries 2 and 3 ). The reaction mixture was allowed to cool over $20 \mathrm{~min}$ to $\mathrm{rt}$. The cooled product mixture was diluted with $\mathrm{CH}_{2} \mathrm{Cl}_{2}(3.00 \mathrm{~mL})$. The diluted product mixture was filtered through Celite and concentrated to dryness. The crude product was purified by automated flash-column chromatography (eluting with 2\% EtOAc-hexanes initially, grading to 20\% EtOAc-hexanes, linear gradient) to afford $\mathbf{6 a}$ as a white solid.

Procedure for entries 4-8: A $10 \mathrm{~mL}$ screw-cap tube equipped with a stir bar and Teflon septum was charged sequentially with L-Phe-Ot-Bu (5a, $53.1 \mathrm{mg}, 240 \mu \mathrm{mol}, 1.20$ equiv) and P1 (1.7 mg, $2.0 \mu \mathrm{mol}, 1.0 \mathrm{~mol} \%)$. The reaction tube was transferred into a nitrogen-filled drybox. Base [sodium tert-butoxide $(23.1 \mathrm{mg}, 240 \mu \mathrm{mol}, 1.20$ equiv), sodium phenoxide $(27.9 \mathrm{mg}, 240 \mu \mathrm{mol}$, 1.20 equiv) or cesium carbonate $(78.2 \mathrm{mg}, 240 \mu \mathrm{mol}, 1.20$ equiv)] was added and the reaction tube was sealed. The sealed reaction tube was removed from the drybox. The reaction test tube 
was evacuated and backfilled with argon by piercing with a needle attached to a Schlenk line (this process was repeated a total of three times). Electrophile [bromobenzene $(21.1 \mu \mathrm{L}, 200 \mu \mathrm{mol}$, 1.00 equiv), chlorobenzene ( $20.4 \mu \mathrm{L}, 200 \mu \mathrm{mol}, 1.00$ equiv), or phenyl trifluoromethane sulfonate (32.4 $\mu \mathrm{L}, 200 \mu \mathrm{mol}, 1.00$ equiv)] and 1,4-dioxane $(400 \mu \mathrm{L})$ were added sequentially to the reaction tube. The reaction mixture was stirred at $\mathrm{rt}$ for $2 \mathrm{~h}$ (entry 4 ) or stirred and heated at $70{ }^{\circ} \mathrm{C}$ for $2 \mathrm{~h}$ (entries 5-8). The reaction mixture was allowed to cool over $20 \mathrm{~min}$ to $\mathrm{rt}$. The cooled product mixture was diluted with $\mathrm{CH}_{2} \mathrm{Cl}_{2}(3.00 \mathrm{~mL})$. The diluted product mixture was filtered through Celite and concentrated to dryness. The crude product was purified by automated flash-column chromatography (eluting with $2 \%$ EtOAc-hexanes initially, grading to $20 \%$ EtOAc-hexanes, linear gradient) to afford $\mathbf{6 a}$ as a white solid. 


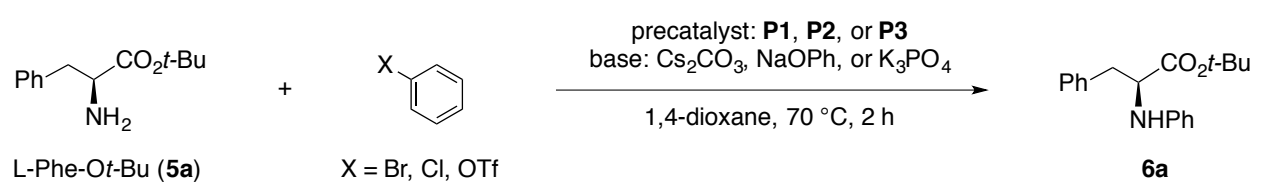

Figure S1. Evaluation of base, electrophile, and precatalyst.

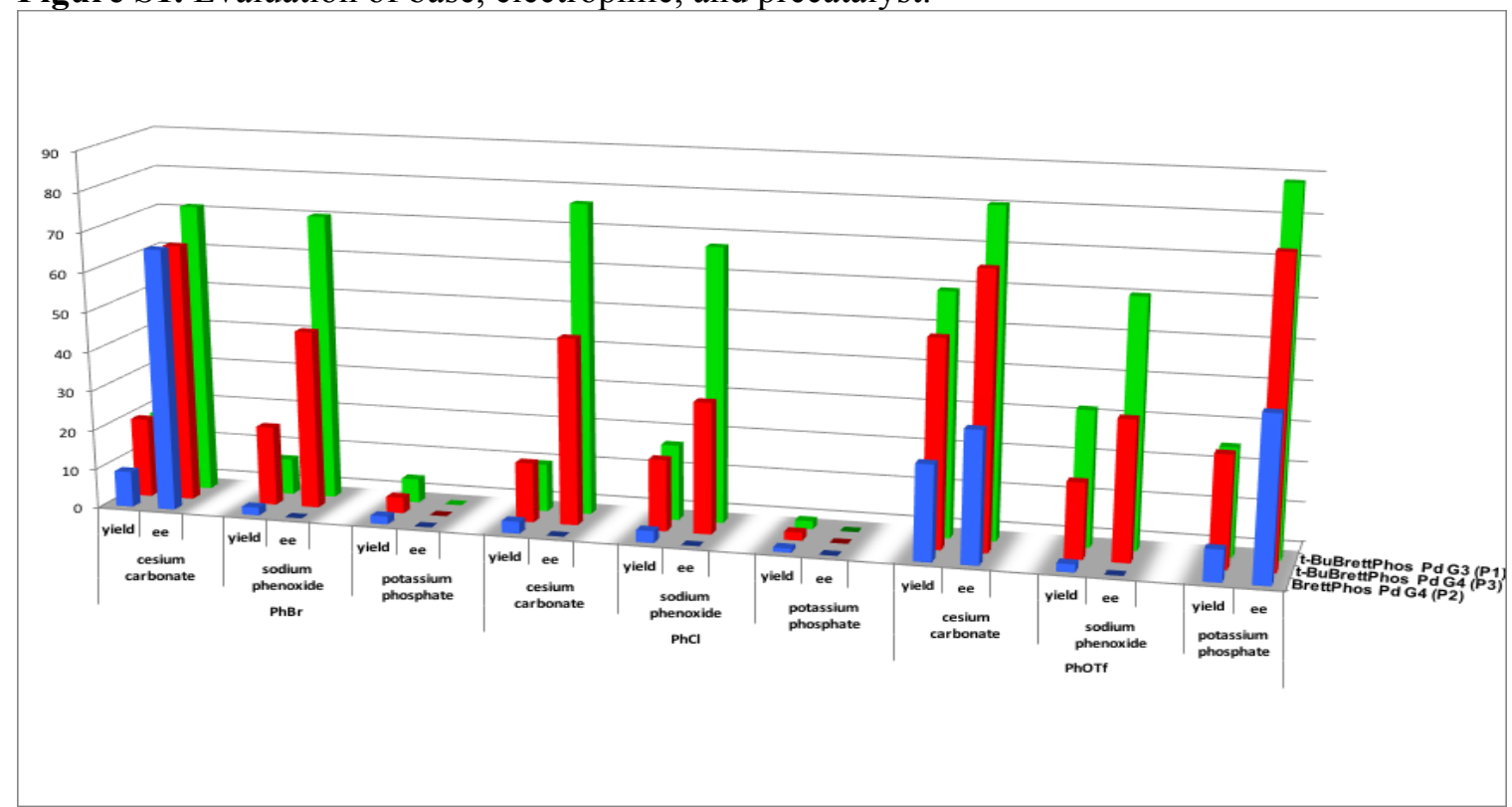

$\mathrm{Z}$ axis: \% yield and \% ee for each reaction. $\mathrm{Y}$ axis: Green $=$ reactions with precatalyst $\mathbf{P 1}$. Red $=$ reactions with precatalyst P3. Blue $=$ reactions with precatalyst P2. X axis: electrophile and base for each reaction. Optimal combination: cesium carbonate, phenyl trifluoromethanesulfonate, and precatalyst P1 (61\% yield, $84 \%$ ee).

Procedure for Figure S1: A $10 \mathrm{~mL}$ screw-cap tube equipped with a stir bar and Teflon septum was charged sequentially with L-Phe-O $t$-Bu (5a, $53.1 \mathrm{mg}, 240 \mu \mathrm{mol}, 1.20$ equiv) and precatalyst [P1 $(1.7 \mathrm{mg}, 2.0 \mu \mathrm{mol}, 1.0 \mathrm{~mol} \%)$ or $\mathbf{P 3}(2.1 \mathrm{mg}, 2.0 \mu \mathrm{mol}, 1.0 \mathrm{~mol} \%)$ or P2 (1.8 mg, $2.0 \mu \mathrm{mol}$, $1.0 \mathrm{~mol} \%)$ ]. The reaction tube was transferred into a nitrogen-filled drybox. Base [cesium carbonate (78.2 mg, $240 \mu \mathrm{mol}, 1.20$ equiv) or sodium phenoxide ( $27.9 \mathrm{mg}, 240 \mu \mathrm{mol}, 1.20$ equiv) or potassium phosphate $(50.9 \mathrm{mg}, 240 \mu \mathrm{mol}, 1.20$ equiv)] was added and the reaction tube was sealed. The sealed reaction tube was removed from the drybox. The reaction test tube was evacuated and backfilled with argon by piercing with a needle attached to a Schlenk line (this process was repeated a total of three times). Electrophile [bromobenzene $(21.1 \mu \mathrm{L}, 200 \mu \mathrm{mol}$, 1.00 equiv) or chlorobenzene $(20.4 \mu \mathrm{L}, 200 \mu \mathrm{mol}, 1.00$ equiv) or phenyl trifluoromethanesulfonate $(32.4 \mu \mathrm{L}, 200 \mu \mathrm{mol}, 1.00$ equiv)] and 1,4-dioxane $(400 \mu \mathrm{L})$ were added sequentially to the reaction tube. The reaction test tube was placed in an oil bath that had been preheated to $70{ }^{\circ} \mathrm{C}$. The reaction mixture was stirred and heated at $70{ }^{\circ} \mathrm{C}$ for $2 \mathrm{~h}$. The reaction mixture was allowed to cool over $20 \mathrm{~min}$ to $\mathrm{rt}$. The cooled product mixture was diluted with $\mathrm{CH}_{2} \mathrm{Cl}_{2}(3.00 \mathrm{~mL})$. The diluted product mixture was filtered through Celite and concentrated to dryness. The crude product was purified by automated flash-column chromatography (eluting with $2 \%$ EtOAc-hexanes initially, grading to $20 \%$ EtOAc-hexanes, linear gradient) to afford $\mathbf{6 a}$ as a white solid. 
D) Design of Experiment (DOE) Analysis

DOE analysis was carried out using JMP ${ }^{\circledR}$ Software.

Table 2. Summary of reaction optimization by DOE. ${ }^{a}$ A. Initial analysis of eleven reaction variables.

A.

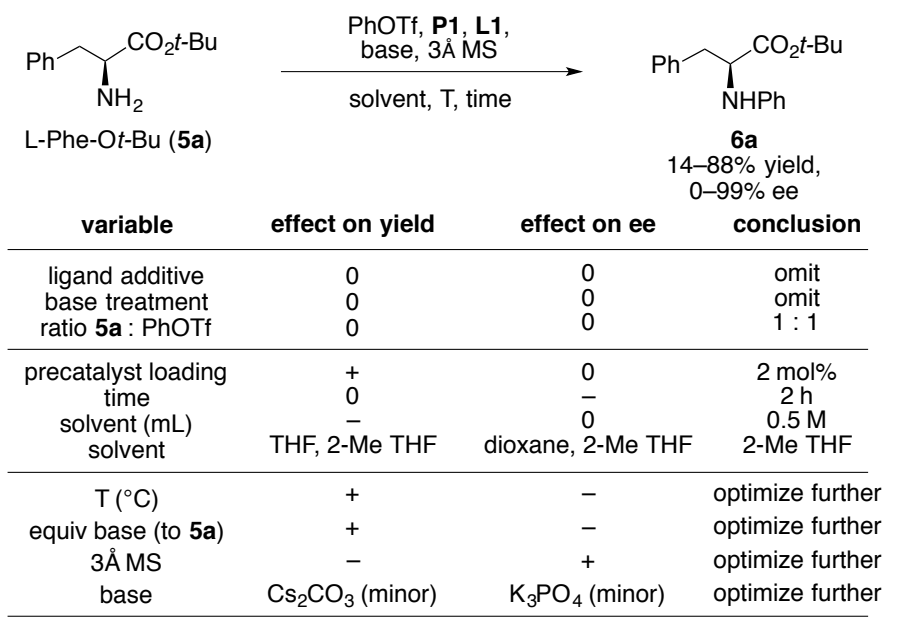

${ }^{a}$ For categorical variables, highest yield/ee obtained with listed entry. For continuous variables: $0=$ variable has no effect on yield/ee, $+=$ highest yield/ee obtained at highest value of variable, $-=$ highest yield/ee obtained at lowest value of variable.

Variable Legend:

ligand additive: 0 (none added), 1 (added same mol\% as the mol\% precatalyst in reaction) precatalyst loading (mol\%): 1 or 5

solvent volume $(\mathrm{mL}): 1$ or 5

$\mathrm{T}\left({ }^{\circ} \mathrm{C}\right)=50$ or 90

time $(\mathrm{h})=2$ or 12

$3 \AA$ MS: 0 (none added) or 1 (50 mg added)

base: $\mathrm{K}_{3} \mathrm{PO}_{4}$ or $\mathrm{Cs}_{2} \mathrm{CO}_{3}$

base treatment: no treatment or finely ground

ratio 5a to phenyl trifluoromethanesulfonate: 0.83 or 1.2

equiv base (to 5a): 1 or 3

solvent: 1,4-dioxane, 2-methyltetrahydrofuran, or THF 
Table S2: Reactions run in initial DOE analysis.

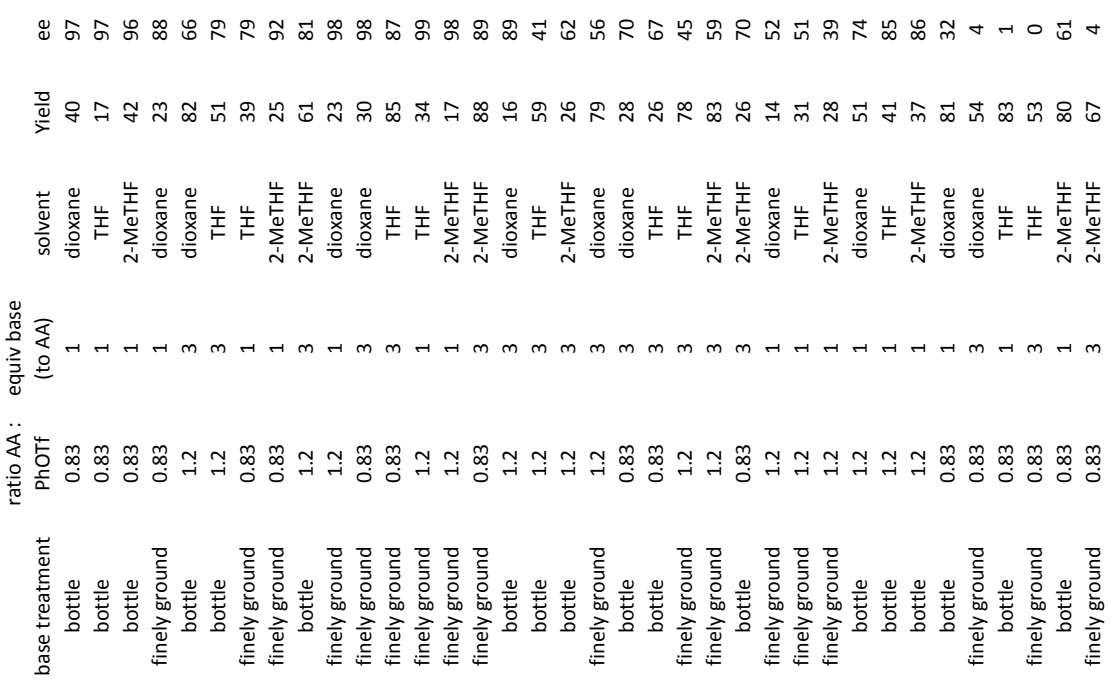

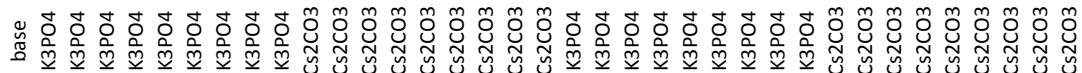

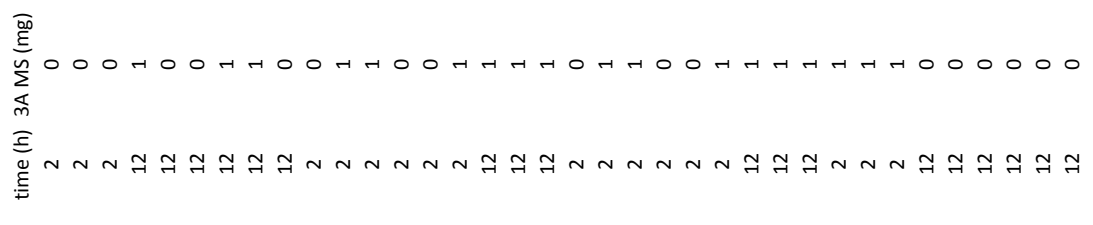

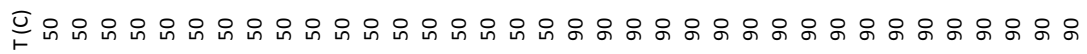

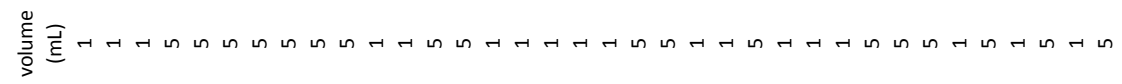

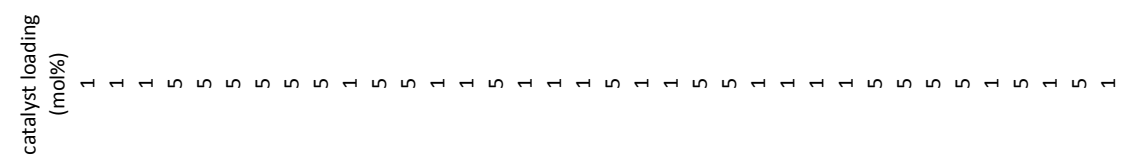

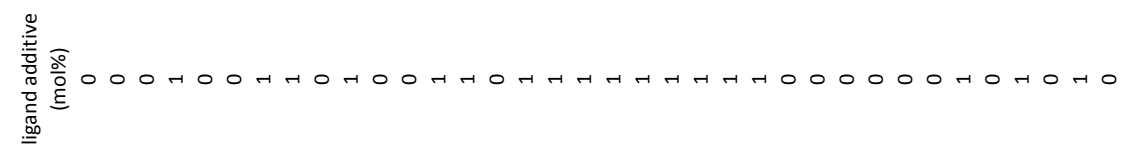

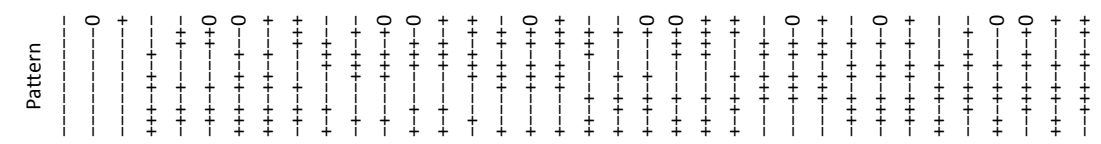


Figure S2: Statistical analysis of 11 reaction variables.

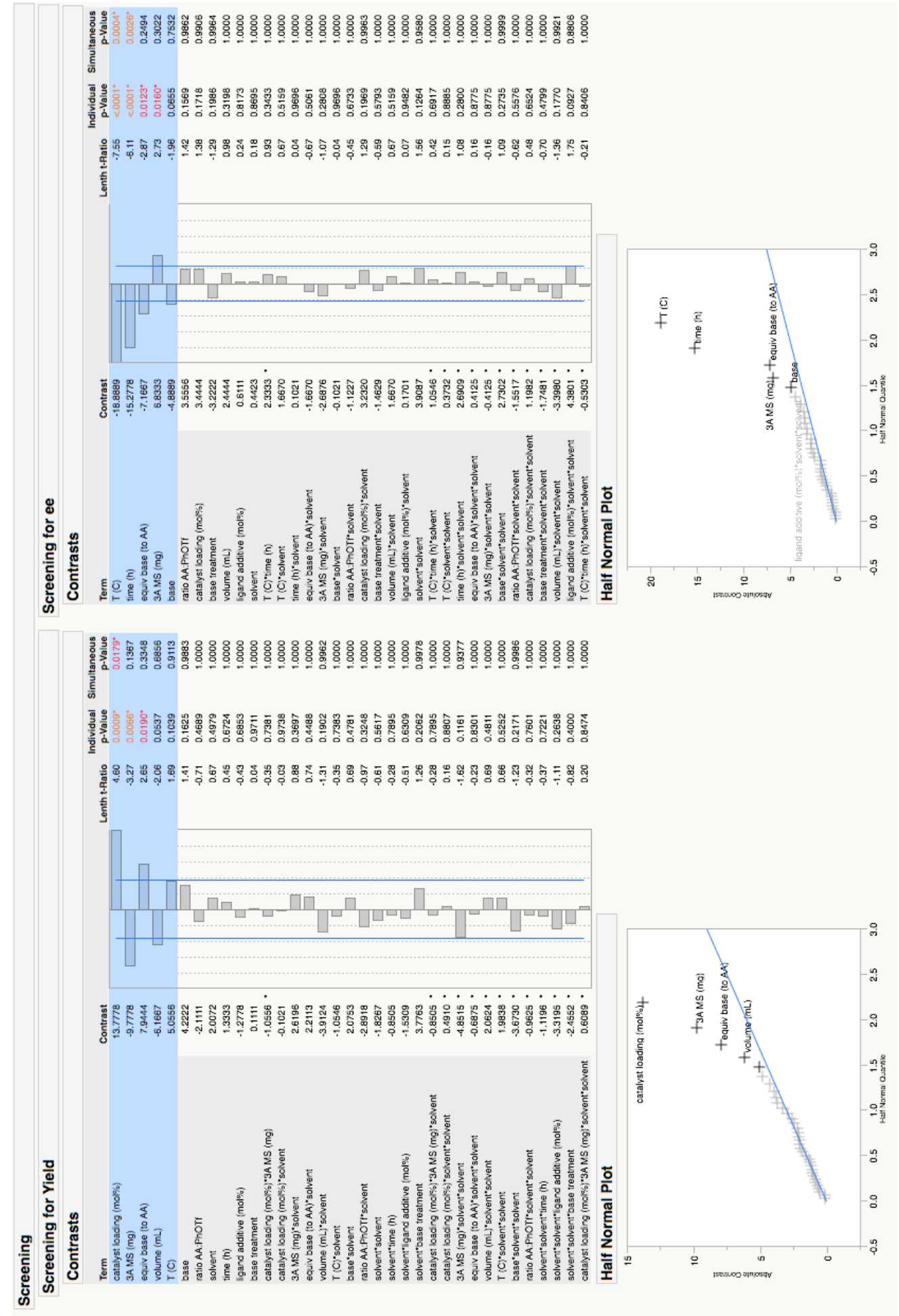


Table 2. Summary of reaction optimization by DOE. ${ }^{a}$ B. Subsequent analysis of four reaction variables.

B.

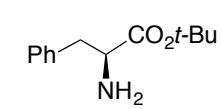

L-Phe-Ot-Bu (5a)

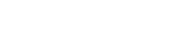

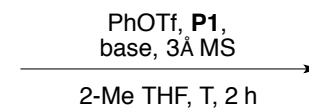

2-Me THF, T, 2 h

$\begin{array}{cc} & 6 a \\ \text { effect on ee } & \begin{array}{c}18-88 \% \text { yield, } \\ 61-99 \% \text { ee } \\ \text { conclusion }\end{array} \\ - & \text { optimize further } \\ - & \text { optimize further } \\ + \text { (minor) } & \text { omit } \\ 0 & \mathrm{Cs}_{2} \mathrm{CO}_{3}\end{array}$

${ }^{a}$ For categorical variables, highest yield/ee obtained with listed entry. For continuous variables: $0=$ variable has no effect on yield/ee, $+=$ highest yield/ee obtained at highest value of variable, $-=$ highest yield/ee obtained at lowest value of variable.

Variable Legend:

$\mathrm{T}\left({ }^{\circ} \mathrm{C}\right)=50,60$, or 70

equiv base (to $\mathbf{5 a}$ ): 1 or 3

$3 \AA \mathrm{MS}(\mathrm{mg})$ : 0 or 50

base: $\mathrm{K}_{3} \mathrm{PO}_{4}$ or $\mathrm{Cs}_{2} \mathrm{CO}_{3}$

Table S3. Subset of reactions from subsequent DOE analysis.

\begin{tabular}{|c|c|c|c|c|c|c|c|c|}
\hline \multirow[b]{2}{*}{ entry } & \multirow[b]{2}{*}{$\mathbf{T}$} & \multirow[b]{2}{*}{ base } & \multirow[b]{2}{*}{$\begin{array}{c}\text { equiv base } \\
\text { (to 5a) }\end{array}$} & \multirow[b]{2}{*}{$3 \AA$ MS } & \multicolumn{2}{|c|}{$2 \mathrm{~mol} \%$ precatalyst } & \multicolumn{2}{|c|}{5 mol\% precatalyst } \\
\hline & & & & & yield & ee & yield & ee \\
\hline 1 & $50^{\circ} \mathrm{C}$ & $\mathrm{Cs}_{2} \mathrm{CO}_{3}$ & 1 & $0 \mathrm{mg}$ & $64 \%$ & $91 \%$ & $89 \%$ & $94 \%$ \\
\hline 2 & $50^{\circ} \mathrm{C}$ & $\mathrm{Cs}_{2} \mathrm{CO}_{3}$ & 1 & $50 \mathrm{mg}$ & $51 \%$ & $95 \%$ & $80 \%$ & $92 \%$ \\
\hline 3 & $50^{\circ} \mathrm{C}$ & $\mathrm{Cs}_{2} \mathrm{CO}_{3}$ & 3 & $0 \mathrm{mg}$ & $69 \%$ & $89 \%$ & $93 \%$ & $91 \%$ \\
\hline 4 & $50^{\circ} \mathrm{C}$ & $\mathrm{Cs}_{2} \mathrm{CO}_{3}$ & 3 & $50 \mathrm{mg}$ & $69 \%$ & $92 \%$ & $95 \%$ & $94 \%$ \\
\hline
\end{tabular}

Yellow indicates optimized reaction conditions. 
Table S4: Reactions run in subsequent DOE analysis.

$\begin{array}{ccccccc}\text { Pattern } & \text { T (C) } & \text { eq base (to AA) } & \text { 3A MS } & \text { base } & \text { yield } & \text { ee } \\ -+++ & 50 & 3 & 50 & \mathrm{~K} 3 \mathrm{PO} 4 & 18 & 97 \\ --+- & 50 & 1 & 50 & \mathrm{Cs} 2 \mathrm{CO} 3 & 51 & 95 \\ ---+ & 50 & 1 & 0 & \mathrm{~K} 3 \mathrm{PO} 4 & 27 & 93 \\ -++- & 50 & 3 & 50 & \mathrm{Cs2CO3} & 69 & 92 \\ --++ & 50 & 1 & 50 & \mathrm{~K} 3 \mathrm{PO} 4 & 7 & 99 \\ -+-- & 50 & 3 & 0 & \mathrm{Cs} 2 \mathrm{CO} 3 & 69 & 89 \\ -+-+ & 50 & 3 & 0 & \mathrm{~K} 3 \mathrm{PO} 4 & 55 & 89 \\ ---- & 50 & 1 & 0 & \mathrm{Cs2CO3} & 64 & 91 \\ 000- & 60 & 2 & 25 & \mathrm{Cs2CO3} & 71 & 86 \\ 000+ & 60 & 2 & 25 & \mathrm{~K} 3 \mathrm{PO} 4 & 41 & 90 \\ 000- & 60 & 2 & 25 & \mathrm{Cs} 2 \mathrm{CO} 3 & 84 & 87 \\ ++++ & 70 & 3 & 50 & \mathrm{~K} 3 \mathrm{PO} 4 & 60 & 67 \\ +--+ & 70 & 1 & 0 & \mathrm{~K} 3 \mathrm{PO} 4 & 51 & 81 \\ +++- & 70 & 3 & 50 & \mathrm{Cs2CO3} & 77 & 75 \\ +-+- & 70 & 1 & 50 & \mathrm{Cs} 2 \mathrm{CO} 3 & 51 & 86 \\ ++-- & 70 & 3 & 0 & \mathrm{Cs} 2 \mathrm{CO} 3 & 88 & 74 \\ +--- & 70 & 1 & 0 & \mathrm{Cs} 2 \mathrm{CO} 3 & 72 & 69 \\ ++-+ & 70 & 3 & 0 & \mathrm{~K} 3 \mathrm{PO} 4 & 78 & 61 \\ +-++ & 70 & 1 & 50 & \mathrm{~K} 3 \mathrm{PO} 4 & 30 & 90\end{array}$


Figure S3: Statistical analysis of four reaction variables.

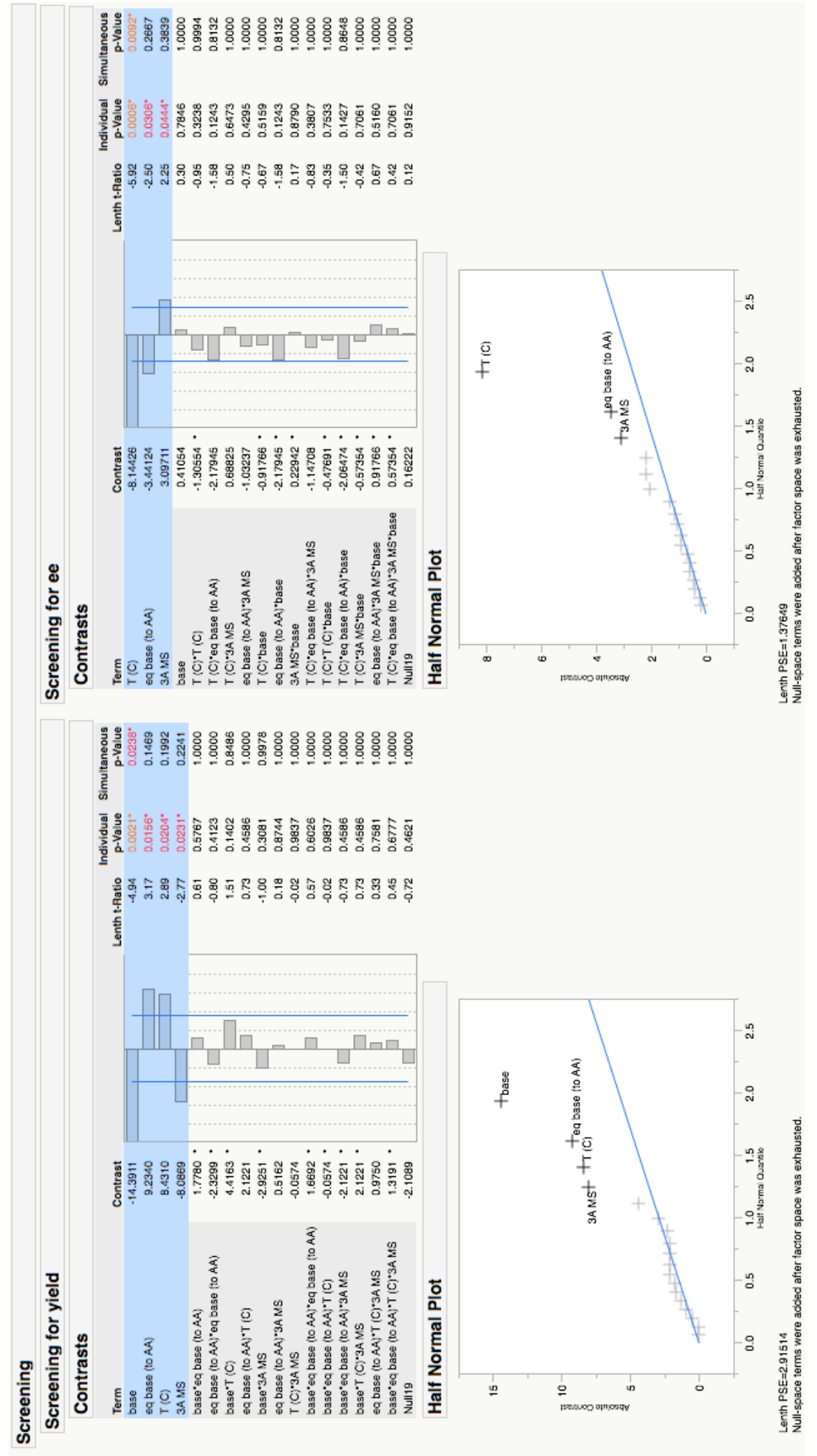


E) Experiments to Determine Mechanism of Racemization

A.

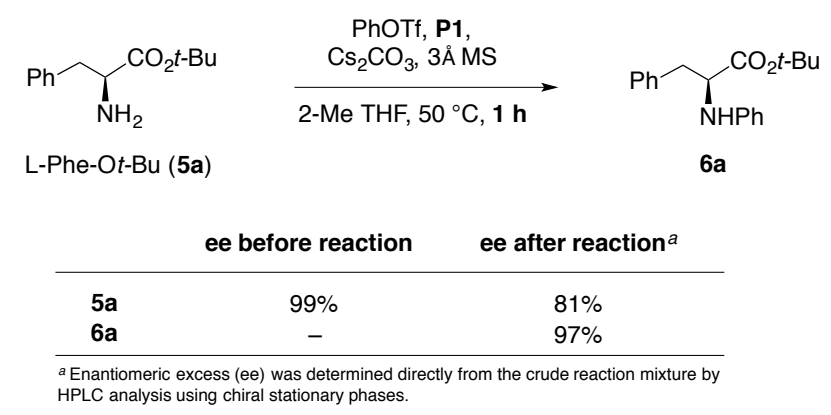

Scheme 3. A. Experiment determining the enantiomeric excess before and after the reaction.

Procedure for Scheme 3A: A $10 \mathrm{~mL}$ screw-cap tube equipped with a stir bar and Teflon septum was charged sequentially with L-Phe-Ot-Bu (5a, $111 \mathrm{mg}, 500 \mu \mathrm{mol}, 1.00$ equiv), P1 (21.4 mg, $30.0 \mu \mathrm{mol}, 5.0 \mathrm{~mol} \%)$, and activated $3 \AA \mathrm{MS}(50.0 \mathrm{mg})$. The reaction tube was transferred into a nitrogen-filled drybox. Cesium carbonate ( $489 \mathrm{mg}, 1.50 \mathrm{mmol}, 3.00$ equiv) was added and the reaction tube was sealed. The sealed reaction tube was removed from the drybox. The reaction test tube was evacuated and backfilled with argon by piercing with a needle attached to a Schlenk line (this process was repeated a total of three times). Phenyl trifluoromethanesulfonate $(81.0 \mu \mathrm{L}$, $500 \mu \mathrm{mol}, 1.00$ equiv) and 2-methyltetrahydrofuran $(1.00 \mathrm{~mL})$ were added sequentially to the reaction tube. The reaction test tube was placed in an oil bath that had been preheated to $50{ }^{\circ} \mathrm{C}$. The reaction mixture was stirred and heated at $50{ }^{\circ} \mathrm{C}$ for $1 \mathrm{~h}$. The reaction mixture was allowed to cool over $20 \mathrm{~min}$ to rt. The cooled product mixture was diluted with $\mathrm{CH}_{2} \mathrm{Cl}_{2}(5.00 \mathrm{~mL})$. The diluted product mixture was filtered through Celite and concentrated to dryness. The crude product mixture was analyzed by HPLC. The crude product mixture was then purified by automated flash-column chromatography (eluting with $8 \%$ ether-hexanes initially, grading to $100 \%$ ether-hexanes, linear gradient, followed by elution with $1 \% \mathrm{MeOH}-$ ether, grading to $10 \%$ $\mathrm{MeOH}-$ ether, linear gradient) to afford $\mathbf{6 a}$ as a white solid. Yield: $116 \mathrm{mg}, 78 \%$. 
B.

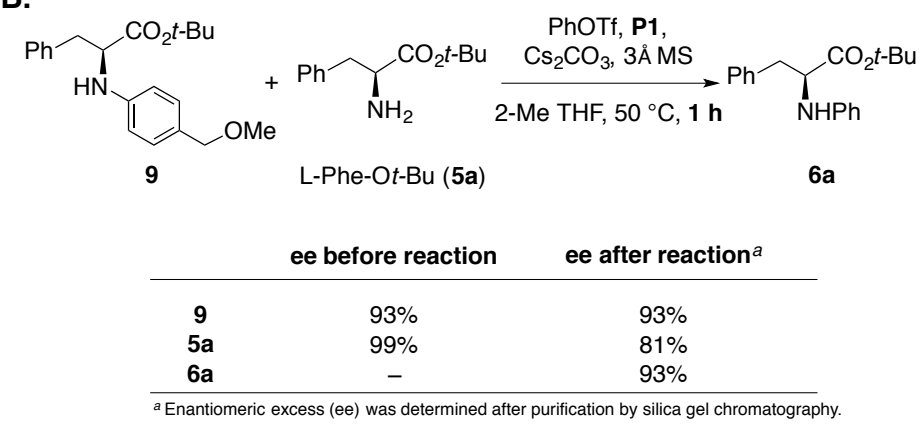

Scheme 3. B. Experiment to test for product racemization with exogenous and different product added.

Procedure for Scheme 3B: A $10 \mathrm{~mL}$ screw-cap tube equipped with a stir bar and Teflon septum was charged sequentially with the $N$-arylation product 9, (85.4 mg, $250 \mu \mathrm{mol}, 1.00$ equiv), L-Phe$\mathrm{O} t$-Bu (5a, $55.3 \mathrm{mg}, 250 \mu \mathrm{mol}, 1.00$ equiv), $\mathbf{P 1}(10.7 \mathrm{mg}, 10.0 \mu \mathrm{mol}, 5.0 \mathrm{~mol} \%)$, and activated $3 \AA$ MS $(25.0 \mathrm{mg})$. The reaction tube was transferred into a nitrogen-filled drybox. Cesium carbonate $(244 \mathrm{mg}, 750 \mu \mathrm{mol}, 3.00$ equiv) was added and the reaction tube was sealed. The sealed reaction tube was removed from the drybox. The reaction test tube was evacuated and backfilled with argon by piercing with a needle attached to a Schlenk line (this process was repeated a total of three times). Phenyl trifluoromethanesulfonate $(40.5 \mu \mathrm{L}, 250 \mu \mathrm{mol}, 1.00$ equiv) and 2-methyltetrahydrofuran $(1.00 \mathrm{~mL})$ were added sequentially to the reaction tube. The reaction test tube was placed in an oil bath that had been preheated to $50{ }^{\circ} \mathrm{C}$. The reaction mixture was stirred and heated at $50{ }^{\circ} \mathrm{C}$ for $1 \mathrm{~h}$. The reaction mixture was allowed to cool over $20 \mathrm{~min}$ to rt. The cooled product mixture was diluted with $\mathrm{CH}_{2} \mathrm{Cl}_{2}(5.00 \mathrm{~mL})$. The diluted product mixture was filtered through Celite and concentrated to dryness. The residue obtained was purified by automated flash-column chromatography (eluting with $8 \%$ ether-hexanes initially, grading to $100 \%$ ether-hexanes, linear gradient, followed by elution with $1 \% \mathrm{MeOH}$ :ether, grading to $10 \% \mathrm{MeOH}$-ether, linear gradient) to afford 9 as a yellow oil $(85.3 \mathrm{mg}, 99 \%$ recovered), L-Phe-O-t-Bu (5a) as a yellow oil (19.7 $\mathrm{mg}, 36 \%$ recovered), and $6 \mathbf{6}$ as a white solid (43.1 mg, 58\%). 


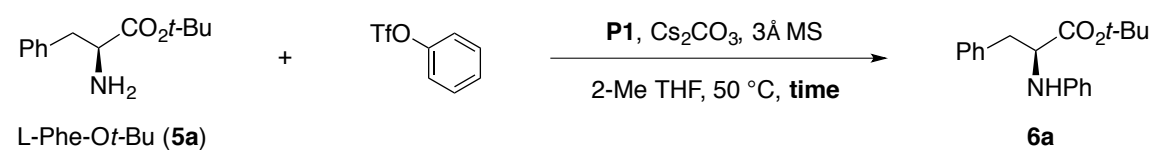

\begin{tabular}{cccc}
\hline \multicolumn{4}{c}{ Table S5. Evaluation of yield and ee over reaction time. } \\
\hline entry & time & yield & ee \\
\hline 1 & $30 \mathrm{~min}$ & $67 \%$ & $98 \%$ \\
2 & $1 \mathrm{~h}$ & $73 \%$ & $97 \%$ \\
3 & $1.5 \mathrm{~h}$ & $90 \%$ & $95 \%$ \\
4 & $2 \mathrm{~h}$ & $92 \%$ & $95 \%$ \\
5 & $4 \mathrm{~h}$ & $96 \%$ & $93 \%$ \\
6 & $16 \mathrm{~h}$ & $99 \%$ & $88 \%$ \\
7 & $10 \mathrm{~d}$ & $91 \%$ & $10 \%$ \\
\hline
\end{tabular}

Procedure for Table S5: A $10 \mathrm{~mL}$ screw-cap tube equipped with a stir bar and Teflon septum was charged sequentially with L-Phe-Ot-Bu (5a, $111 \mathrm{mg}, 500 \mu \mathrm{mol}, 1.00$ equiv), P1 (21.4 mg, $30.0 \mu \mathrm{mol}, 5.0 \mathrm{~mol} \%)$, and activated $3 \AA \mathrm{MS}(50.0 \mathrm{mg})$. The reaction tube was transferred into a nitrogen-filled drybox. Cesium carbonate $(489 \mathrm{mg}, 1.50 \mathrm{mmol}, 3.00$ equiv) was added and the reaction tube was sealed. The sealed reaction tube was removed from the drybox. The reaction test tube was evacuated and backfilled with argon by piercing with a needle attached to a Schlenk line (this process was repeated a total of three times). Phenyl trifluoromethanesulfonate $(81.0 \mu \mathrm{L}$, $500 \mu \mathrm{mol}, 1.00$ equiv) and 2-methyltetrahydrofuran $(1.00 \mathrm{~mL})$ were added sequentially to the reaction tube. The reaction test tube was placed in an oil bath that had been preheated to $50{ }^{\circ} \mathrm{C}$. The reaction mixture was stirred and heated at $50{ }^{\circ} \mathrm{C}$ for the indicated time. The reaction mixture was allowed to cool over 20 min to rt. The cooled product mixture was diluted with $\mathrm{CH}_{2} \mathrm{Cl}_{2}(5.00$ $\mathrm{mL}$ ). The diluted product mixture was filtered through Celite and concentrated to dryness. The crude product was purified by automated flash-column chromatography (eluting with $2 \%$ EtOAchexanes initially, grading to $20 \%$ EtOAc-hexanes, linear gradient) to afford $6 \mathbf{a}$ as a white solid. 
III. Catalog of Spectra

A) Catalog of NMR Spectra

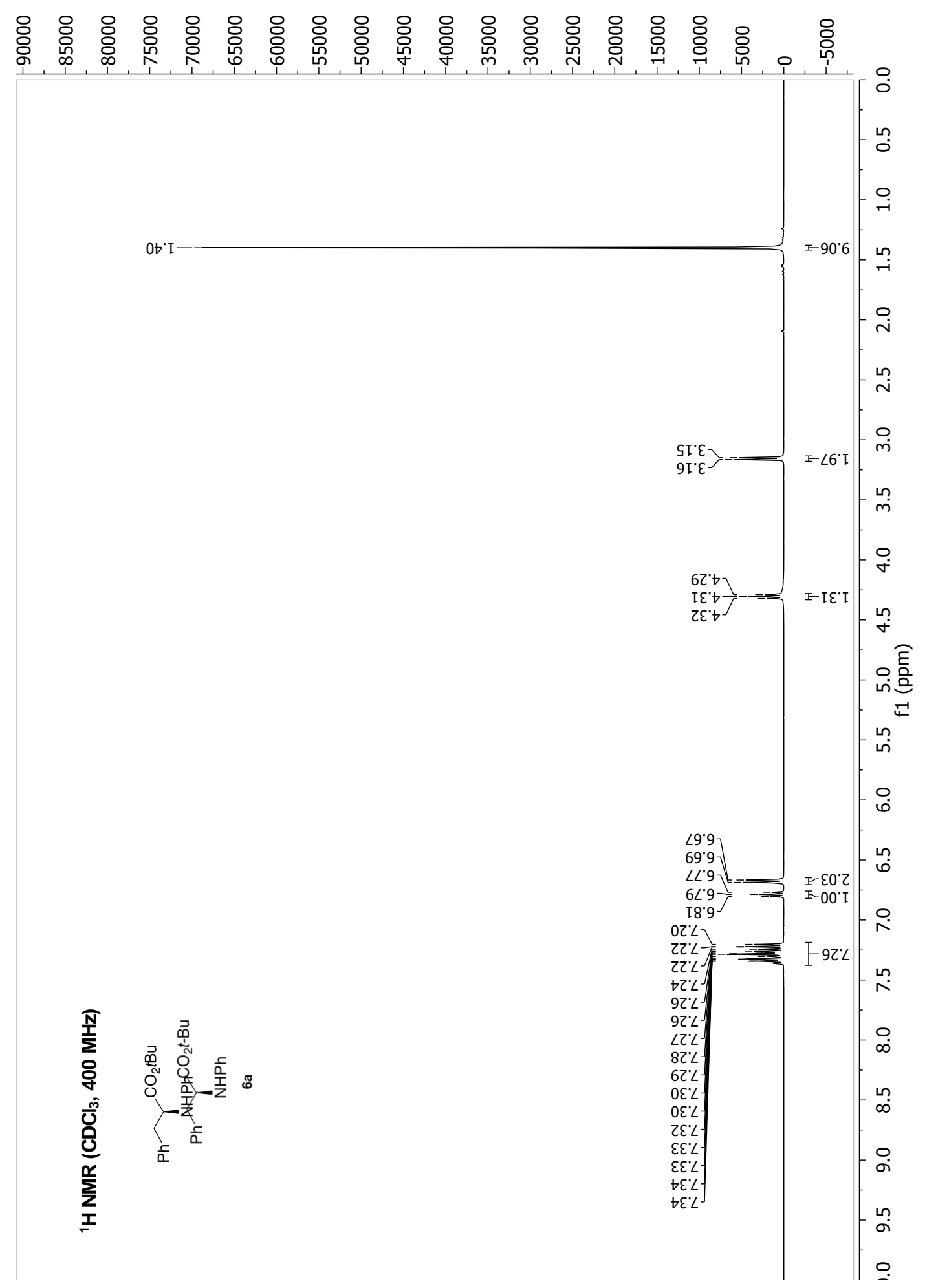




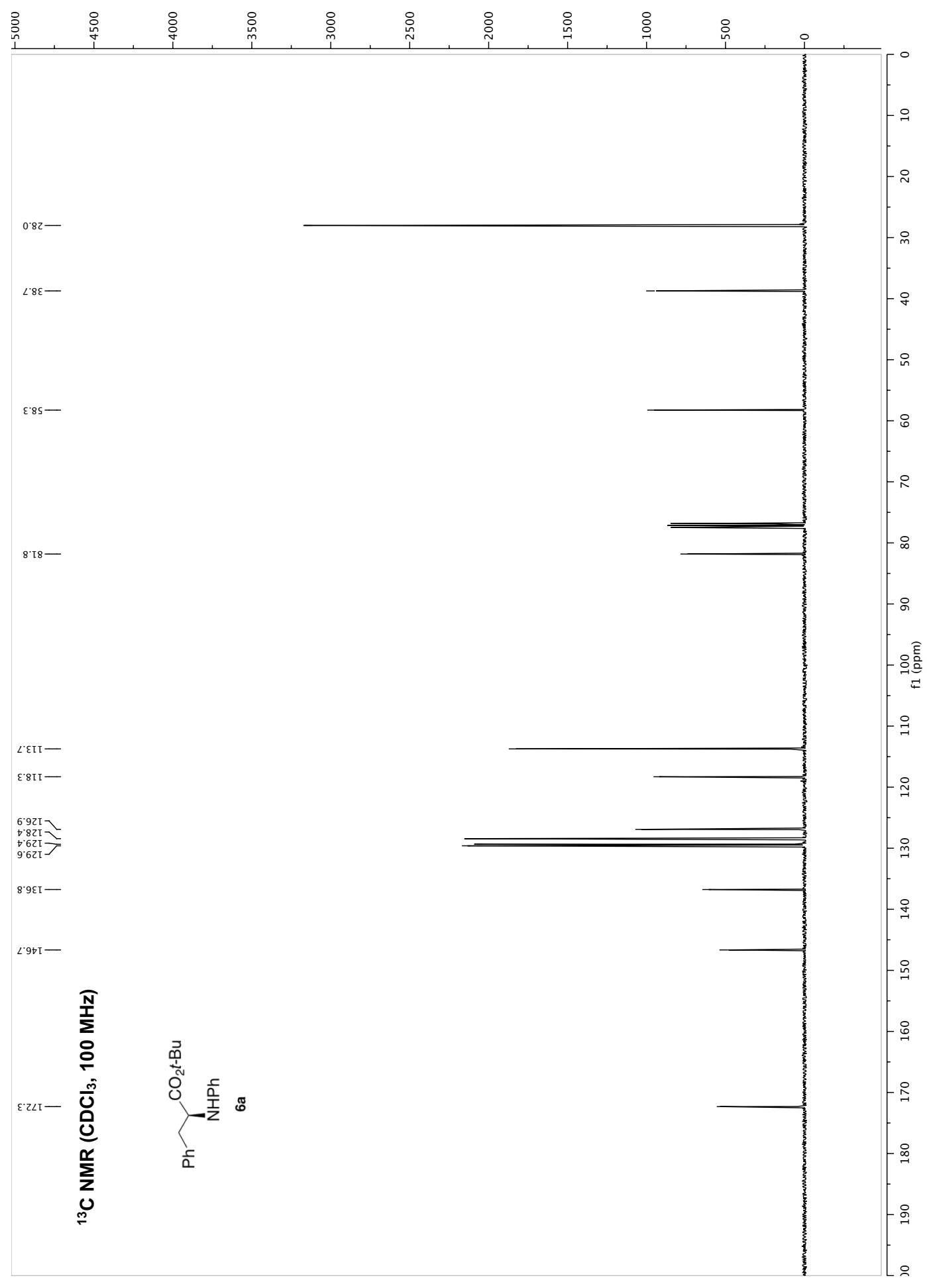




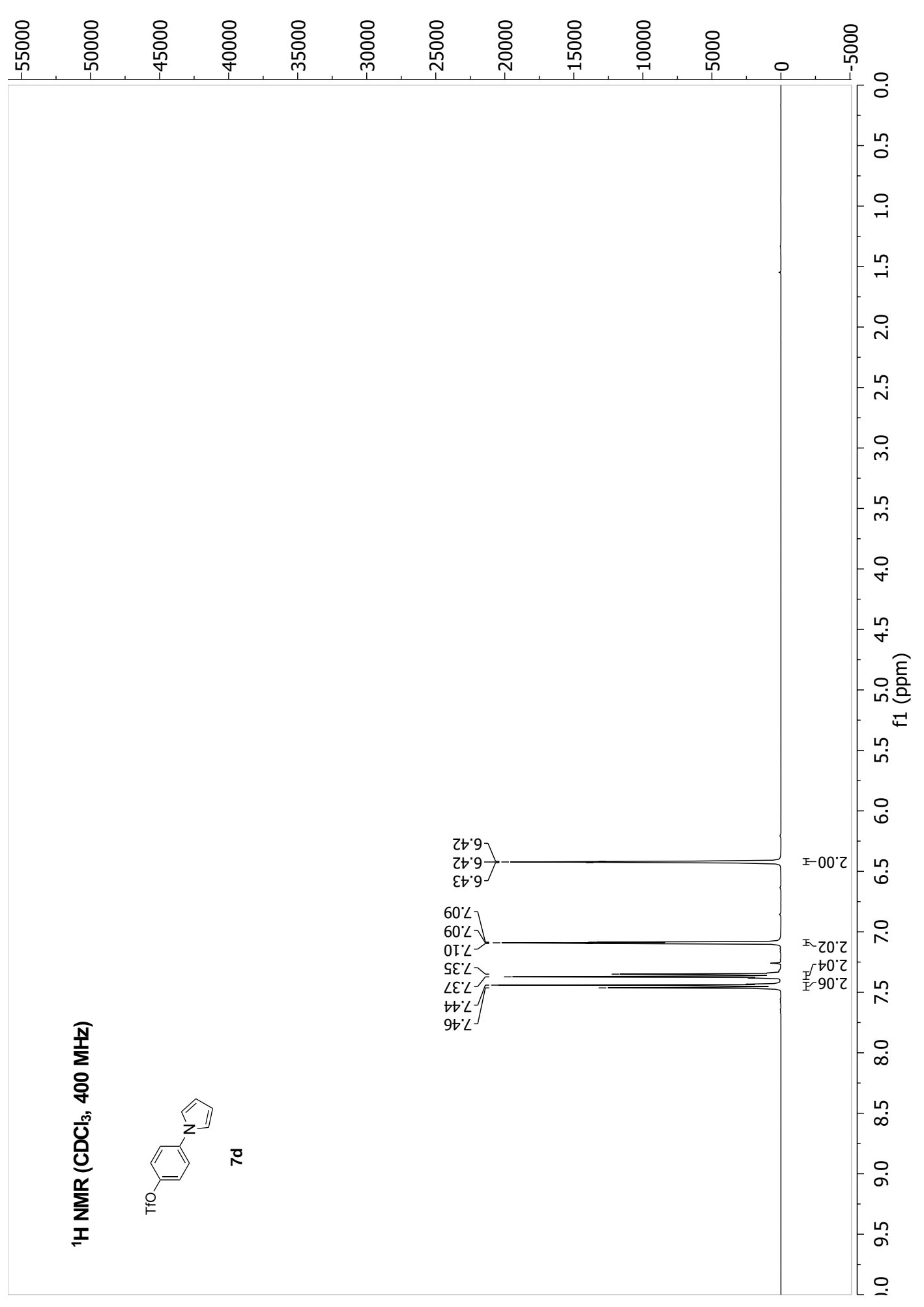




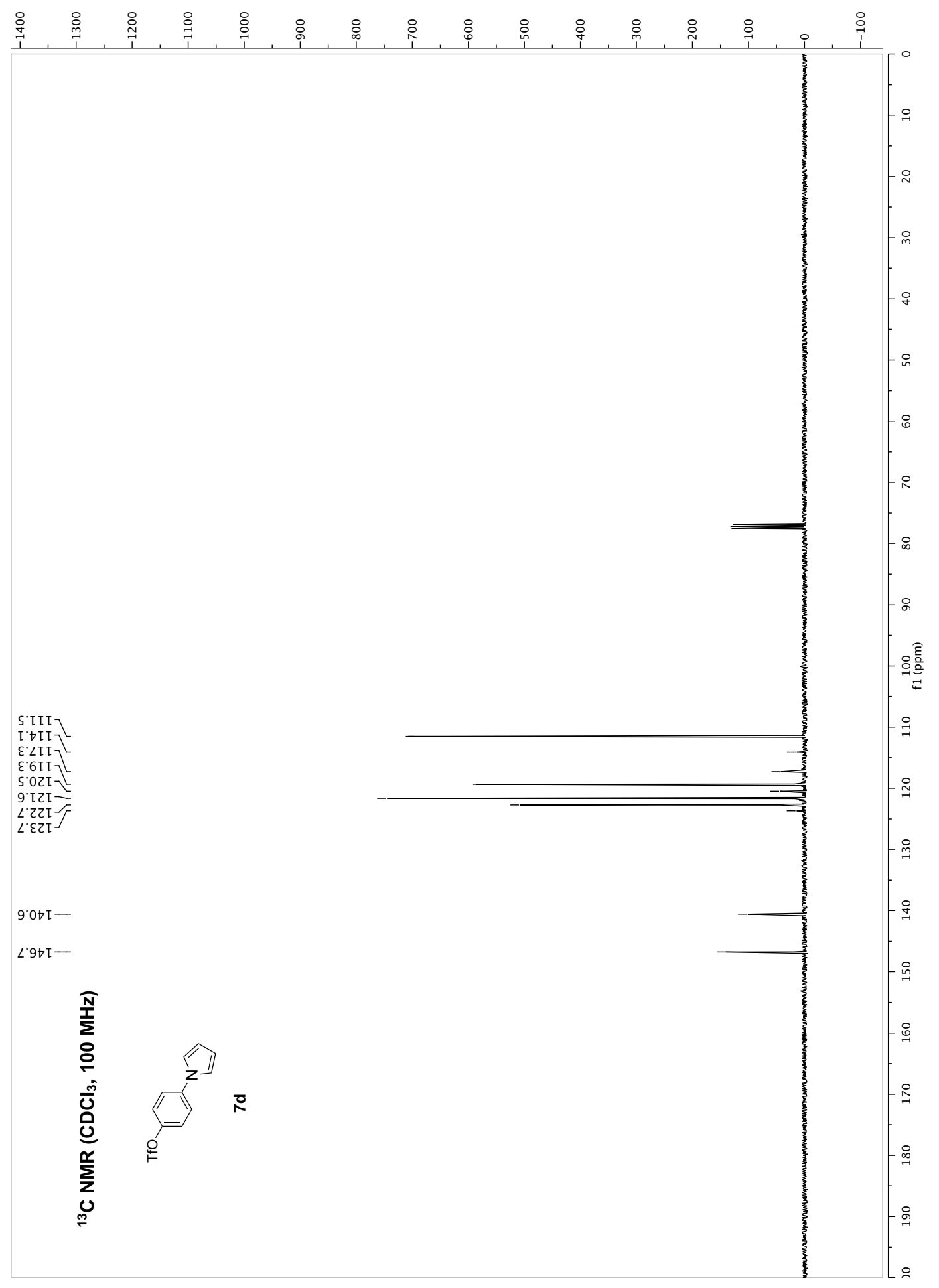




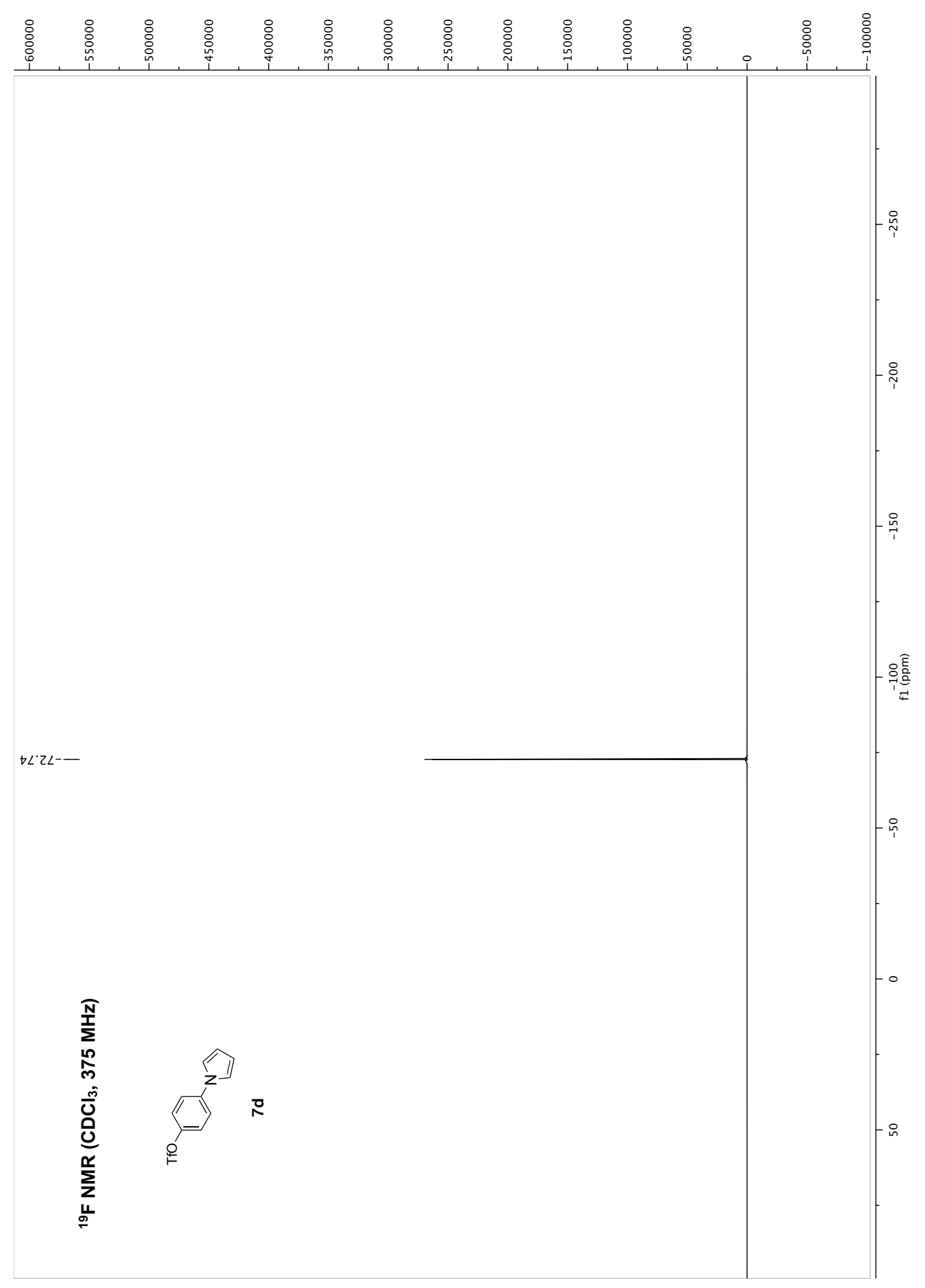




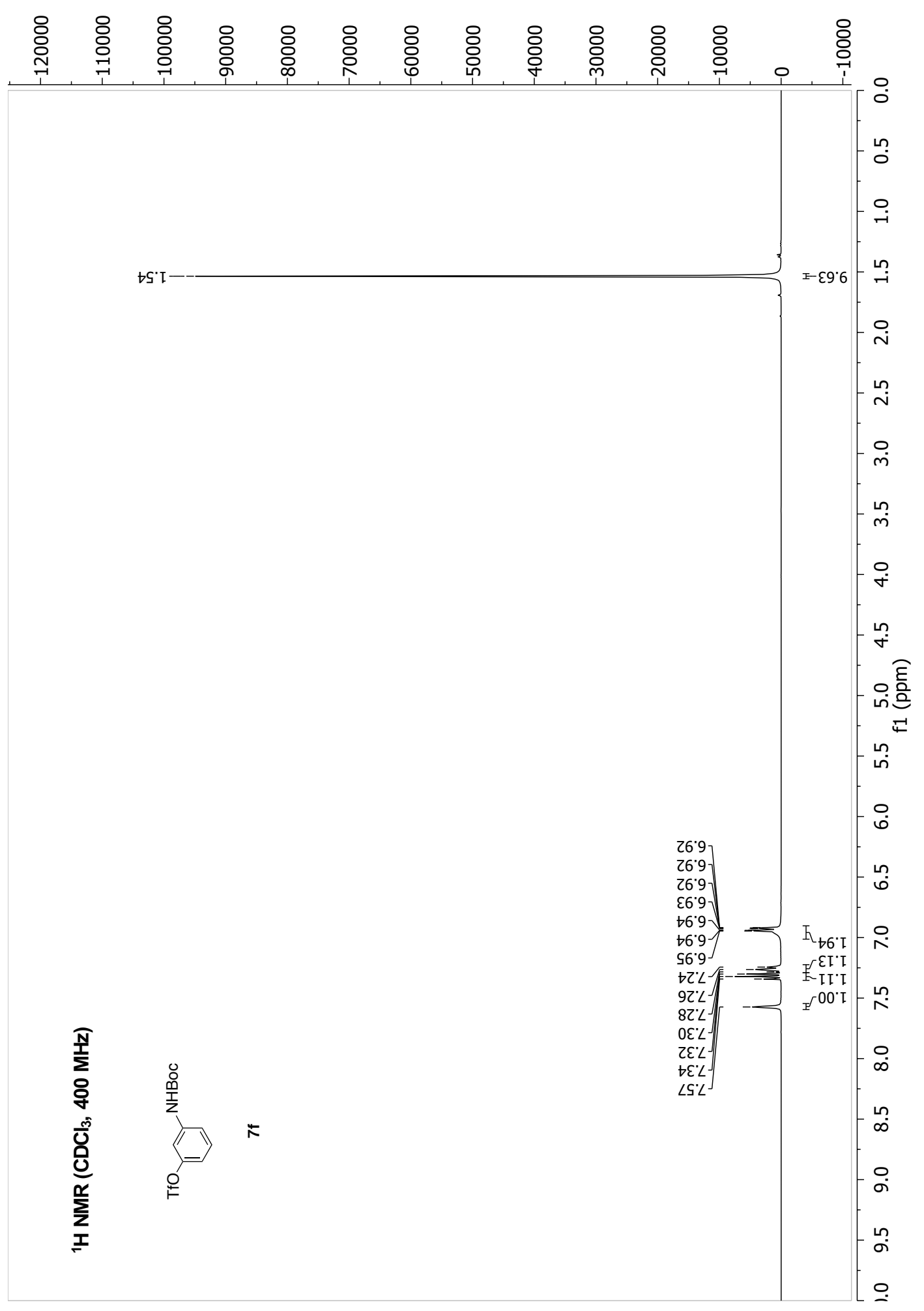




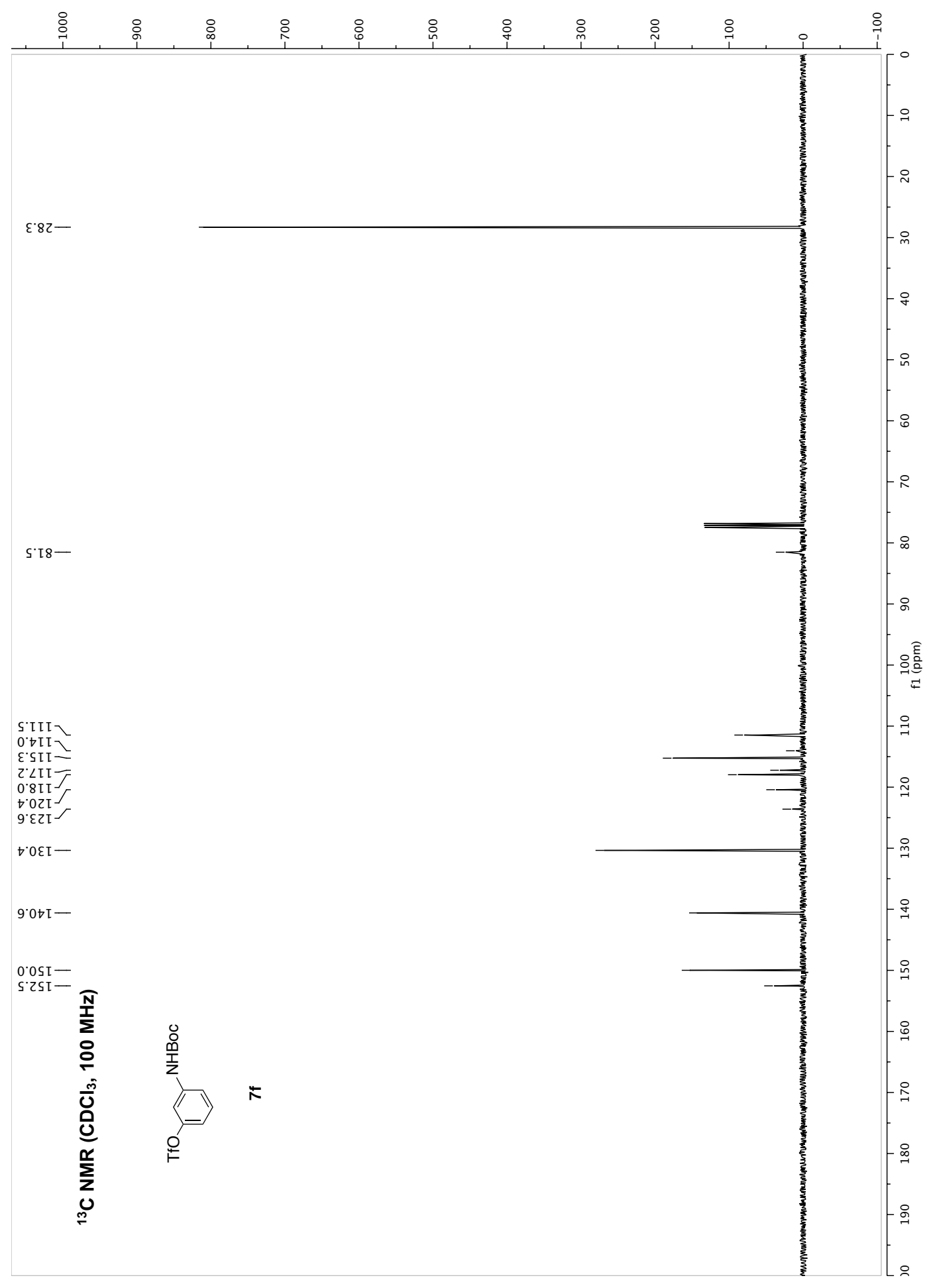




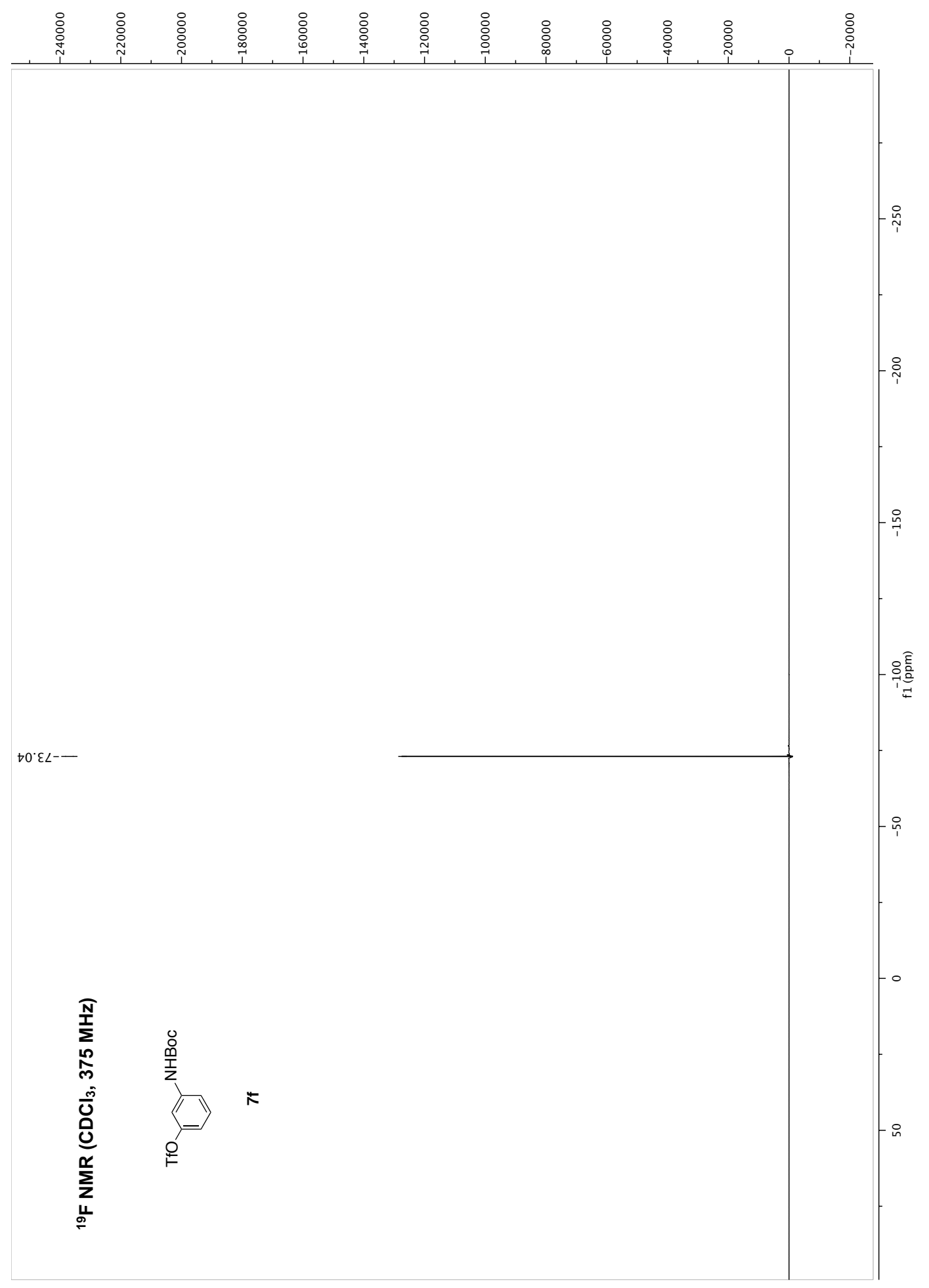




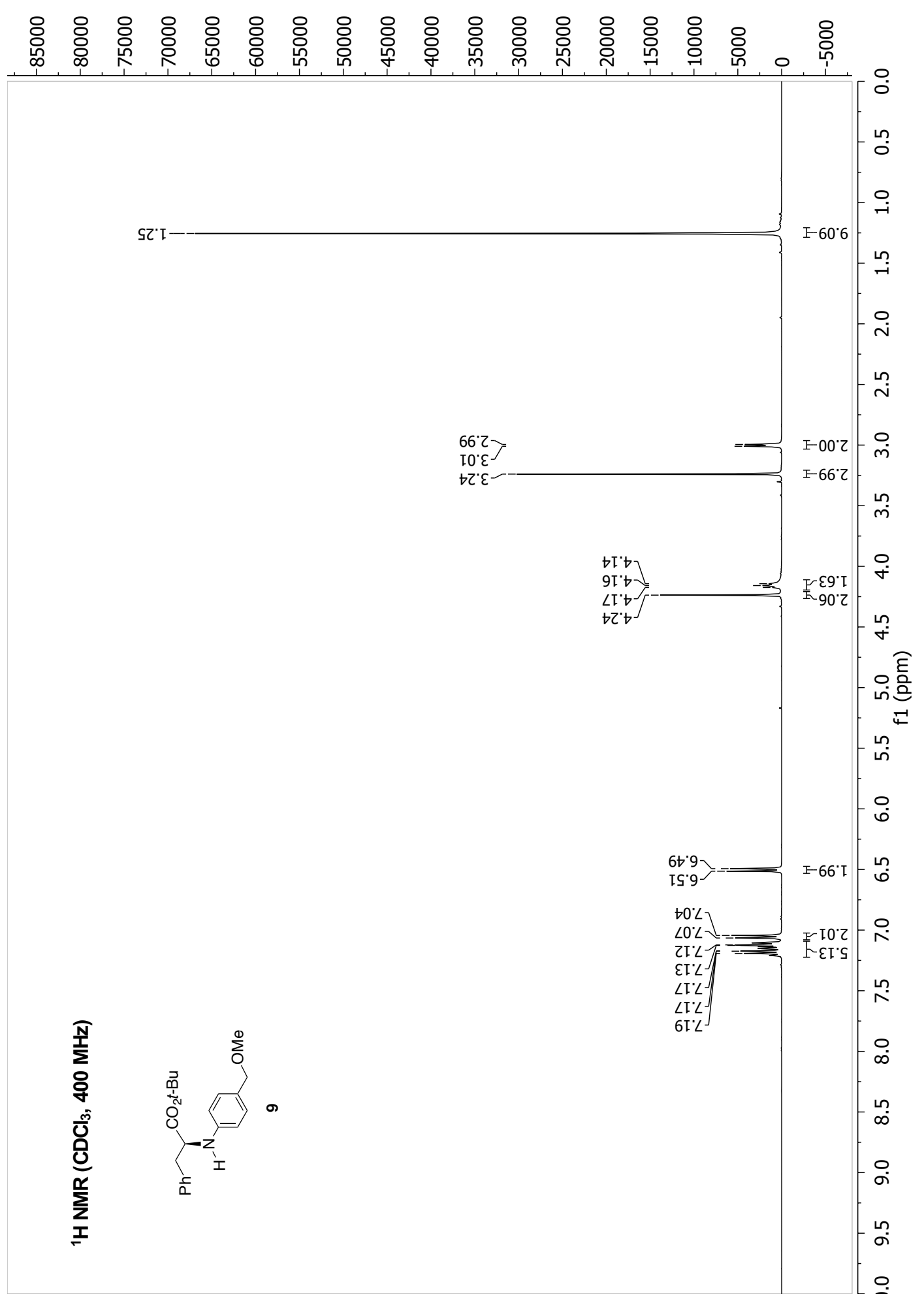




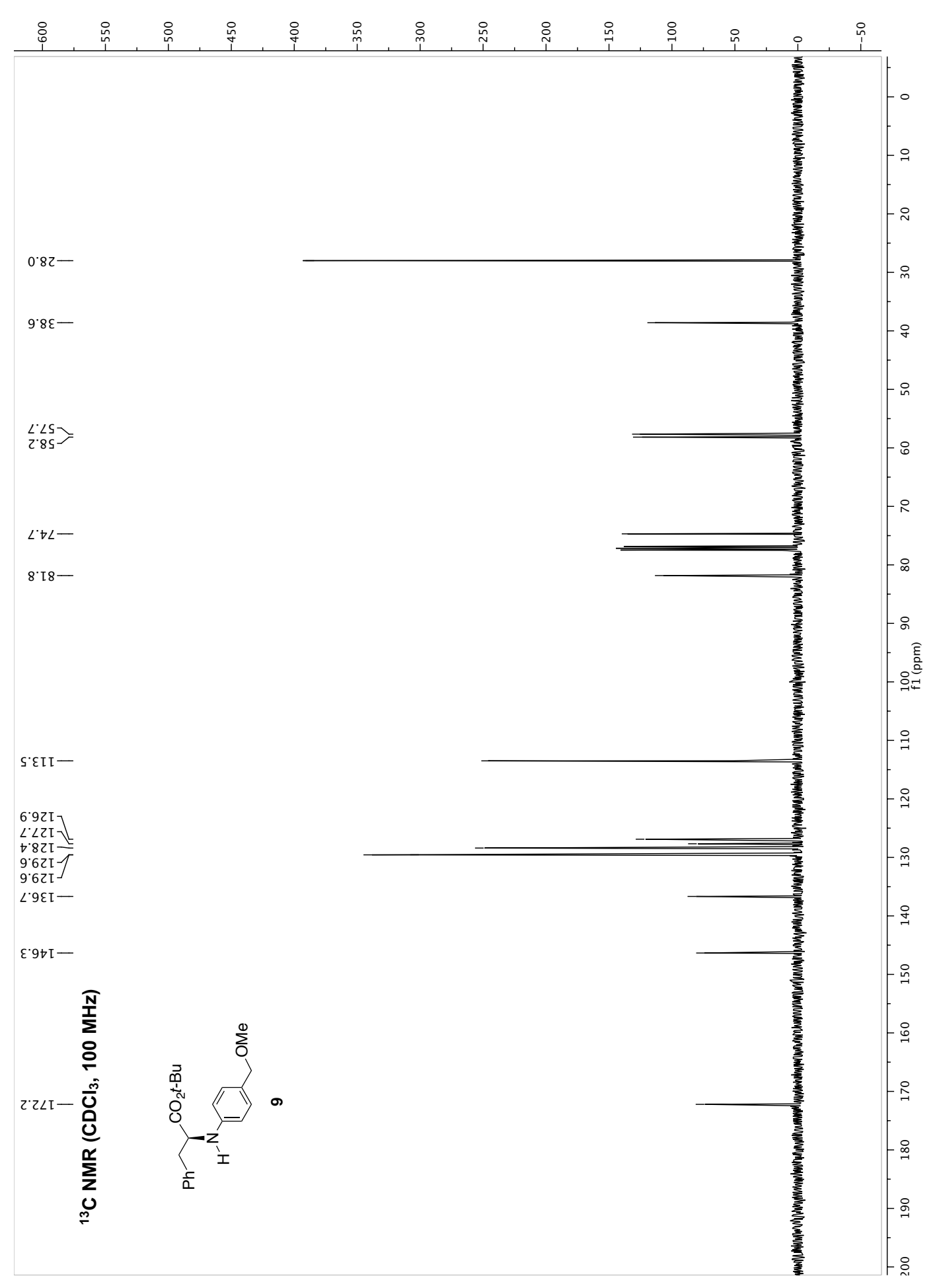




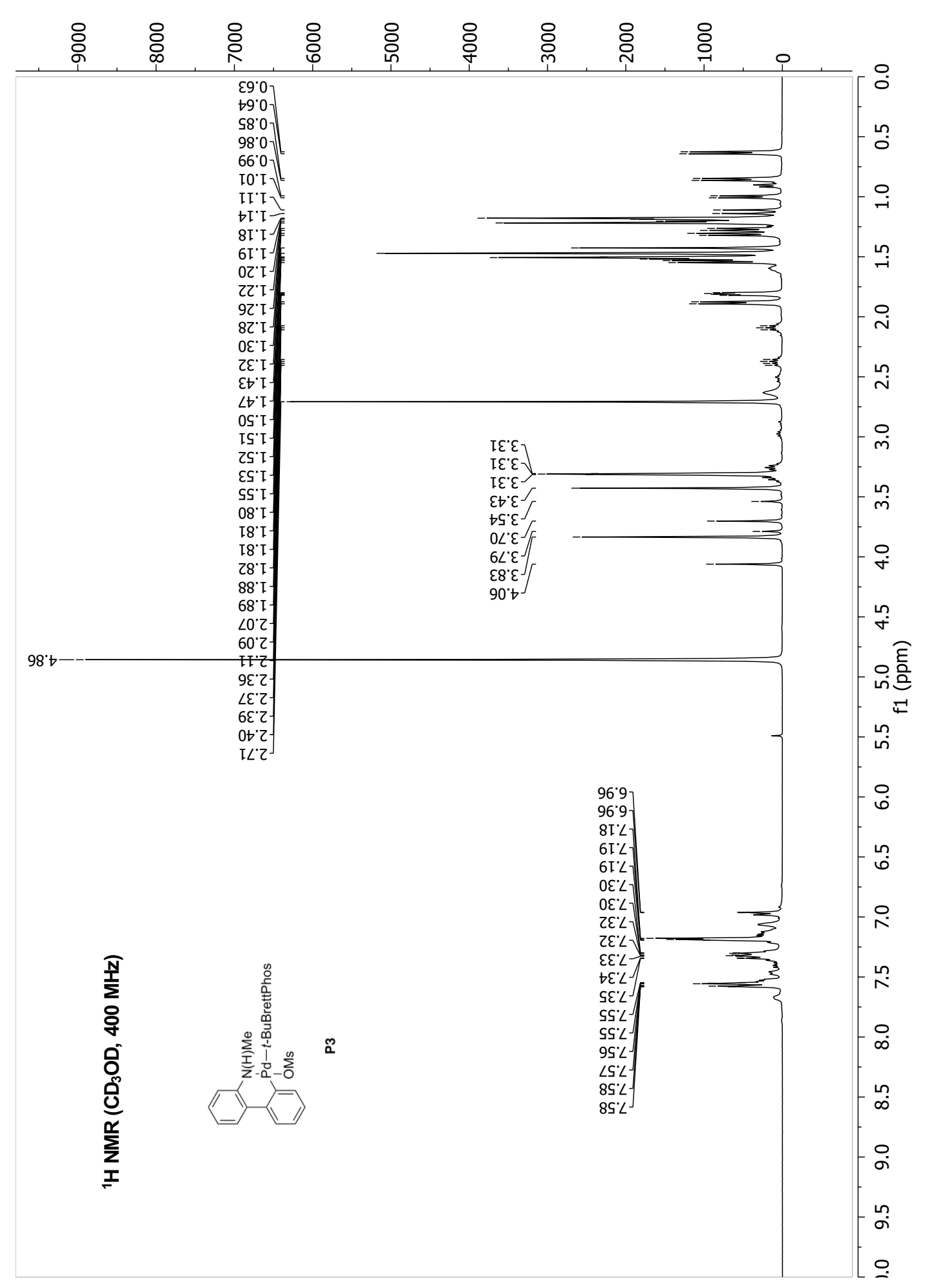




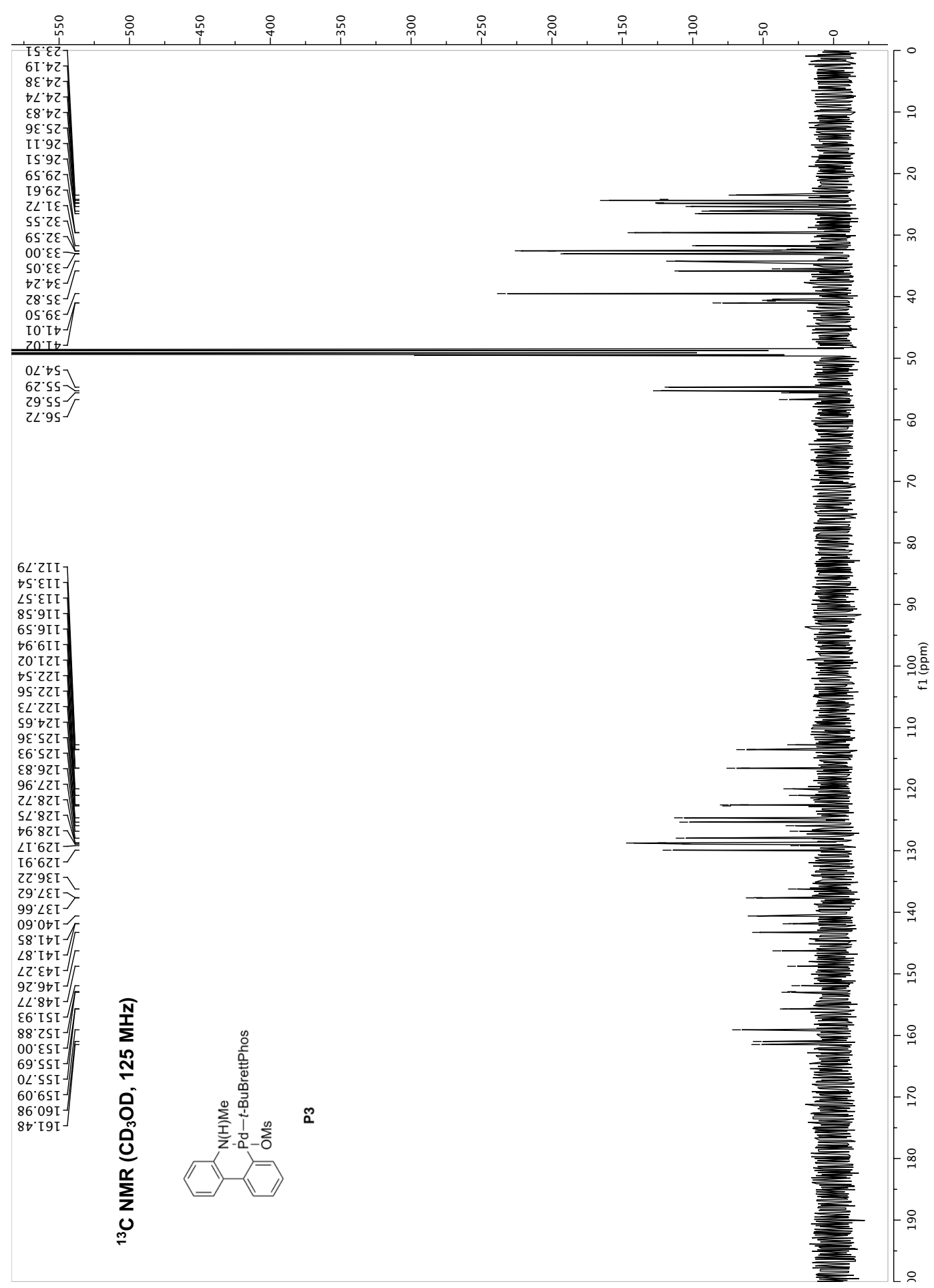




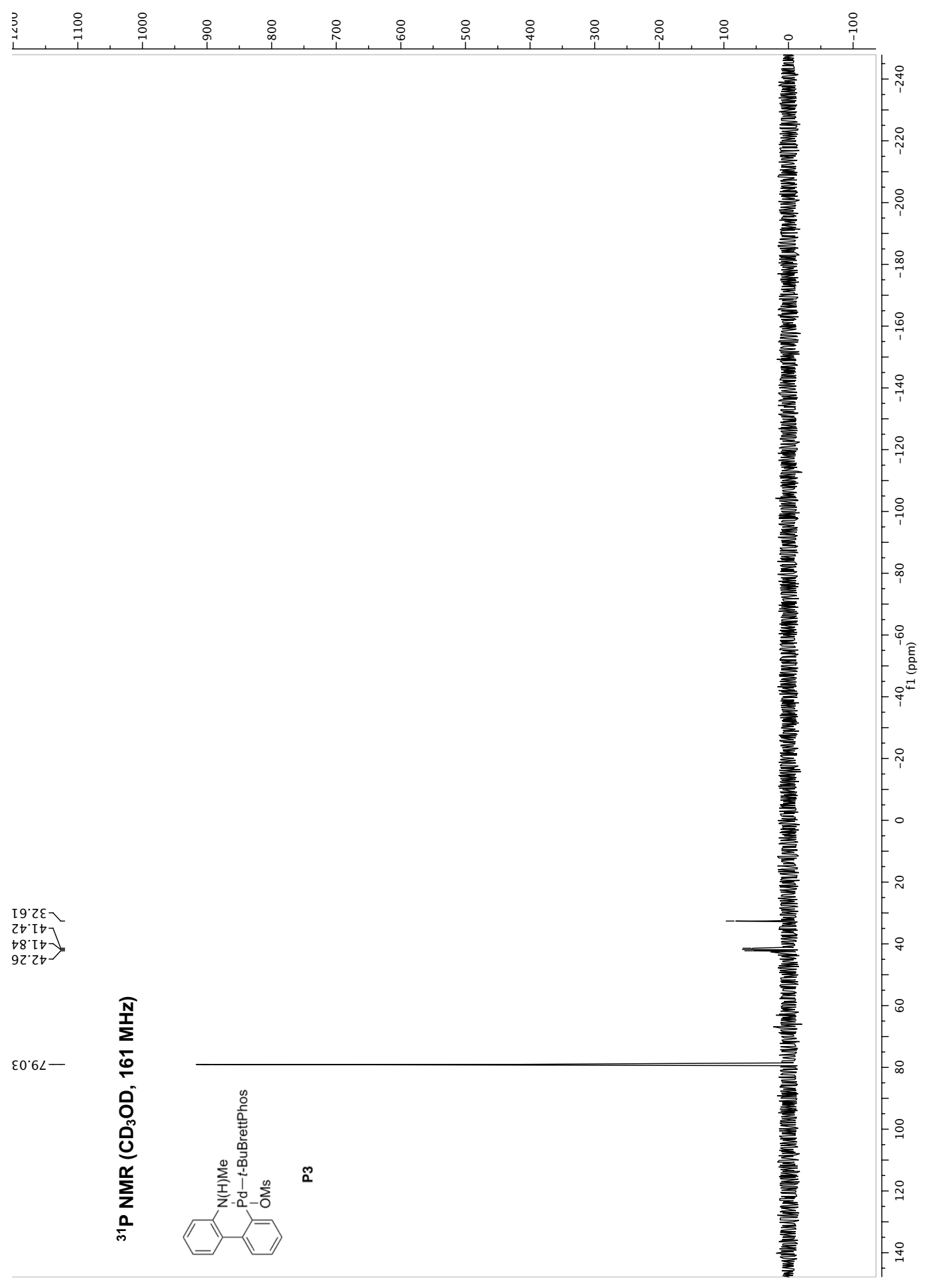




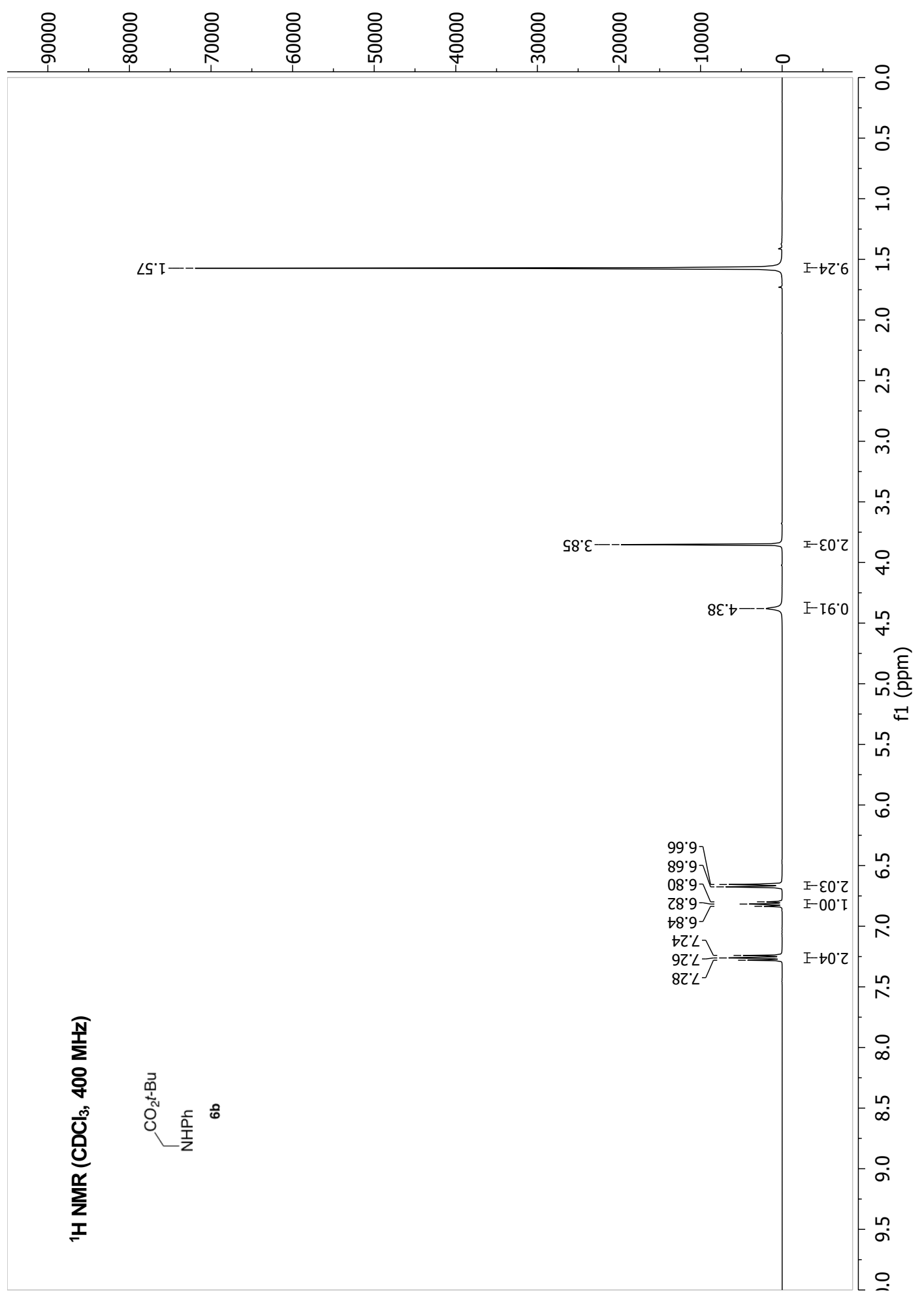




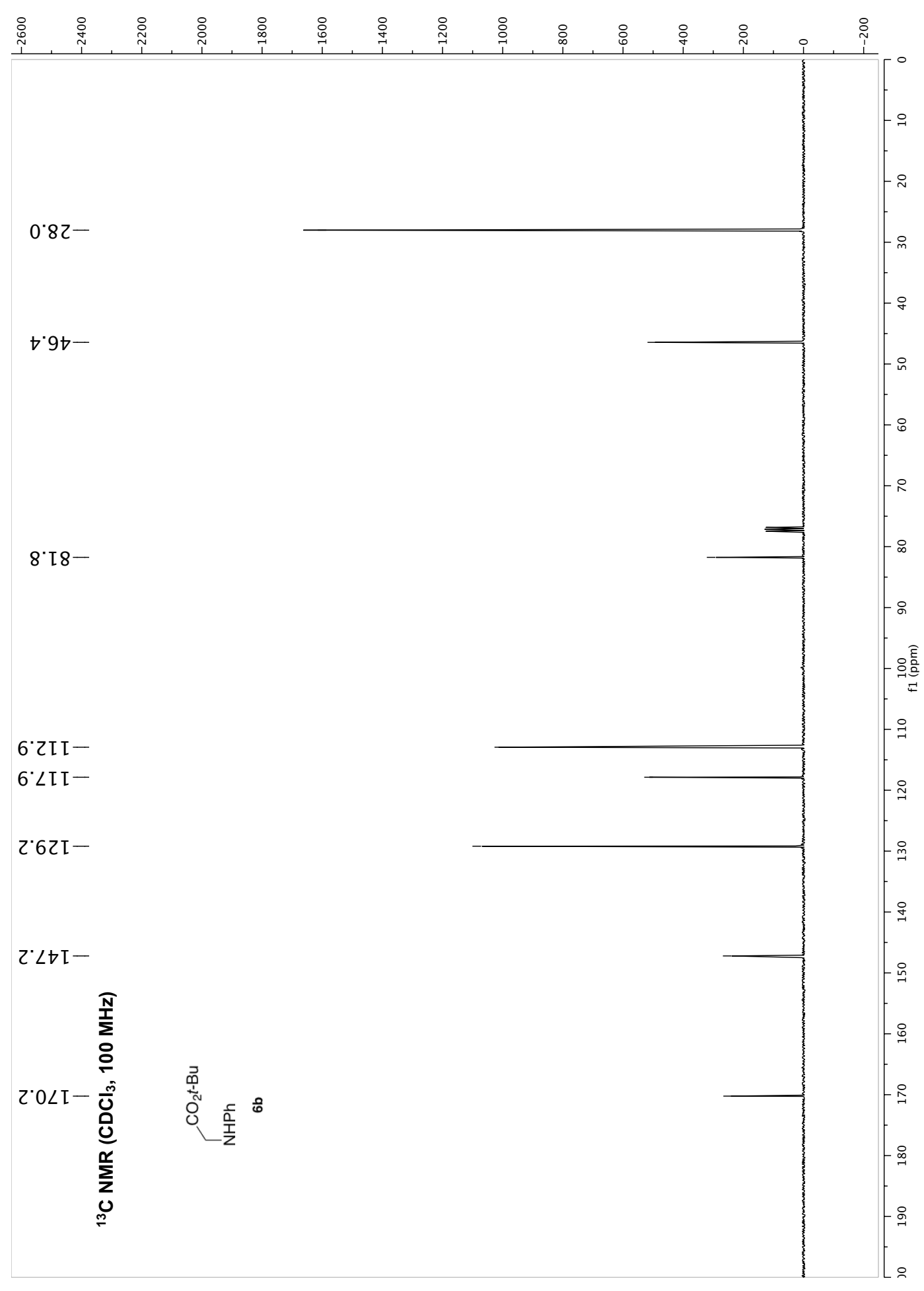




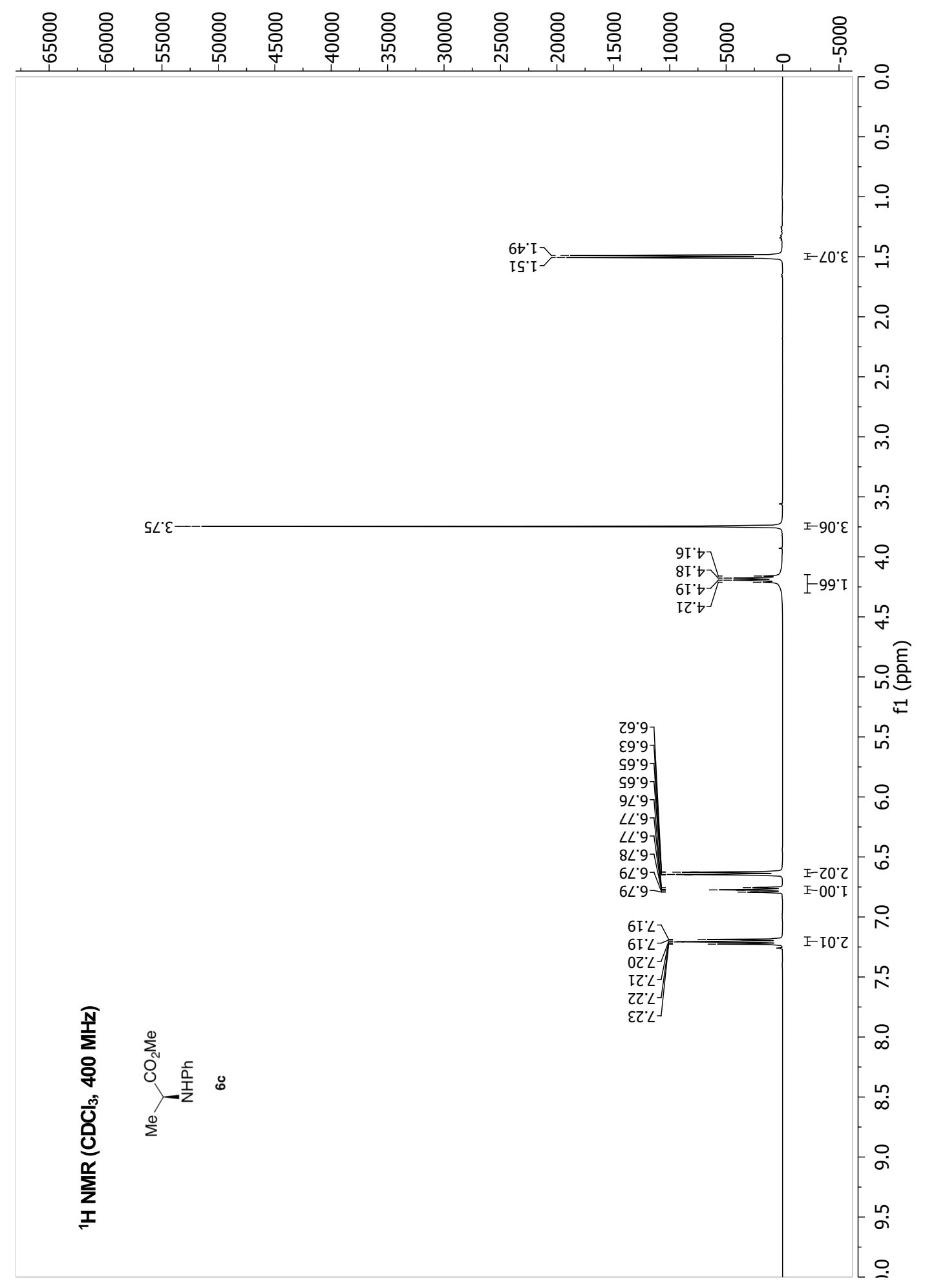




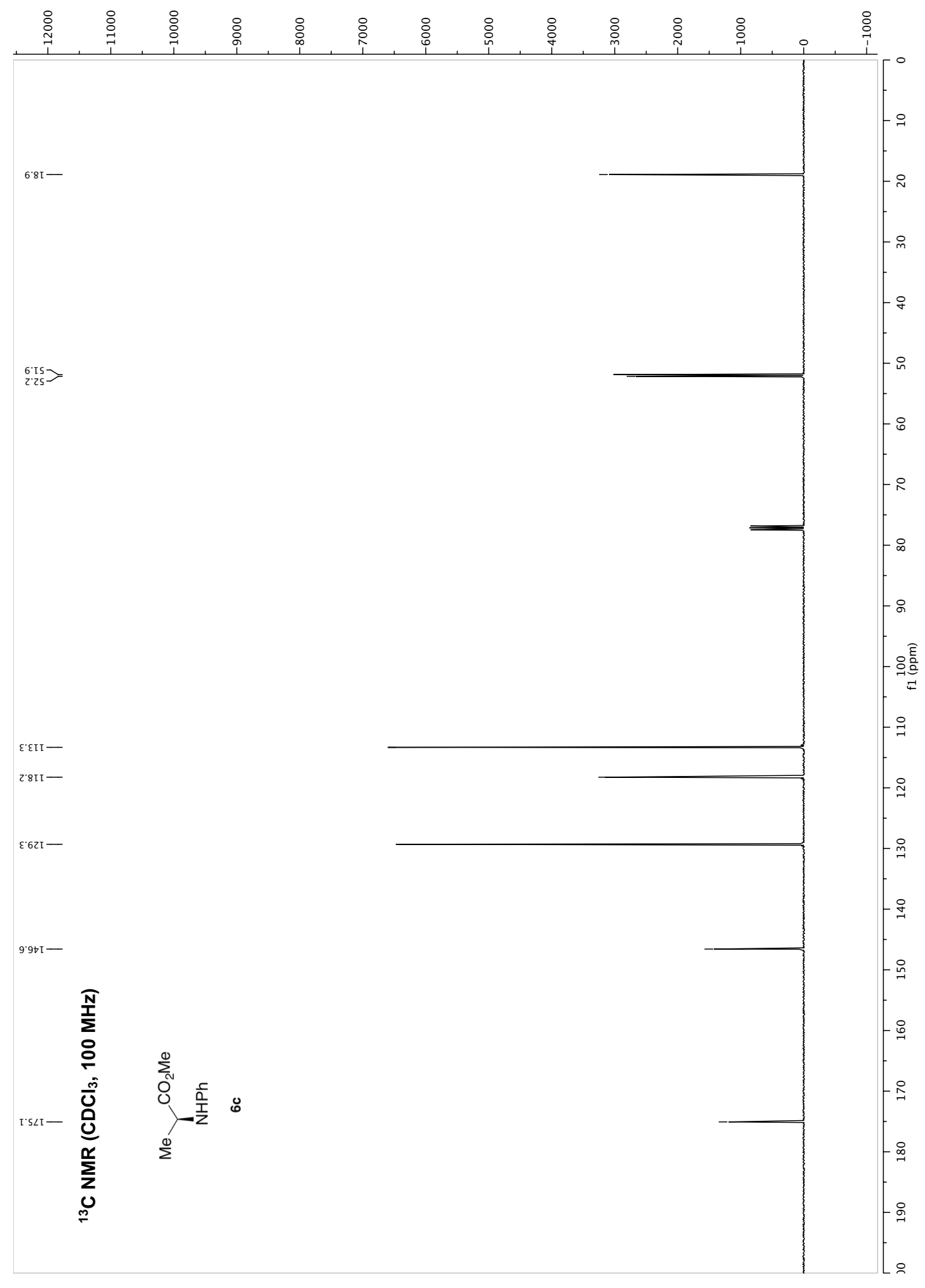




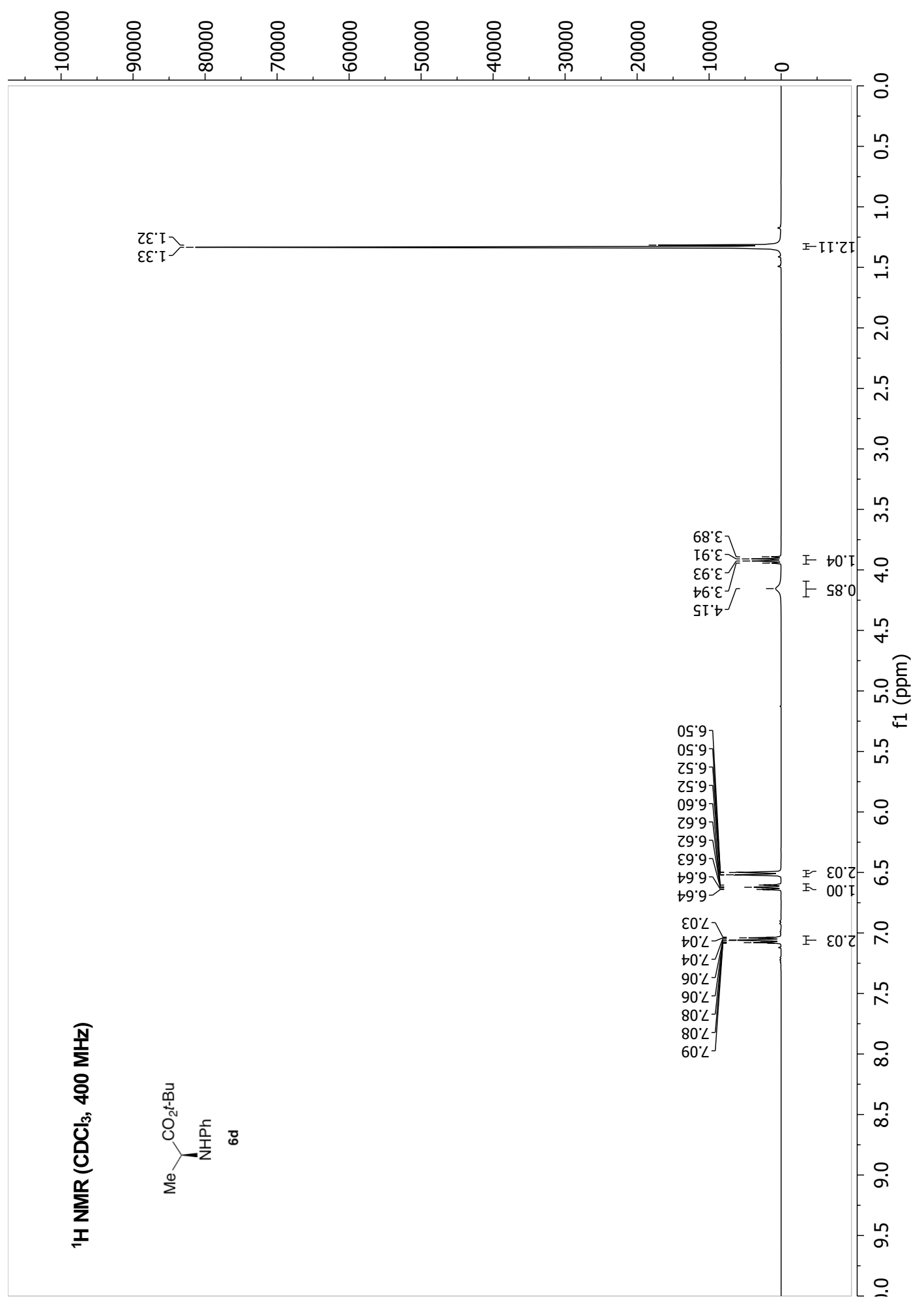




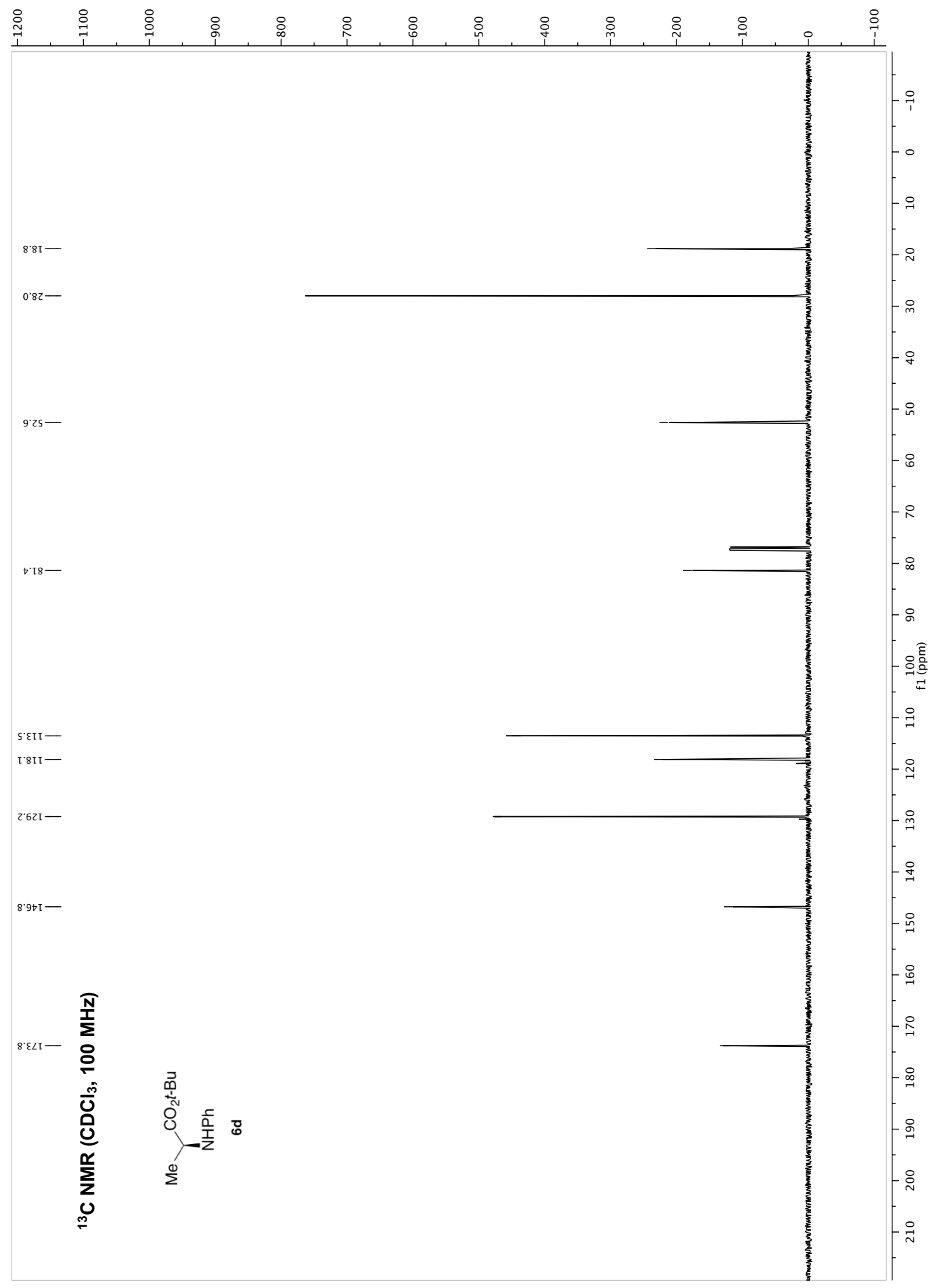




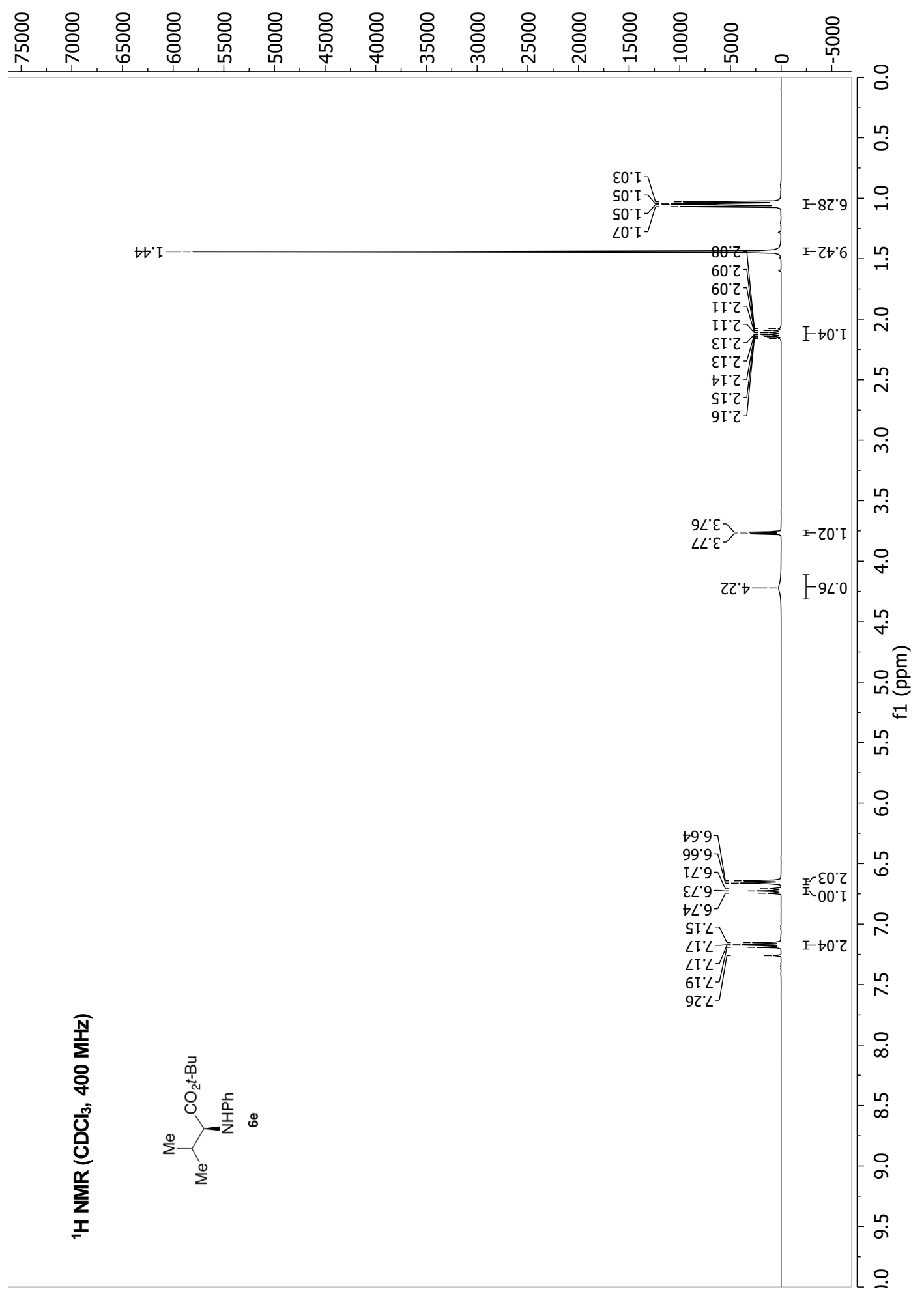




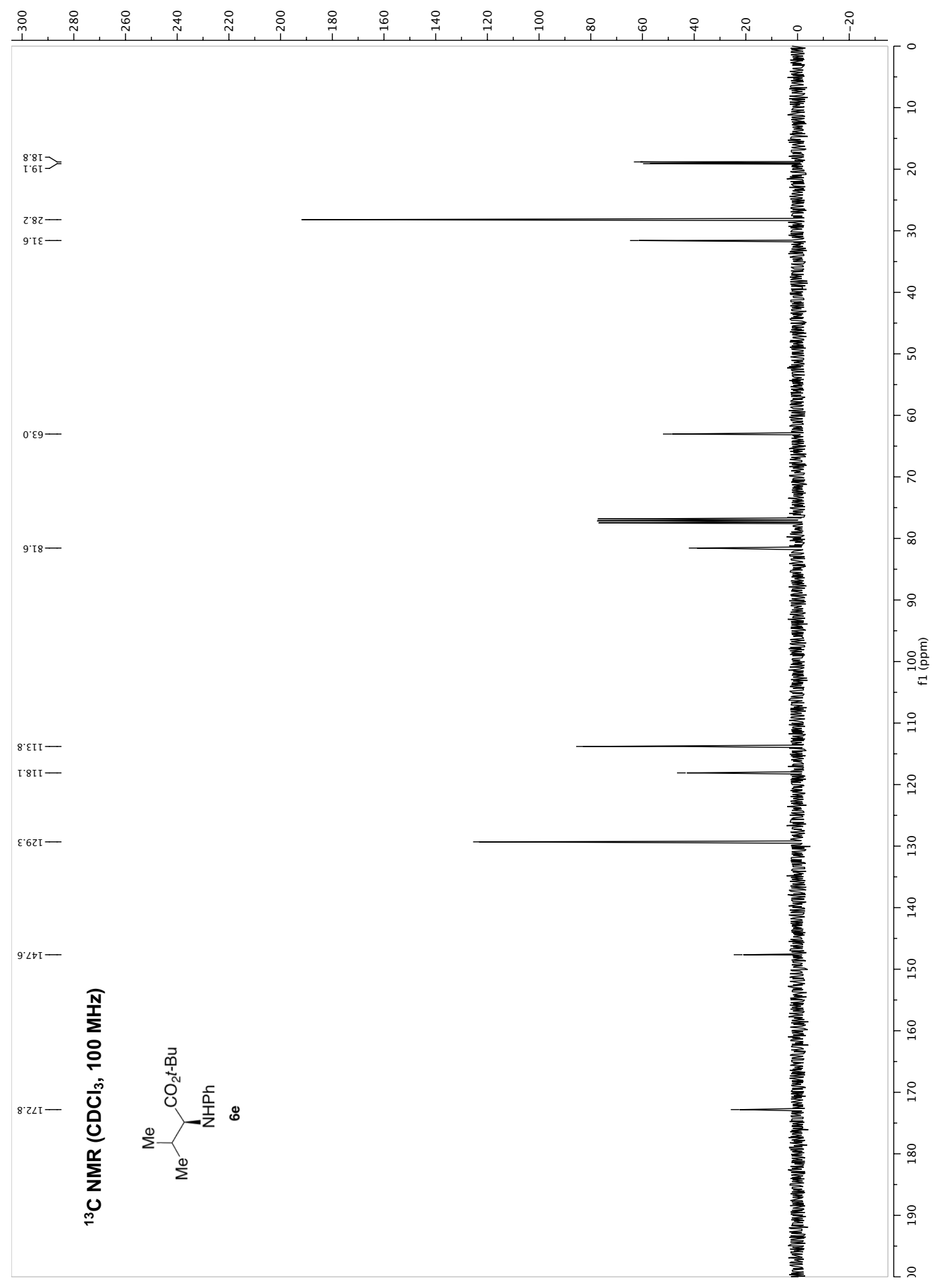




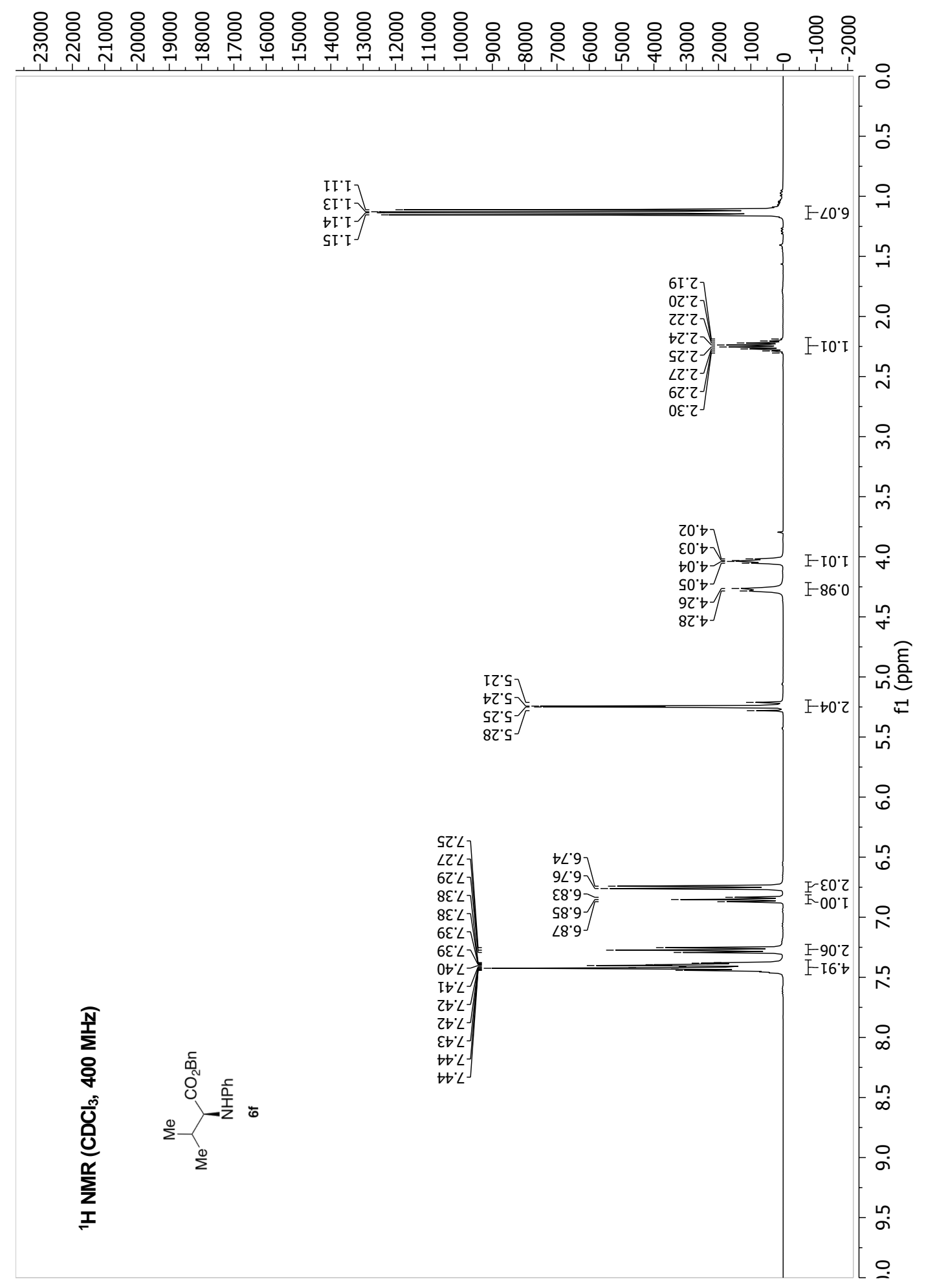




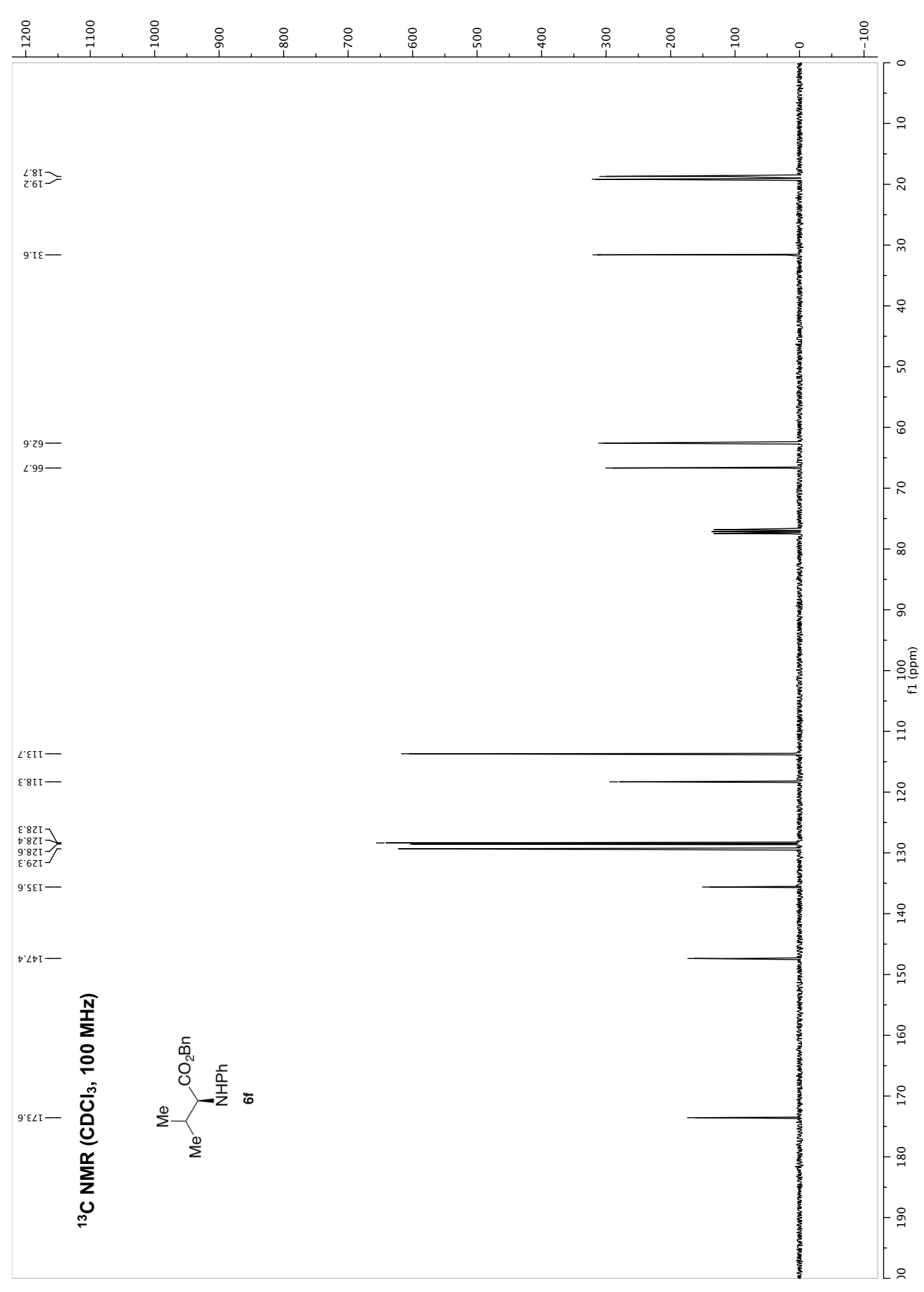




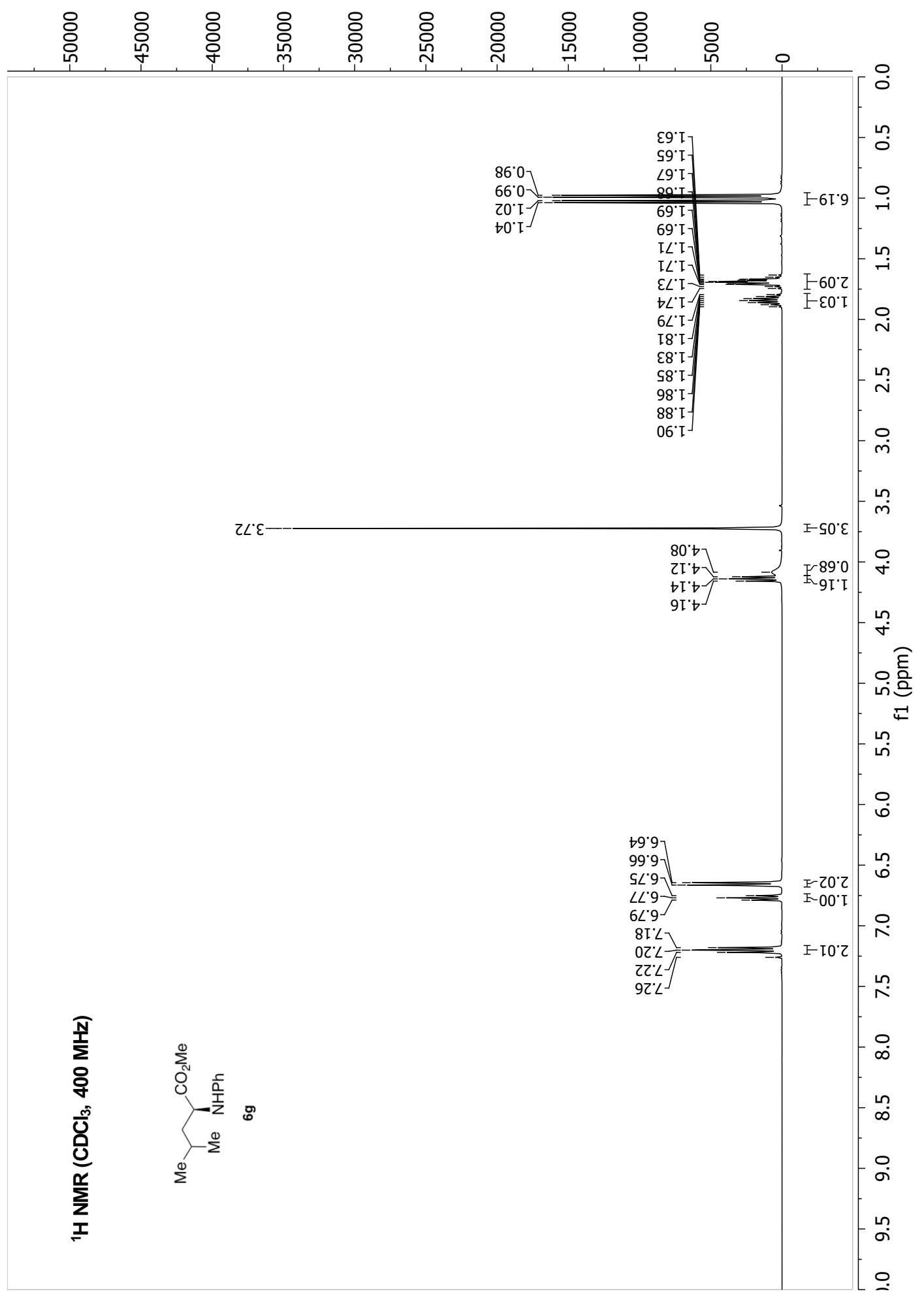




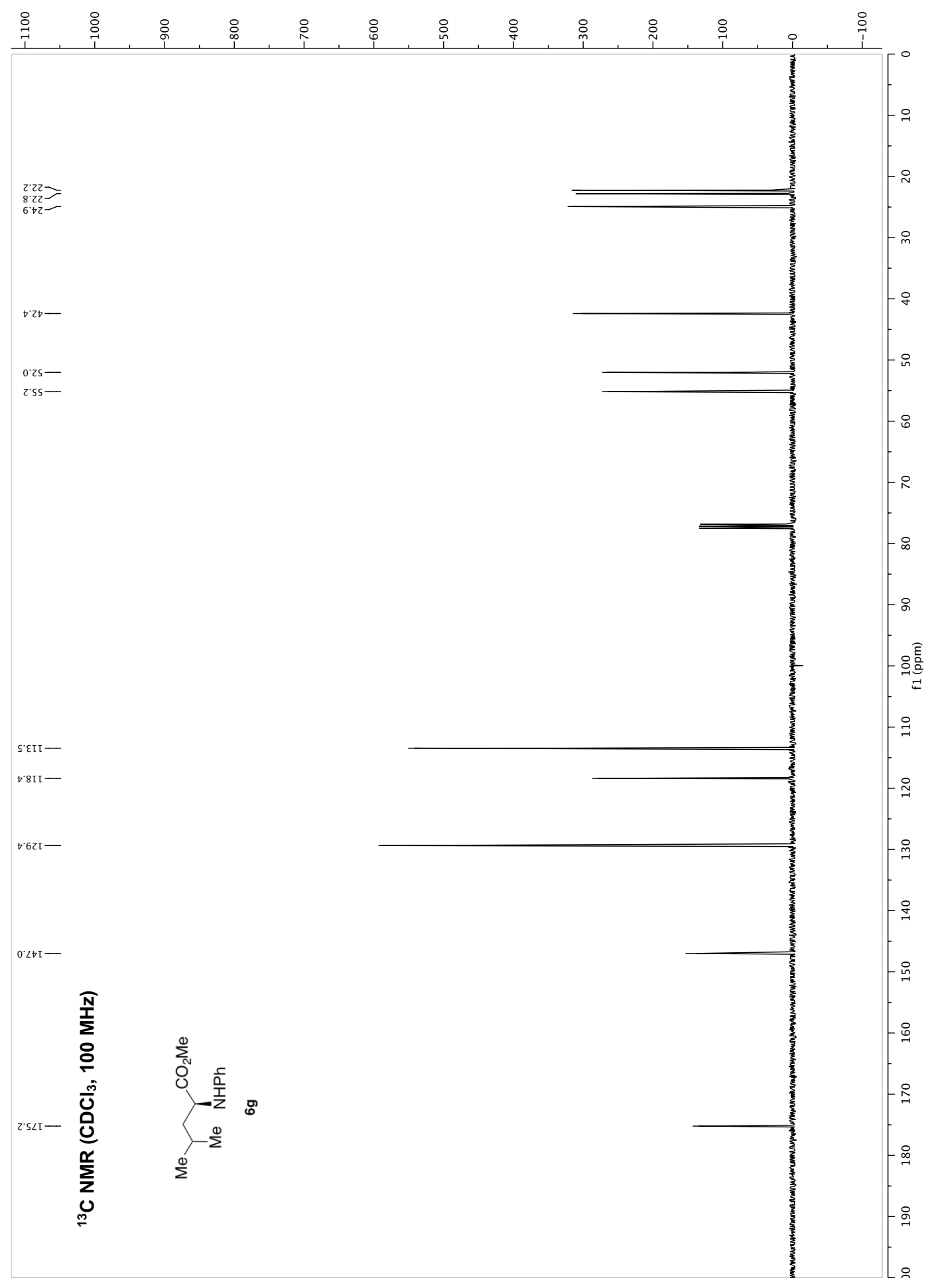




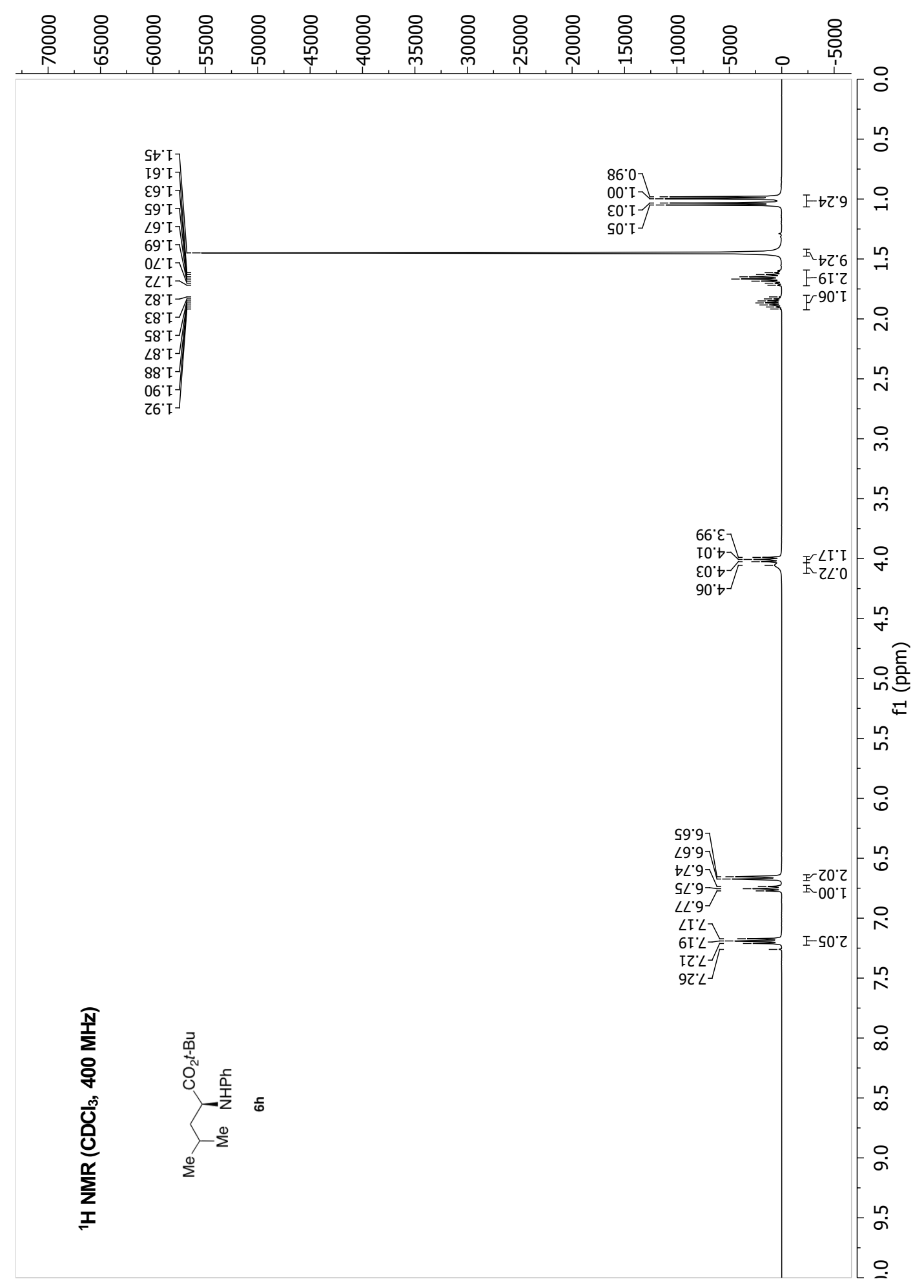




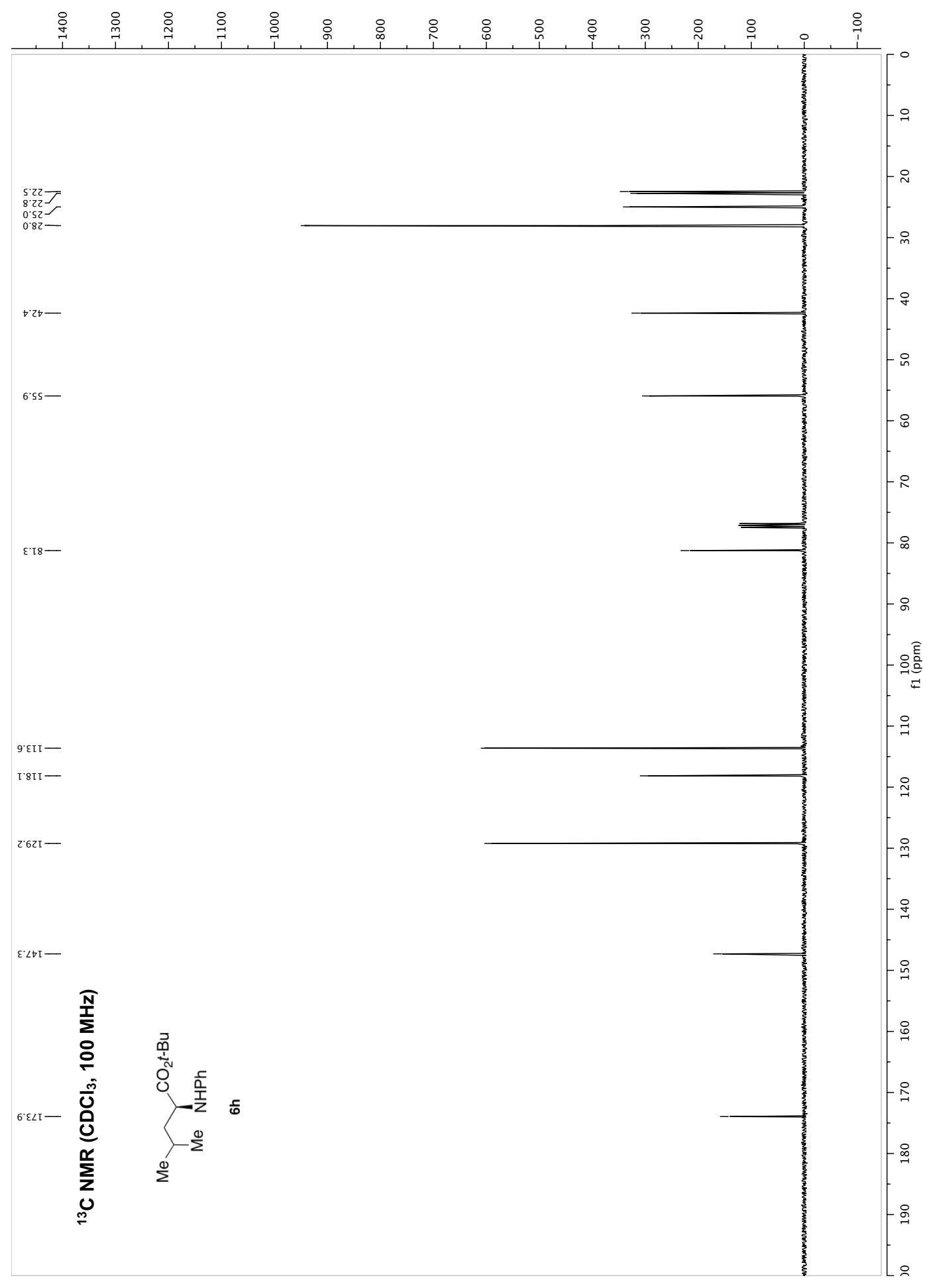




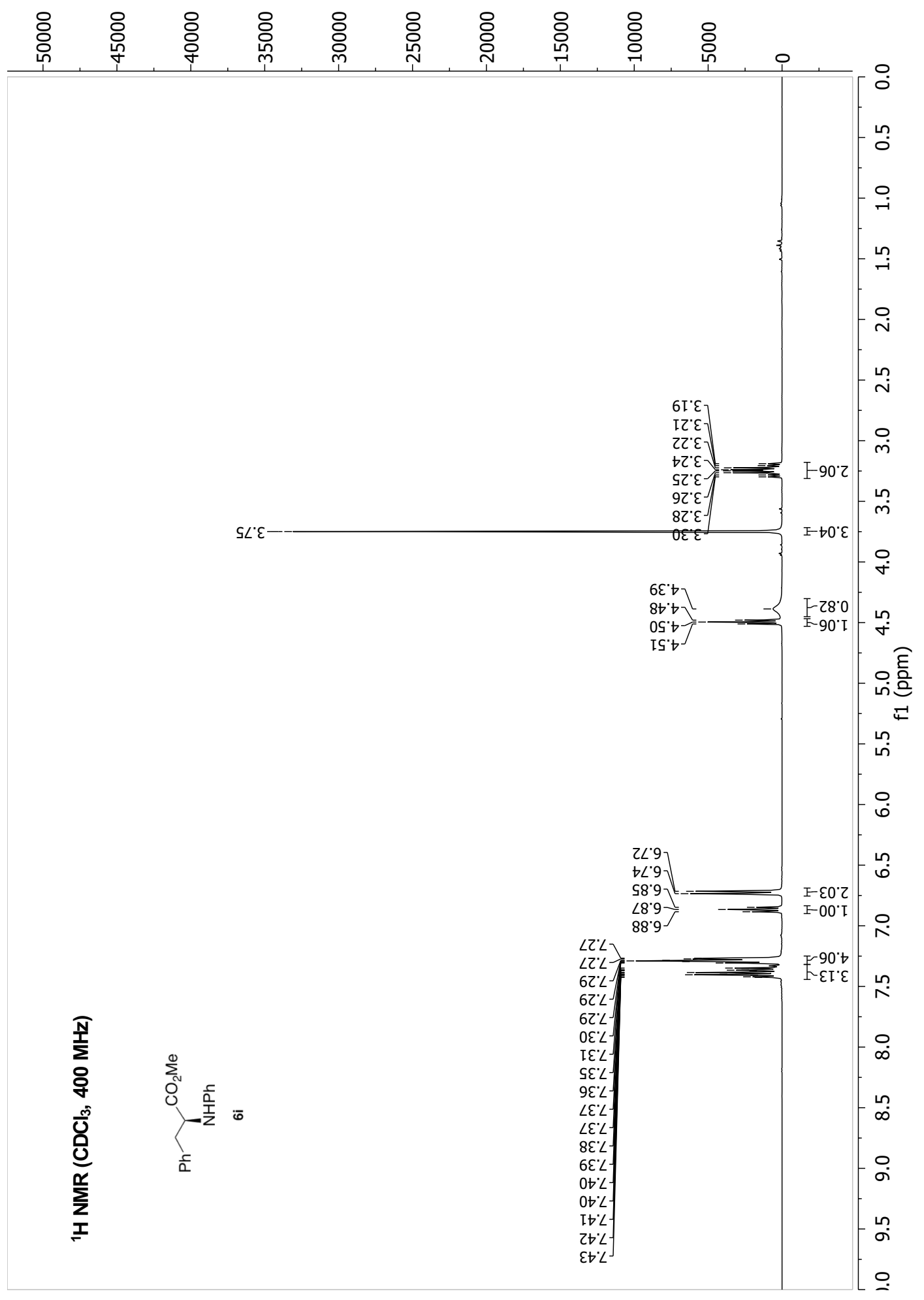




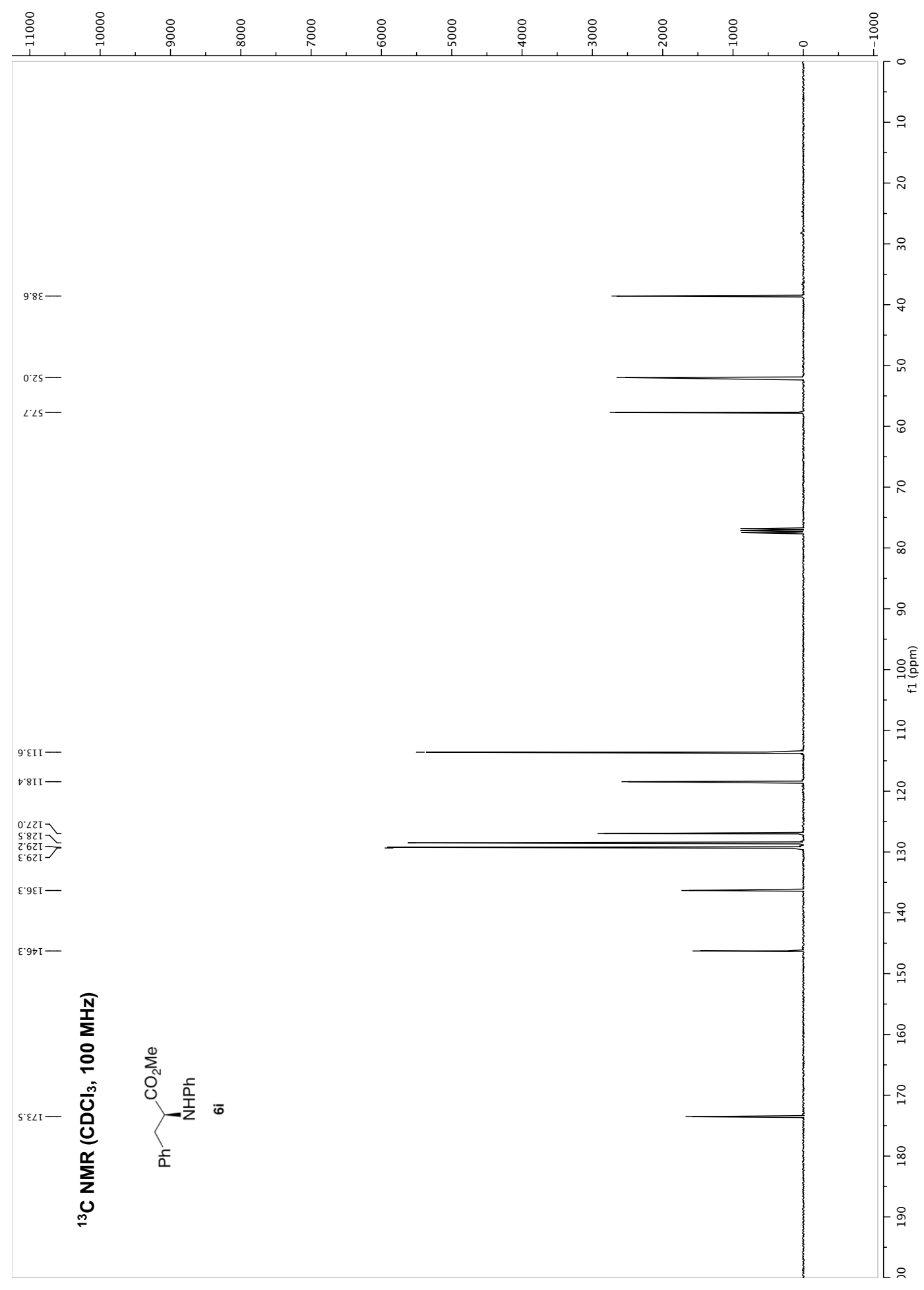




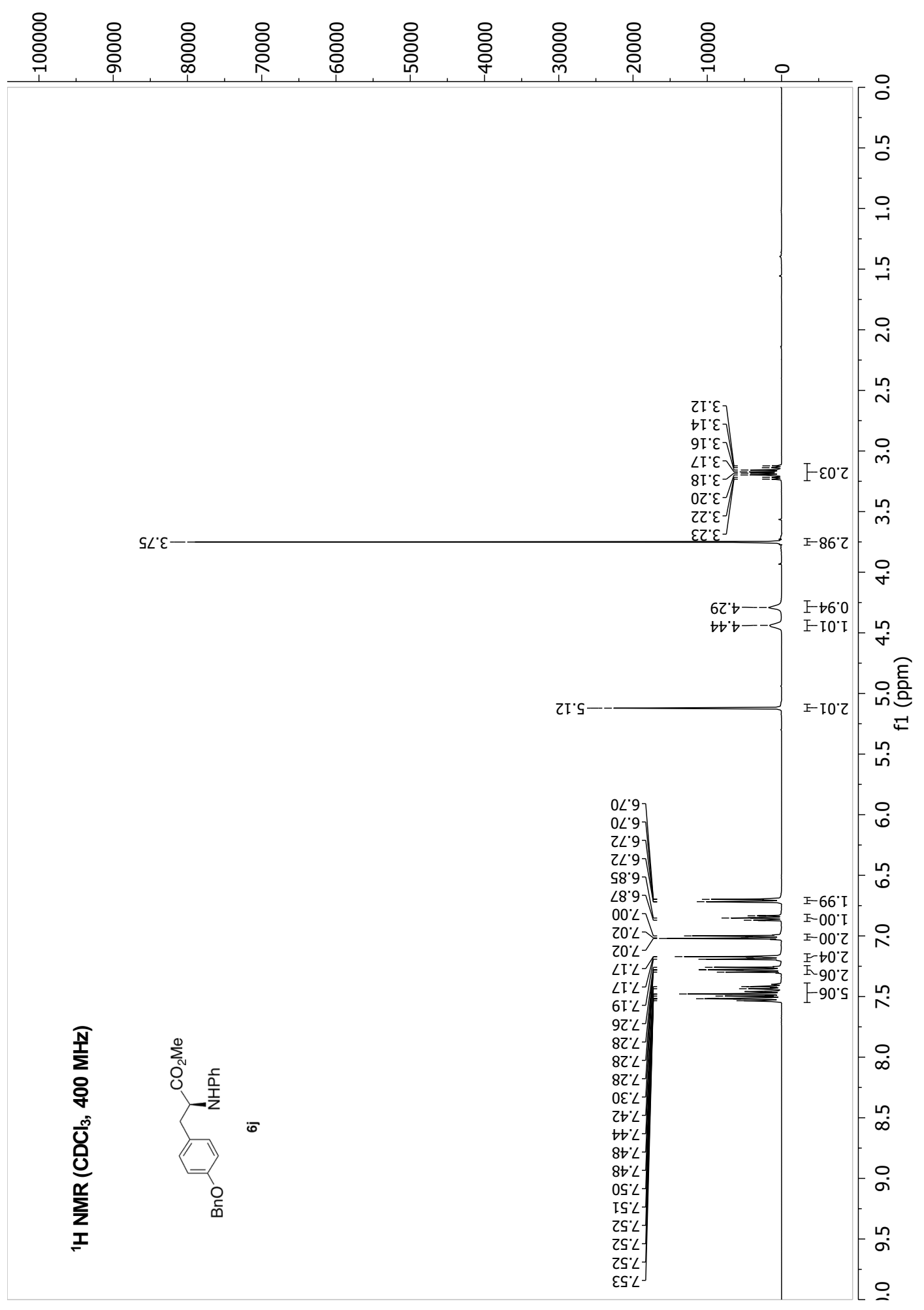




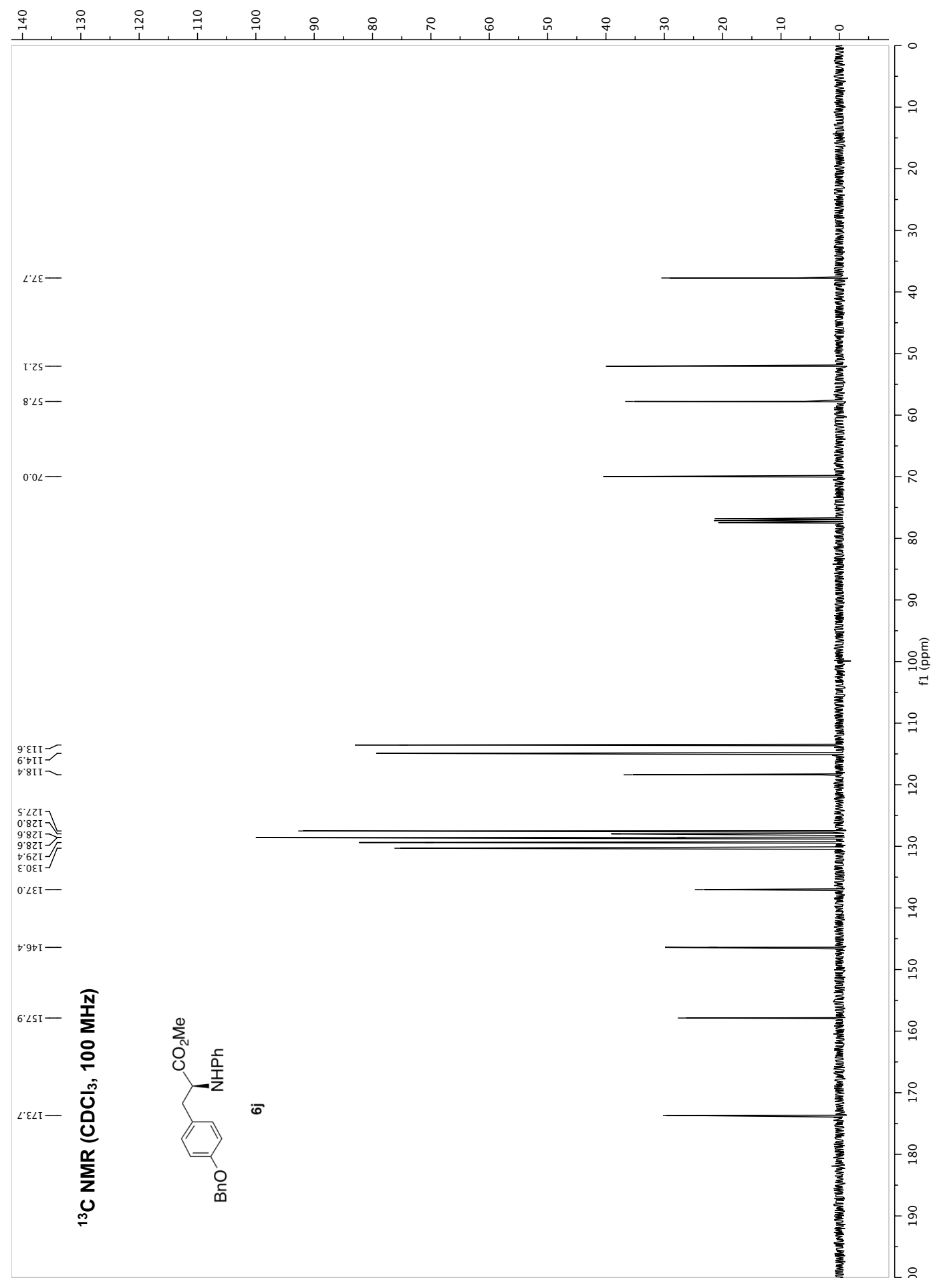




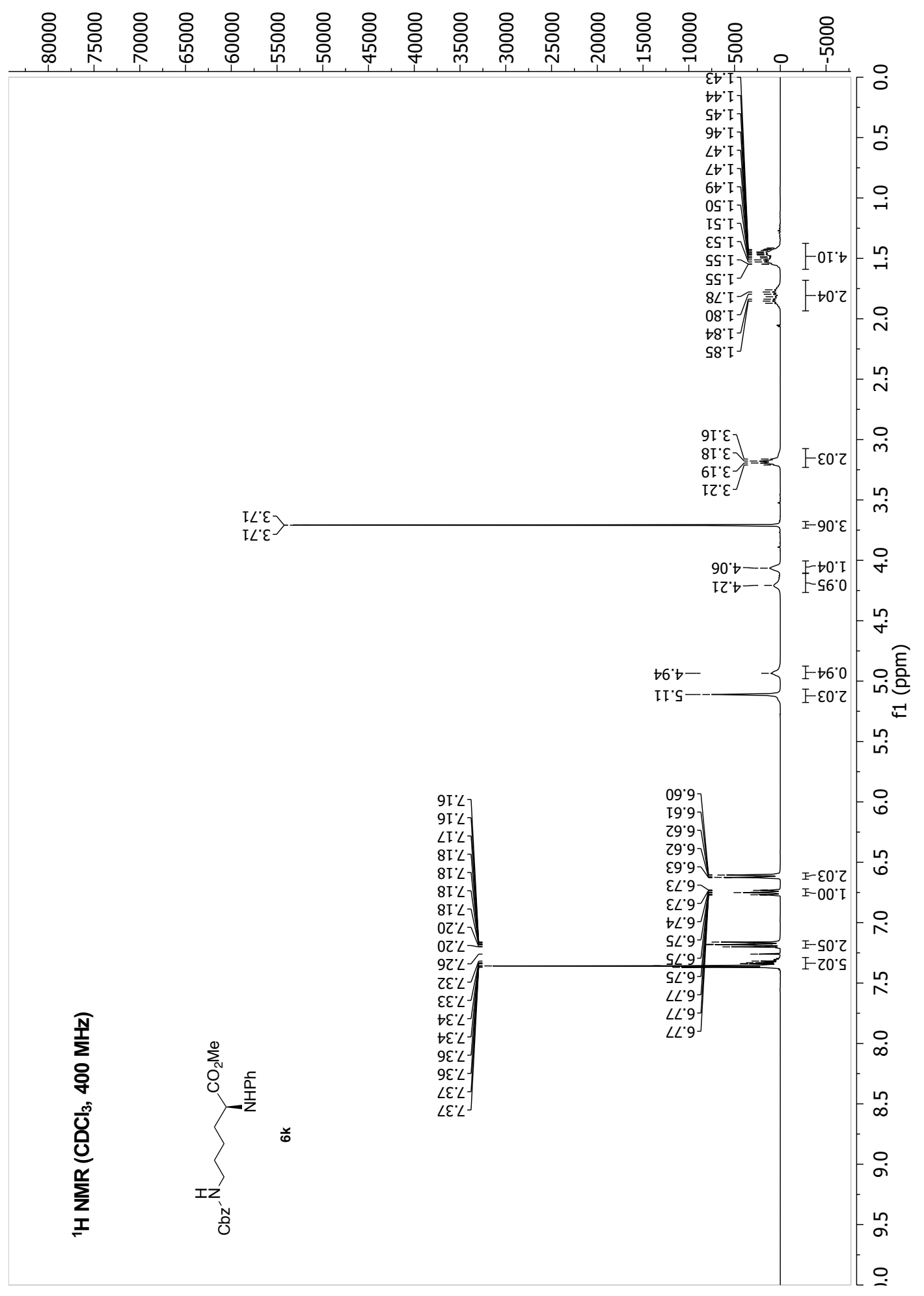




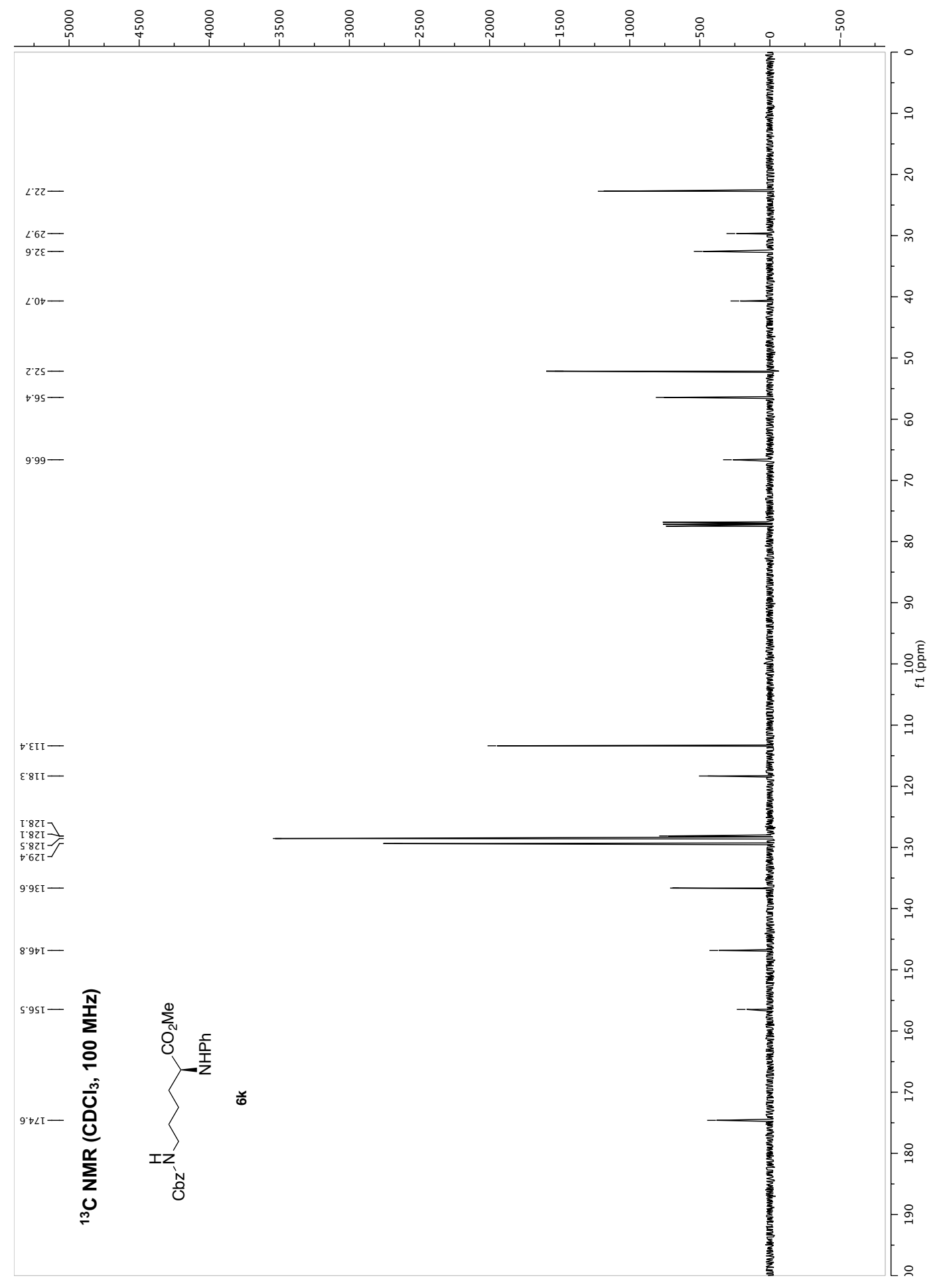




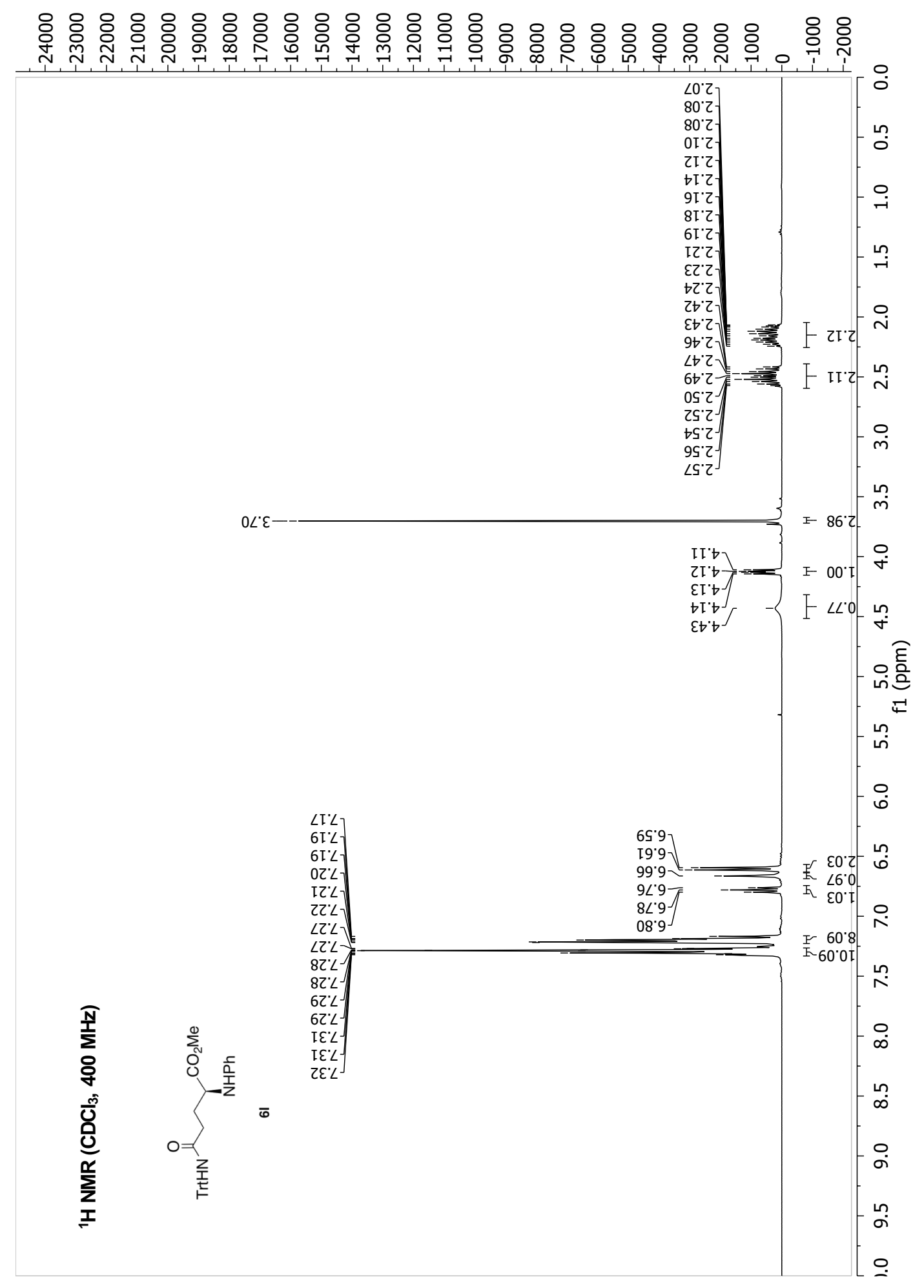




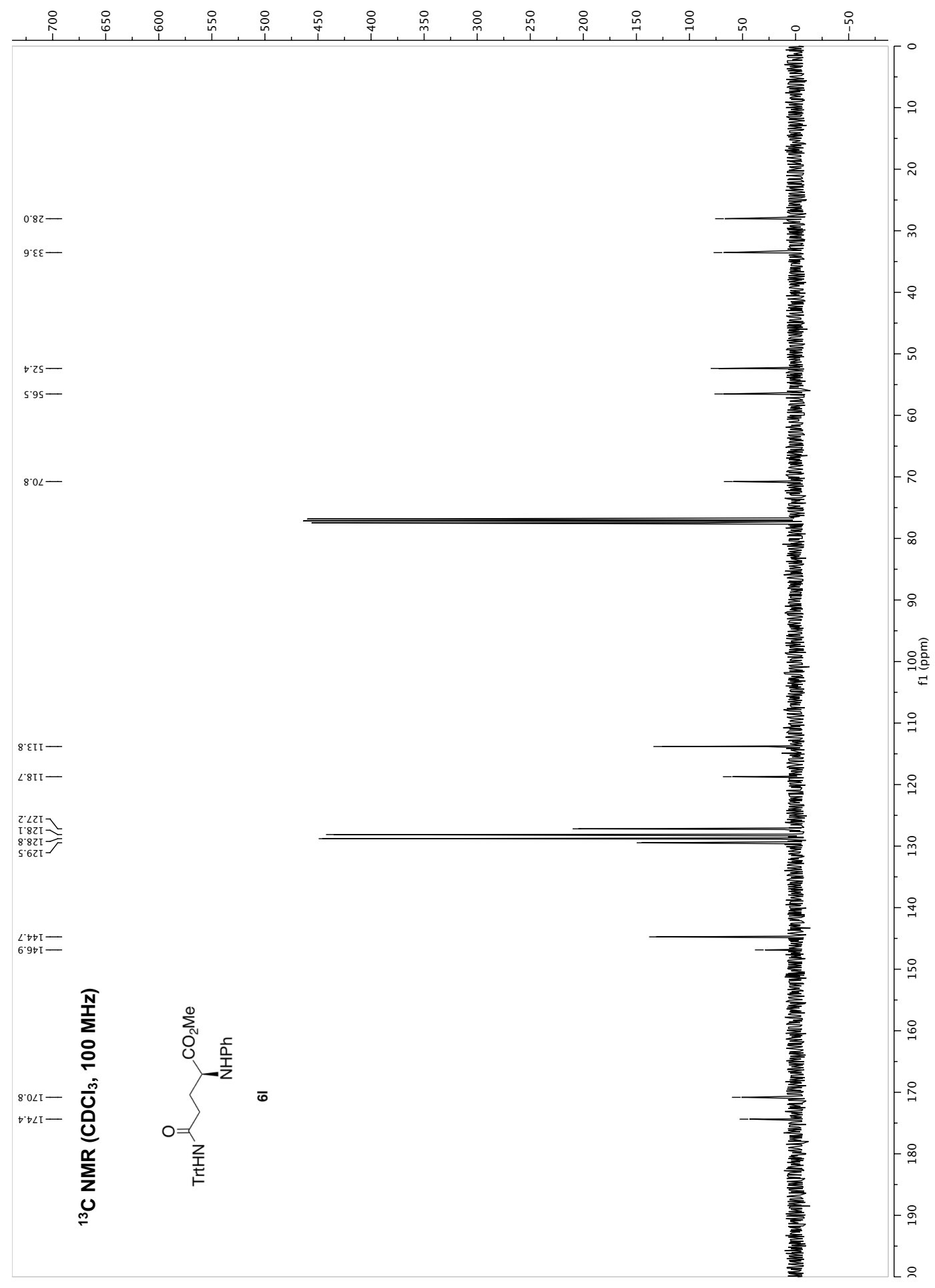




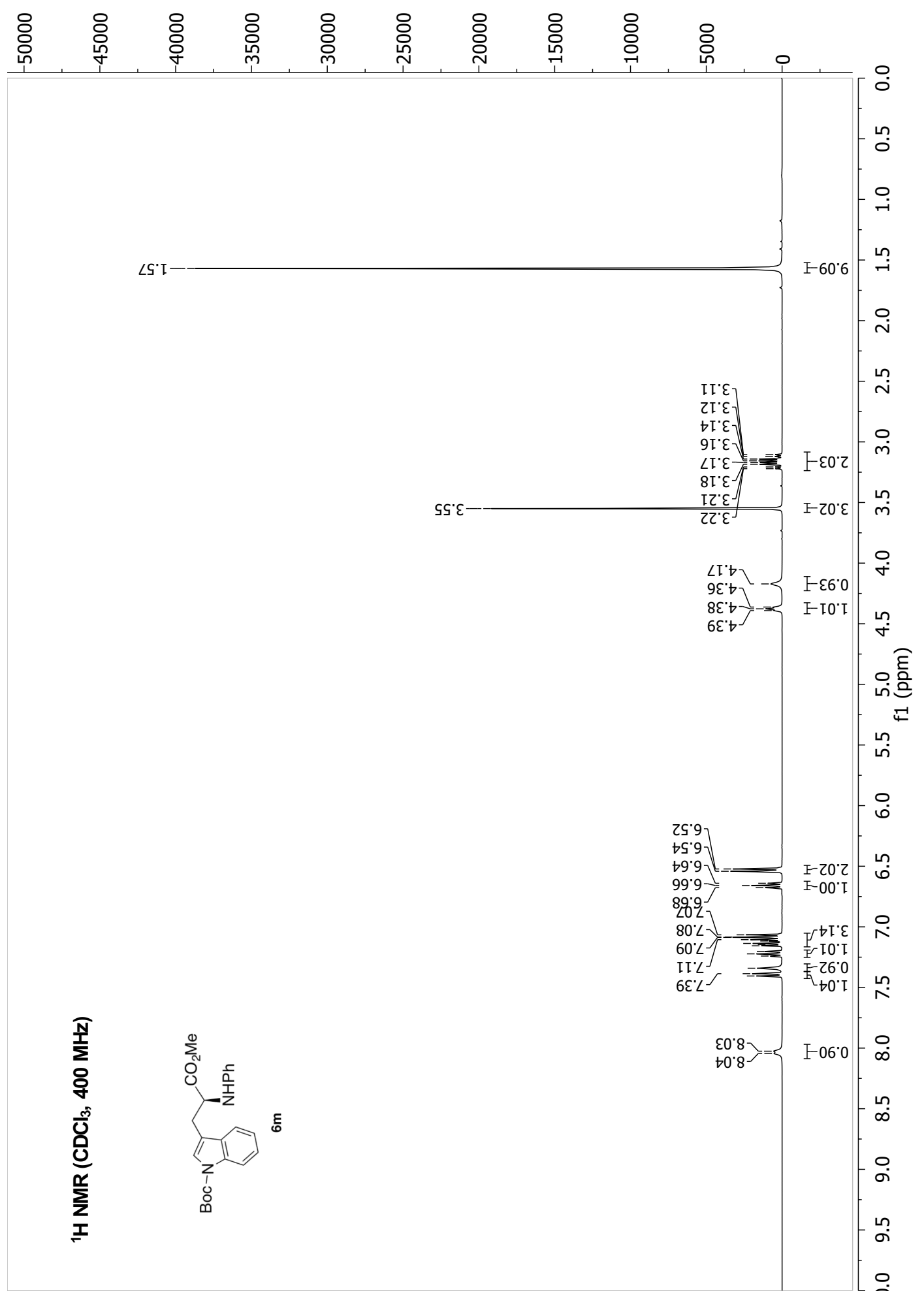




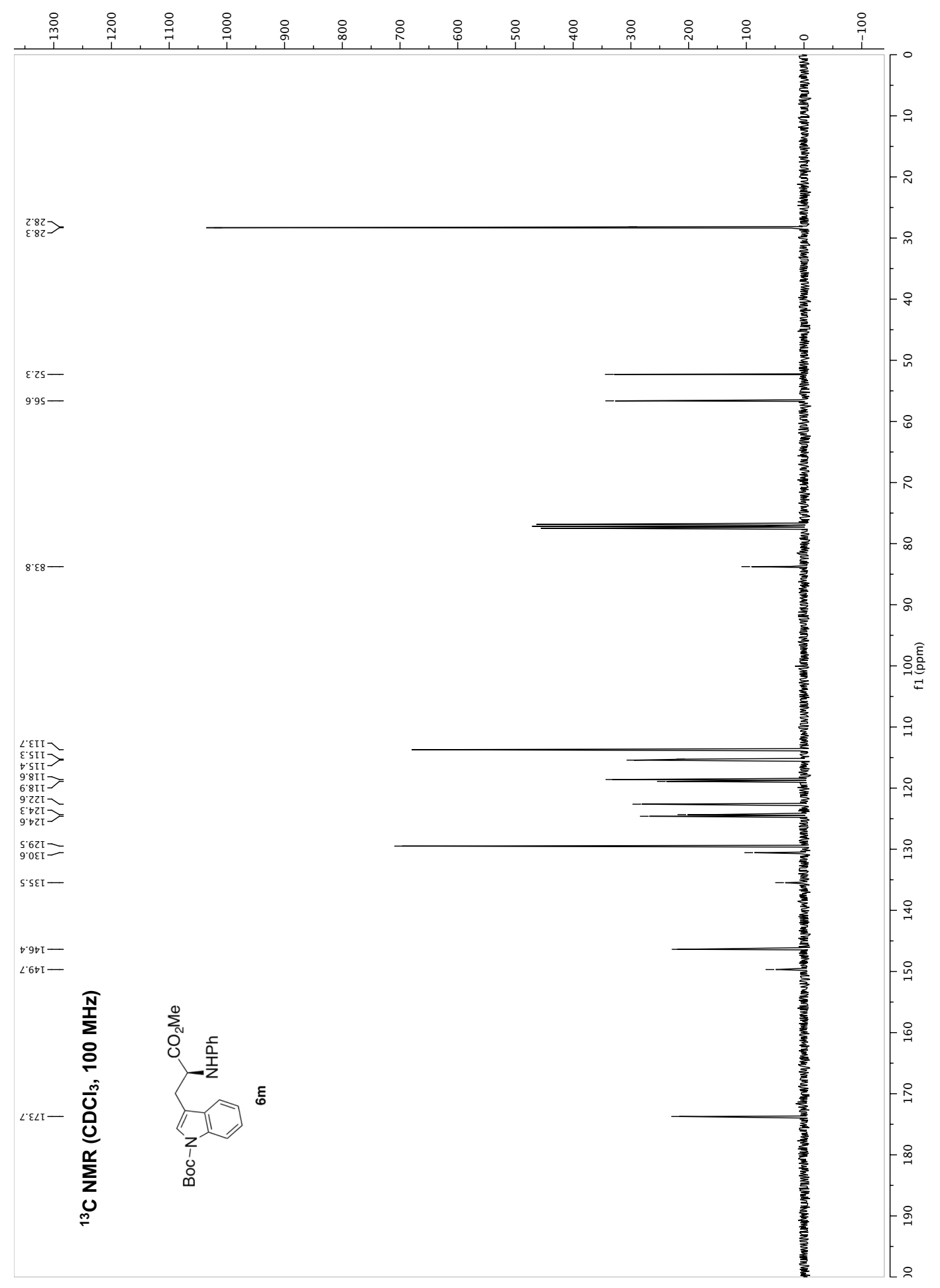




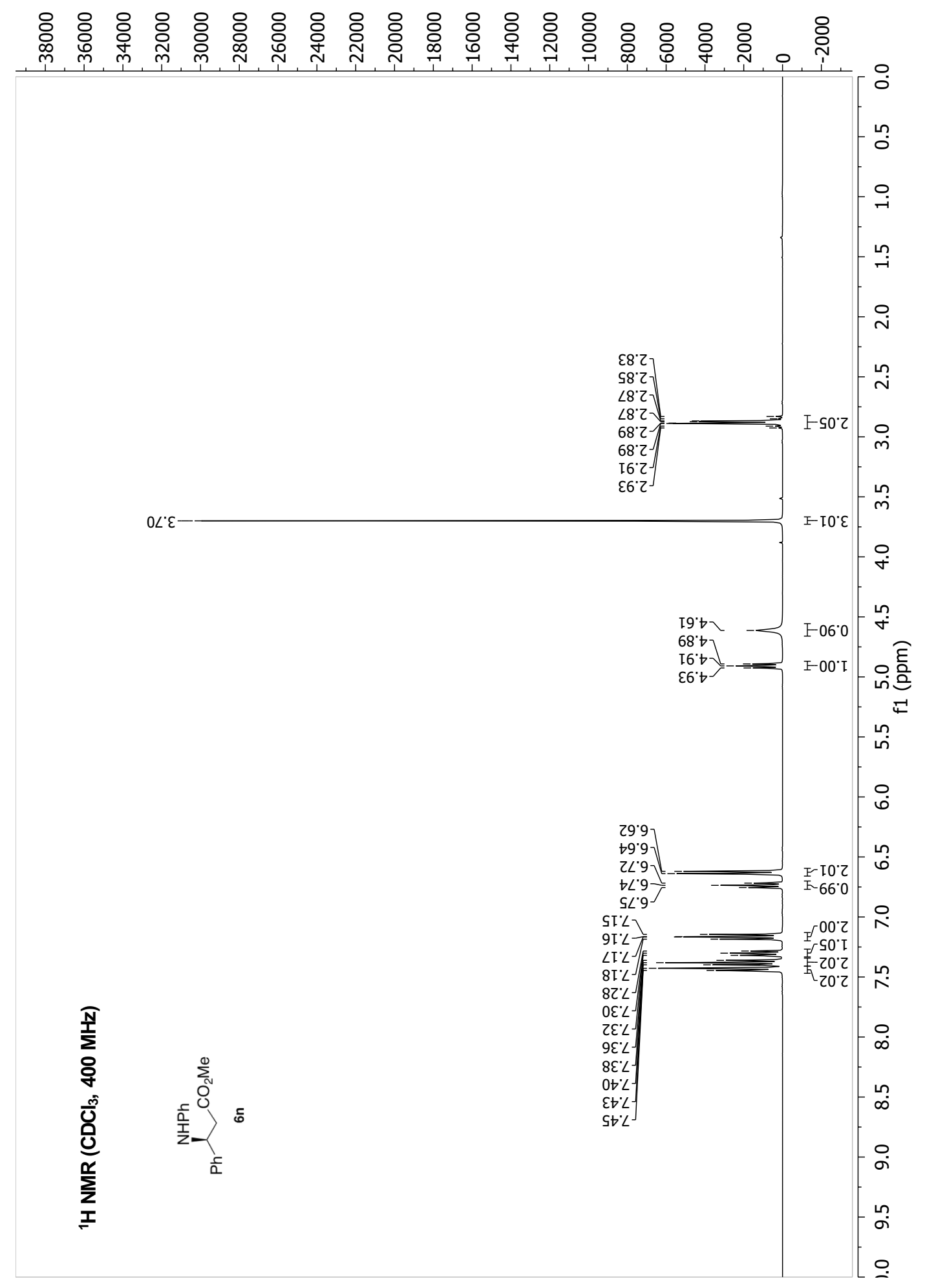




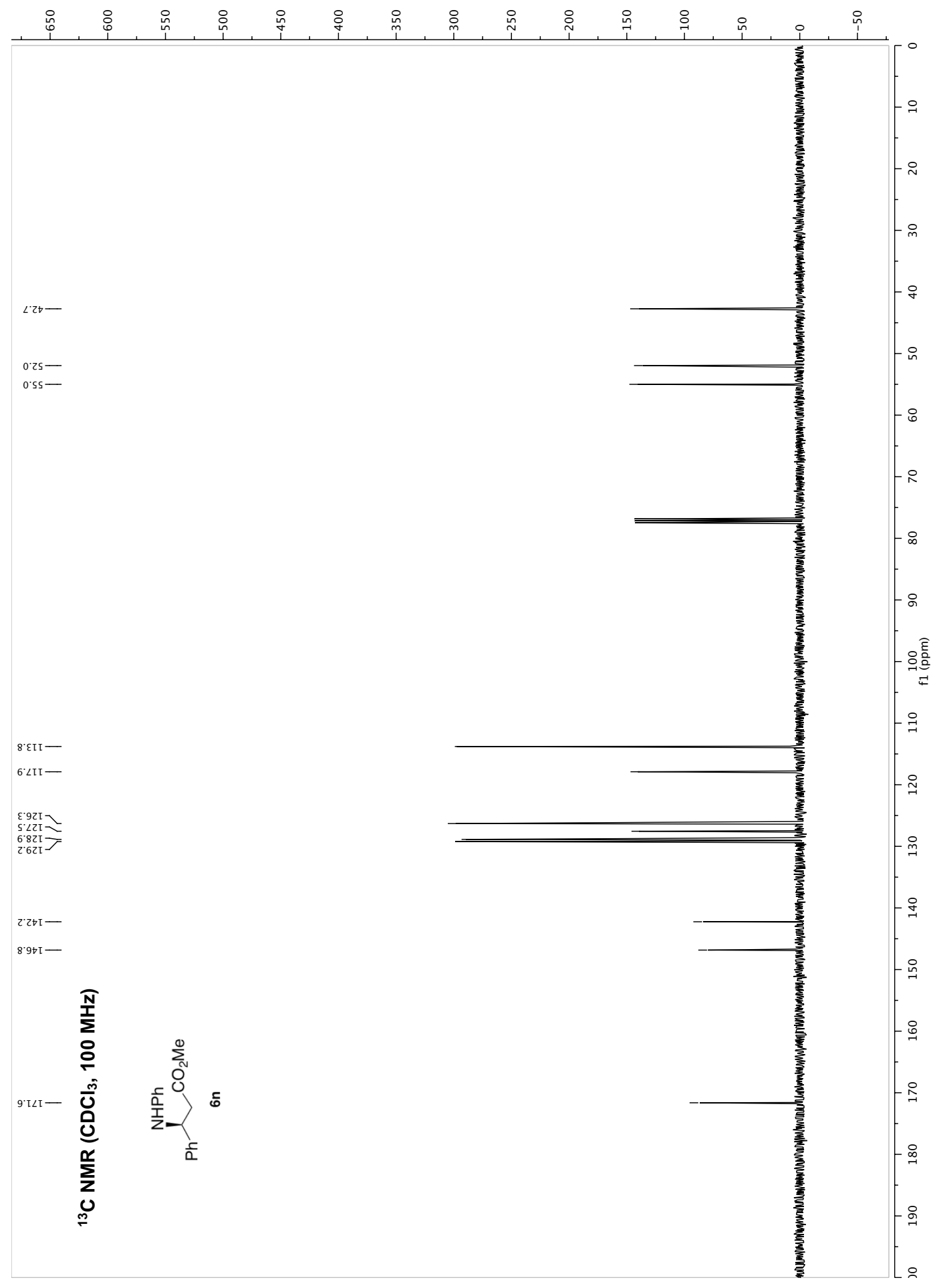




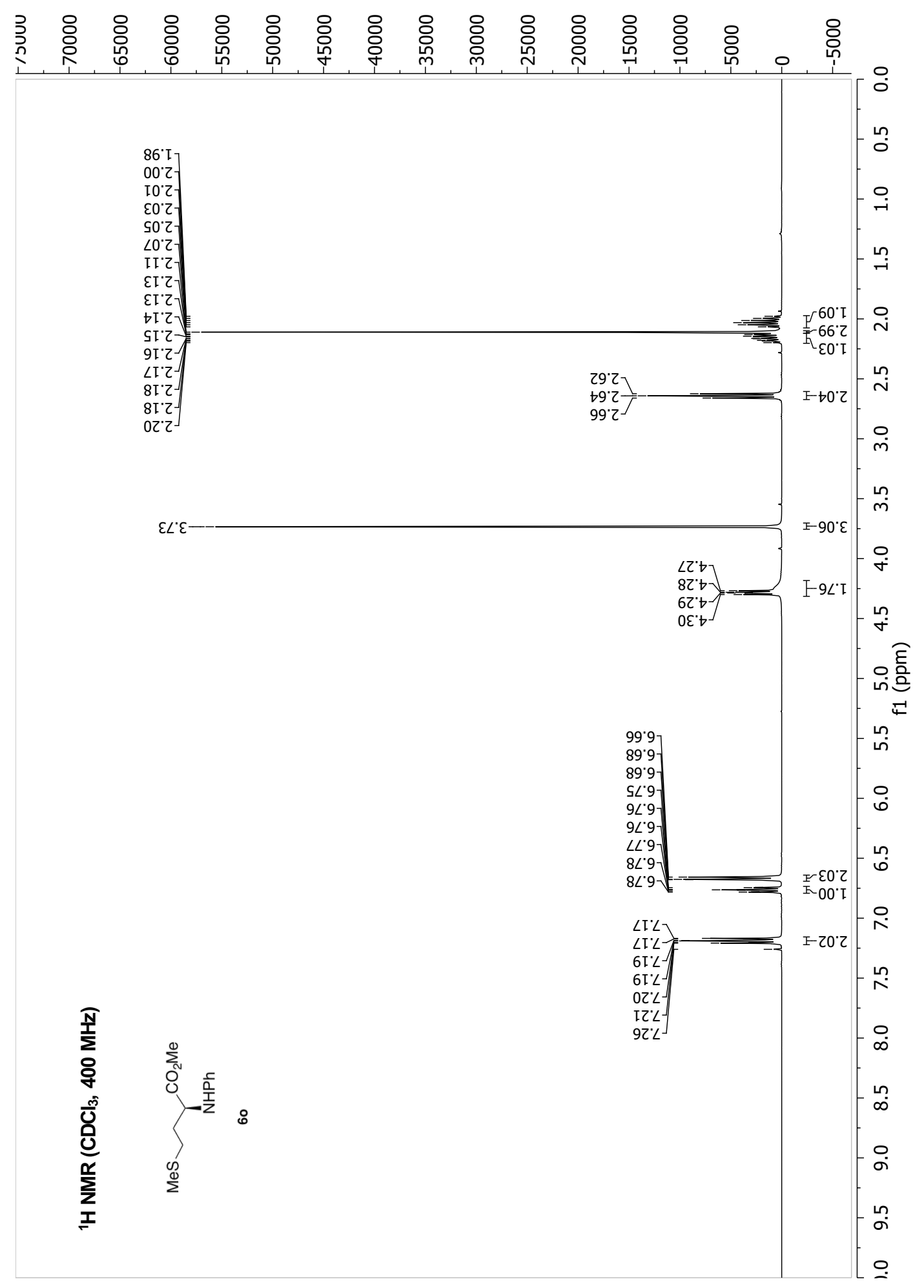




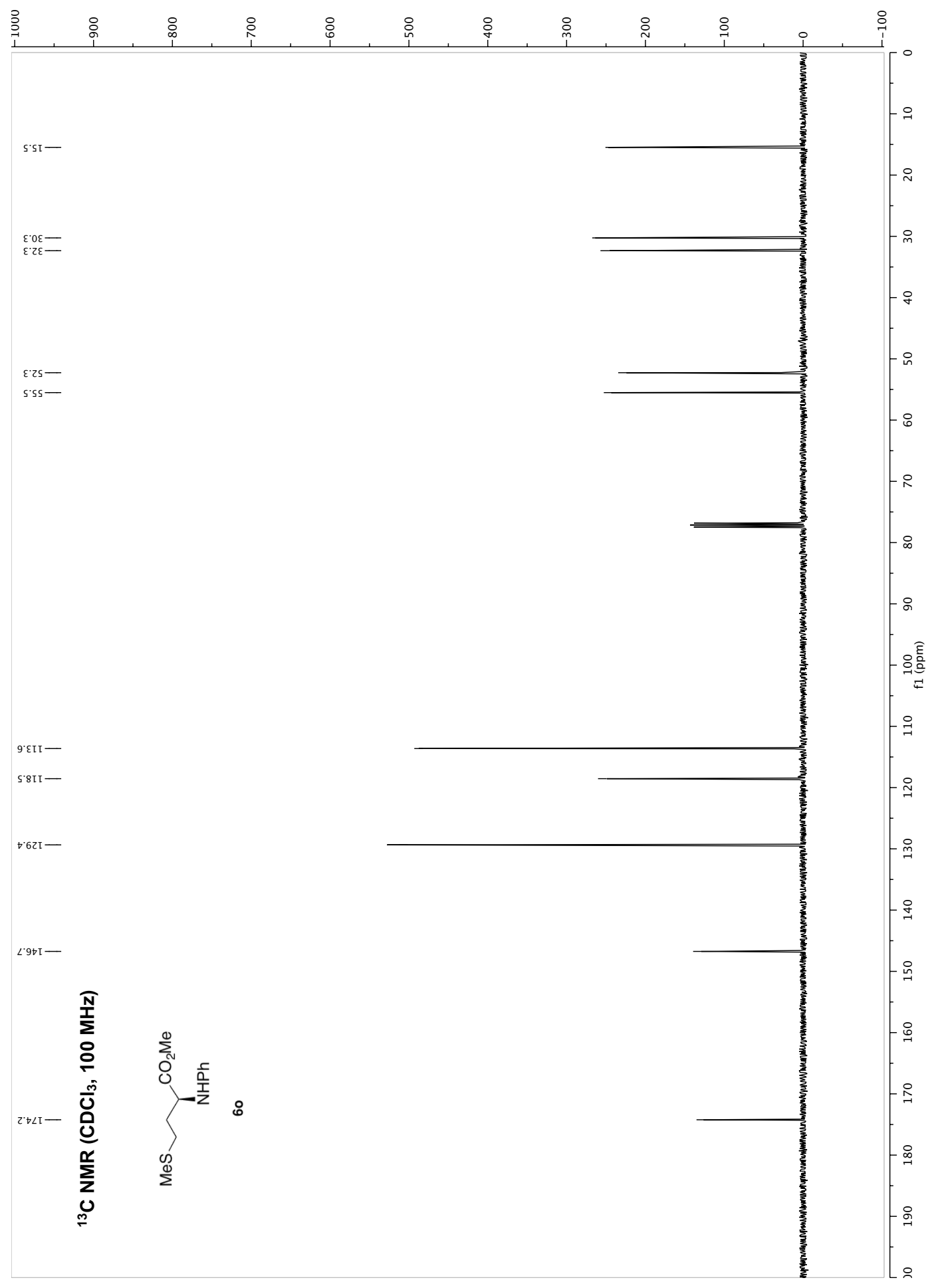




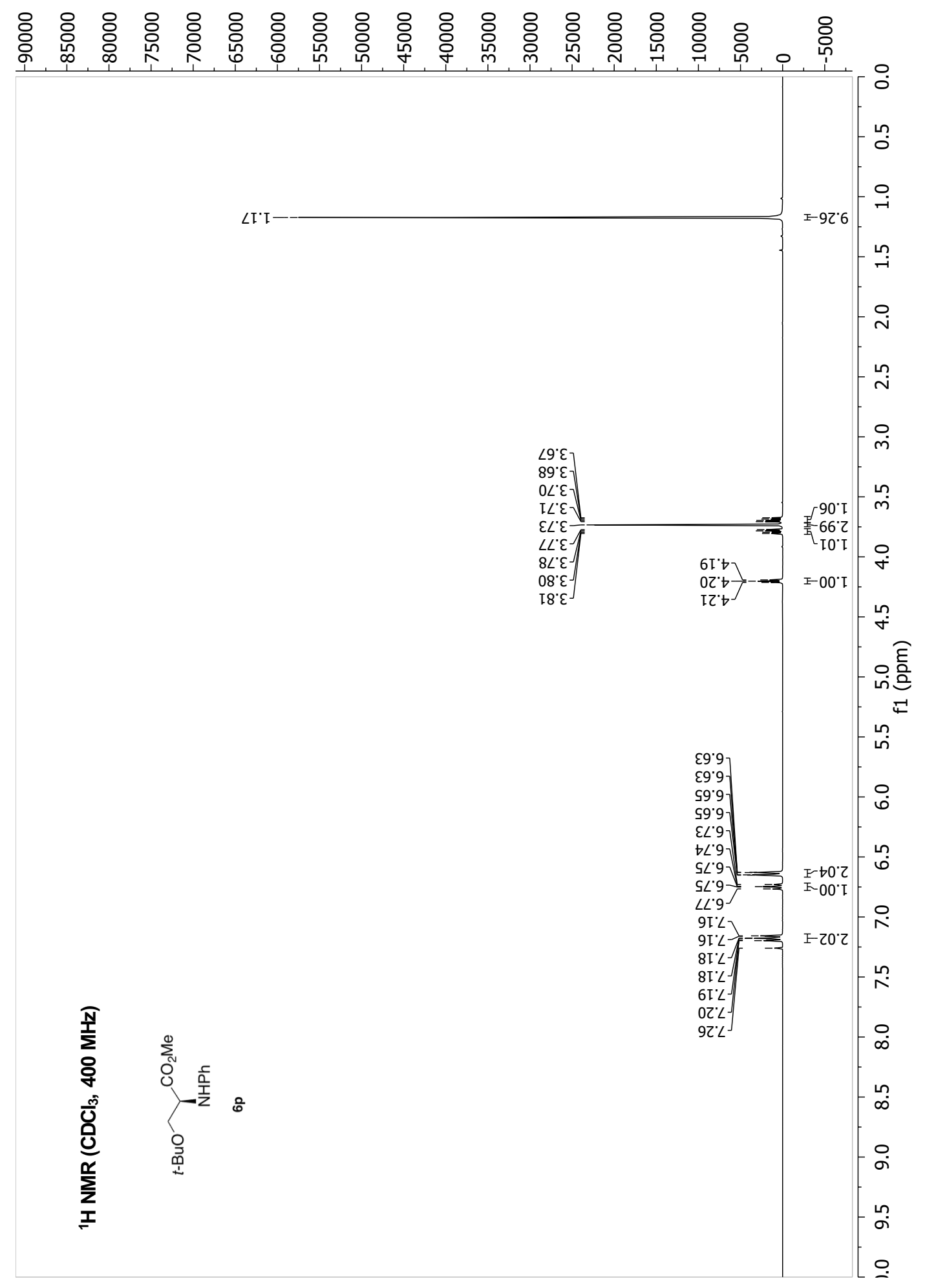




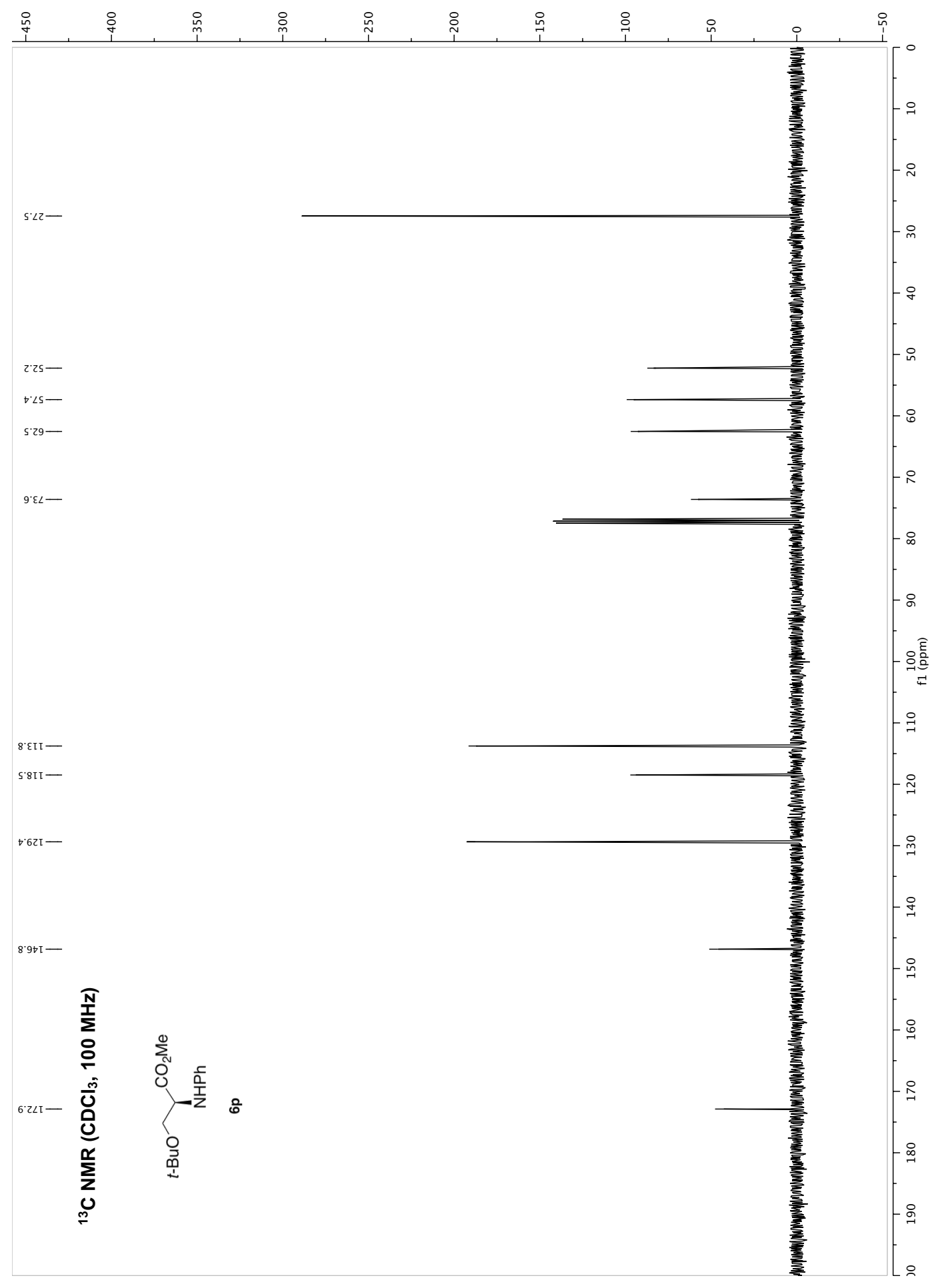




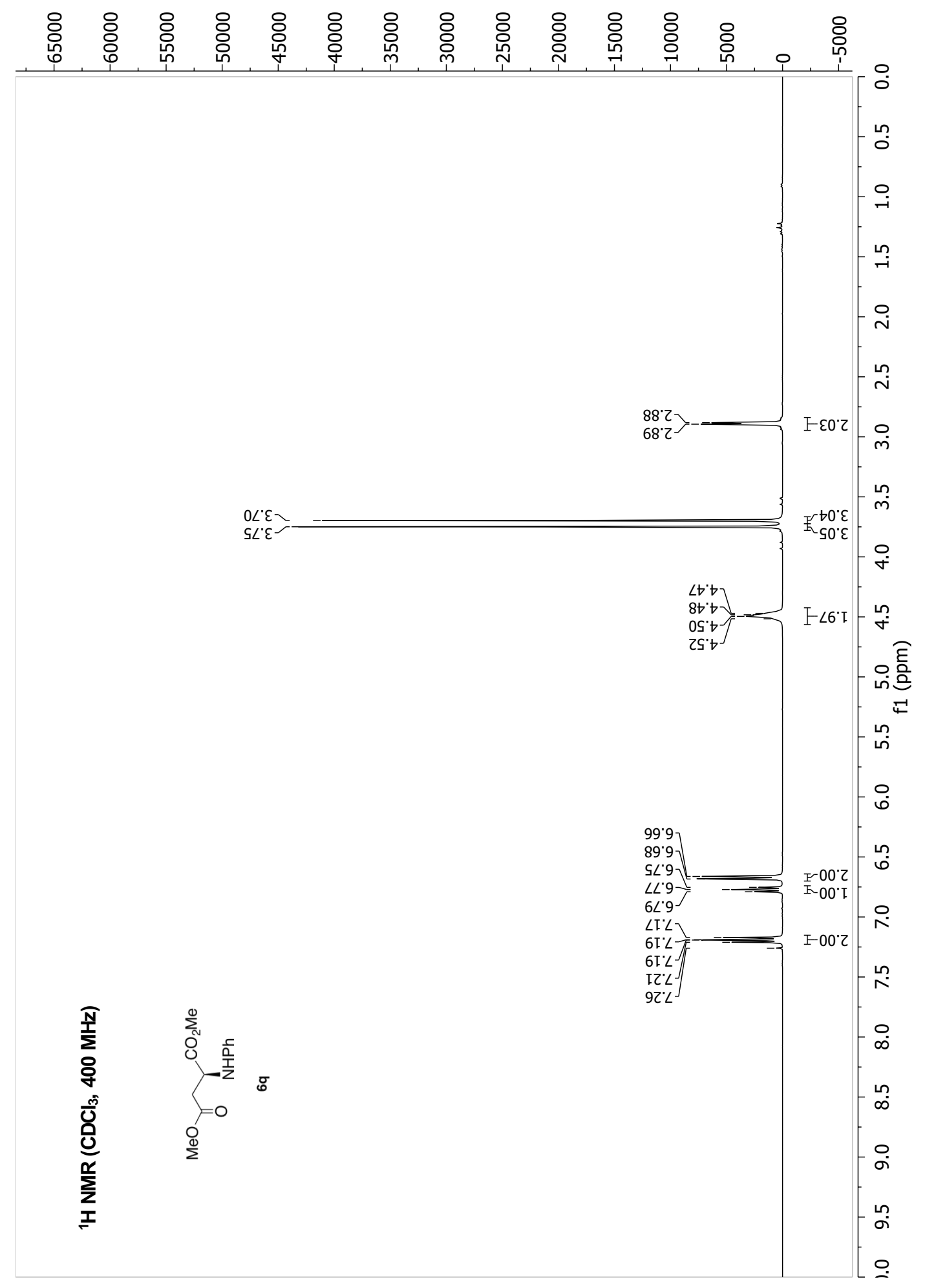




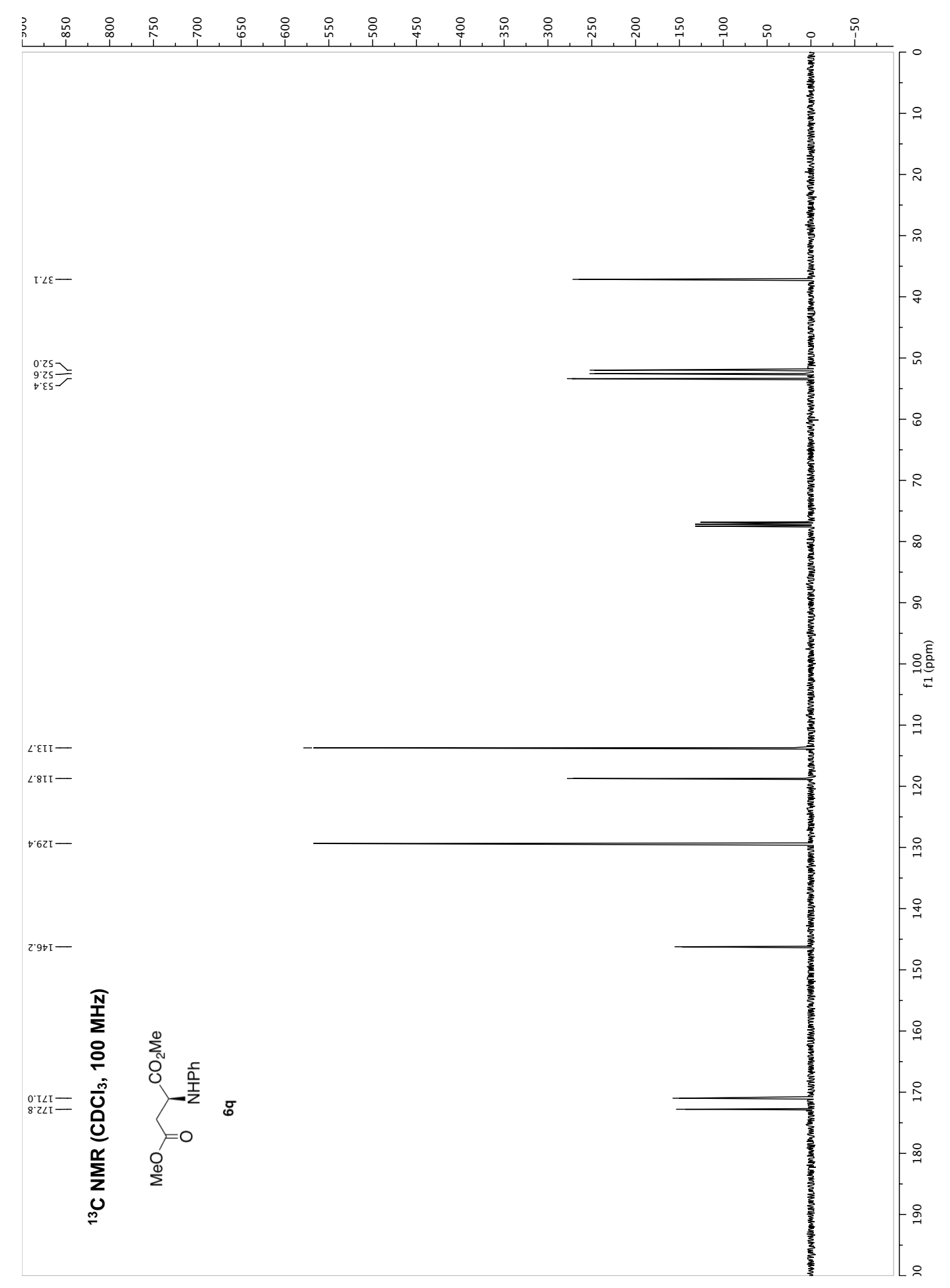




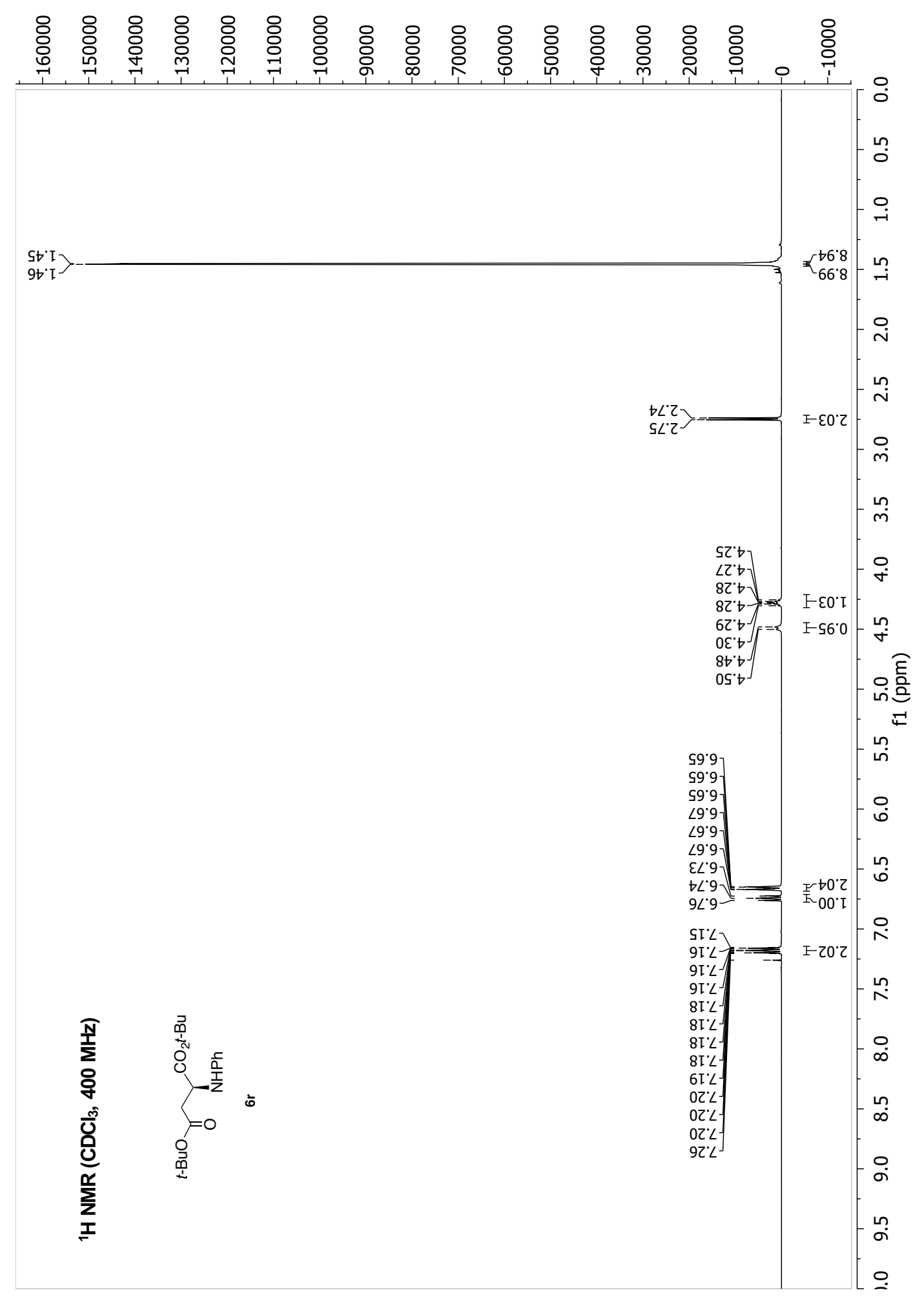




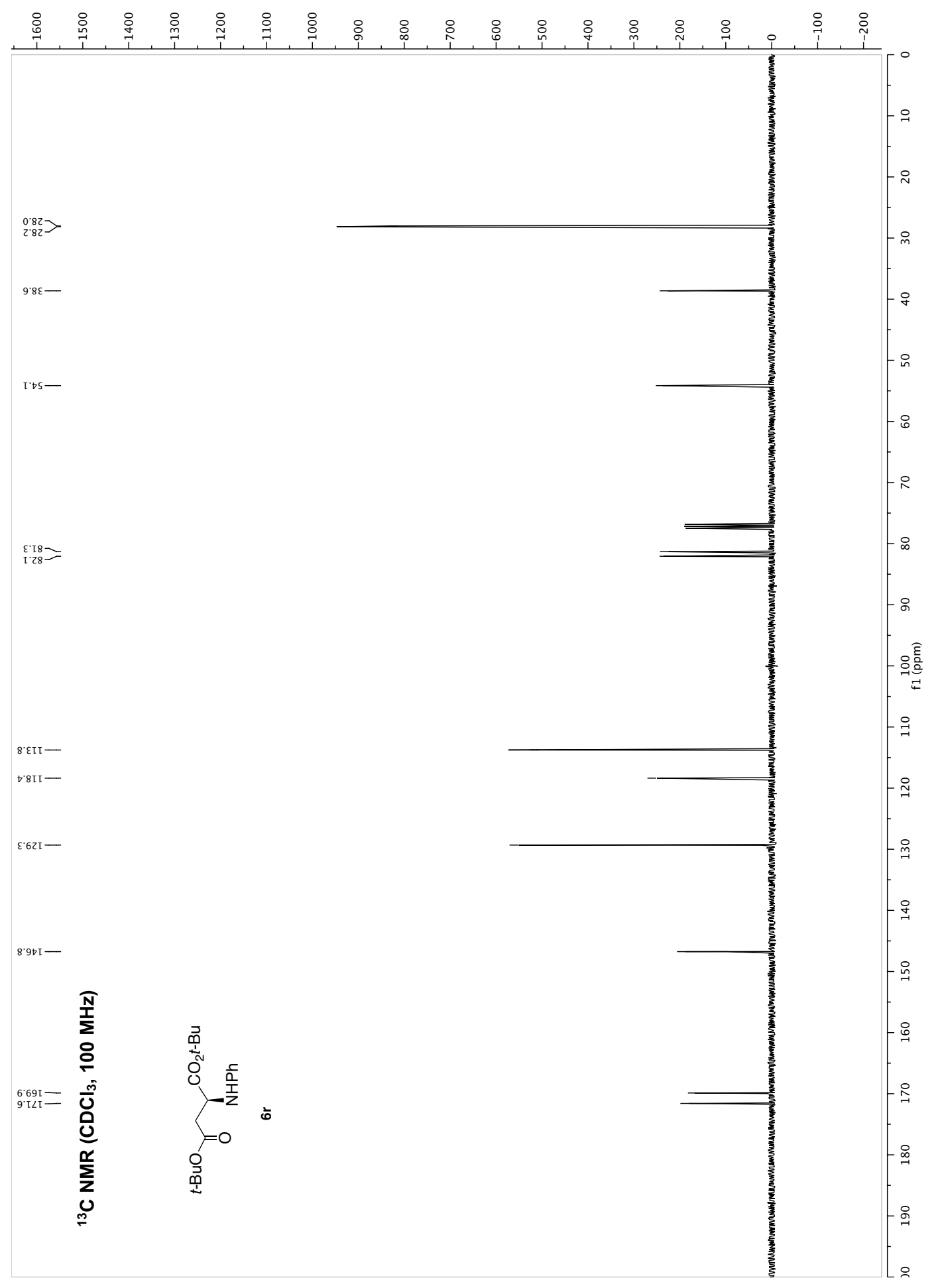




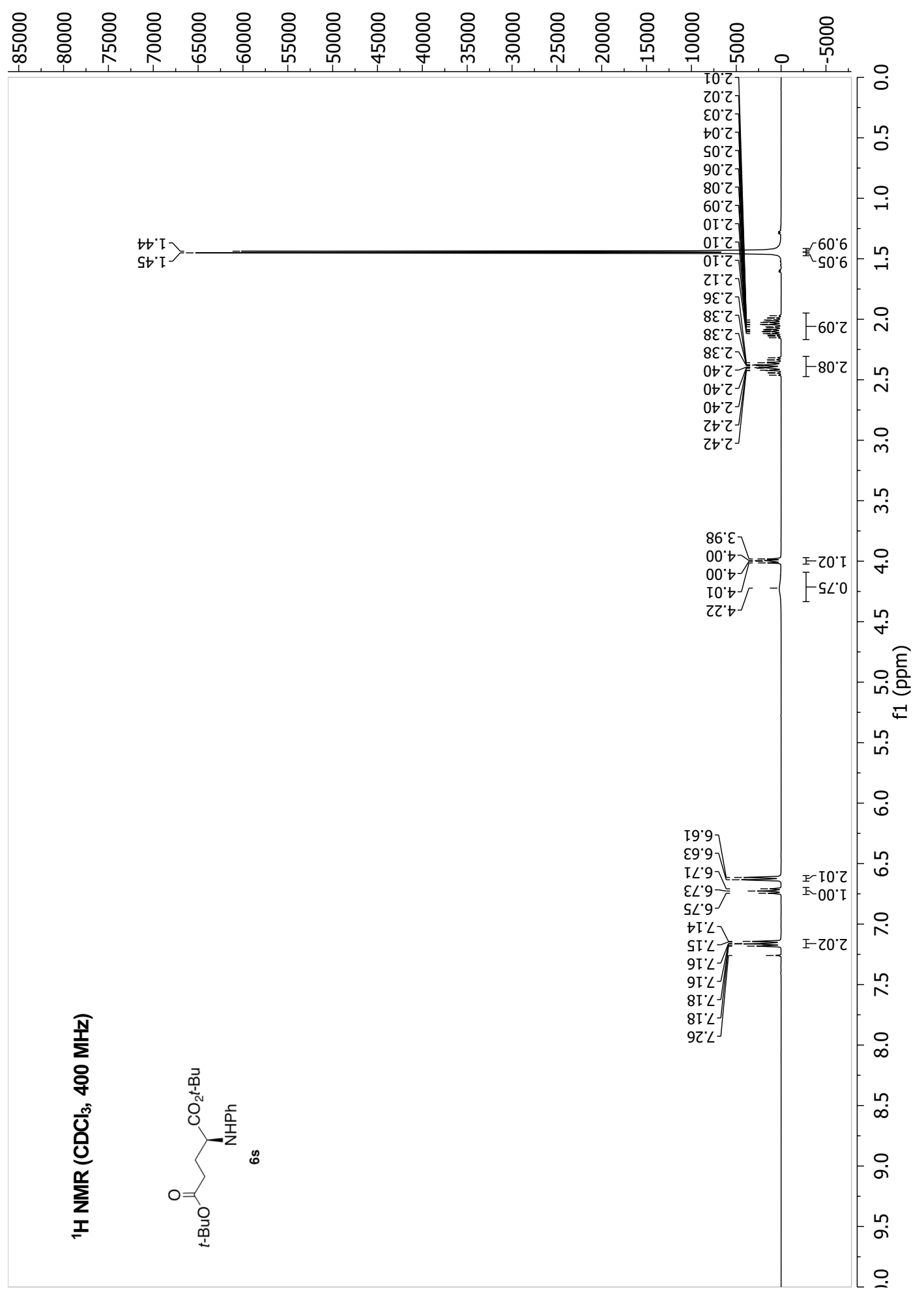




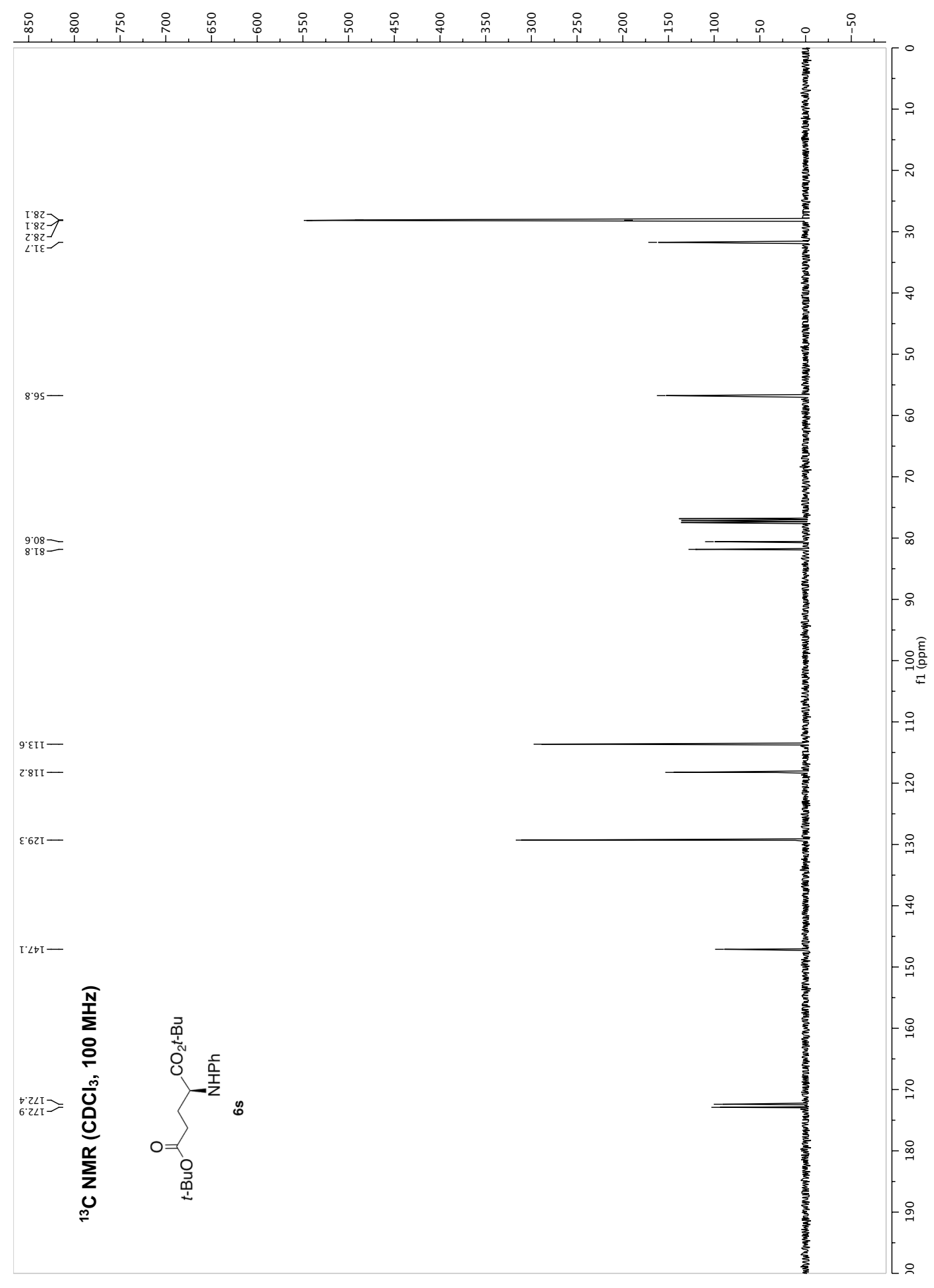




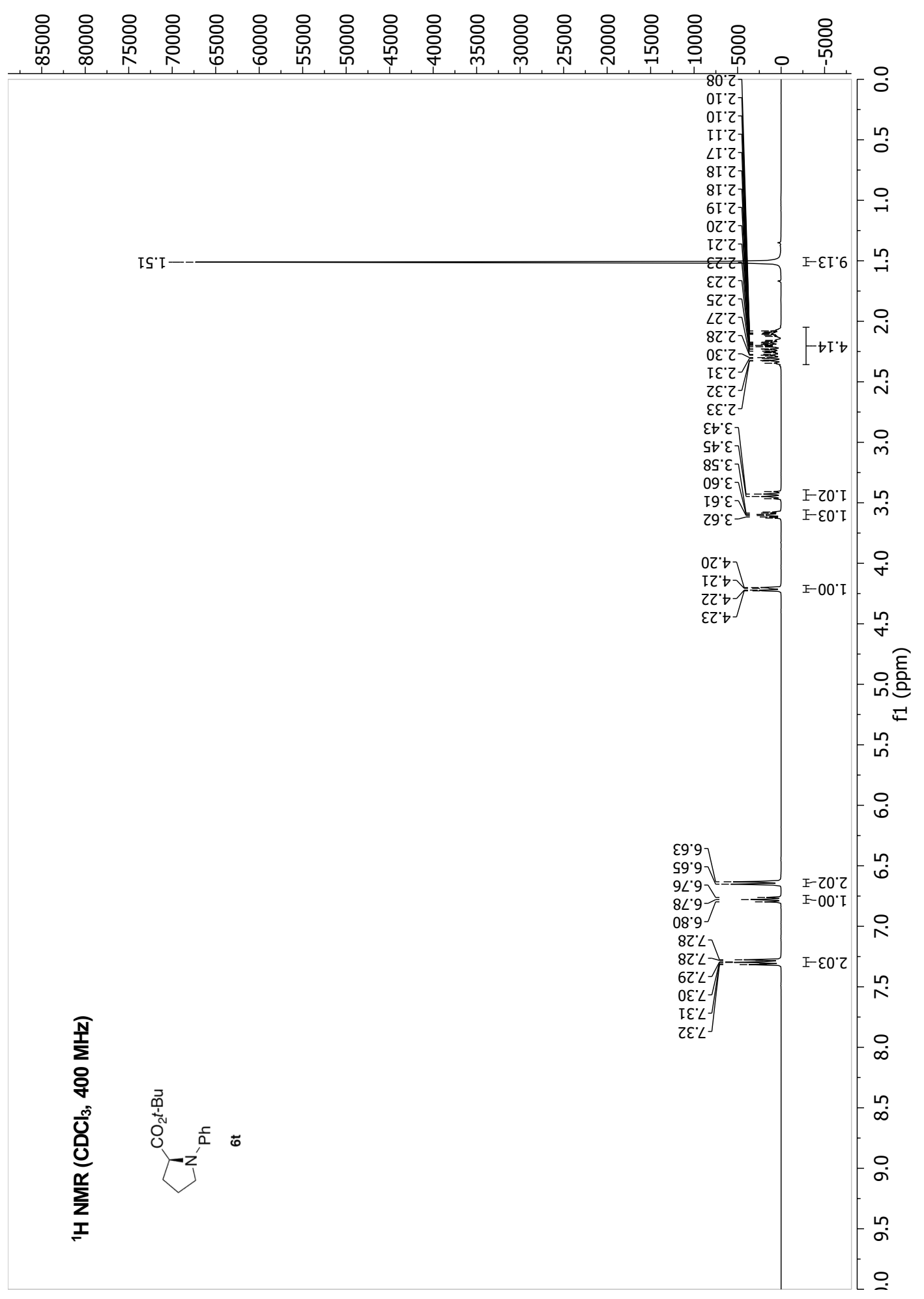




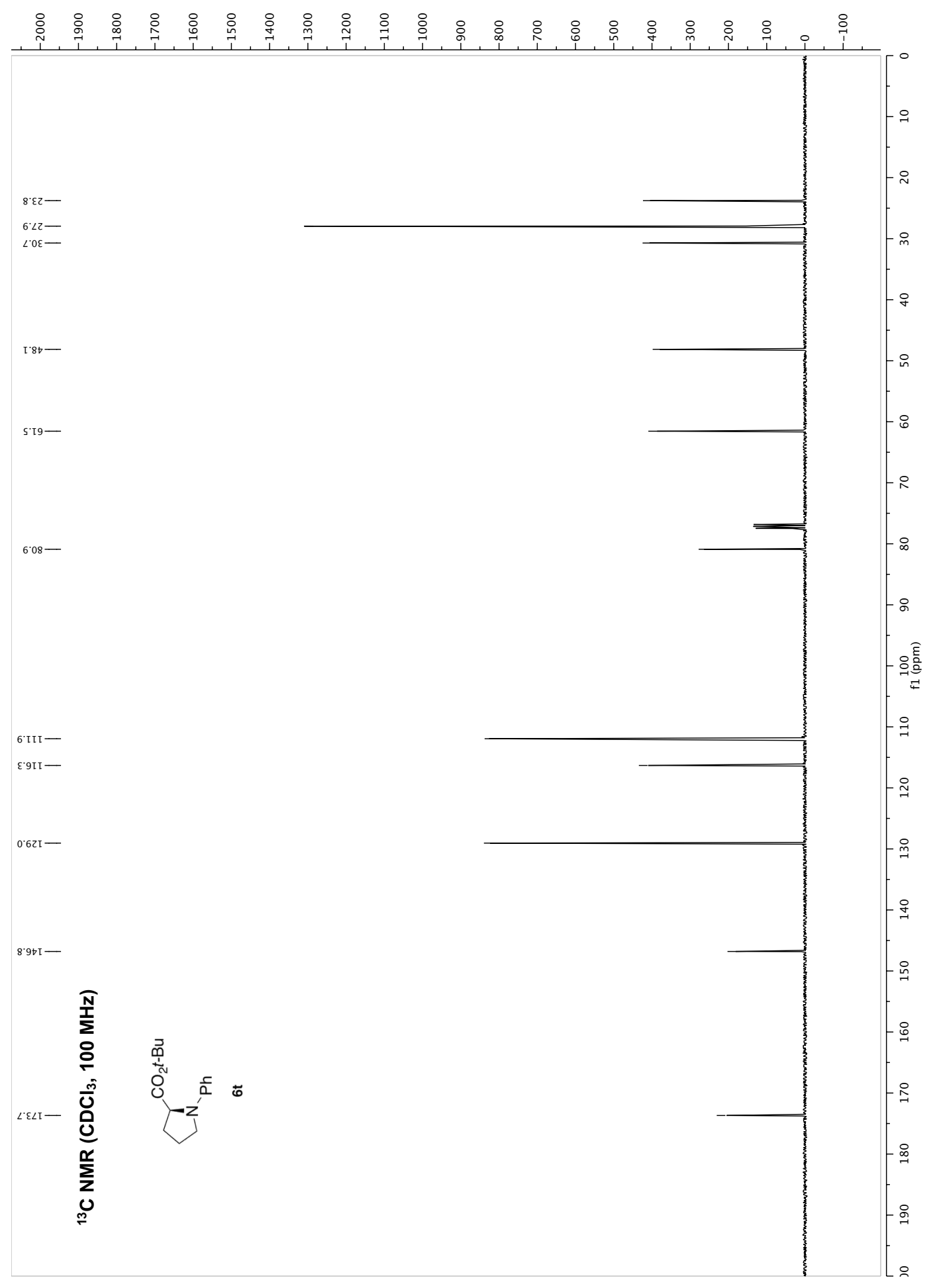




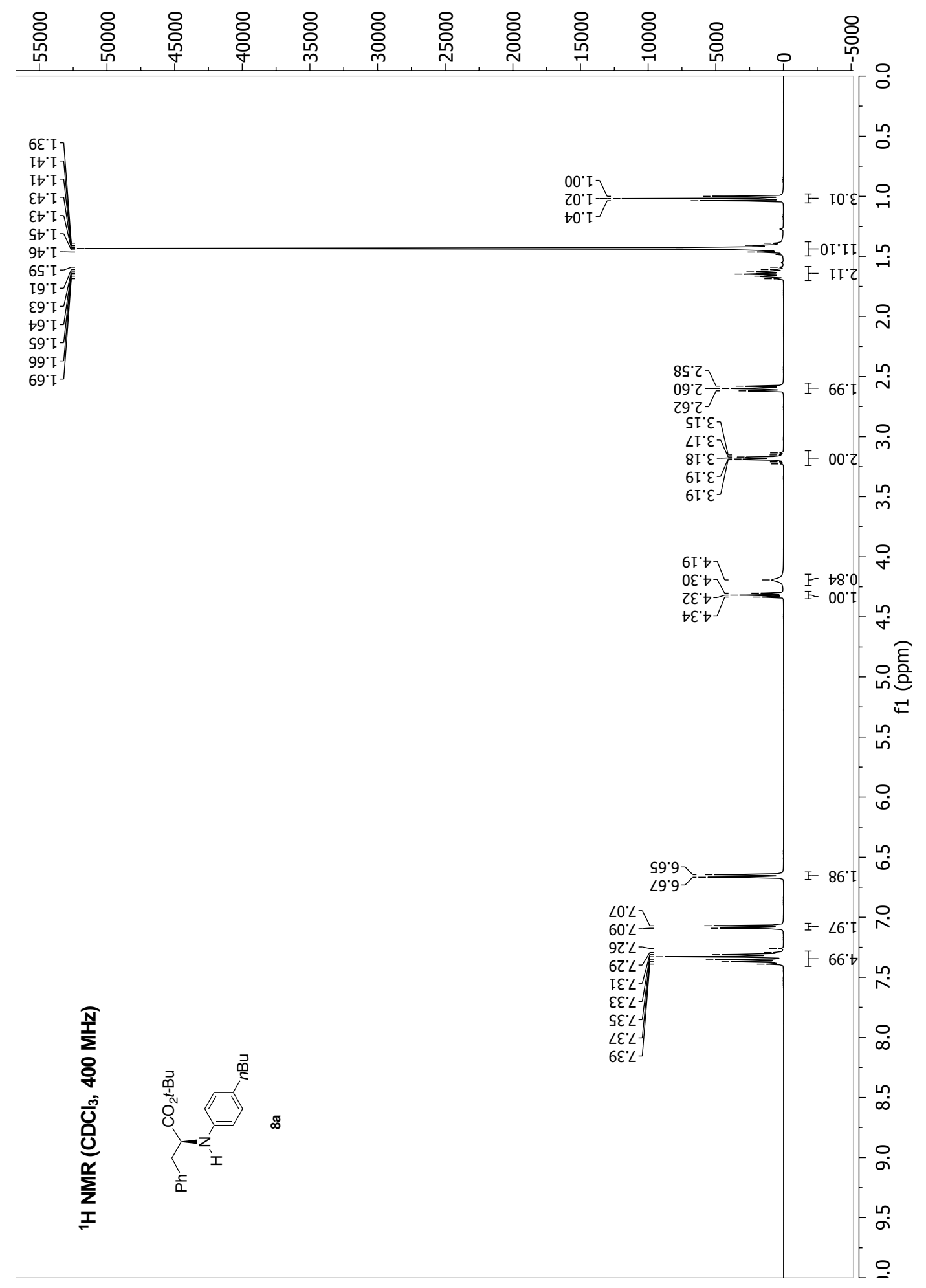




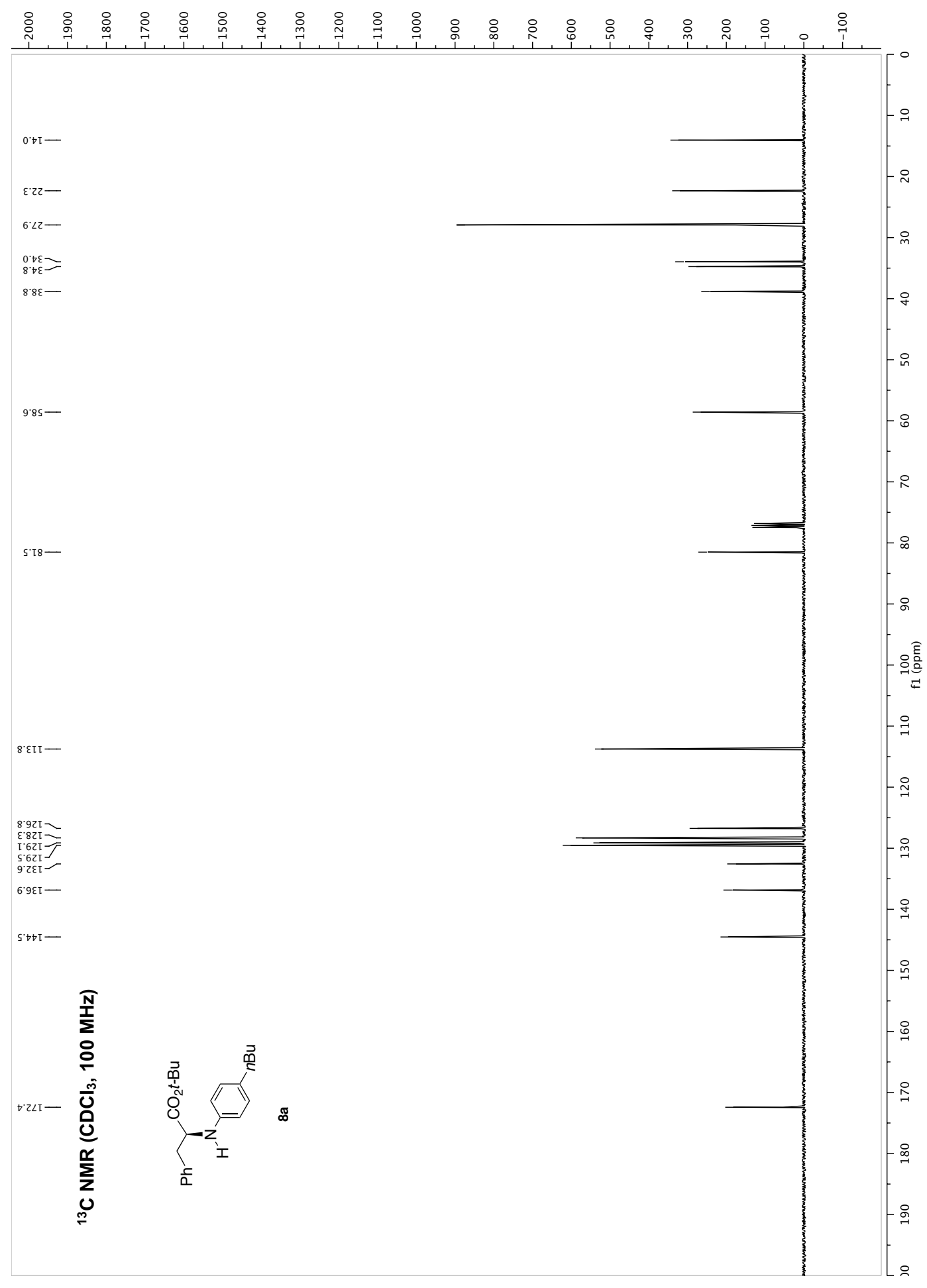




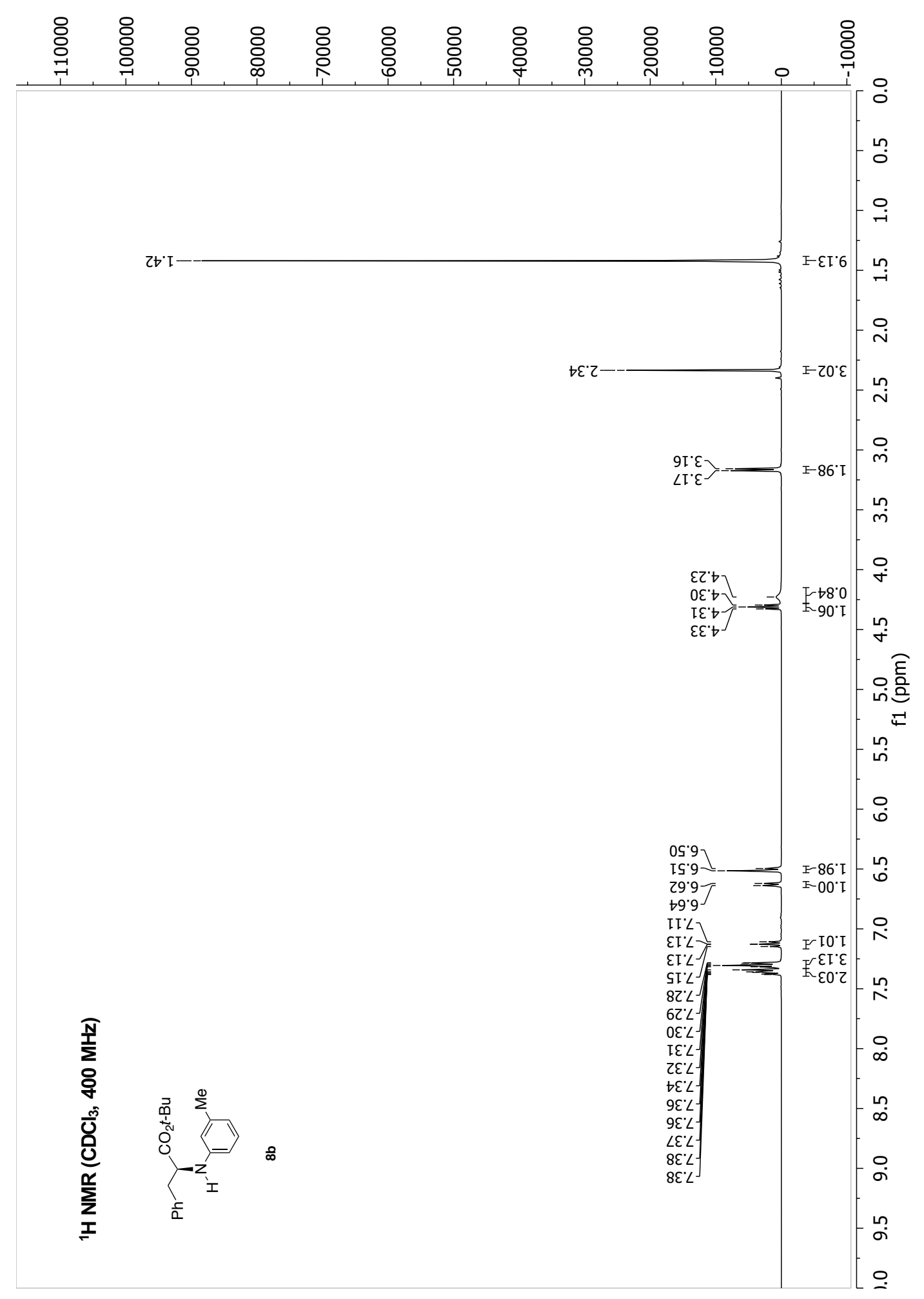




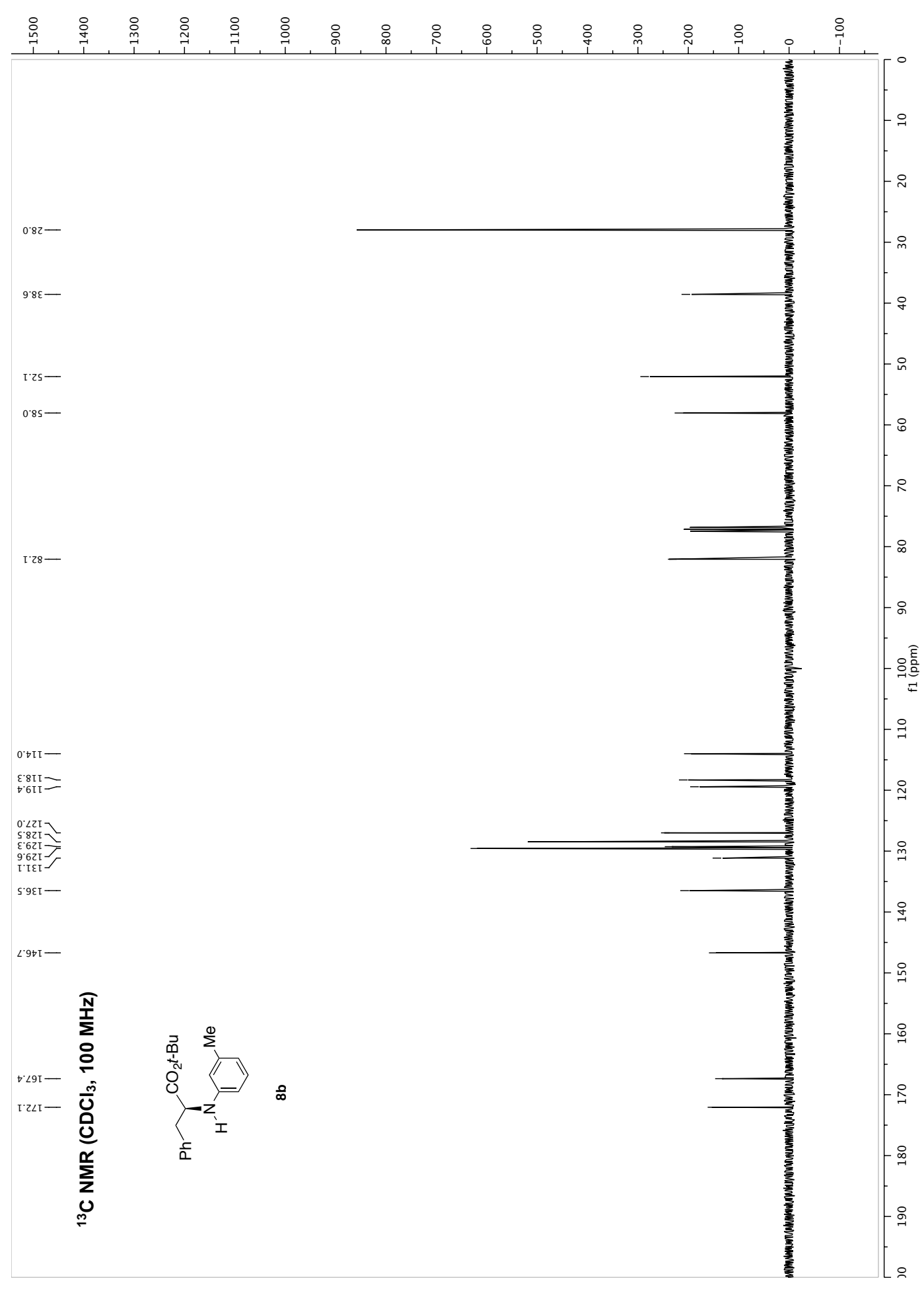




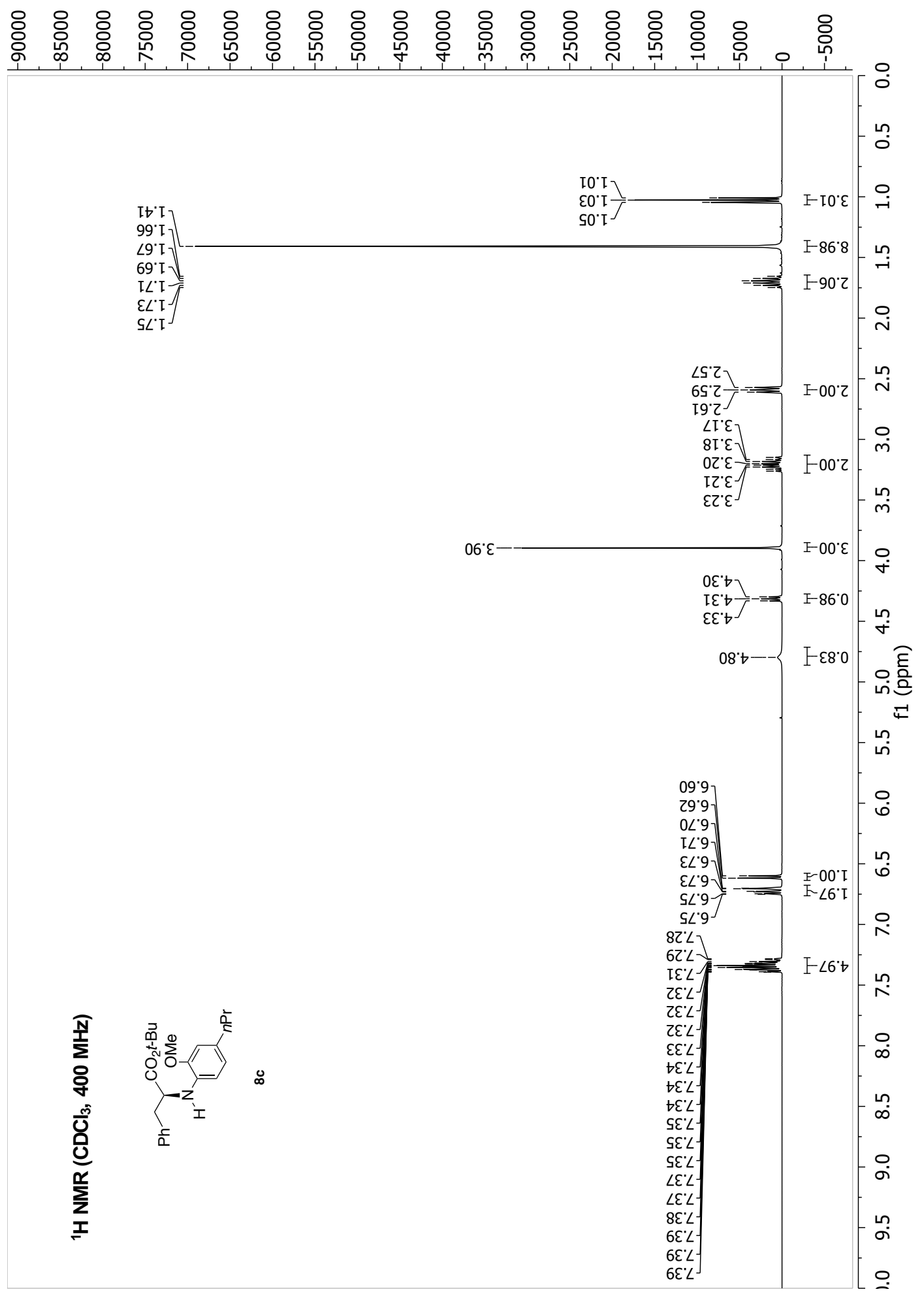




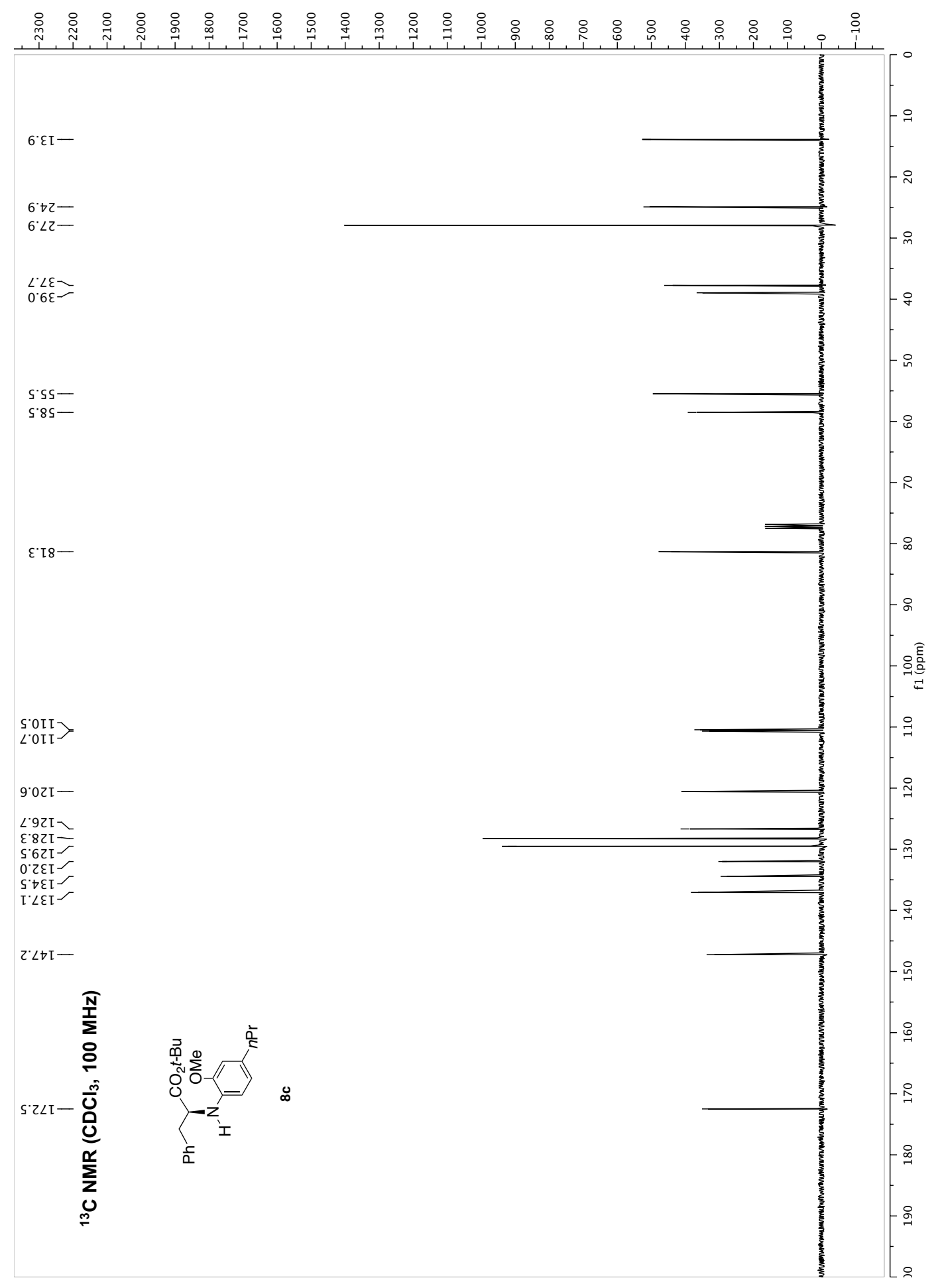




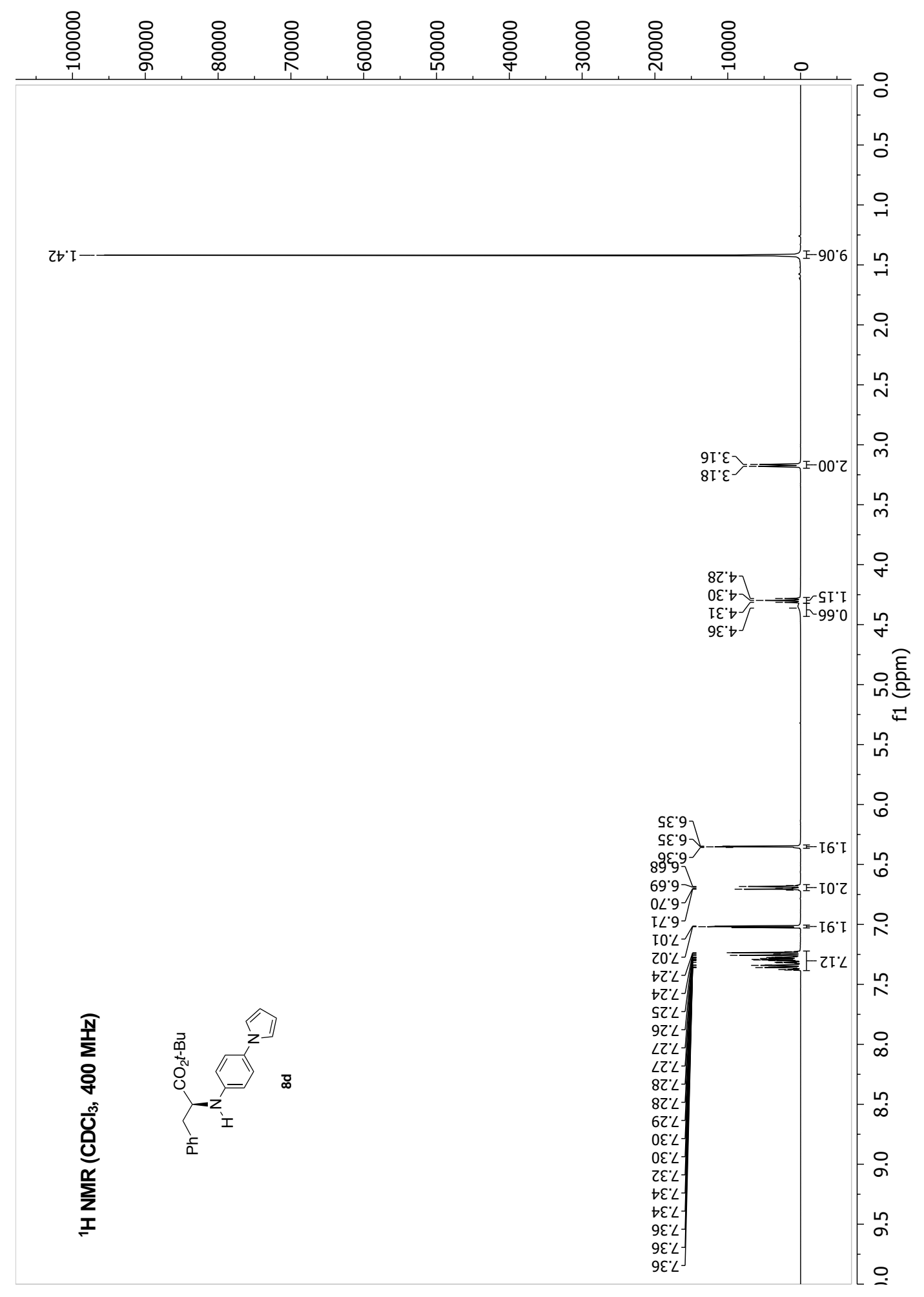




$$
7
$$




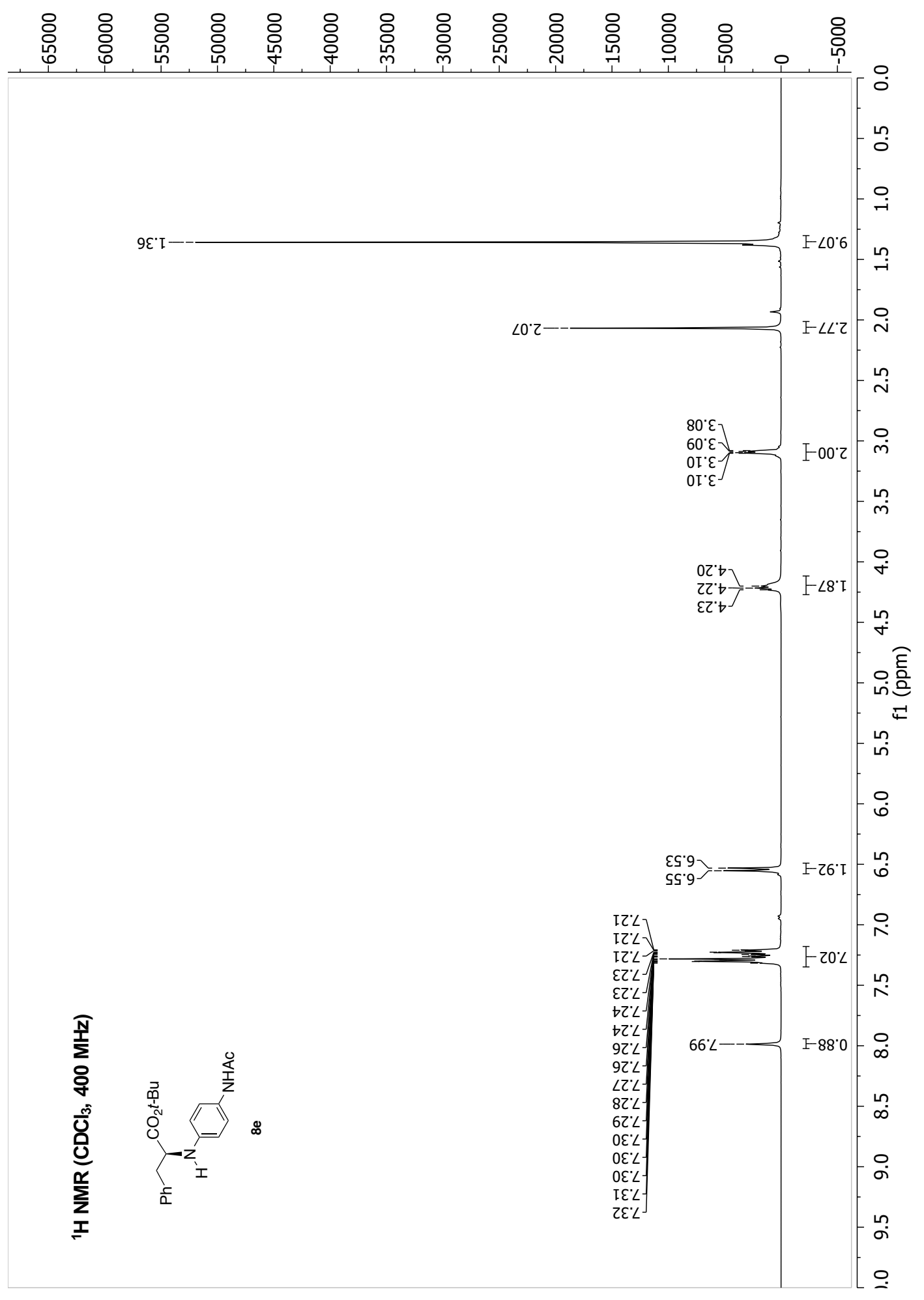




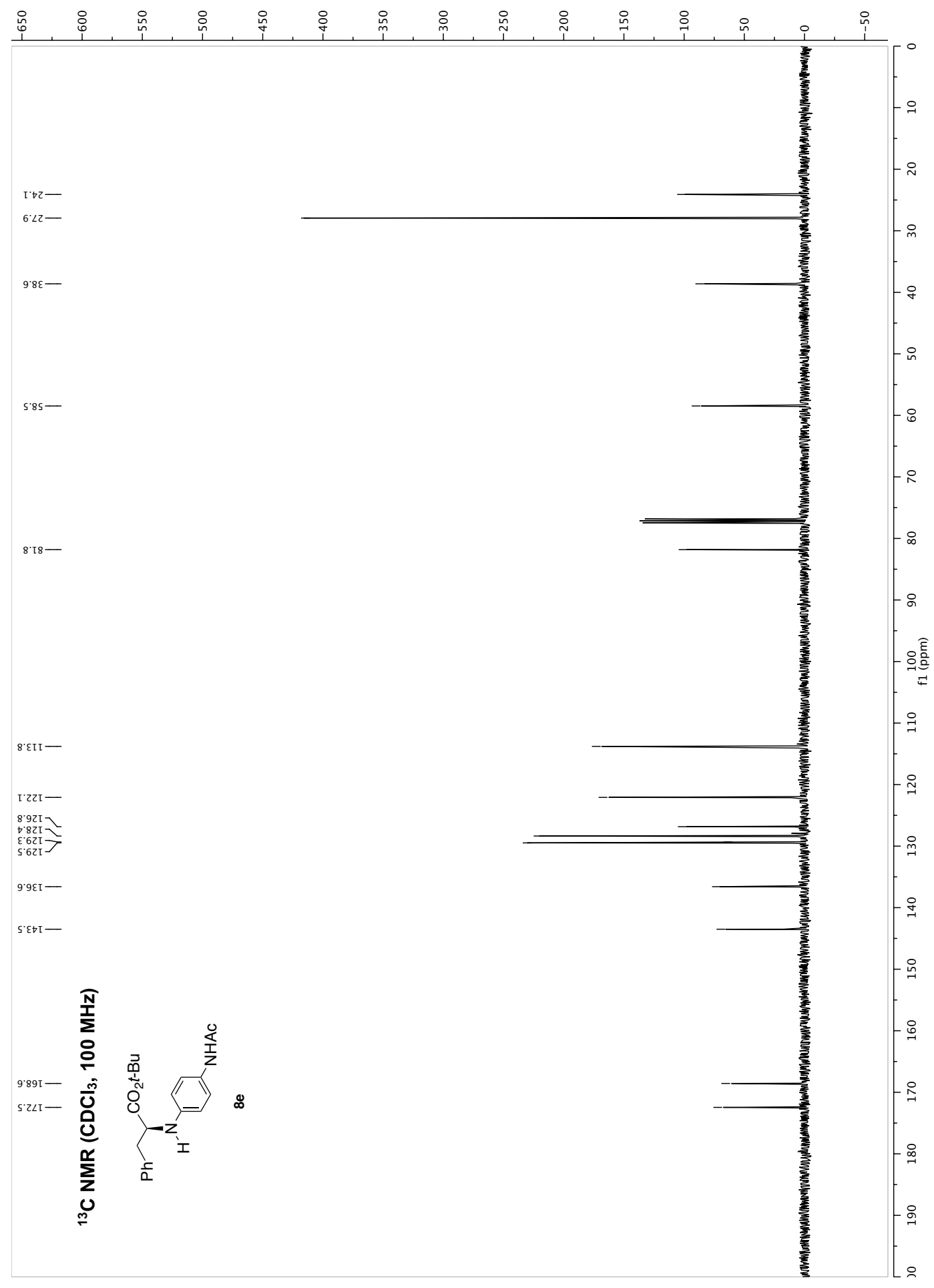




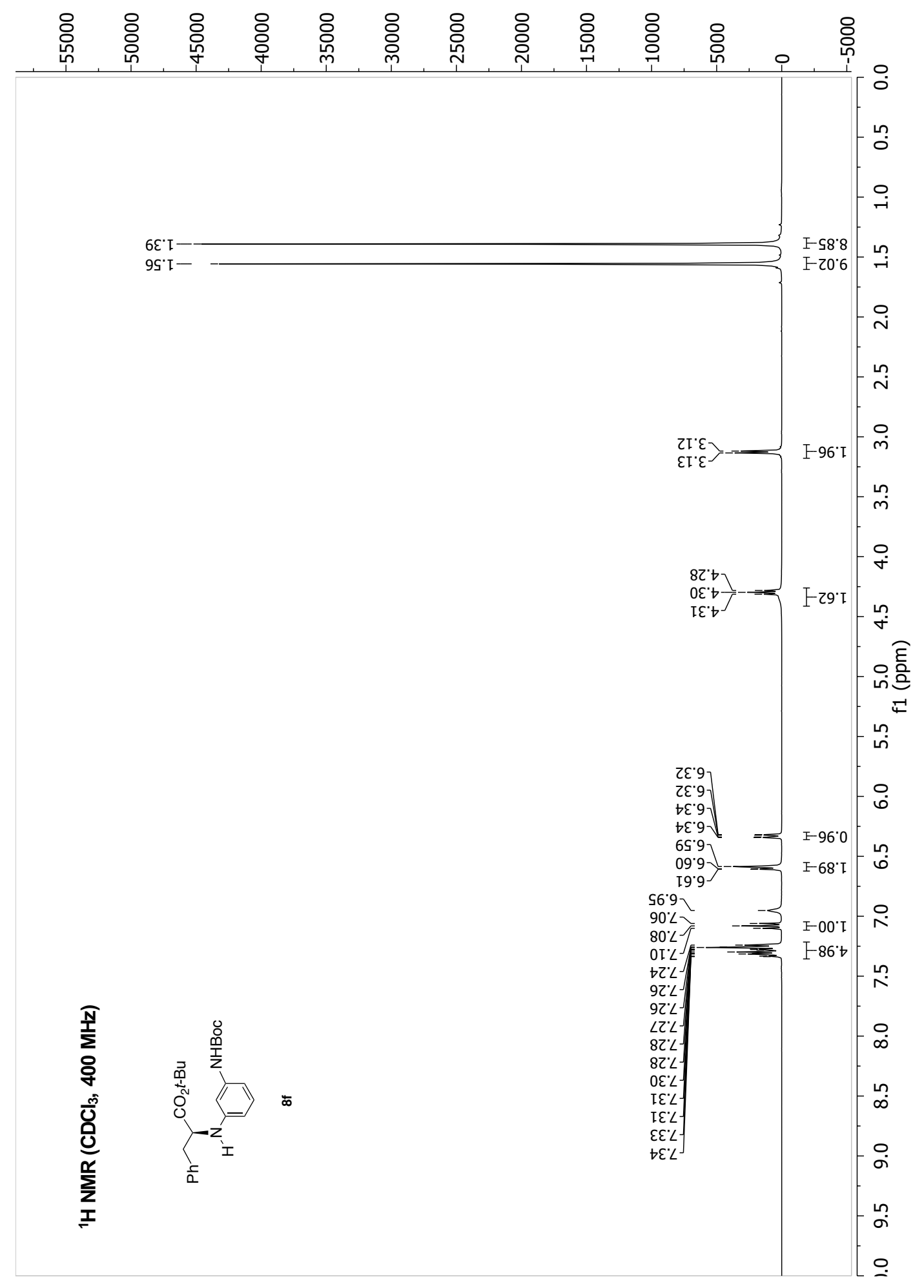




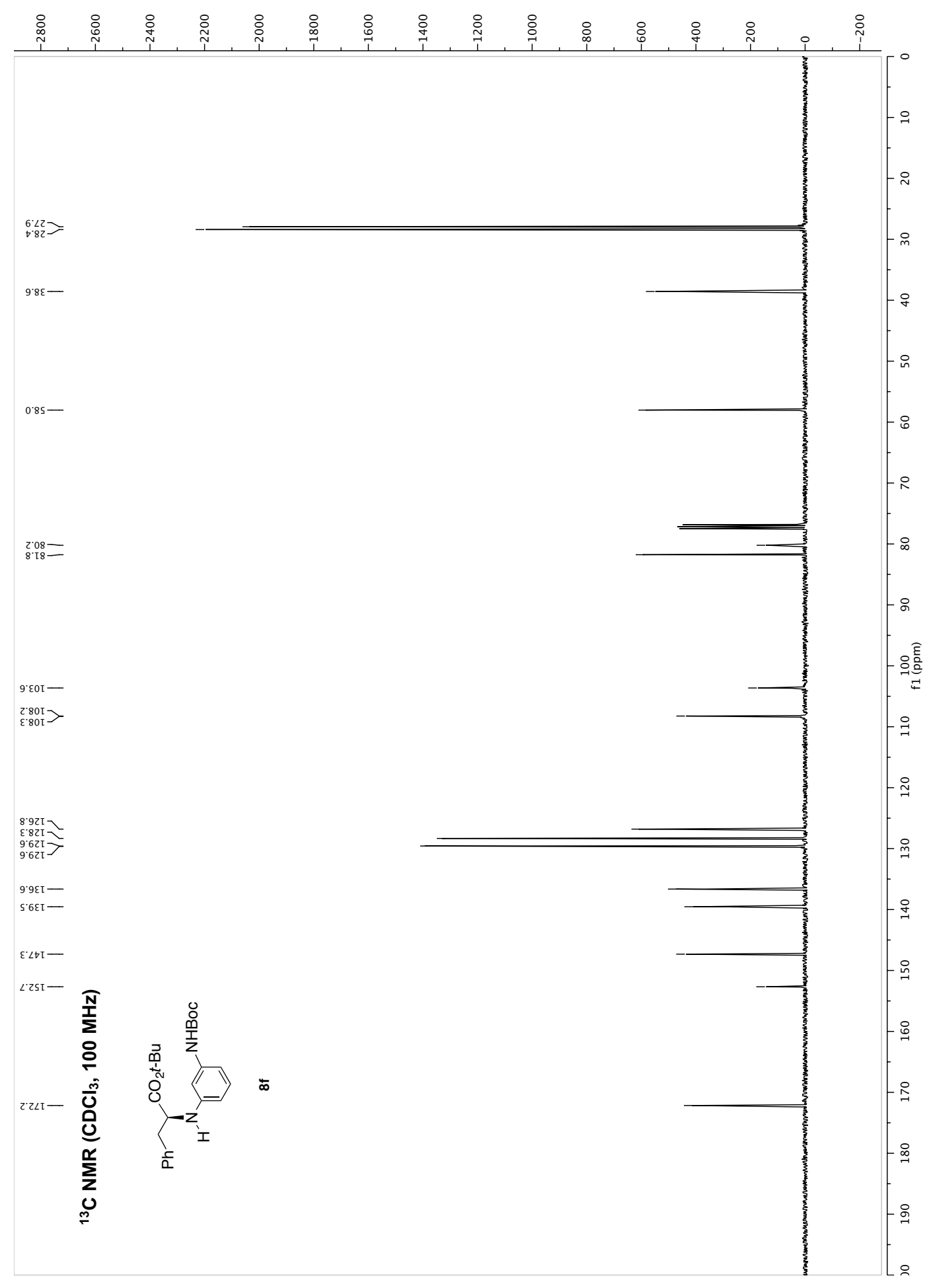




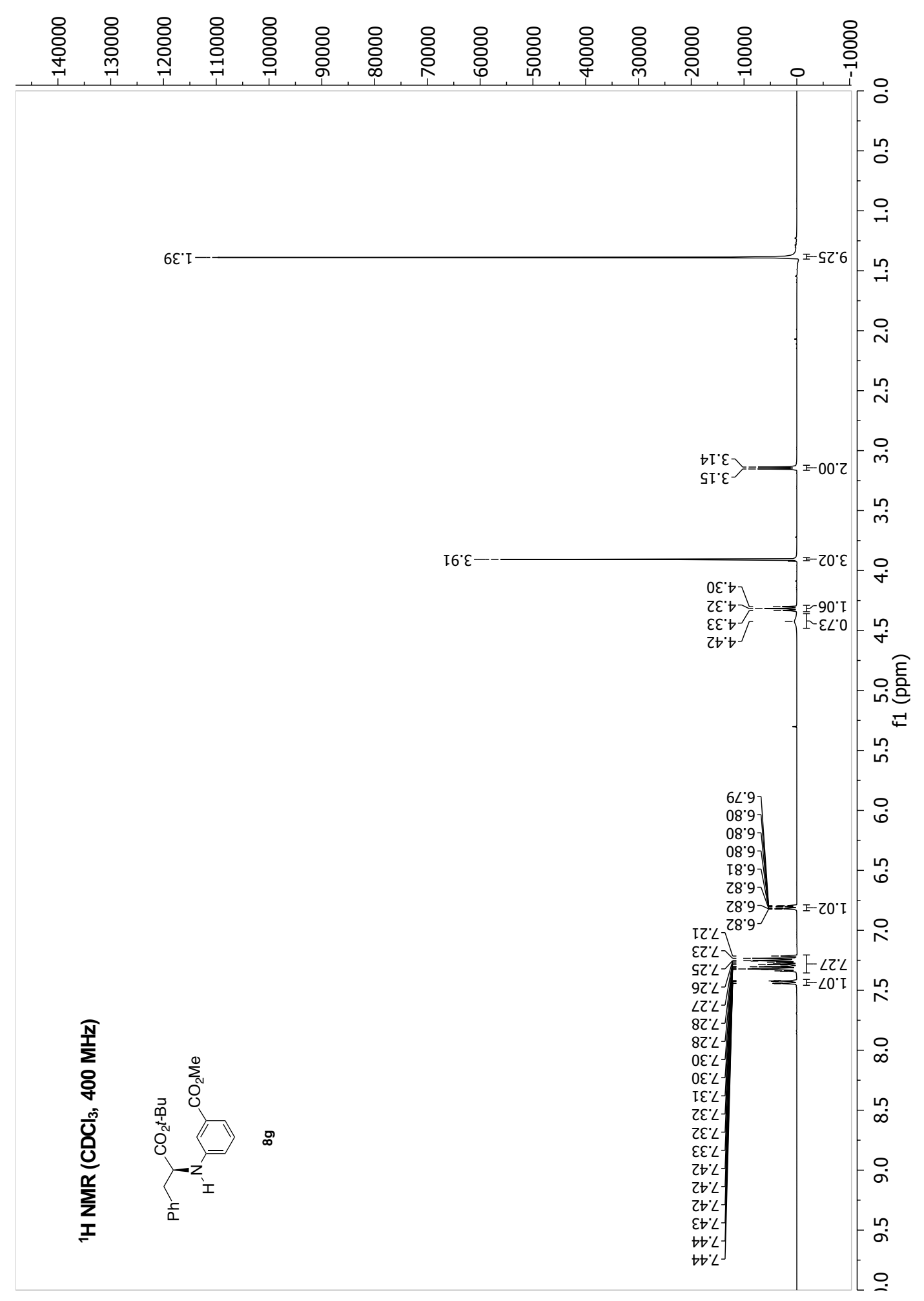




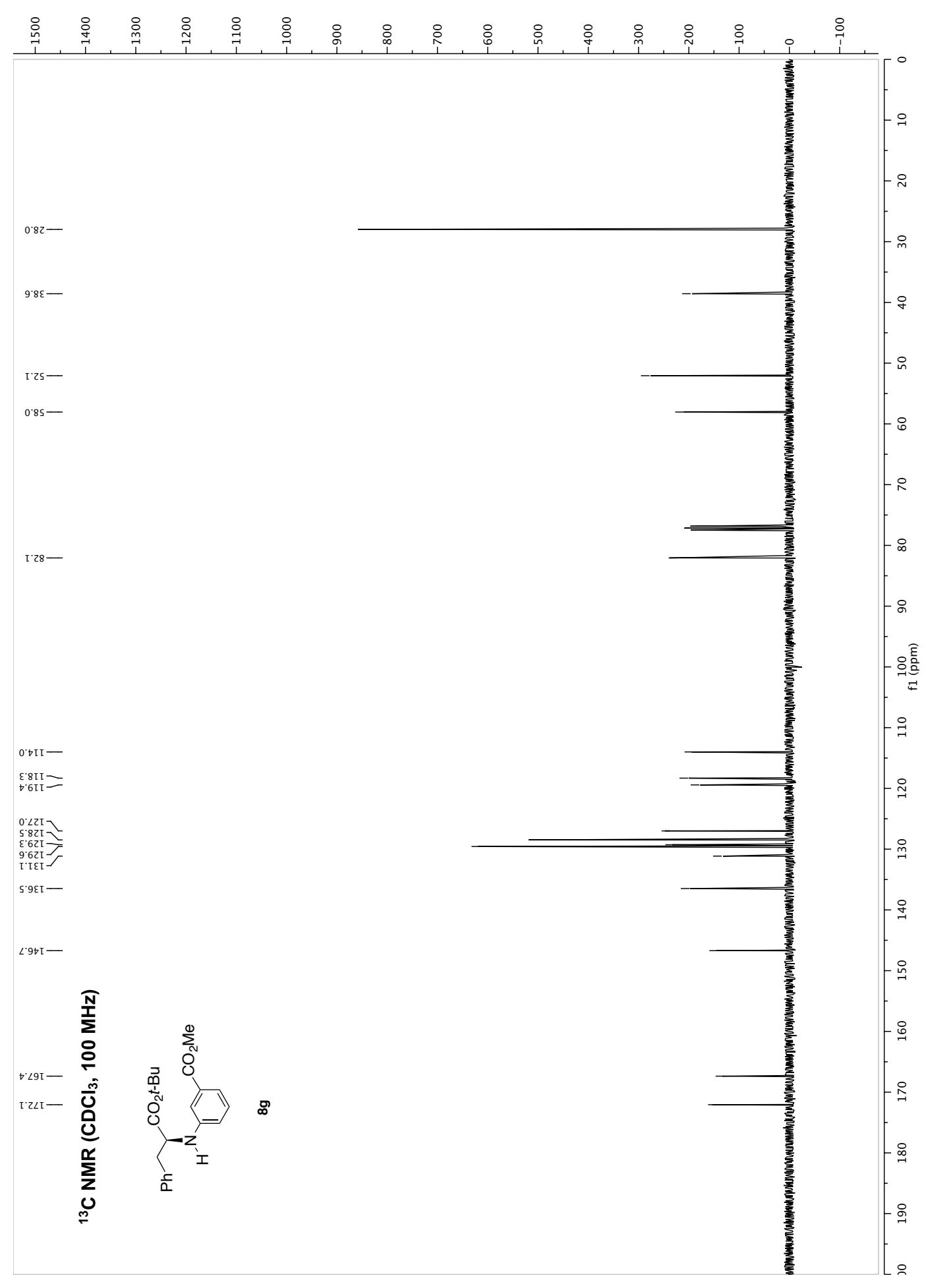




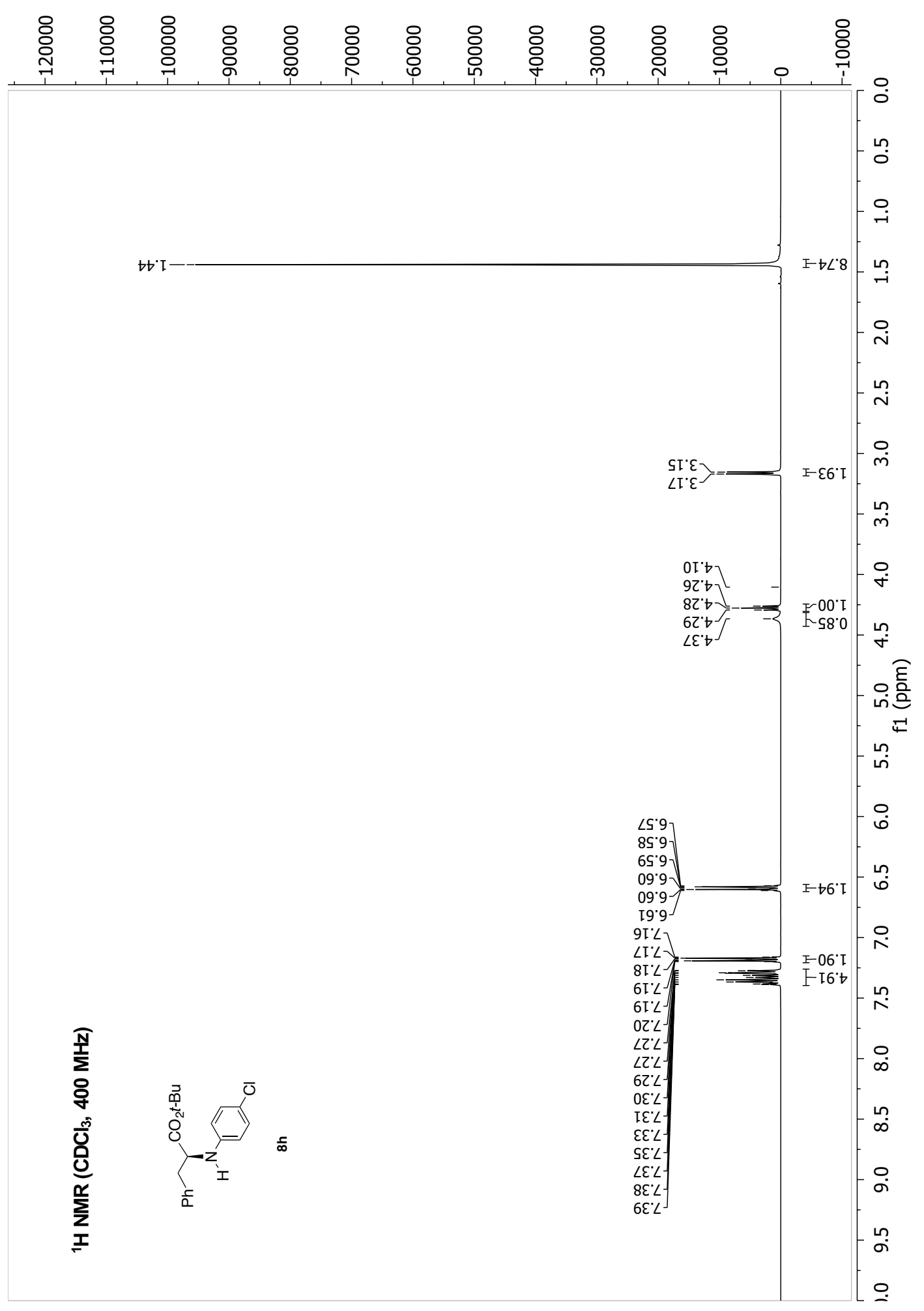




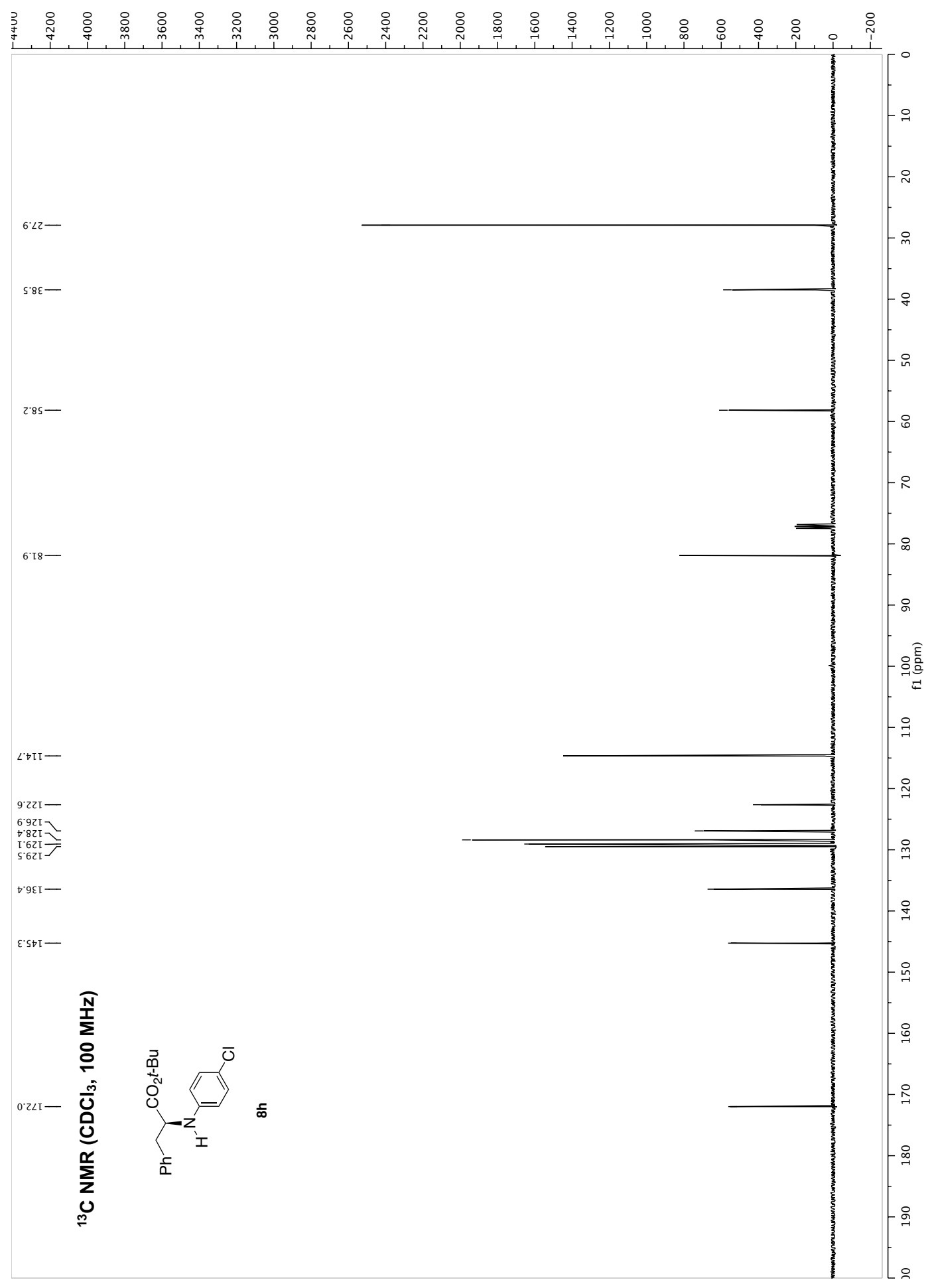




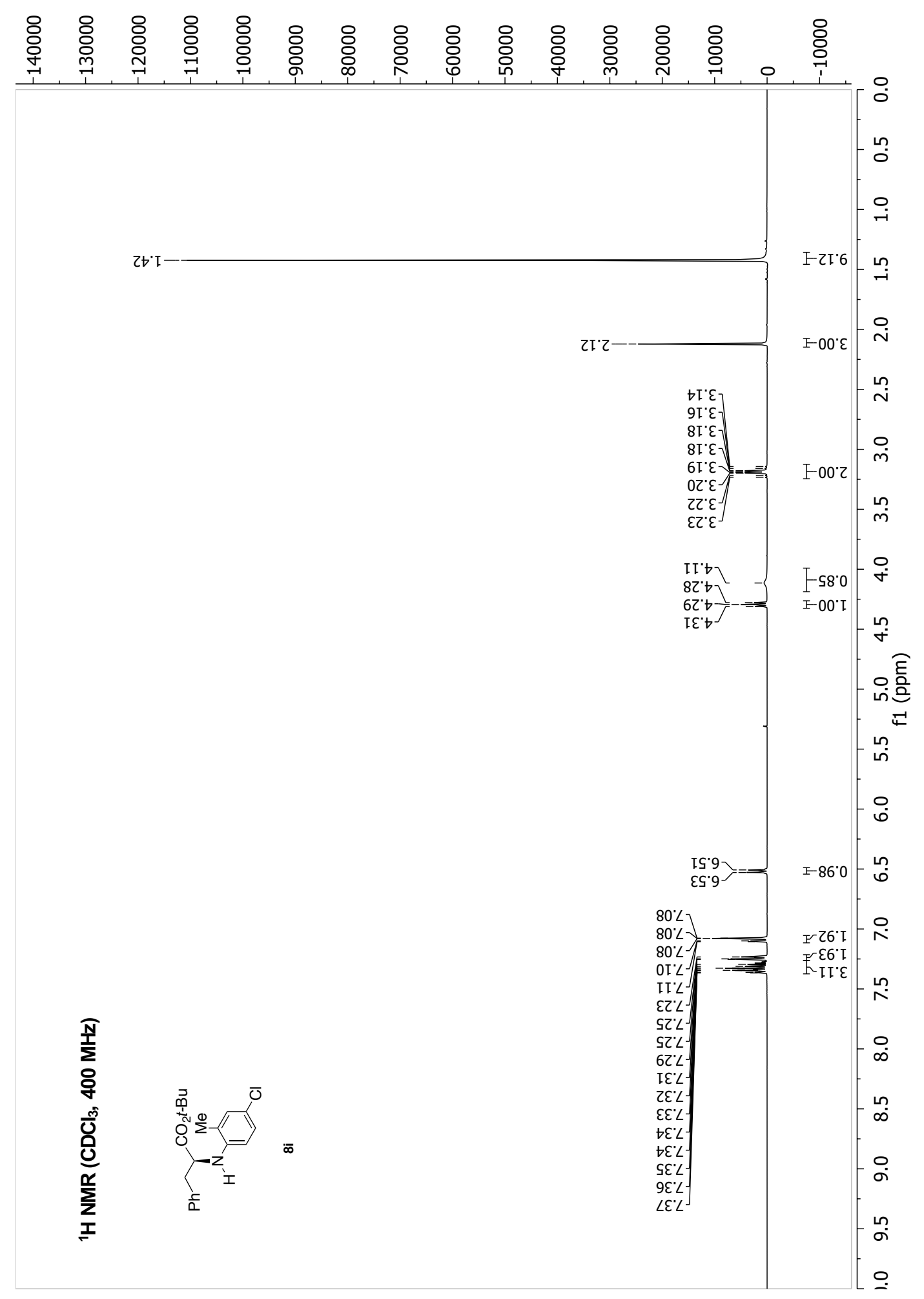




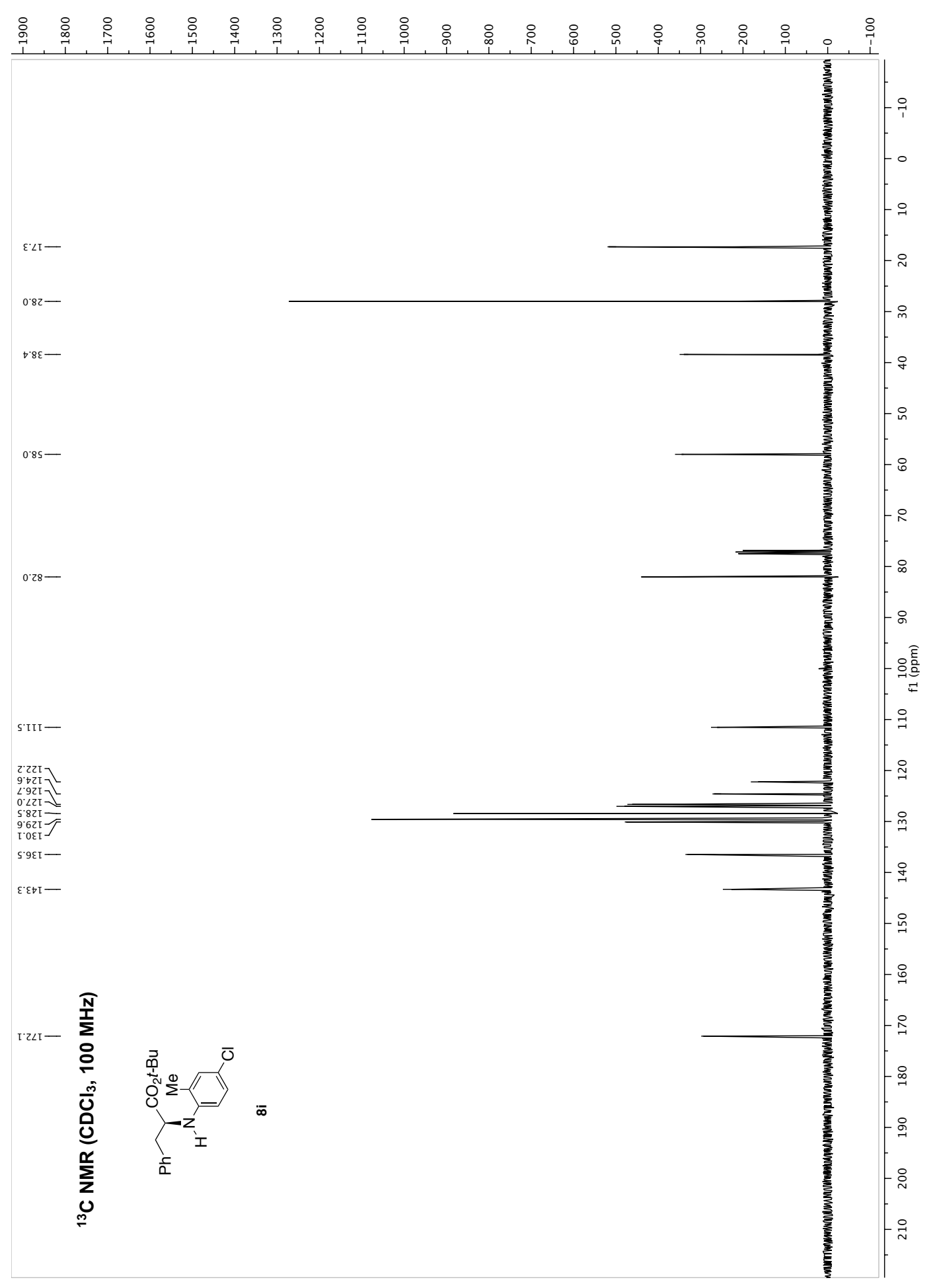




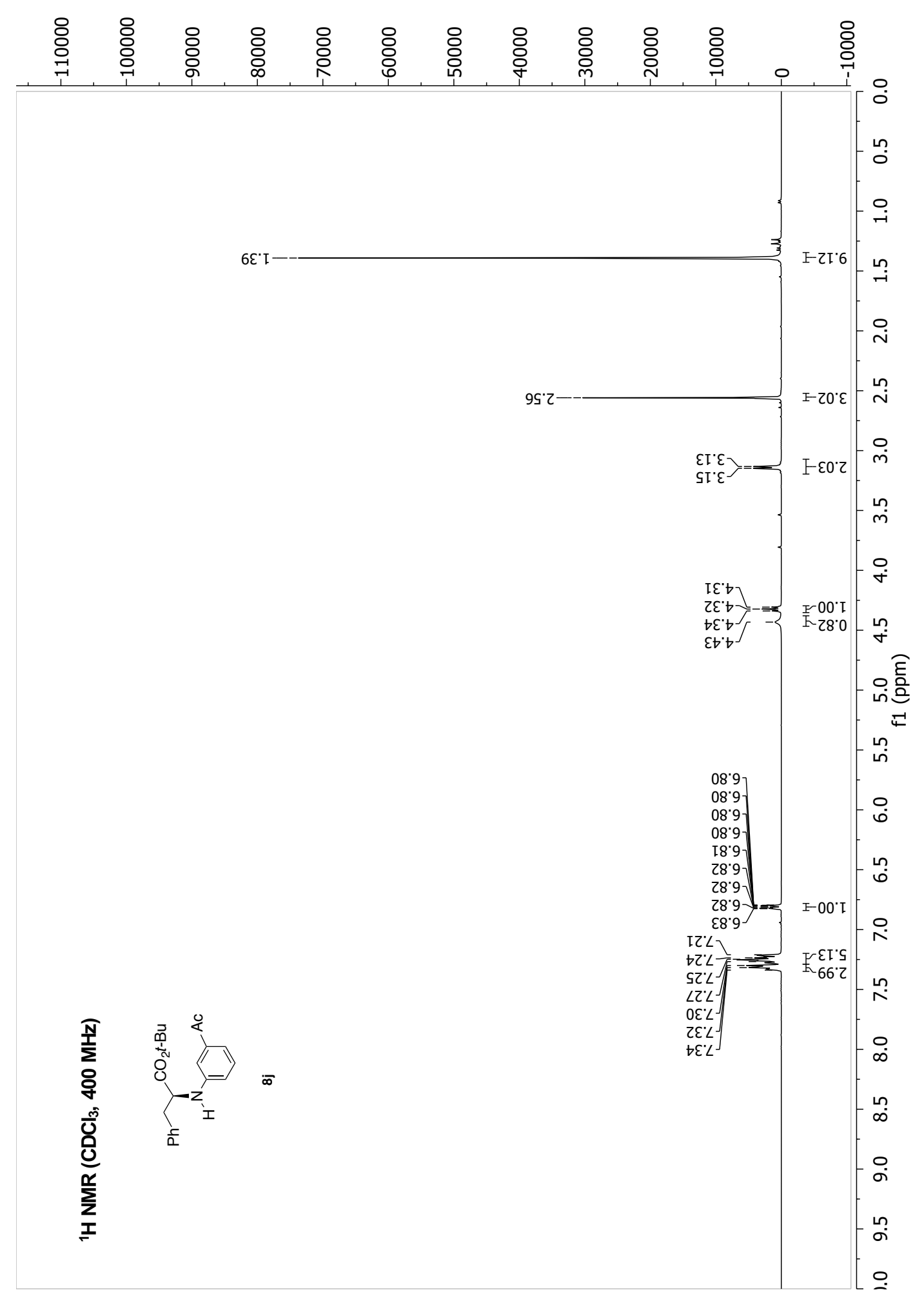




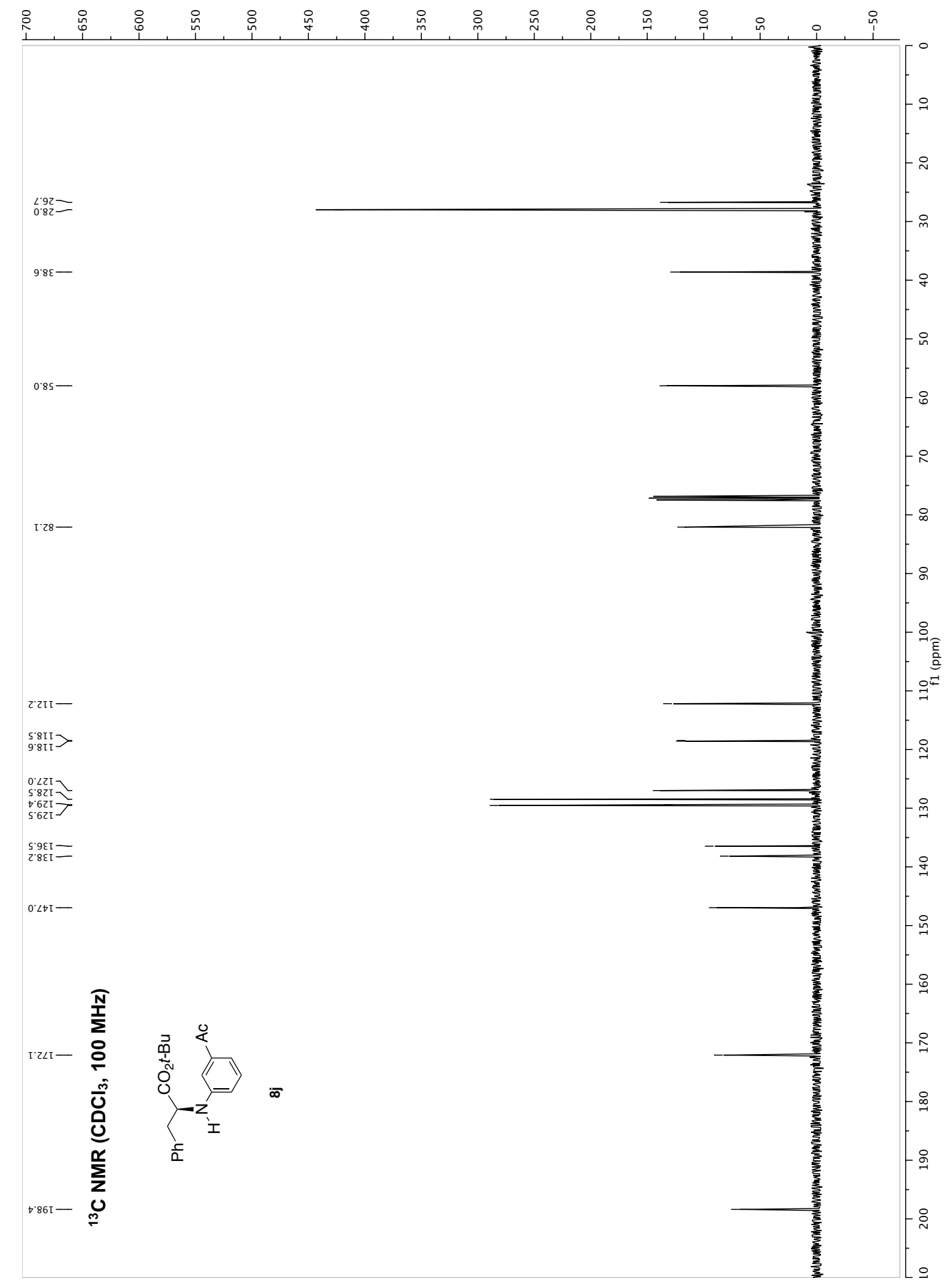




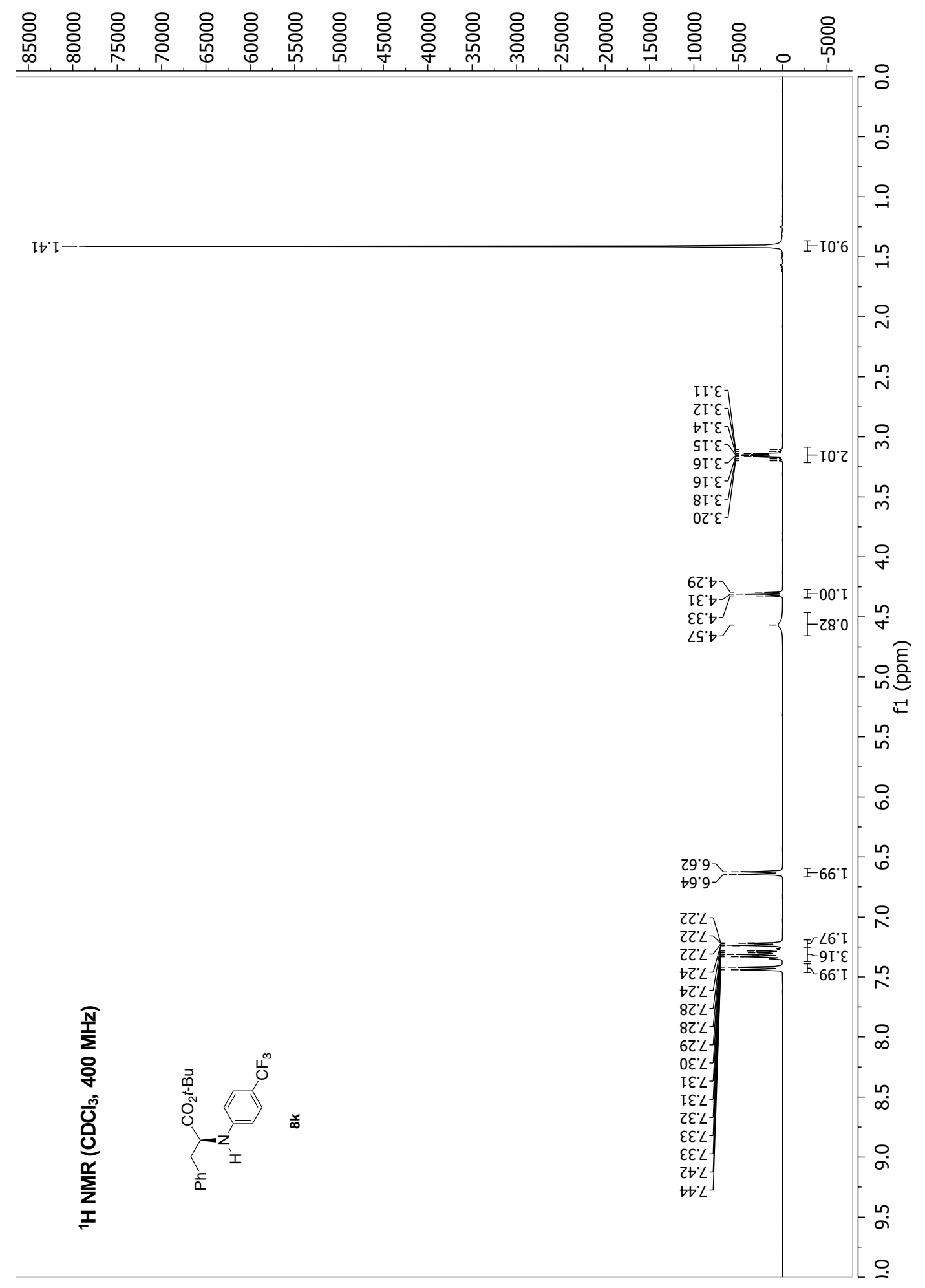




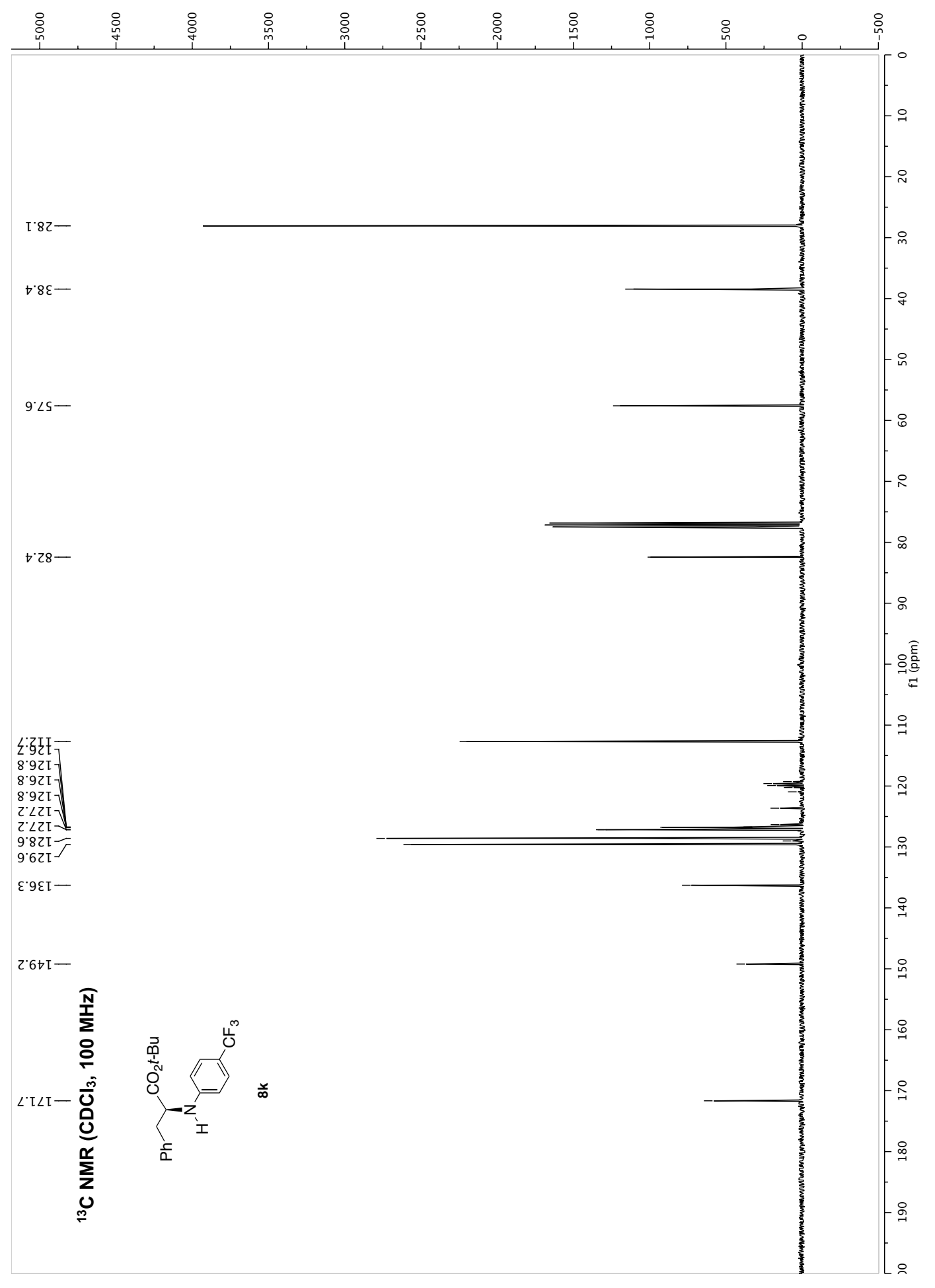




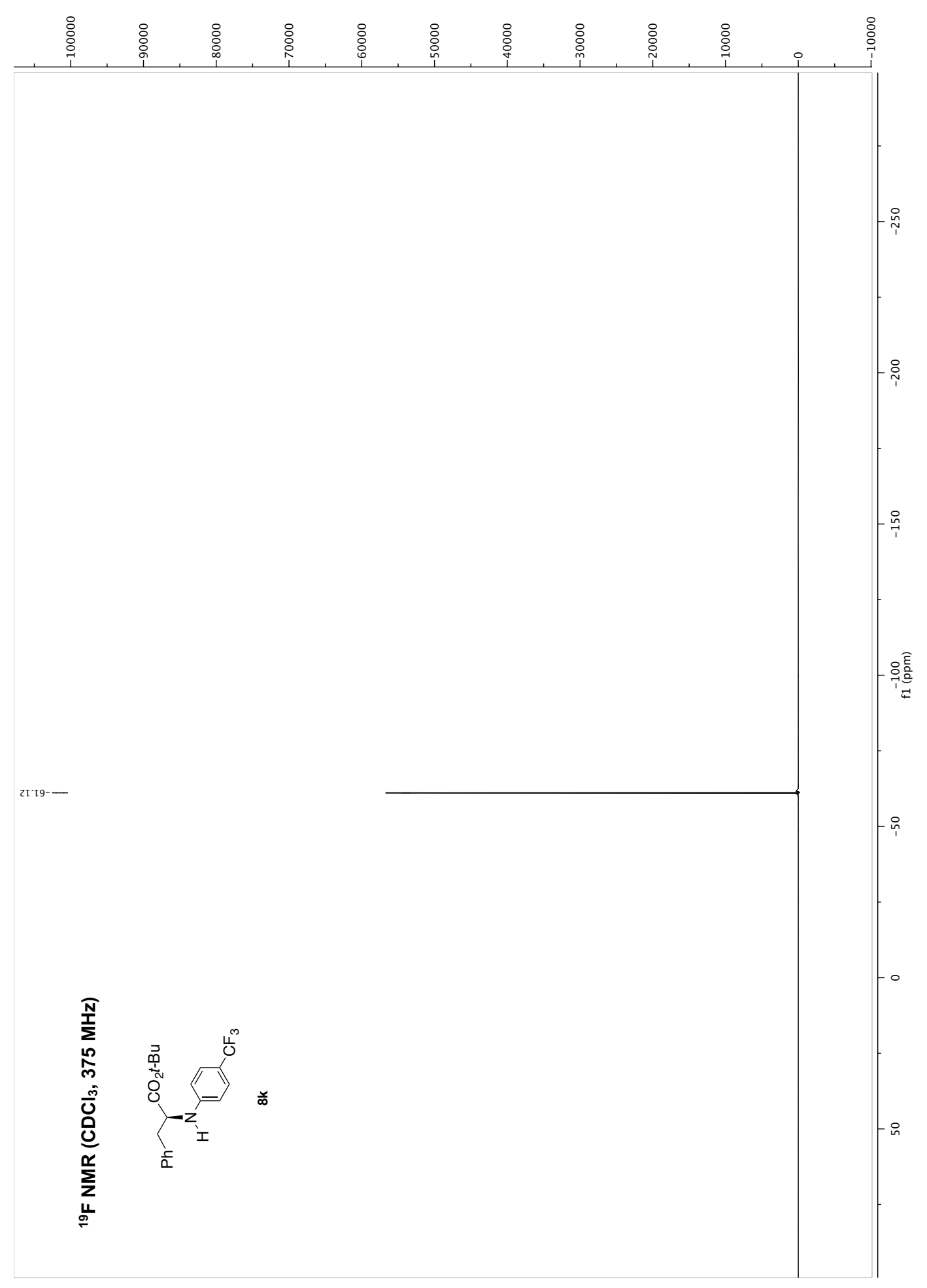




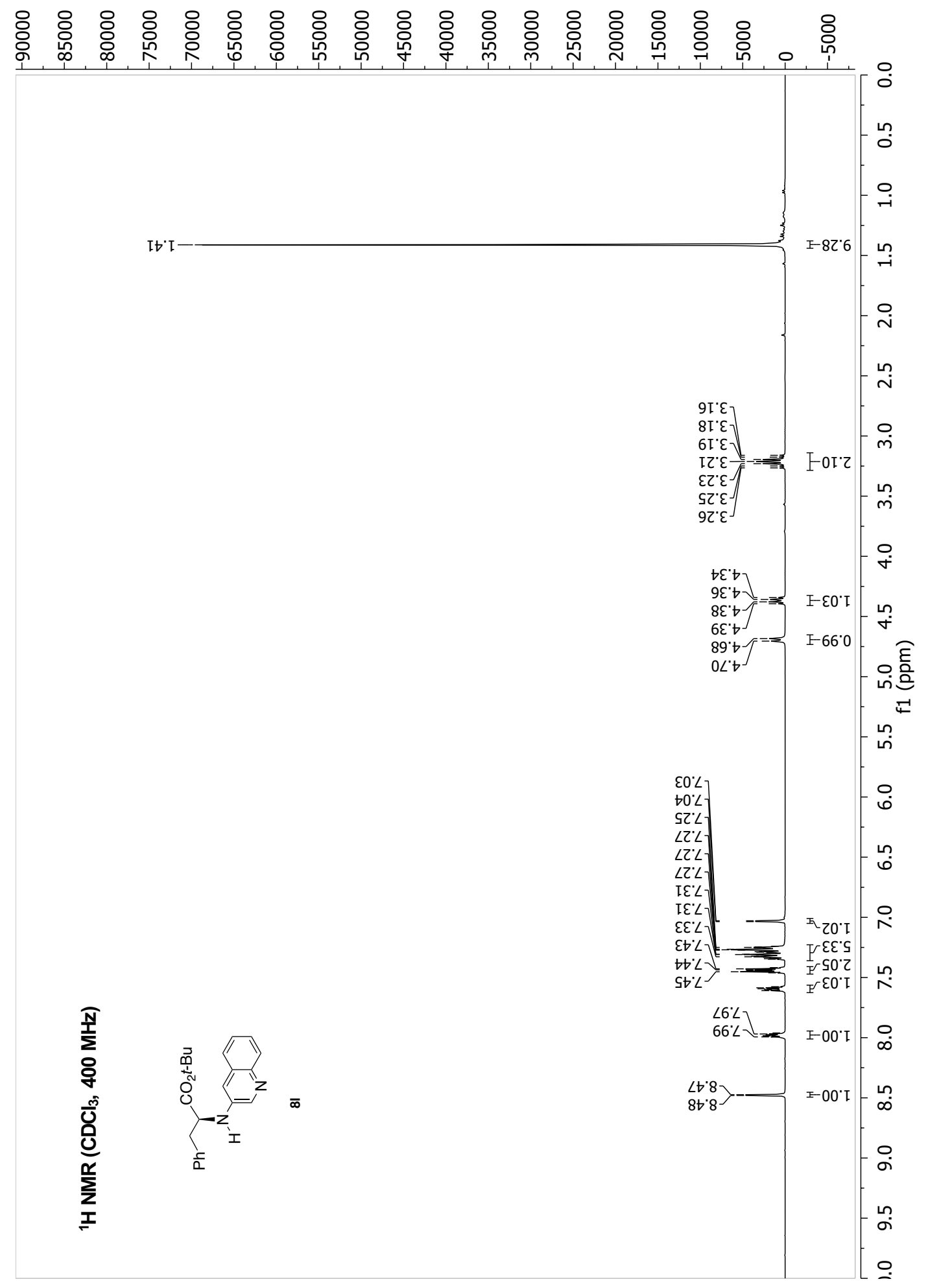




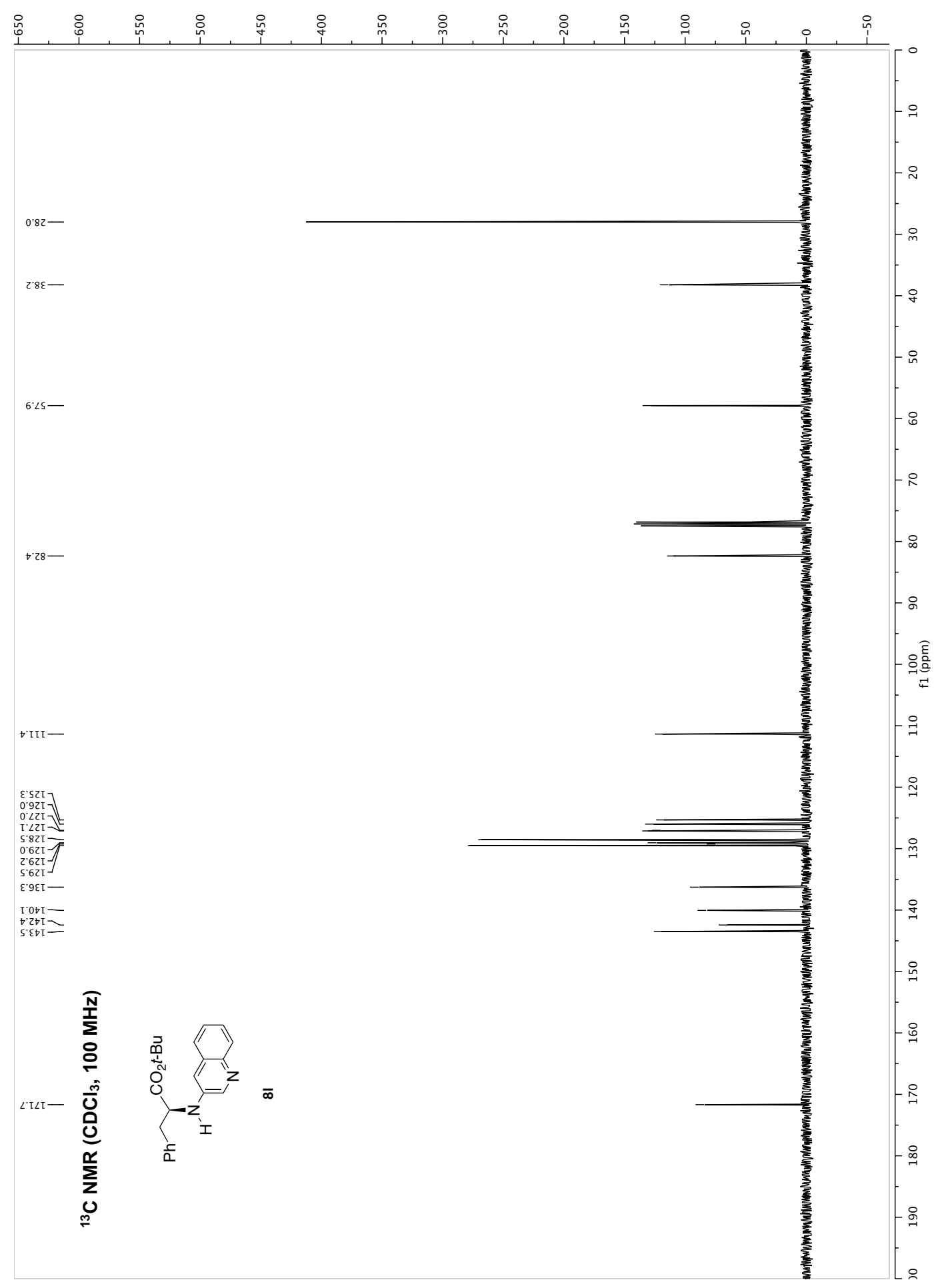




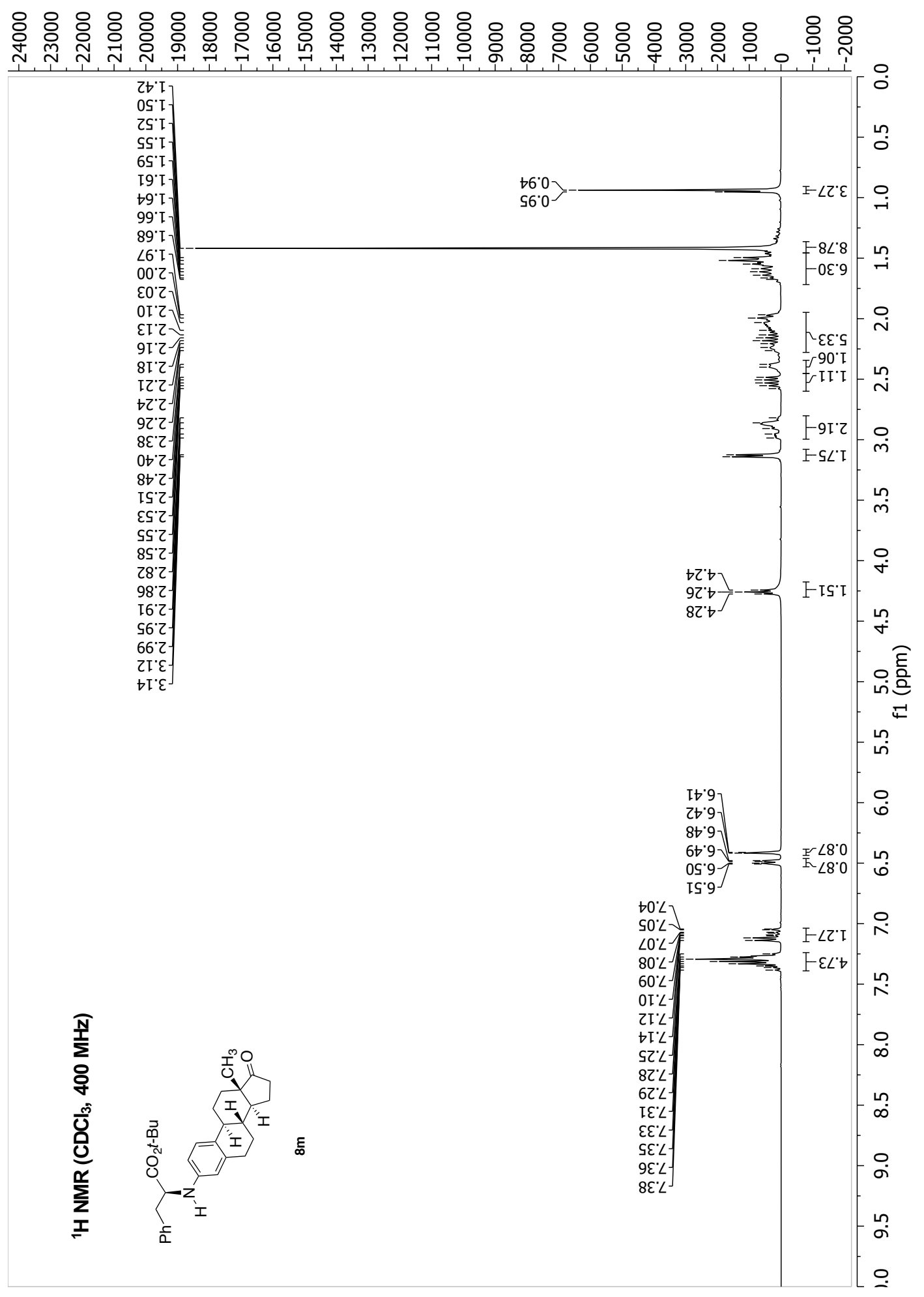




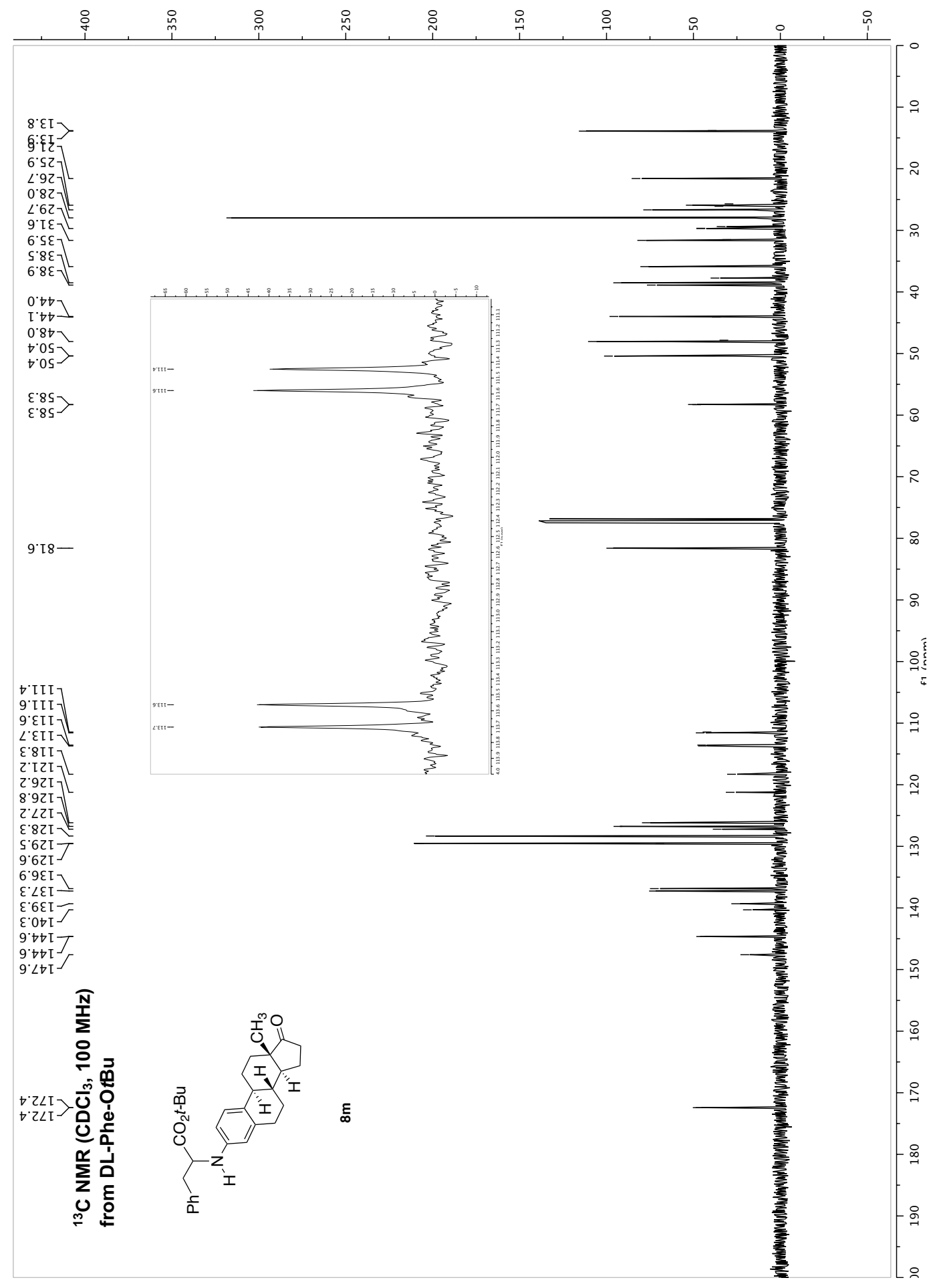




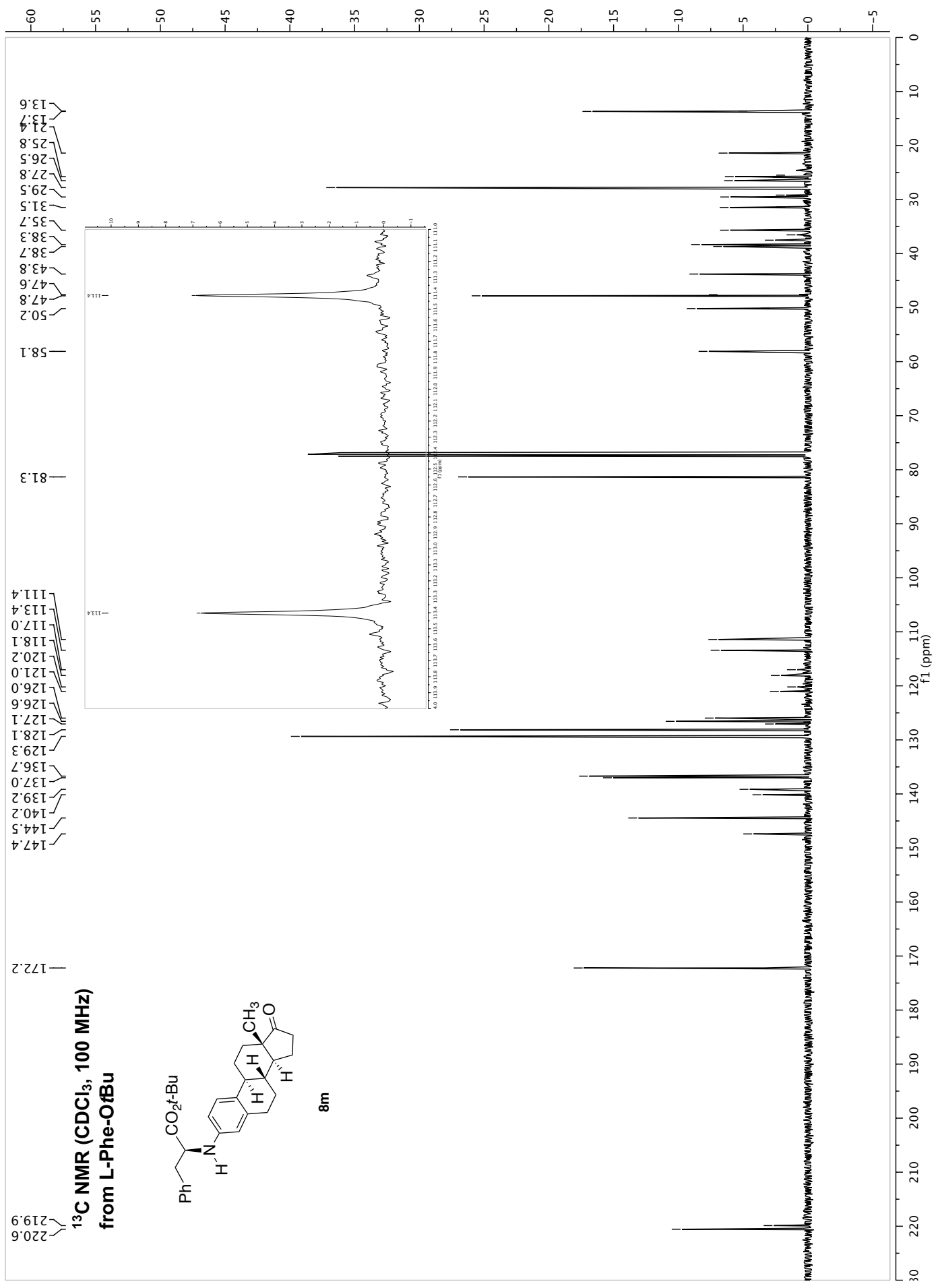




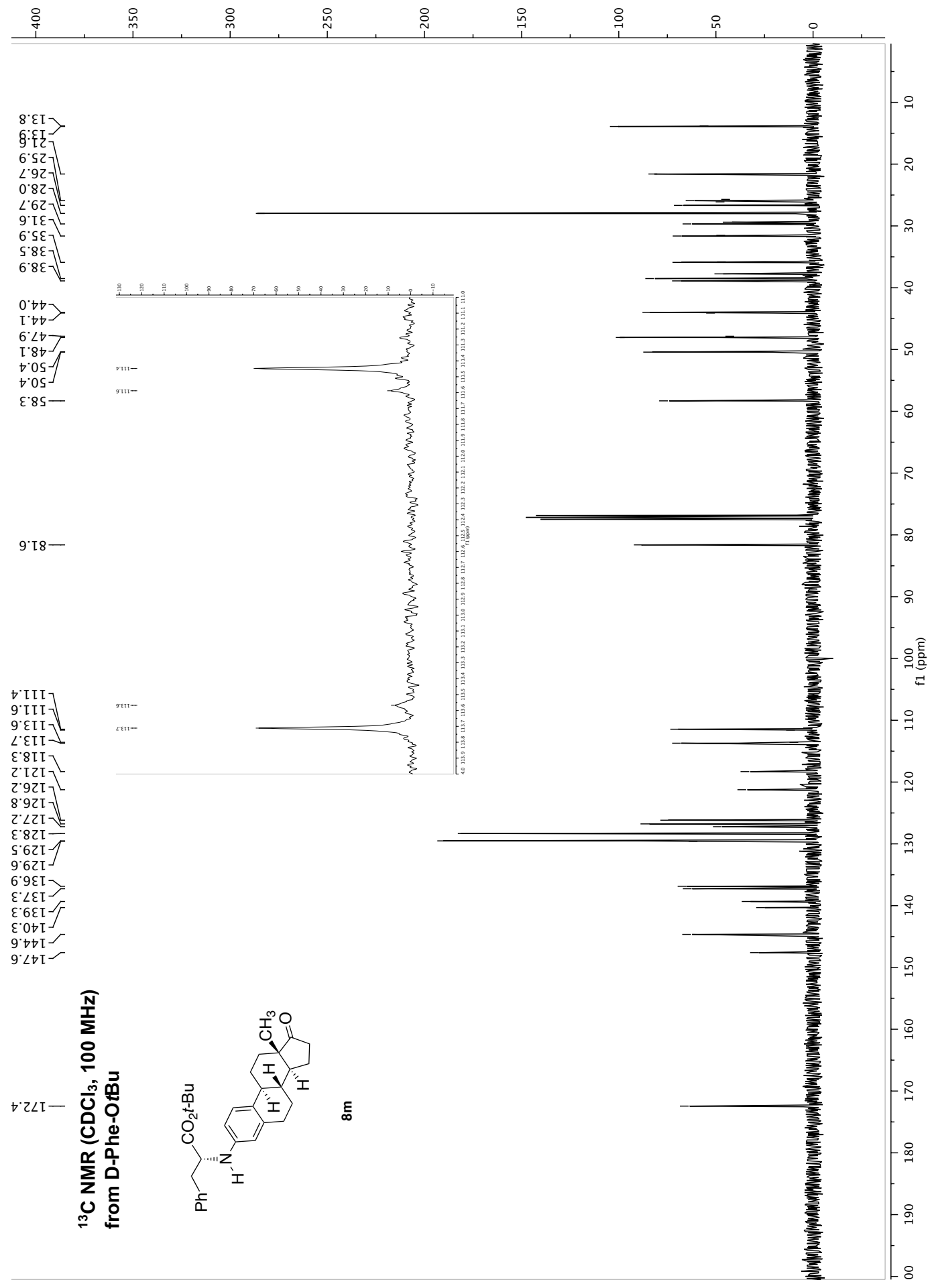




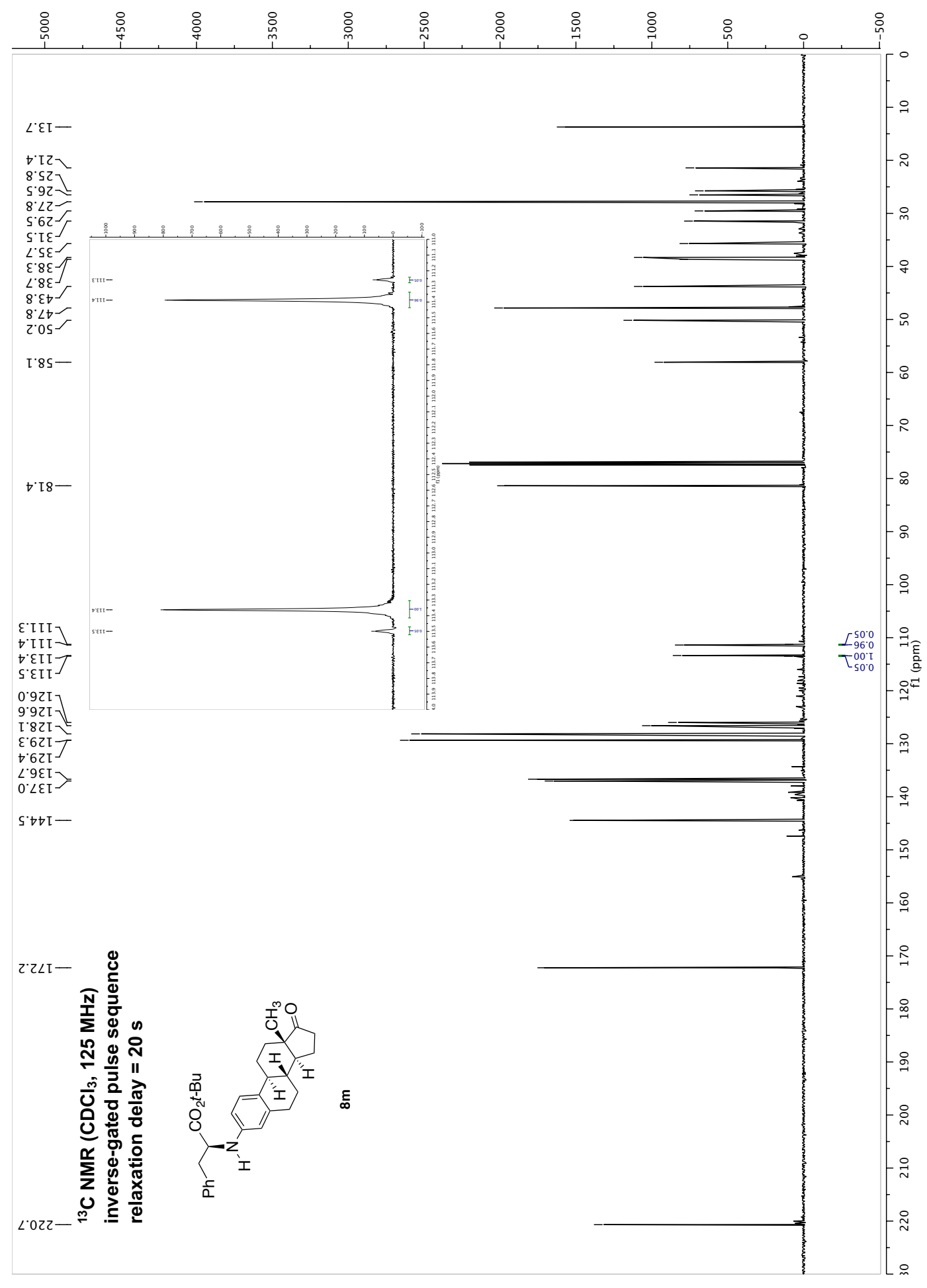




\section{B) Catalog of HPLC Spectra}<smiles>CCOC(=O)C(Cc1ccccc1)Nc1ccccc1</smiles>

$6 a$

HPLC analysis (OJ-H, 2\% IPA-hexanes, $0.8 \mathrm{~mL} / \mathrm{min}, 254 \mathrm{~nm})$ indicated $94 \%$ ee: tR (minor) $=$ $14.4 \mathrm{~min}$, tR (major) $=19.0 \mathrm{~min}$.

\section{DL-6a}

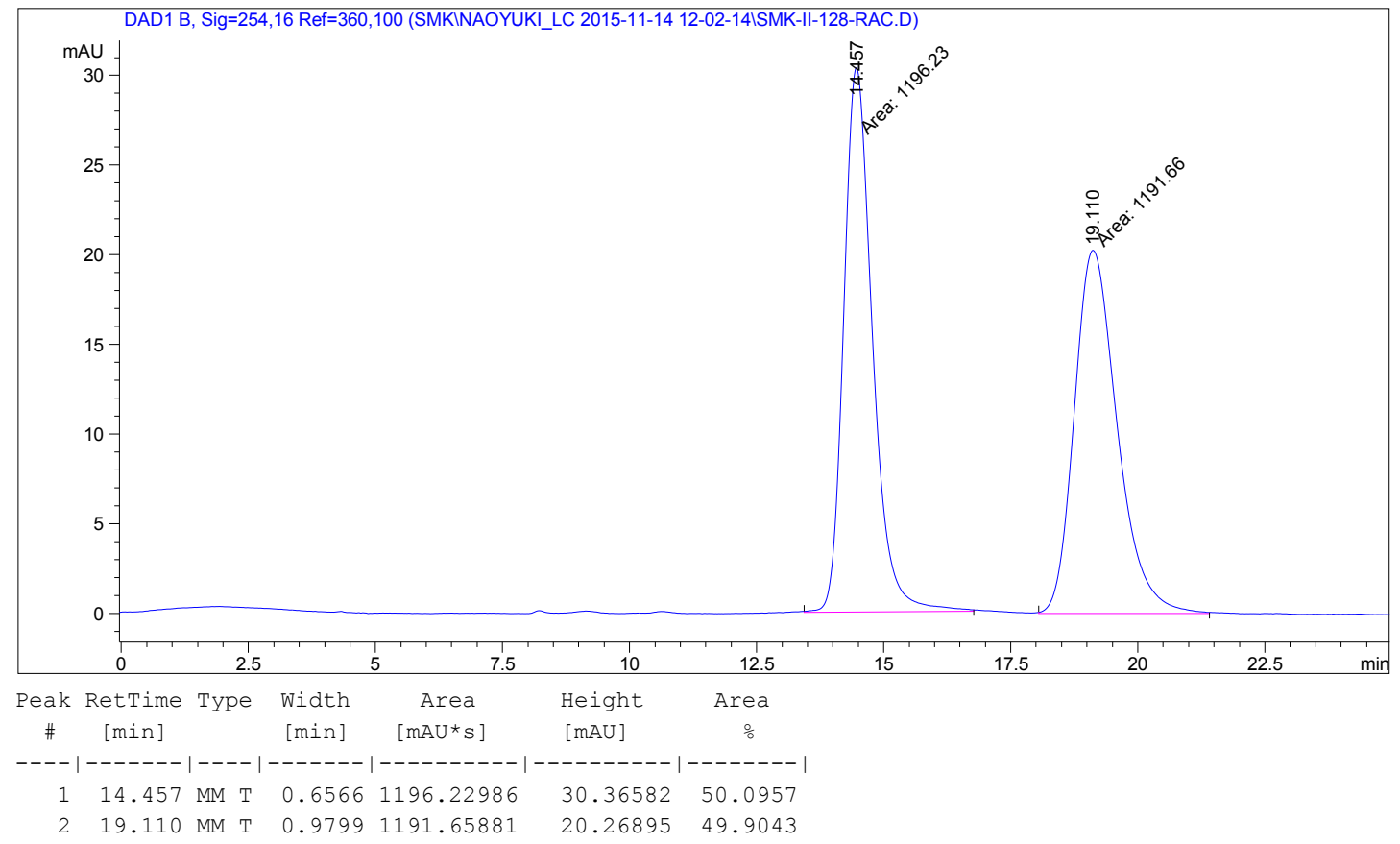

L-6a: $94 \%$ ee

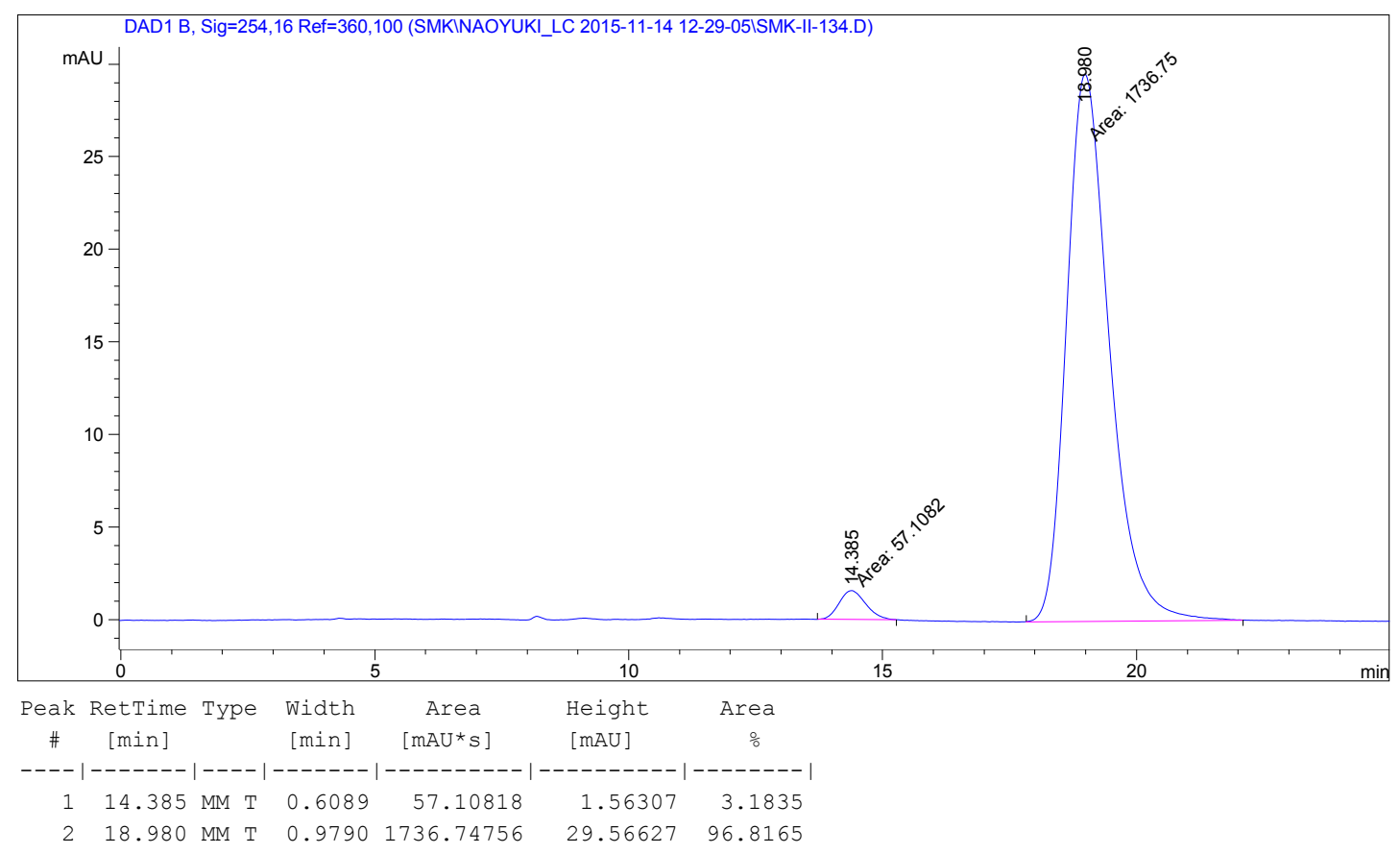


${ }_{\mathrm{NHPh}}^{\mathrm{Me}} \mathrm{CO}_{2} \mathrm{Me}$

$6 c$

HPLC analysis (OJ-H, 10\% IPA-hexanes, $1.0 \mathrm{~mL} / \mathrm{min}, 254 \mathrm{~nm})$ indicated 95\% ee: $\mathrm{tR}(\mathrm{minor})=$ $16.2 \mathrm{~min}, \mathrm{tR}$ (major) $=24.2 \mathrm{~min}$.

\section{DL-6c}

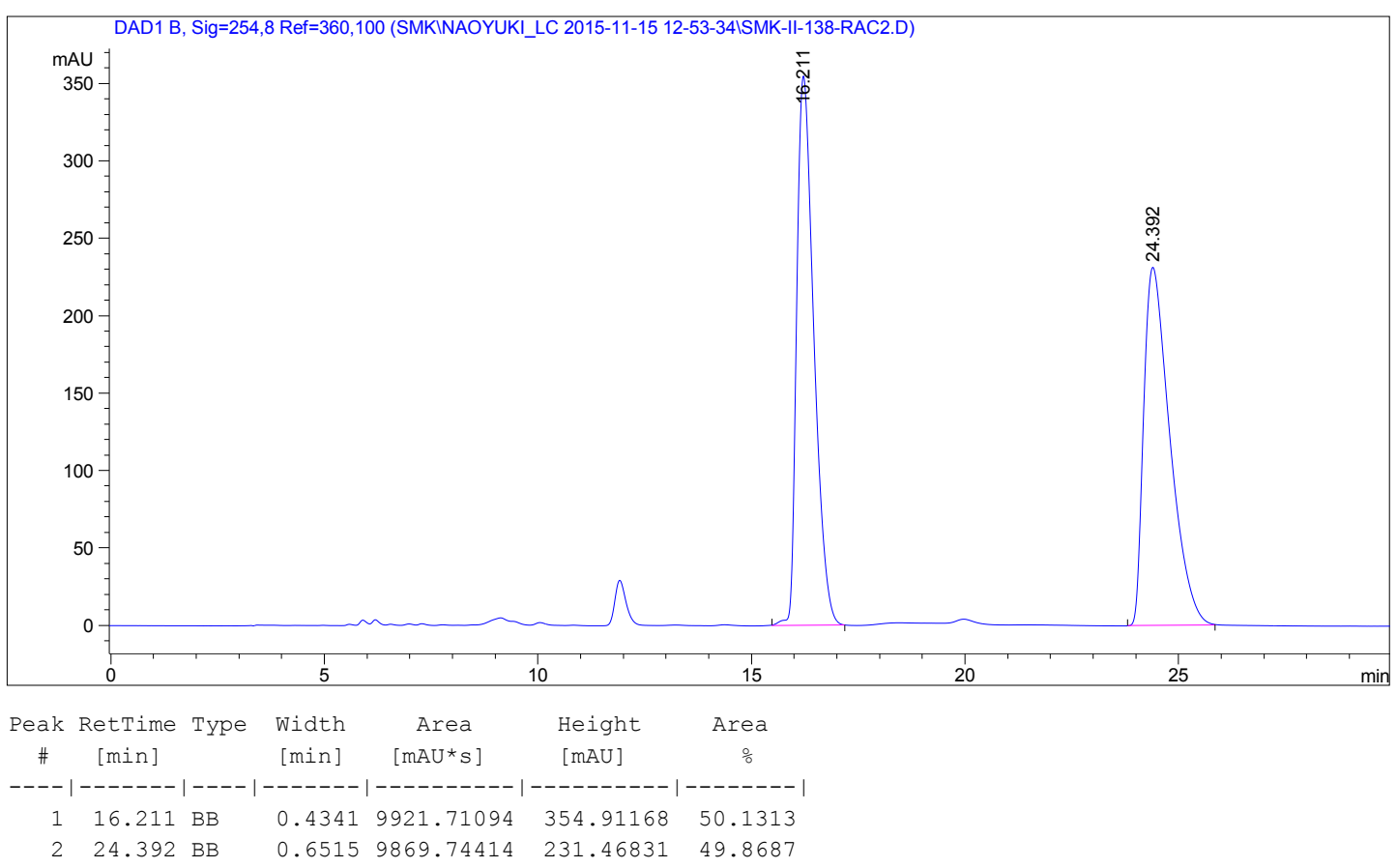

L-6c: $95 \%$ ee

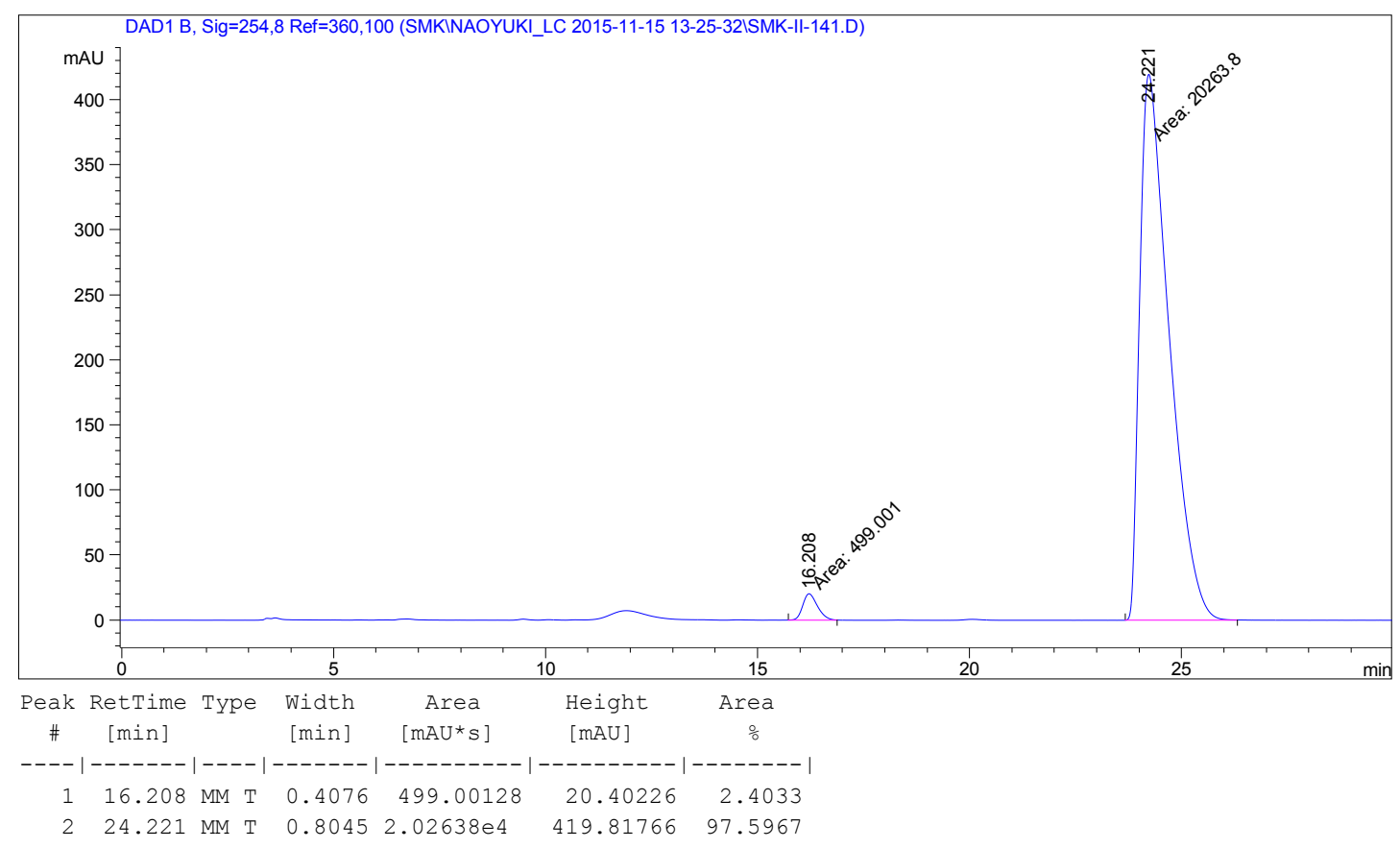


$\mathrm{Me} \underbrace{\mathrm{CO}_{2} t-\mathrm{Bu}}_{\mathrm{NHPh}}$

$6 d$

HPLC analysis (OJ-H, 5\% IPA-hexanes, $0.8 \mathrm{~mL} / \mathrm{min}, 254 \mathrm{~nm})$ indicated $96 \%$ ee: $\mathrm{tR}$ (minor) = $8.7 \mathrm{~min}, \mathrm{tR}$ (major) $=9.3 \mathrm{~min}$.

\section{DL-6d}

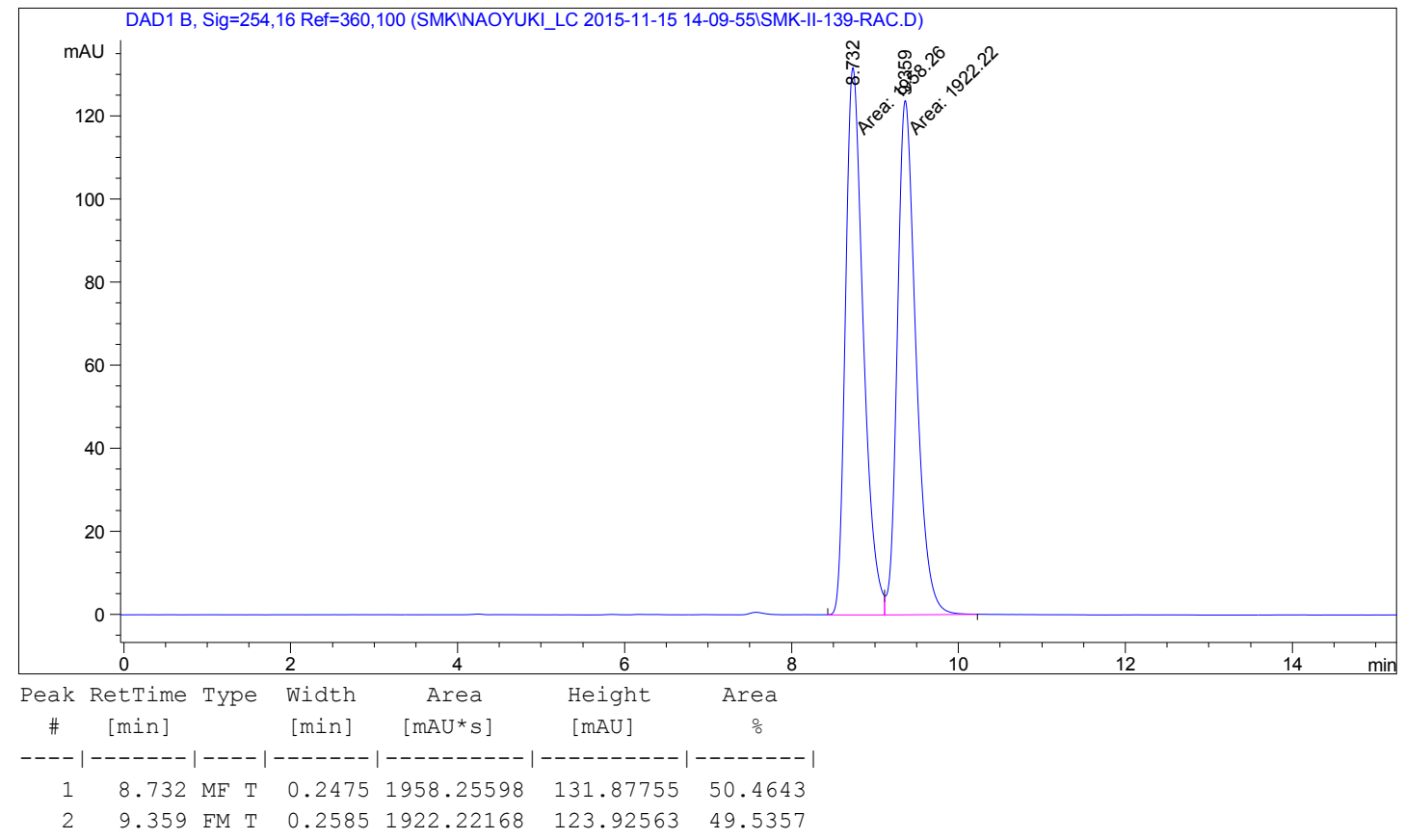

L-6d: 96\% ee

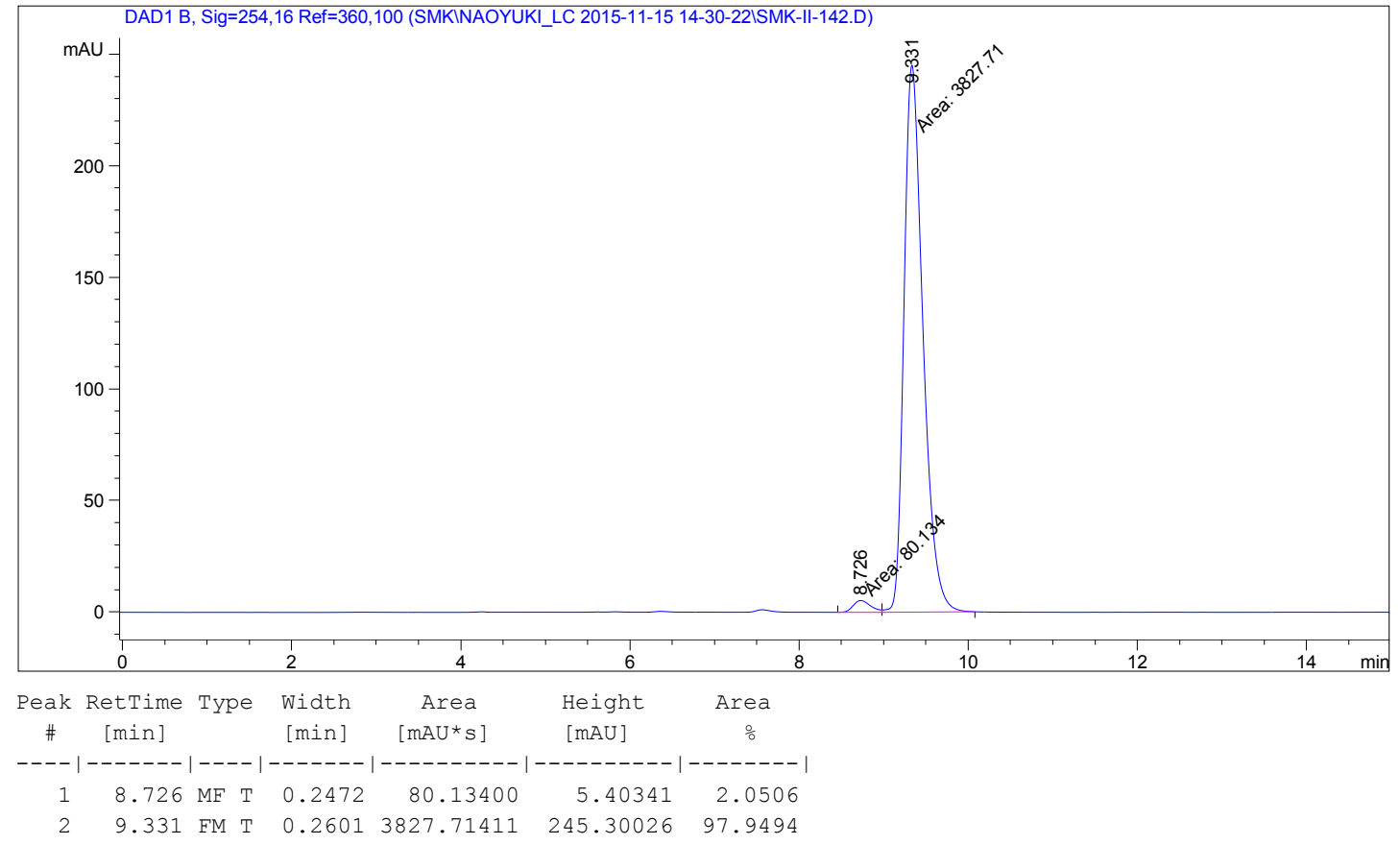


$\overbrace{\mathrm{NHPh}}^{\mathrm{Me}} \mathrm{CO}_{2} t-\mathrm{Bu}$

6e

HPLC analysis (OJ-H, 0.5\% IPA-hexanes, $0.5 \mathrm{~mL} / \mathrm{min}, 254 \mathrm{~nm})$ indicated $87 \%$ ee: $\mathrm{tR}$ (major) = $12.3 \mathrm{~min}, \mathrm{tR}($ minor $)=13.3 \mathrm{~min}$.

\section{DL-6e}

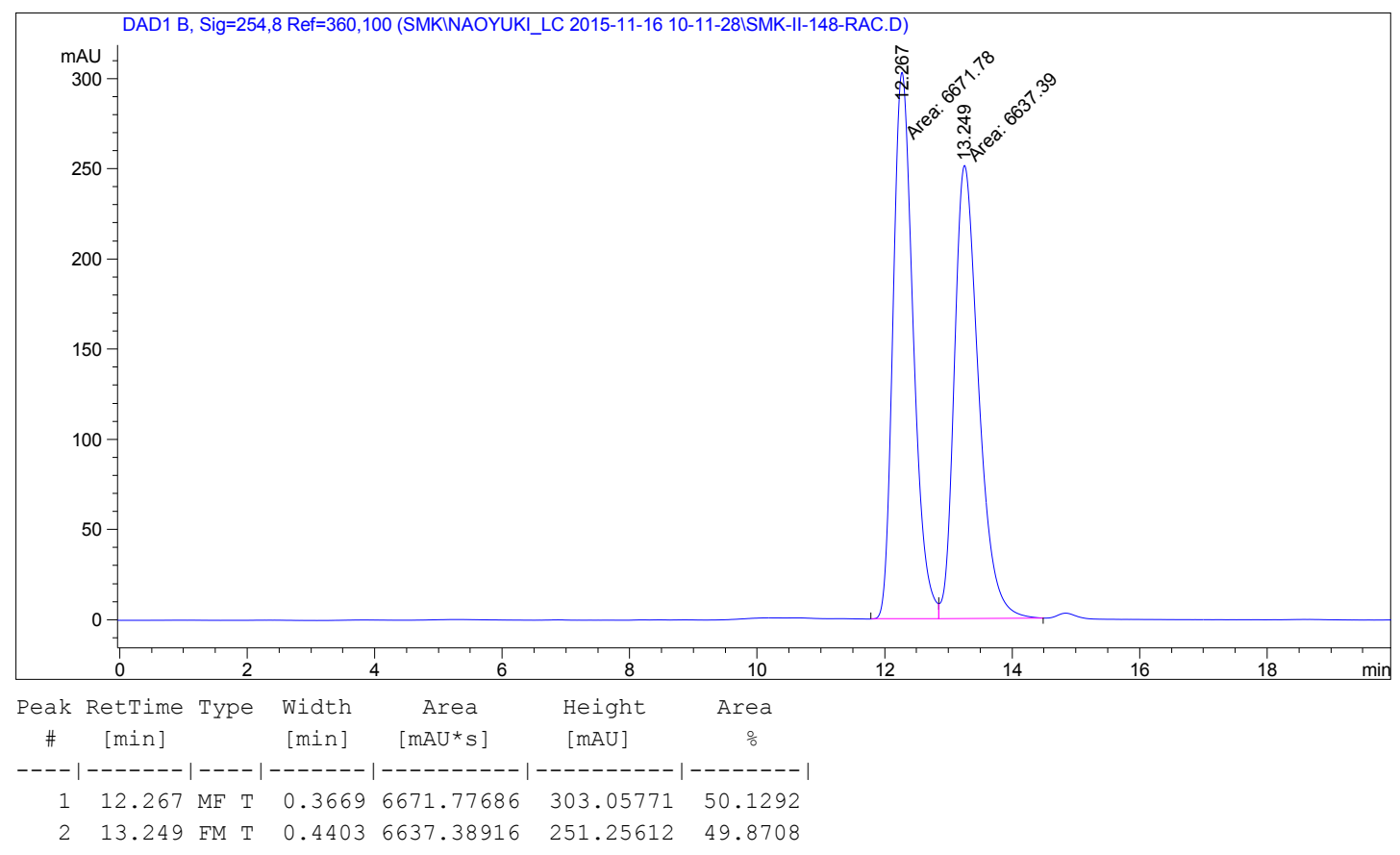

L-6e: $87 \%$ ee

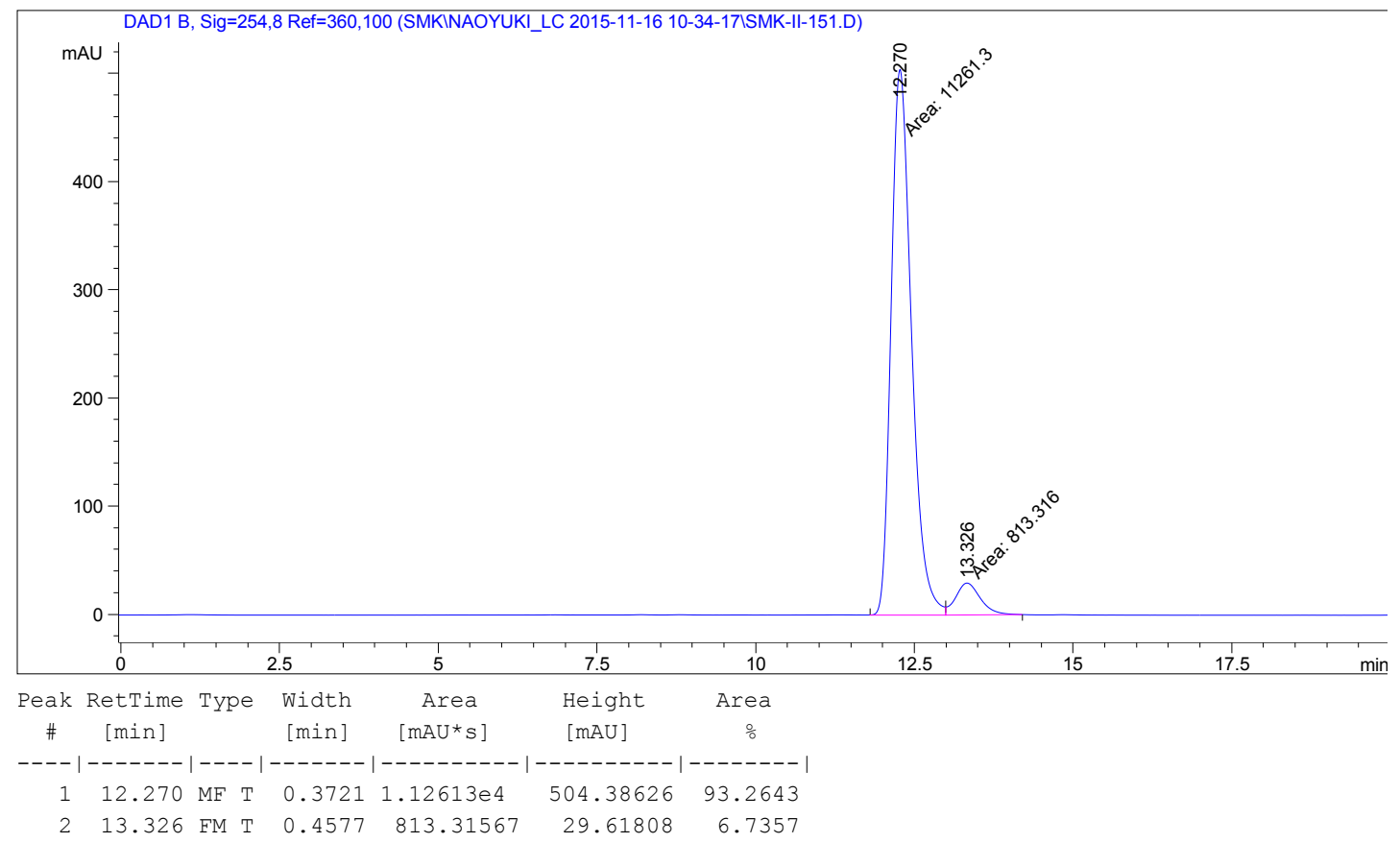


$\overbrace{\mathrm{NHPh}}^{\mathrm{Me}} \mathrm{CO}_{2} \mathrm{Bn}$

6f

HPLC analysis (OJ-H, 10\% IPA-hexanes, $1.0 \mathrm{~mL} / \mathrm{min}, 254 \mathrm{~nm})$ indicated $94 \%$ ee: $\mathrm{tR}($ minor $)=$ $19.0 \mathrm{~min}$, tR (major) $=24.0 \mathrm{~min}$.

\section{DL-6f}

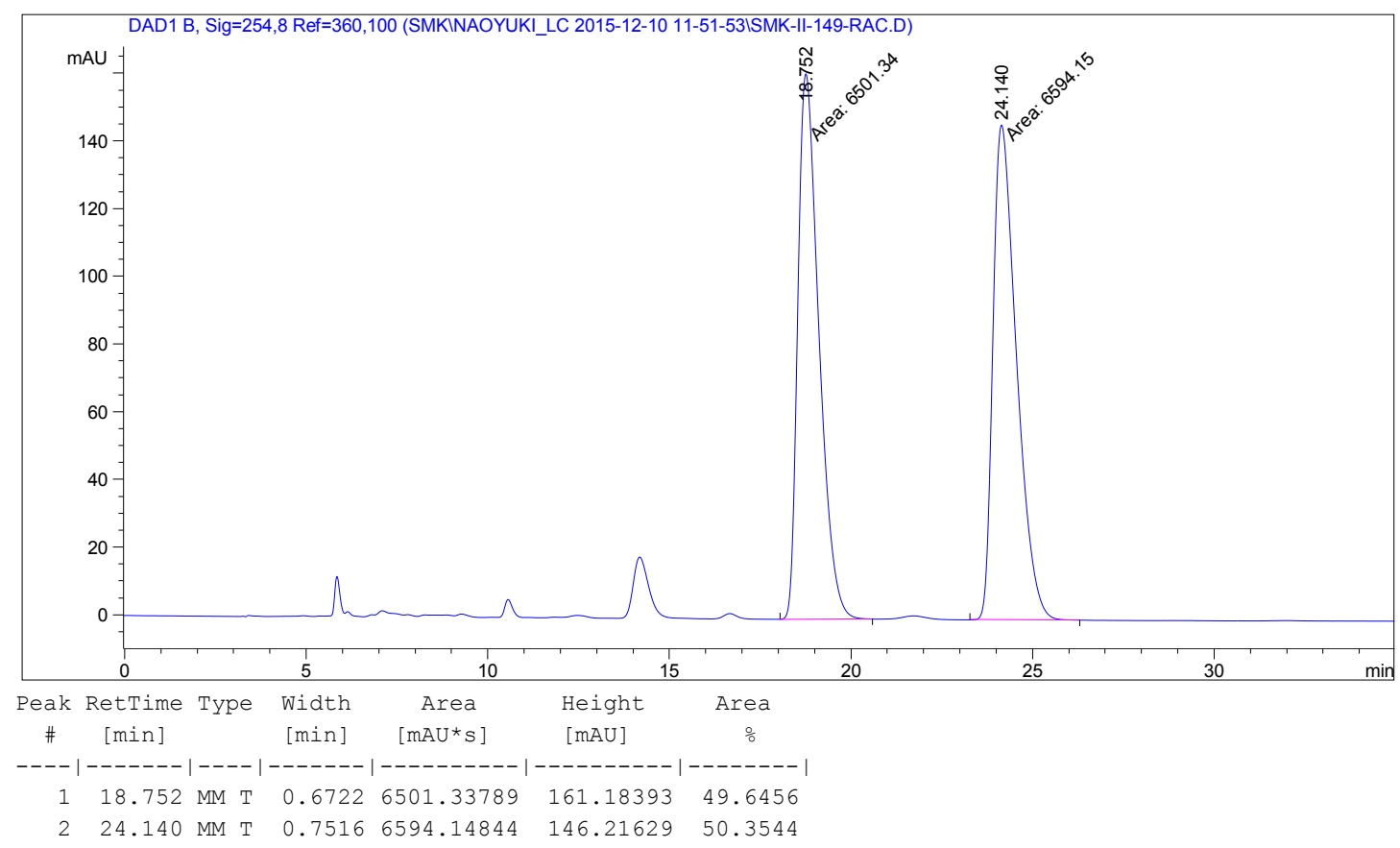

\section{L-6f: $94 \%$ ee}

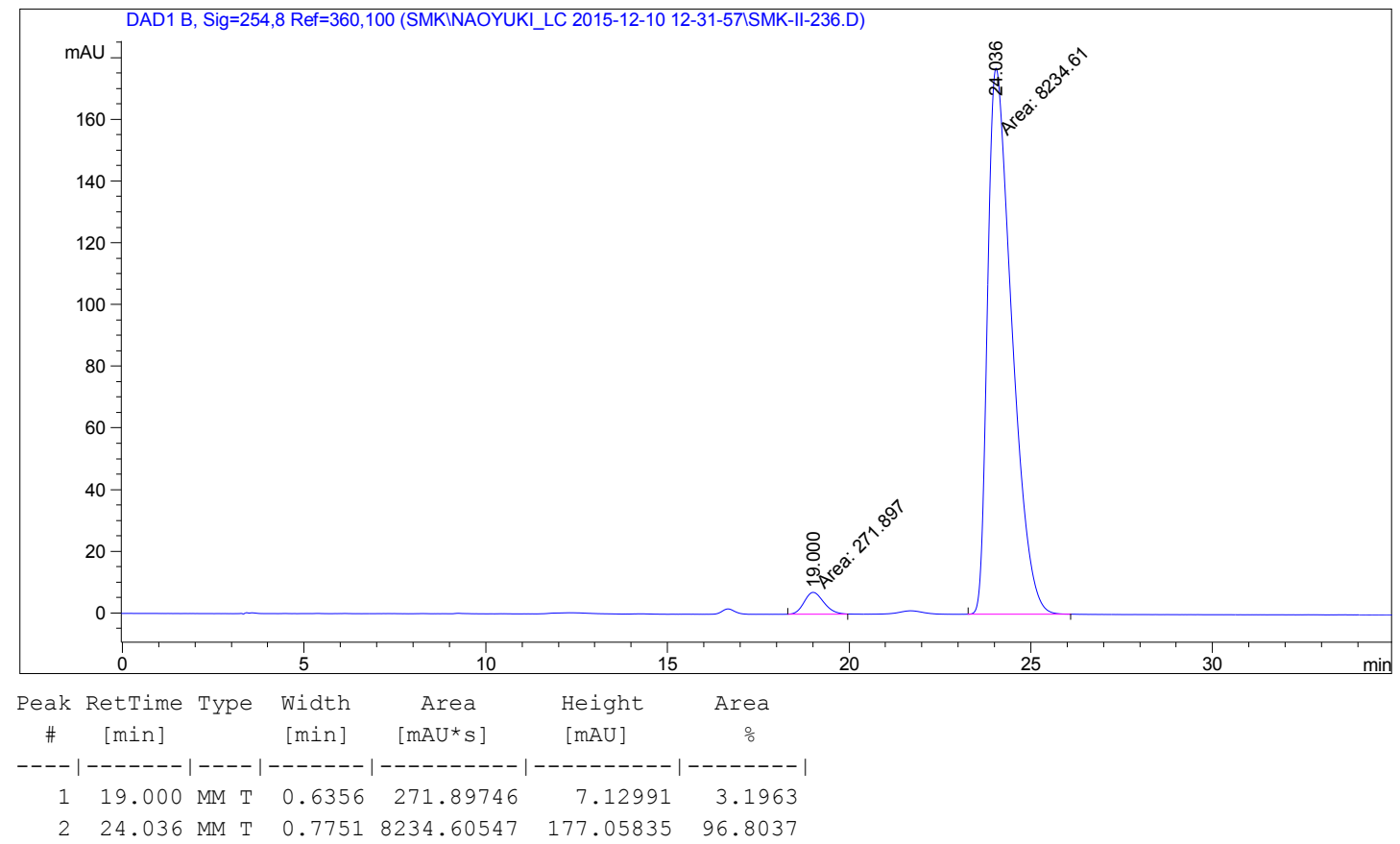




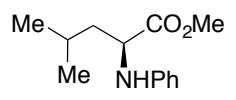

$6 \mathrm{~g}$

HPLC analysis (OJ-H, 5\% IPA-hexanes, $0.8 \mathrm{~mL} / \mathrm{min}, 254 \mathrm{~nm})$ indicated $96 \%$ ee: tR (minor) $=$ $10.2 \mathrm{~min}, \mathrm{tR}$ (major) $=15.0 \mathrm{~min}$.

\section{DL-6g}

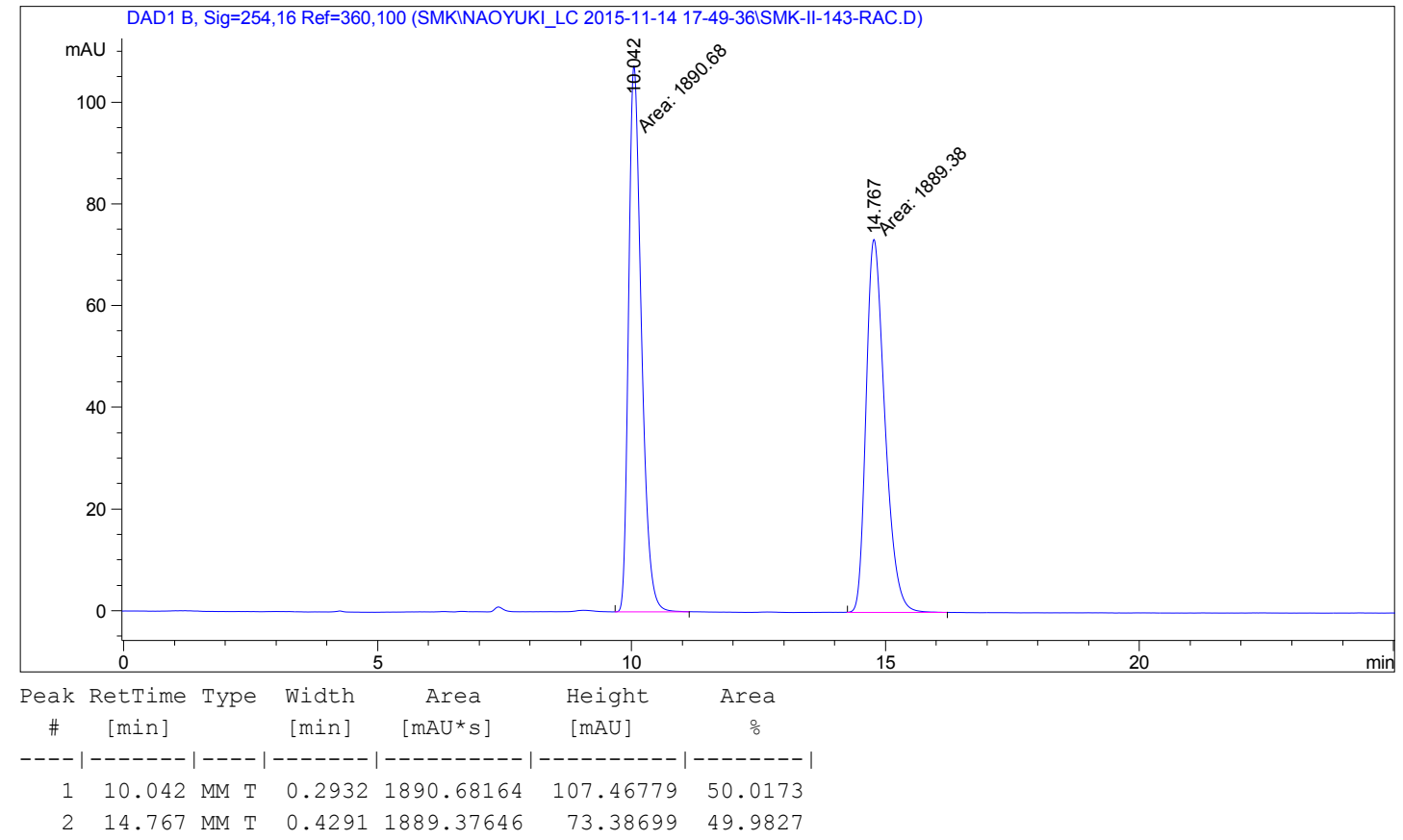

L-6g: $96 \%$ ee

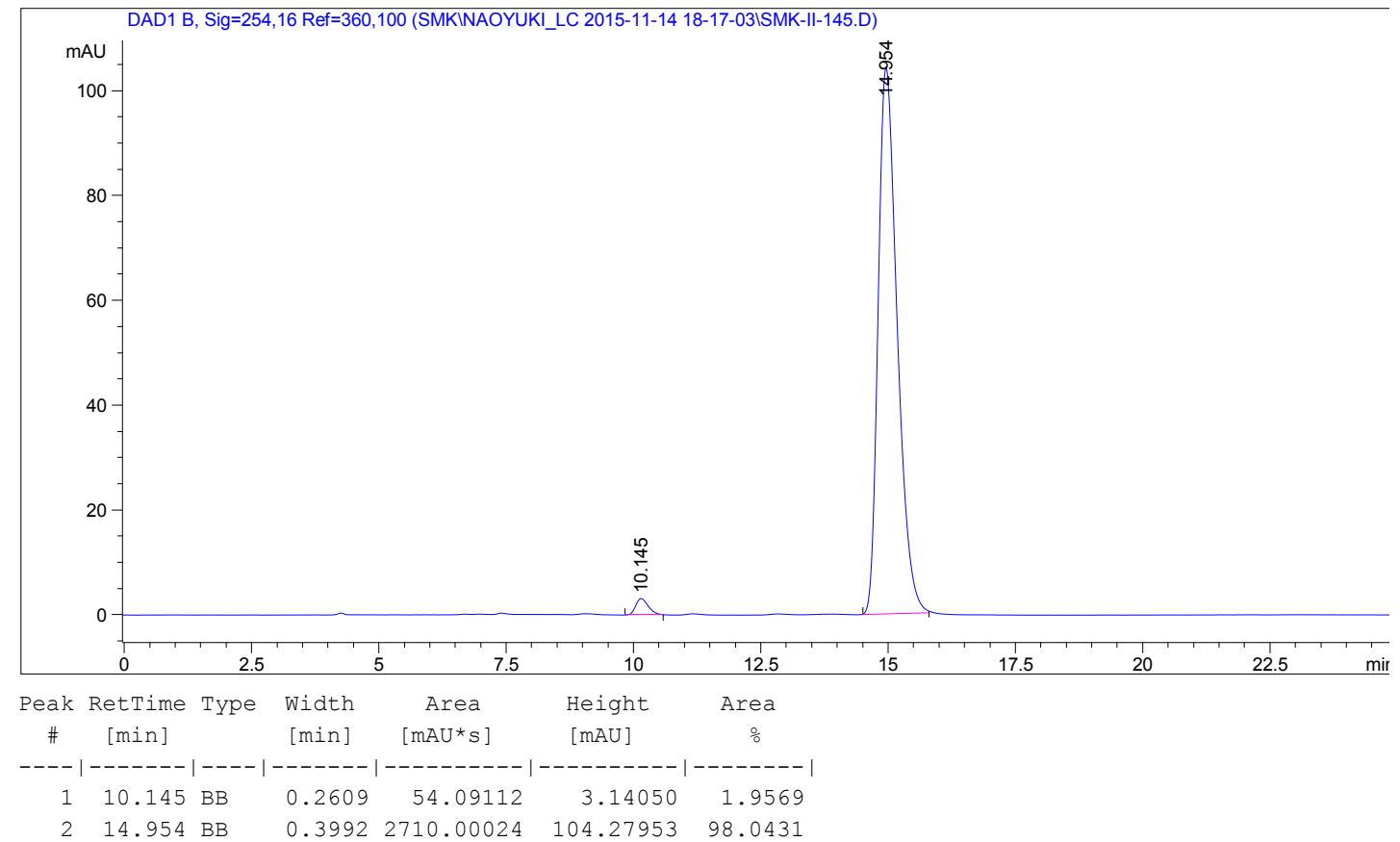


$\underbrace{\mathrm{Me}}_{\mathrm{Me}} \mathrm{CO}_{2} \mathrm{t}-\mathrm{Bu}$

$6 \mathrm{~h}$

HPLC analysis (OJ-H, 1\% IPA-hexanes, $0.8 \mathrm{~mL} / \mathrm{min}, 254 \mathrm{~nm}$ ) indicated 97\% ee: tR (minor) = $6.3 \mathrm{~min}, \mathrm{tR}$ (major) $=7.0 \mathrm{~min}$.

\section{DL-6h}

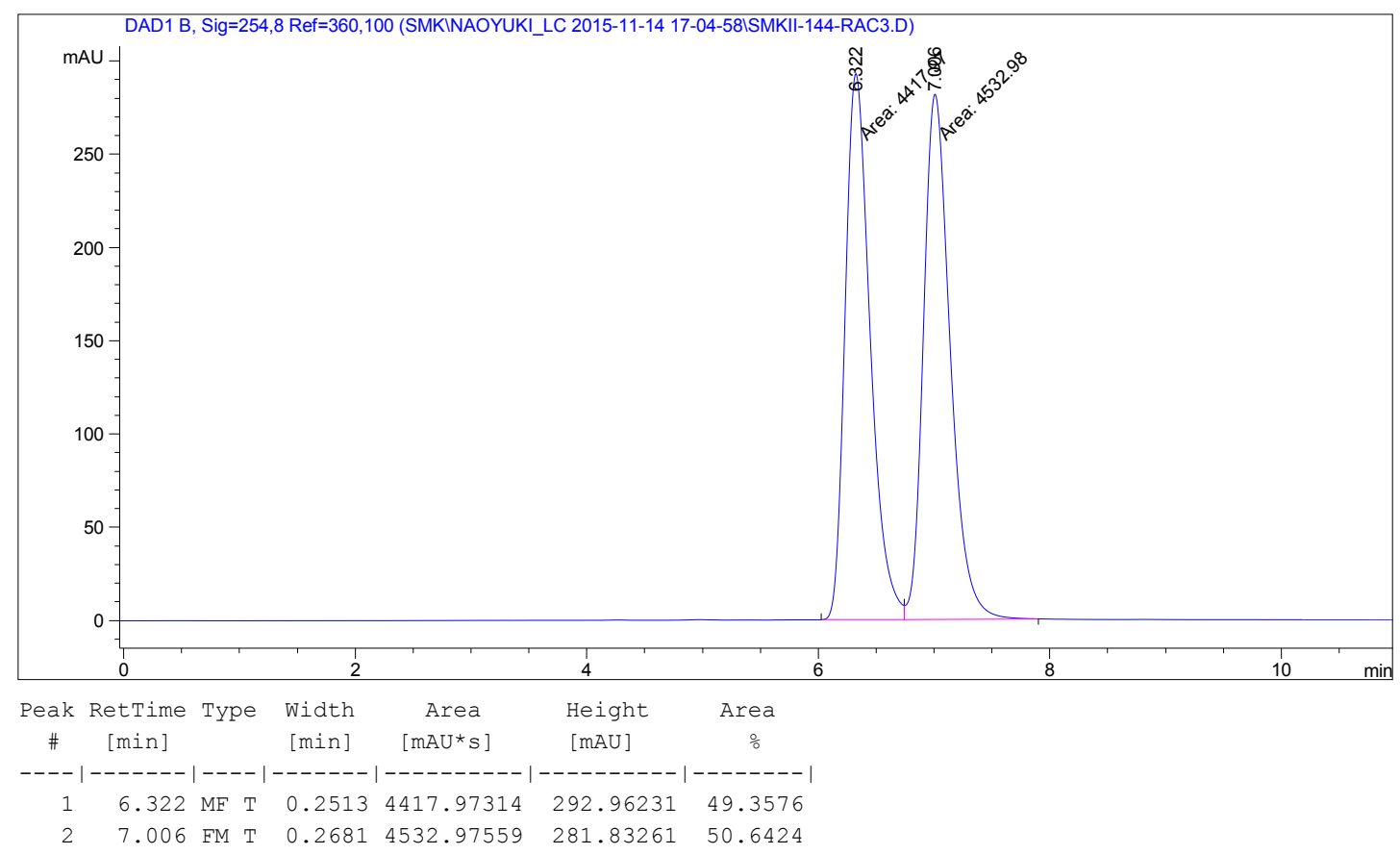

L-6h: $97 \%$ ee

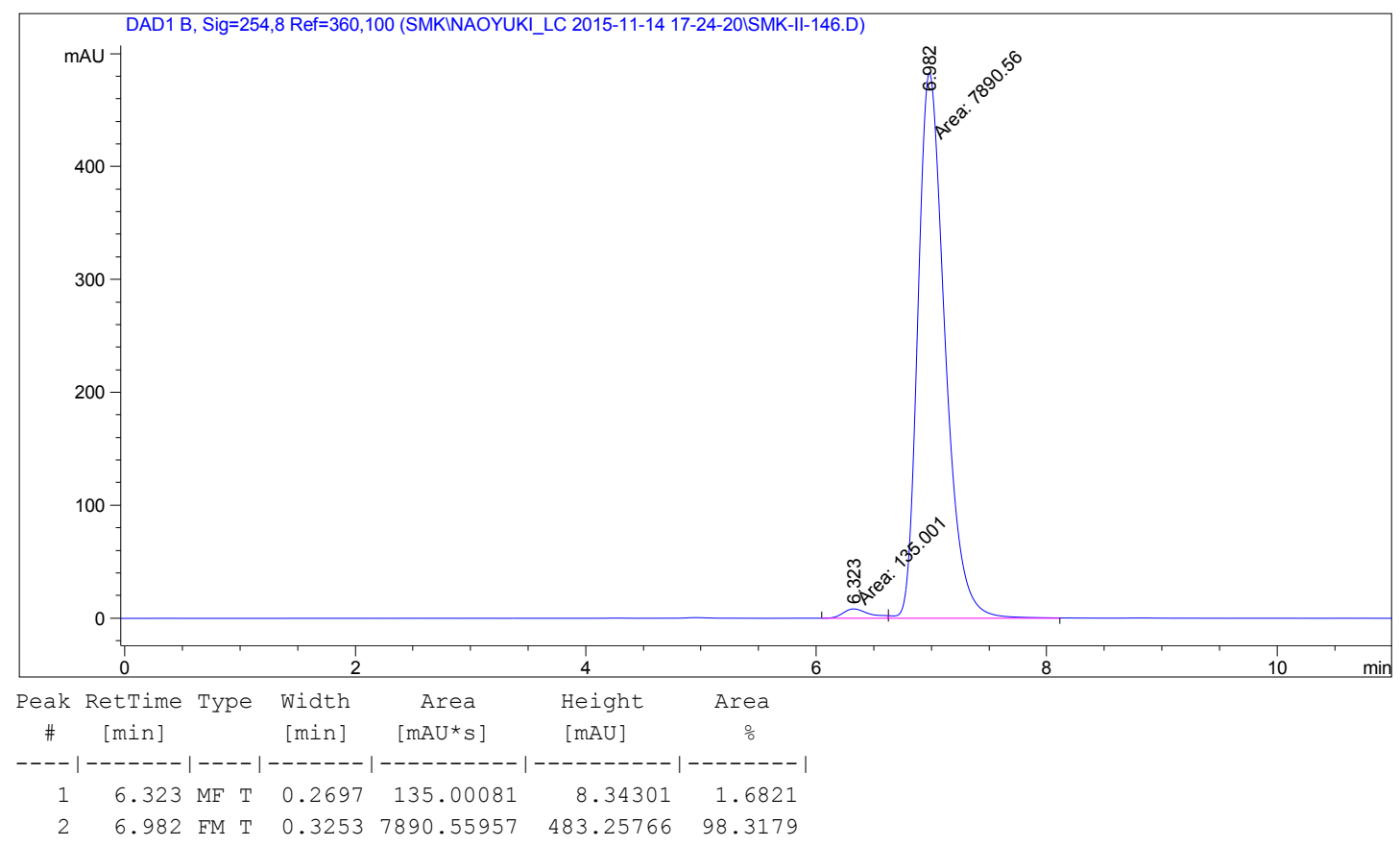


$\mathrm{Ph} \overbrace{\mathrm{NHPh}}^{\mathrm{CO}_{2} \mathrm{Me}}$

$6 i$

HPLC analysis (OJ-H, 10\% IPA-hexanes, $1.0 \mathrm{~mL} / \mathrm{min}, 254 \mathrm{~nm})$ indicated $87 \%$ ee: tR (minor) = $30.7 \mathrm{~min}$, tR (major) $=42.8 \mathrm{~min}$.

\section{DL-6i}

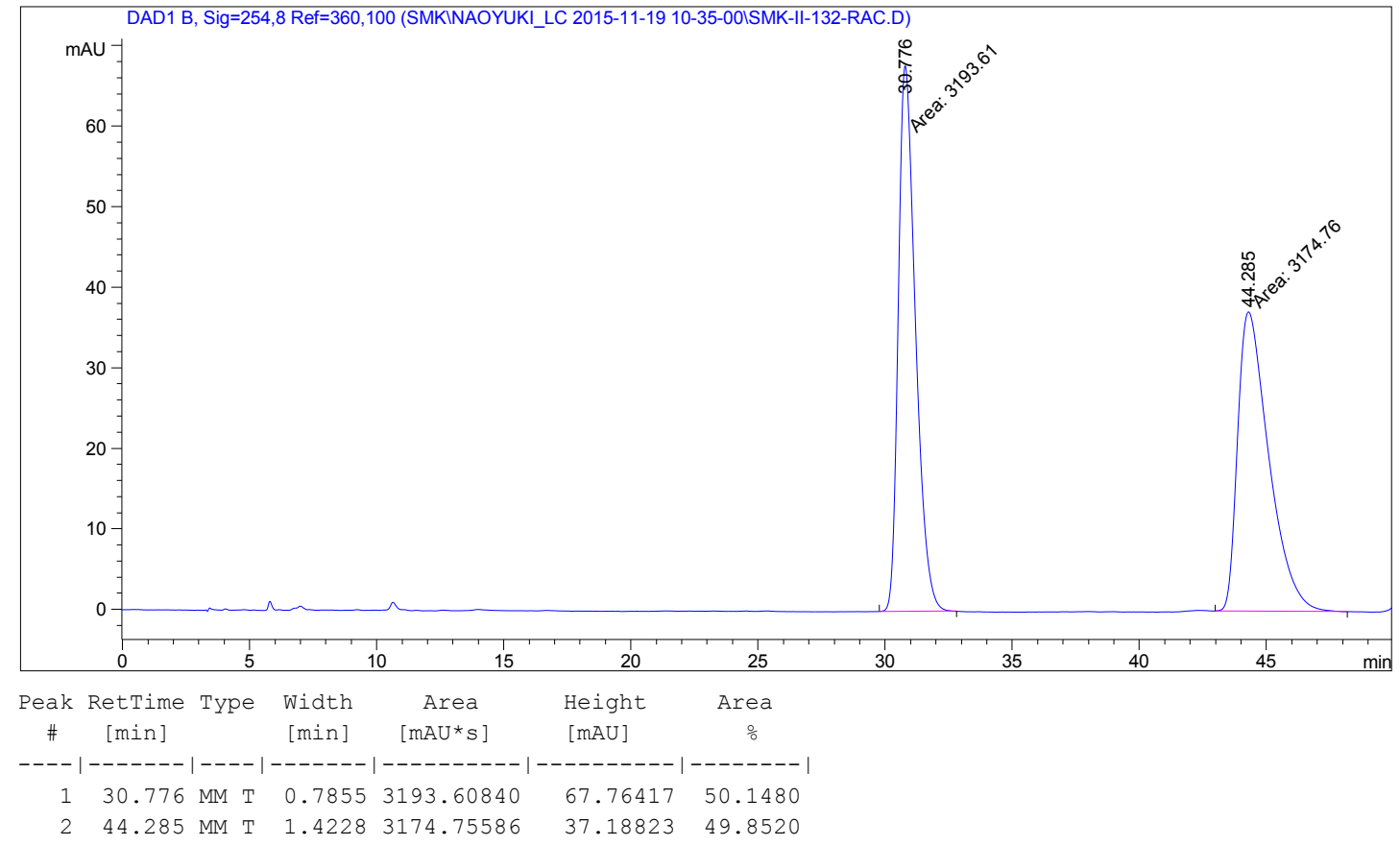

L-6i: $87 \%$ ee

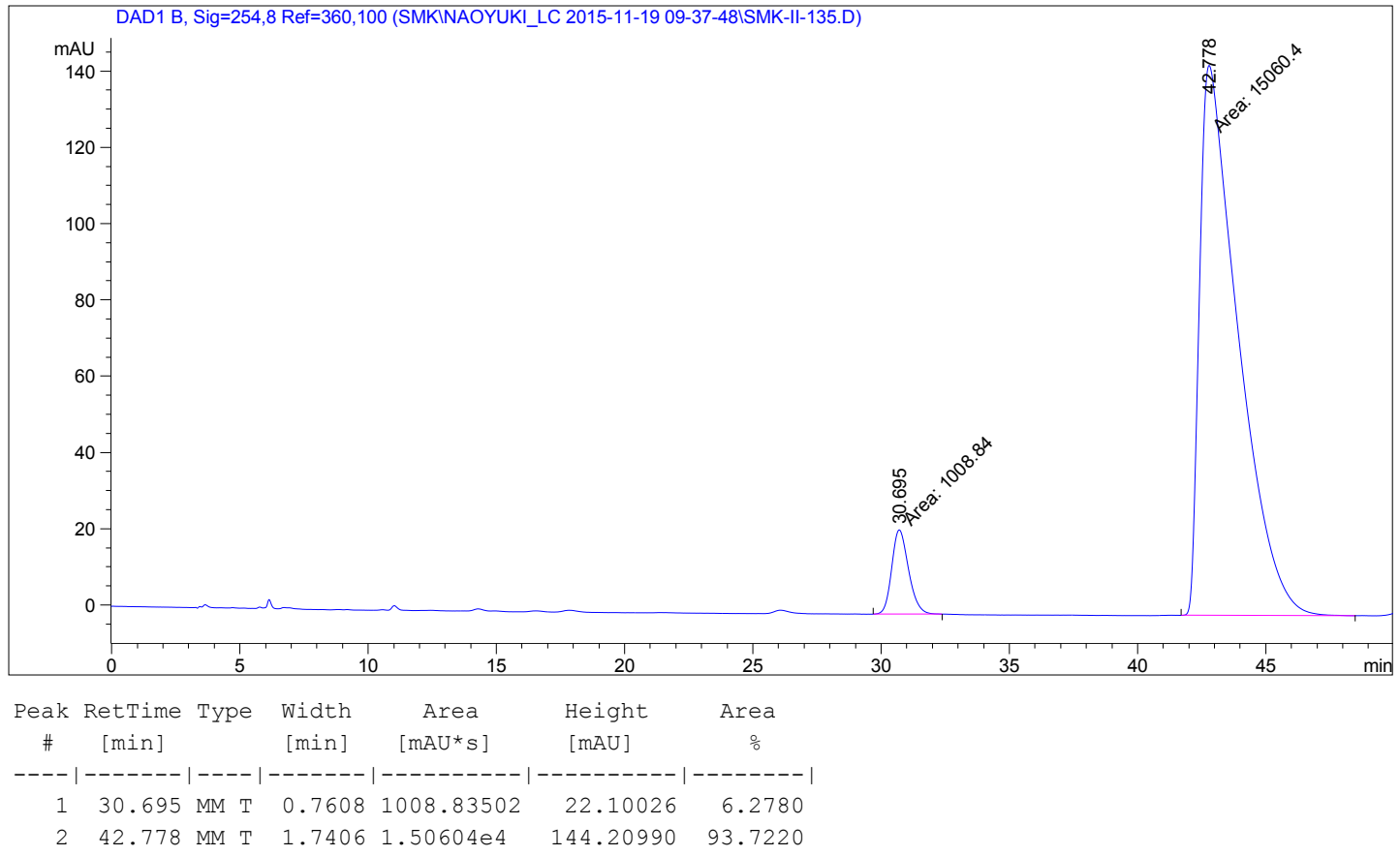




6j

HPLC analysis (OD-H, 10\% IPA-hexanes, $1.0 \mathrm{~mL} / \mathrm{min}, 254 \mathrm{~nm})$ indicated 96\% ee: $\mathrm{tR}(\mathrm{major})=$ $29.8 \mathrm{~min}, \mathrm{tR}$ (minor) $=54.4 \mathrm{~min}$.

\section{DL-6j}

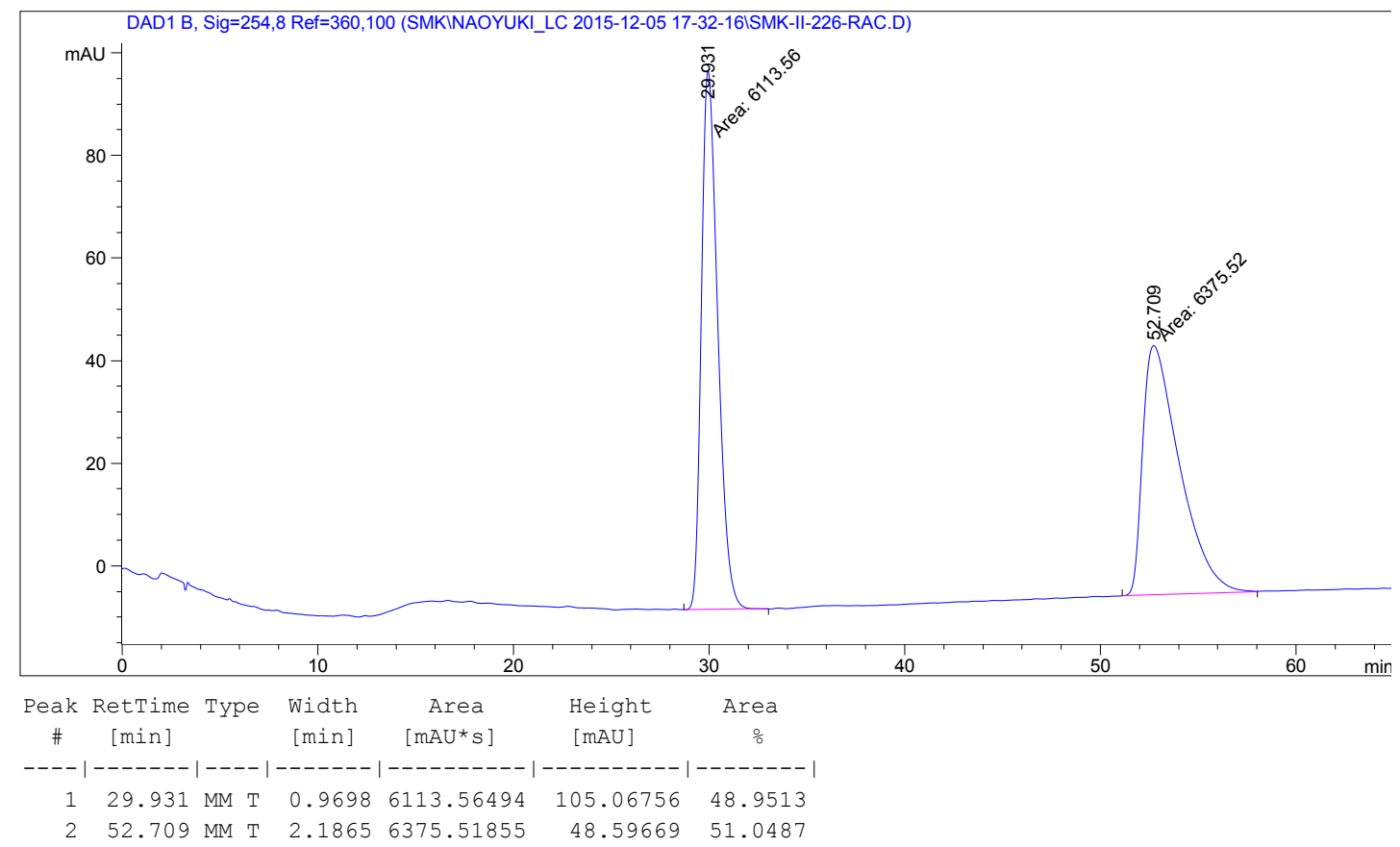

L-6j: $96 \%$ ee

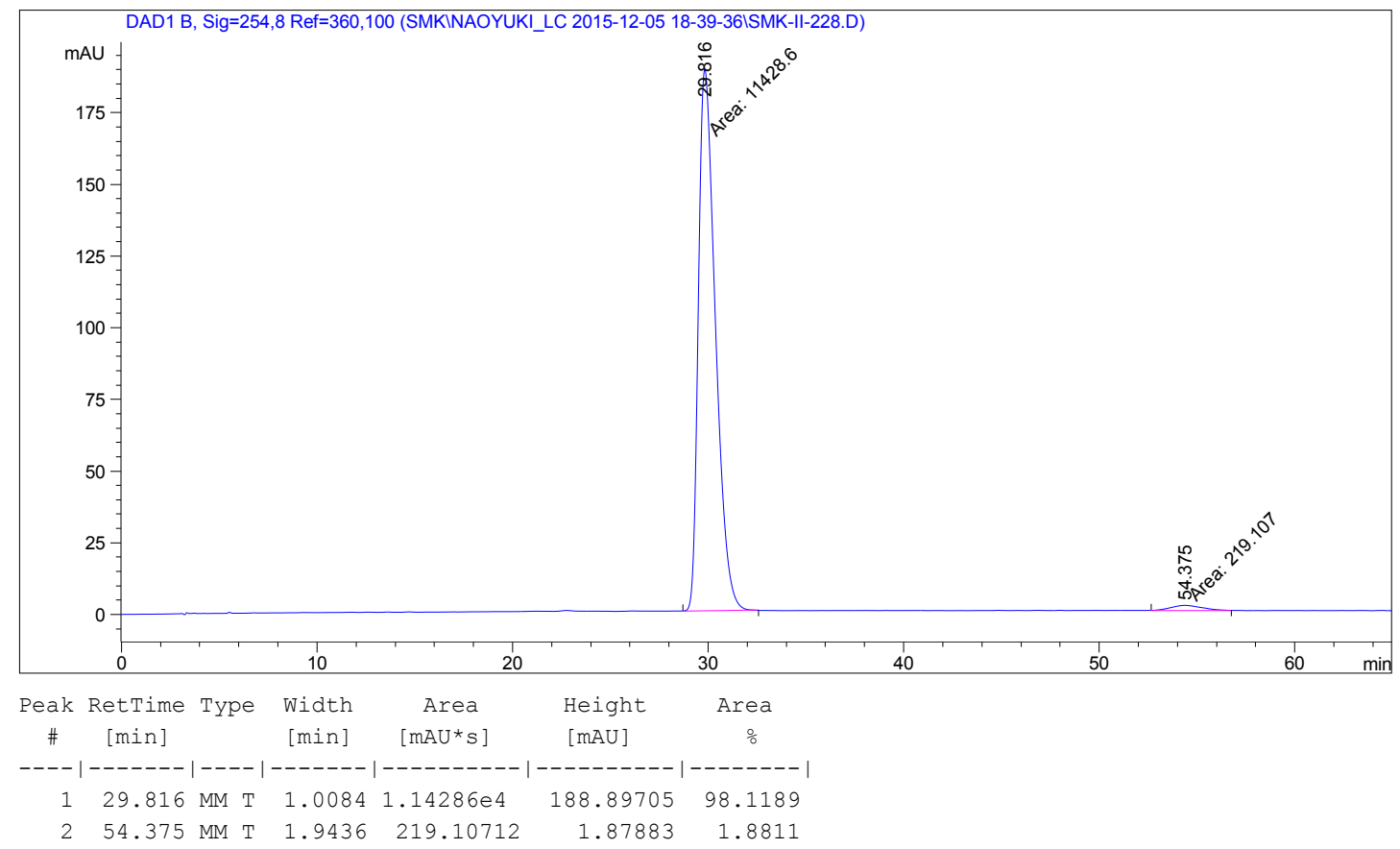




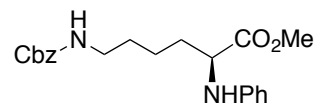

$6 k$

HPLC analysis (OD-H, 20\% IPA-hexanes, $1.0 \mathrm{~mL} / \mathrm{min}, 254 \mathrm{~nm}$ ) indicated $94 \%$ ee: tR (major) = $19.7 \mathrm{~min}, \mathrm{tR}$ (minor) $=40.3 \mathrm{~min}$.

\section{DL-6k}

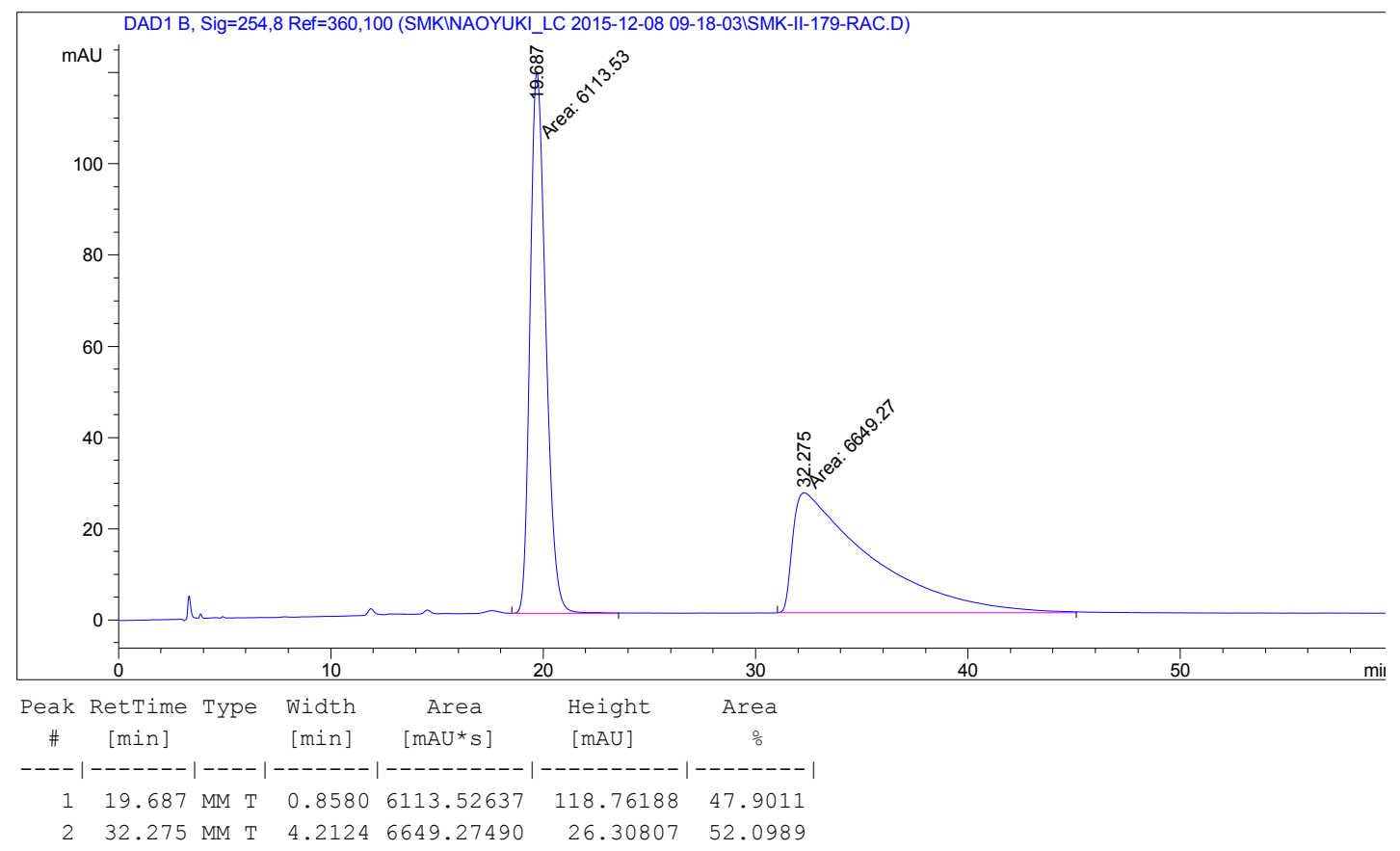

L-6k: 94\%

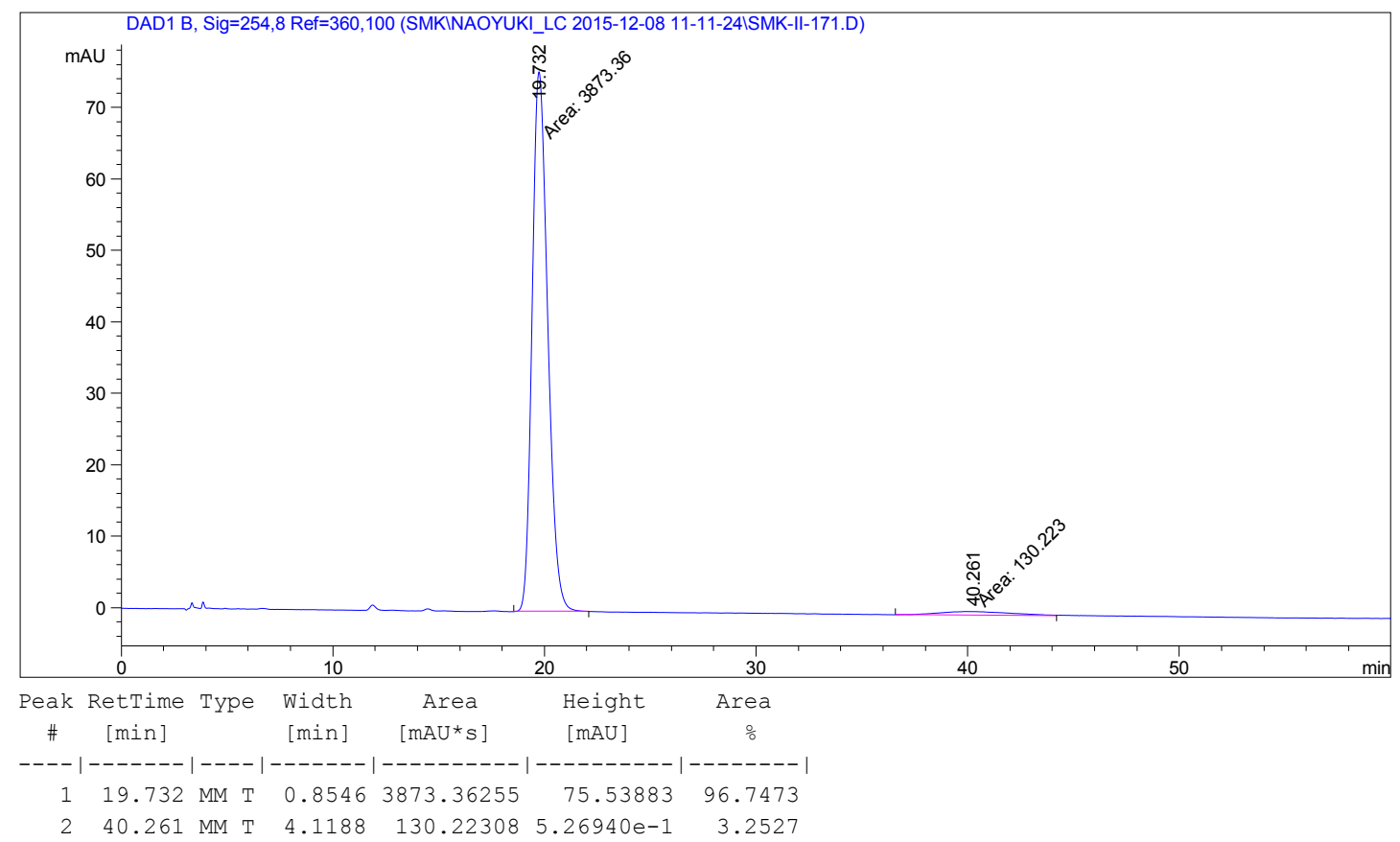


$\underbrace{\mathrm{CO}_{2} \mathrm{Me}}_{\mathrm{NHPh}}$

61

HPLC analysis (AD-H, 10\% IPA-hexanes, $1.0 \mathrm{~mL} / \mathrm{min}, 254 \mathrm{~nm}$ ) indicated $92 \%$ ee: $\mathrm{tR}$ (minor) = $10.5 \mathrm{~min}$, tR (major) $=30.1 \mathrm{~min}$.

\section{DL-61}

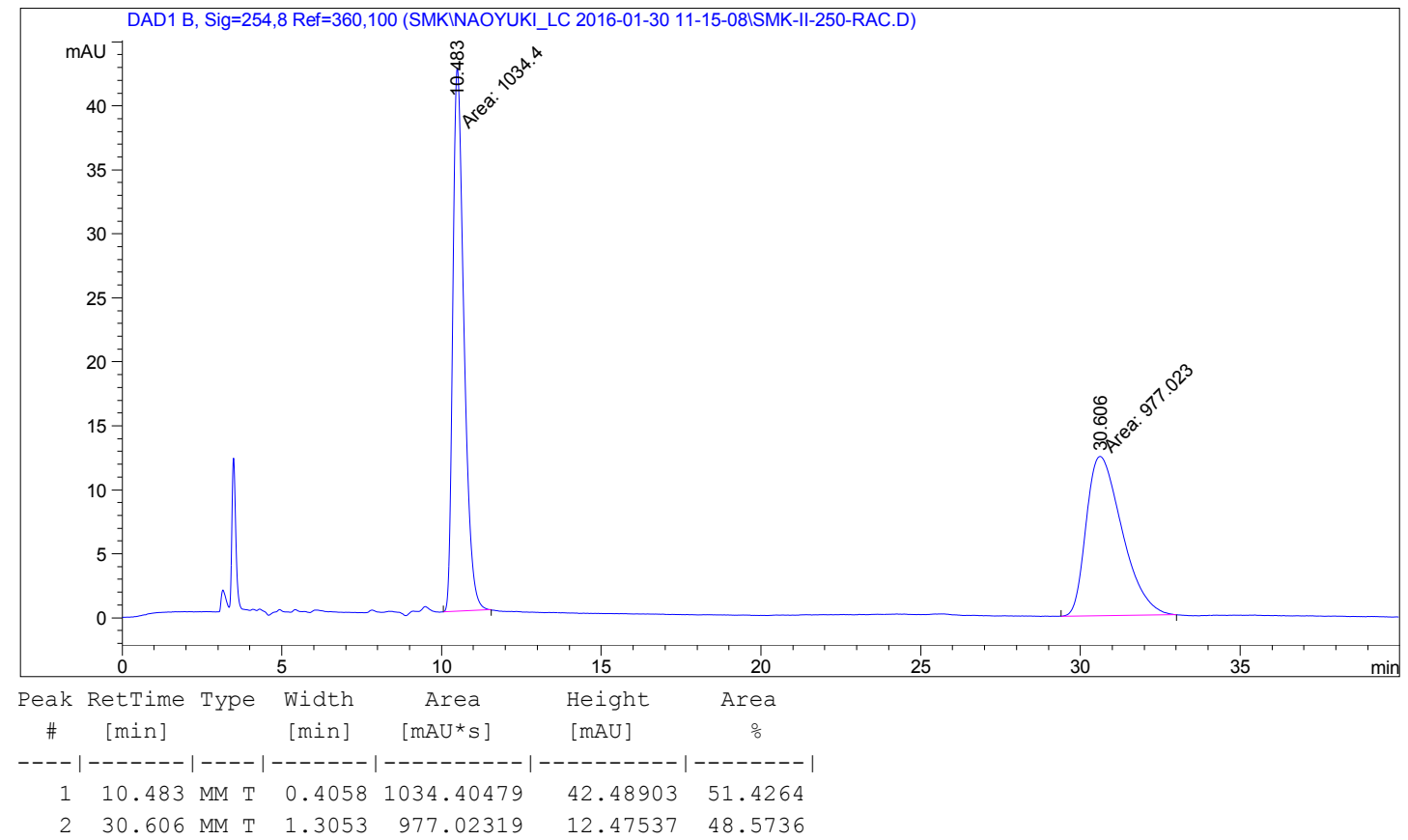

L-61: $92 \%$

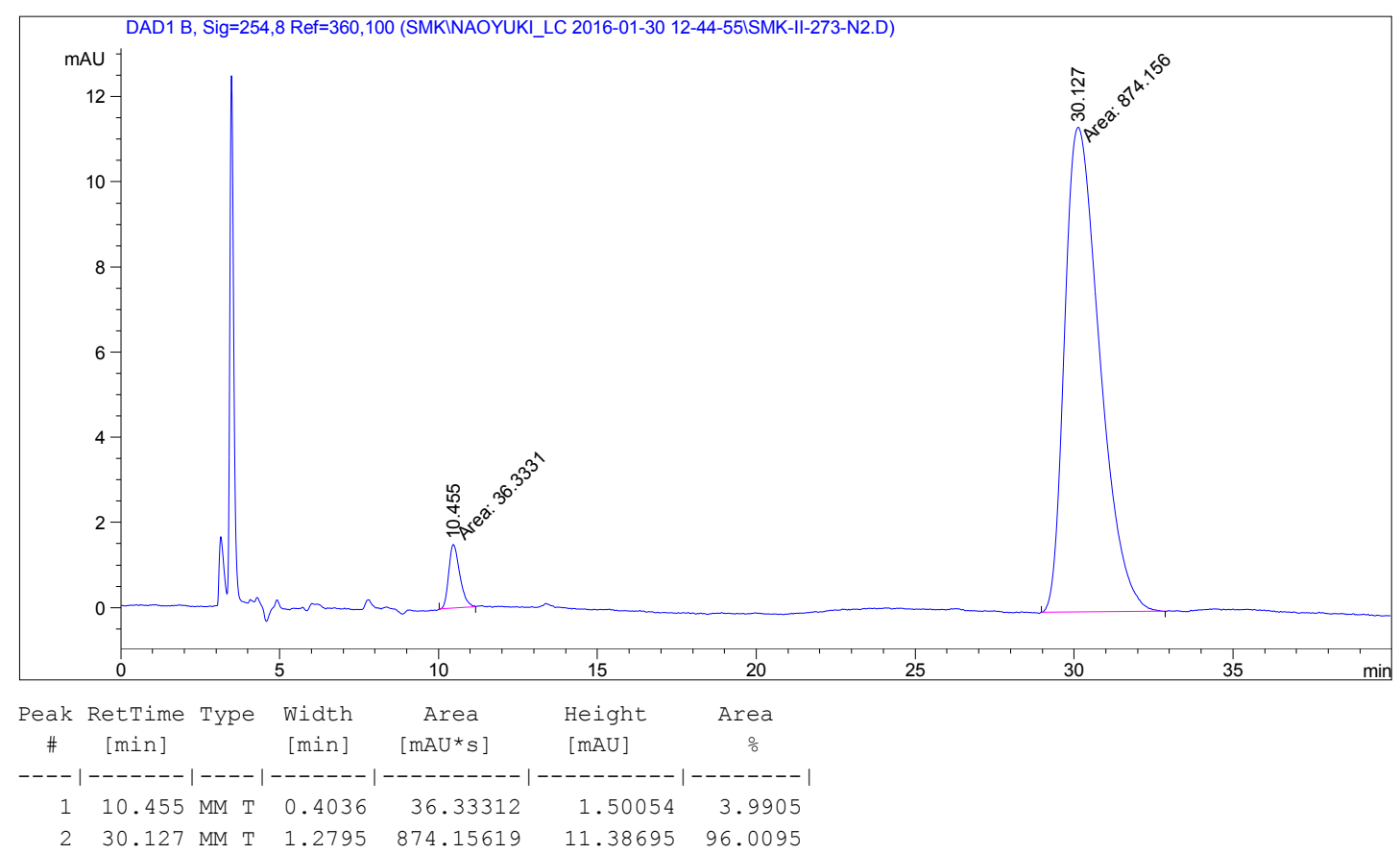




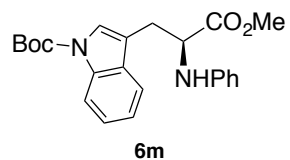

HPLC analysis (OJ-H, 10\% IPA-hexanes, $1.0 \mathrm{~mL} / \mathrm{min}, 254 \mathrm{~nm})$ indicated 93\% ee: tR (major) = $13.4 \mathrm{~min}, \mathrm{tR}($ minor $)=16.7 \mathrm{~min}$.

\section{DL-6m}

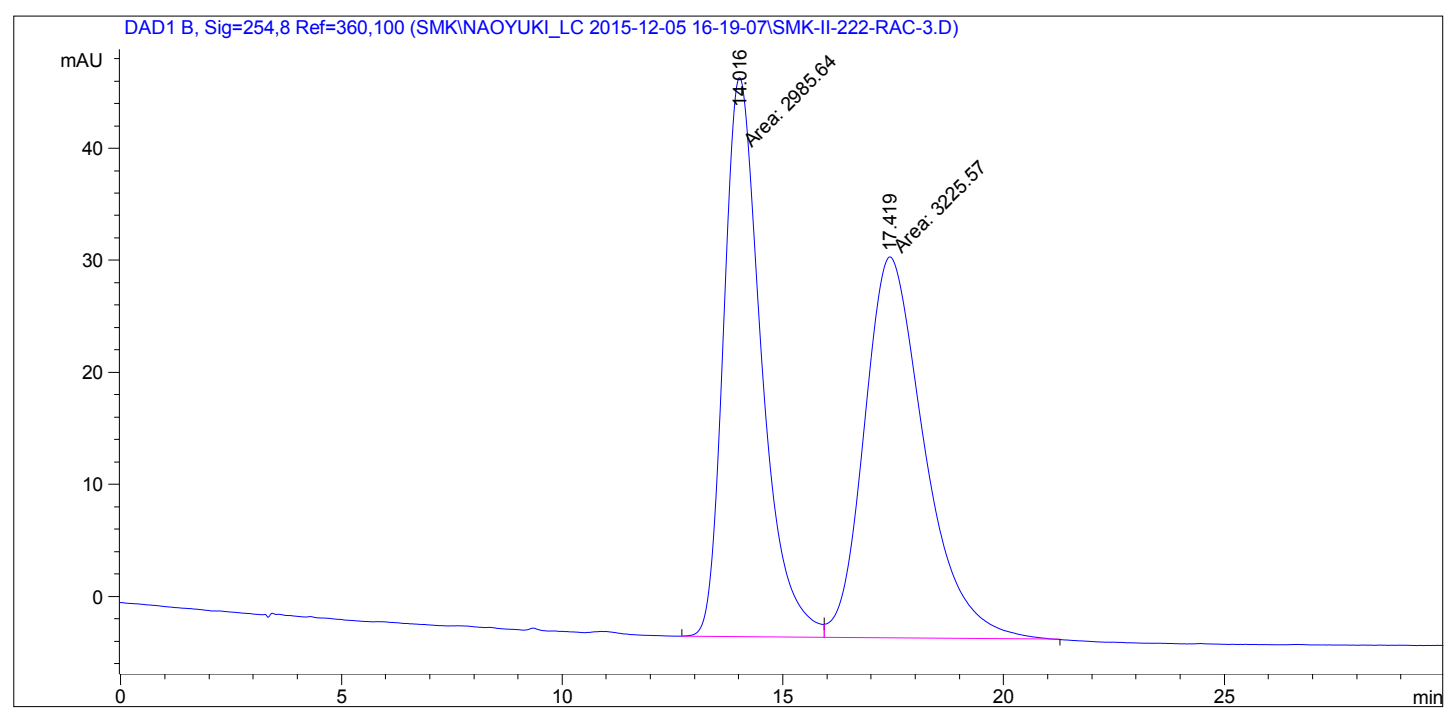

\begin{tabular}{|c|c|c|c|c|c|c|}
\hline $\begin{array}{c}\text { Peak } \\
\#\end{array}$ & $\begin{array}{c}\text { RetTime } \\
\text { [min] }\end{array}$ & Type & $\begin{array}{l}\text { Width } \\
\text { [min] }\end{array}$ & $\begin{array}{c}\text { Area } \\
{\left[\mathrm{mAU}^{*} \mathrm{~S}\right]}\end{array}$ & $\begin{array}{l}\text { Height } \\
{[\mathrm{mAU}]}\end{array}$ & $\begin{array}{c}\text { Area } \\
\frac{\circ}{0}\end{array}$ \\
\hline & & & & ---------- & --- & ---1 \\
\hline 1 & & $\mathrm{~T}$ & 5 & 2985.64014 & 3752 & 686 \\
\hline 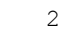 & 17 & $\mathrm{~T}$ & 93 & 3225.56665 & 3899 & 9314 \\
\hline
\end{tabular}

L-6m: 93\% ee

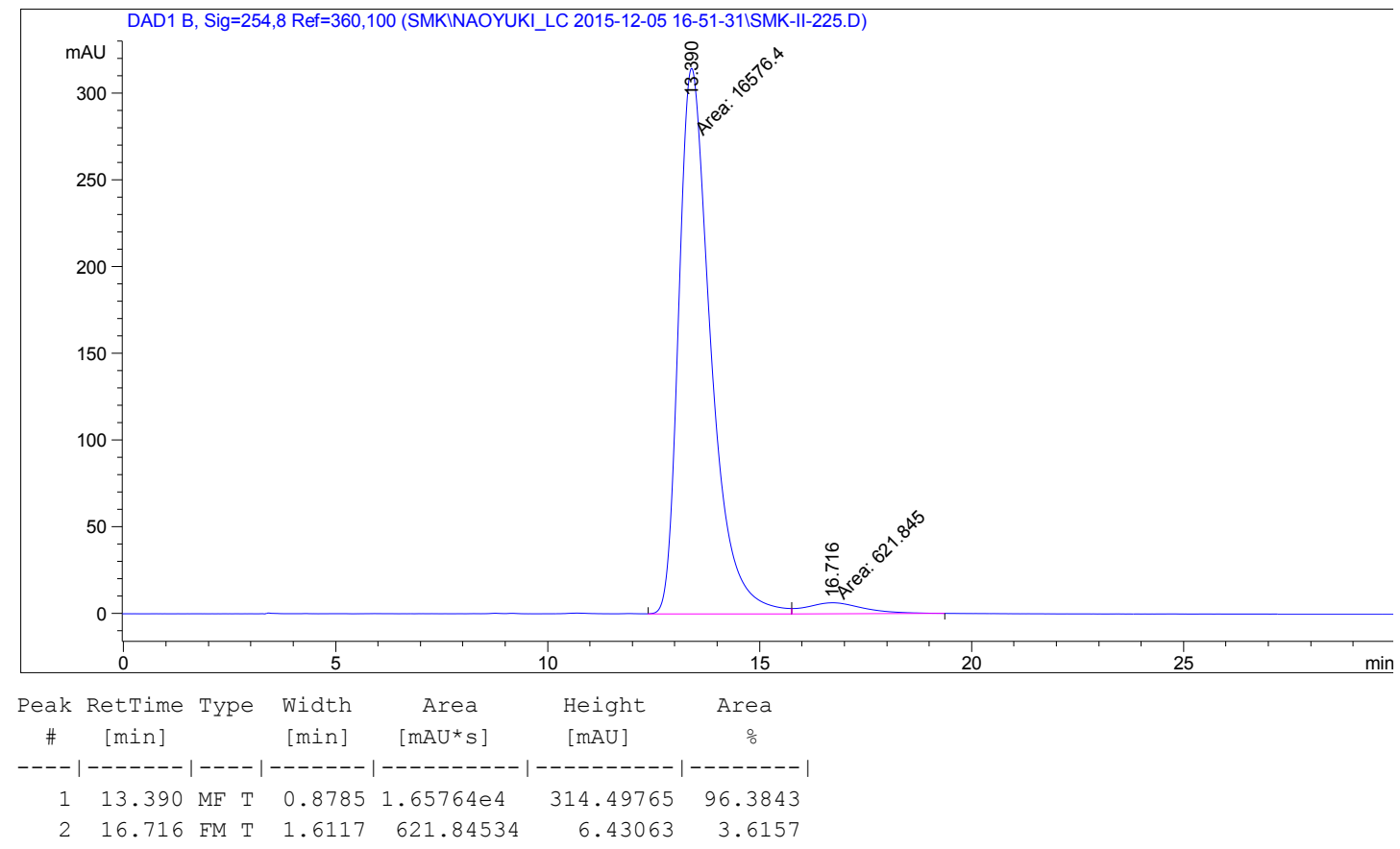


Ph $\underbrace{\mathrm{NHPh}}_{6 n} \mathrm{CO}_{2} \mathrm{Me}$

HPLC analysis (OD-H, 5\% IPA-hexanes, $0.8 \mathrm{~mL} / \mathrm{min}, 254 \mathrm{~nm}$ ) indicated $99 \%$ ee: tR (major) $=$ $19.8 \mathrm{~min}, \mathrm{tR}$ (minor) $=21.6 \mathrm{~min}$.

\section{DL-6n}

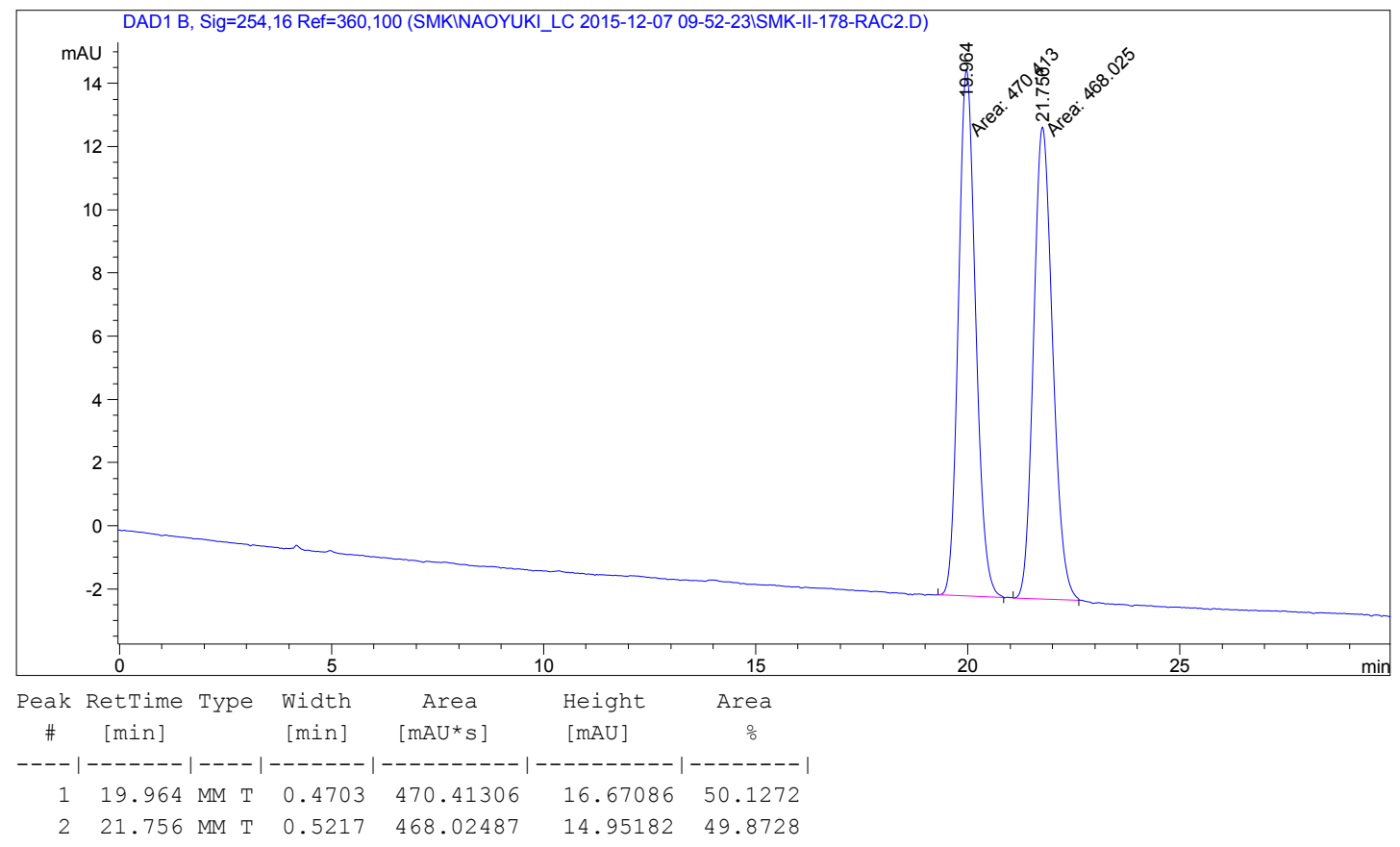

L-6n: $99 \%$ ee

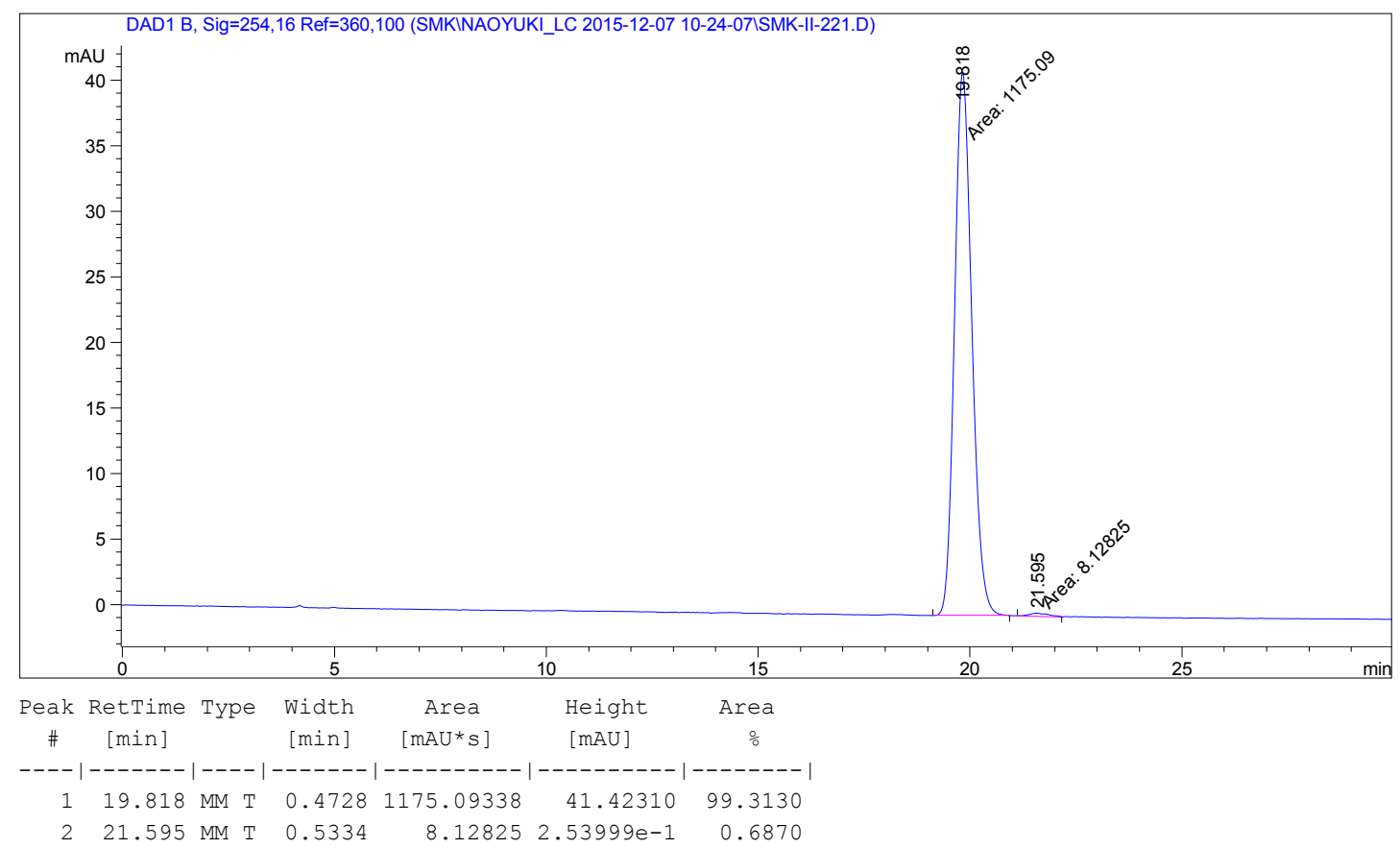


$\mathrm{MeS} \int_{\mathrm{NHPh}}^{\mathrm{CO}_{2} \mathrm{Me}}$

60

HPLC analysis (OJ-H, 10\% IPA-hexanes, $1.0 \mathrm{~mL} / \mathrm{min}, 254 \mathrm{~nm})$ indicated $80 \%$ ee: tR (minor) $=$ $20.3 \mathrm{~min}, \mathrm{tR}$ (major) $=29.6 \mathrm{~min}$.

\section{DL-6o}

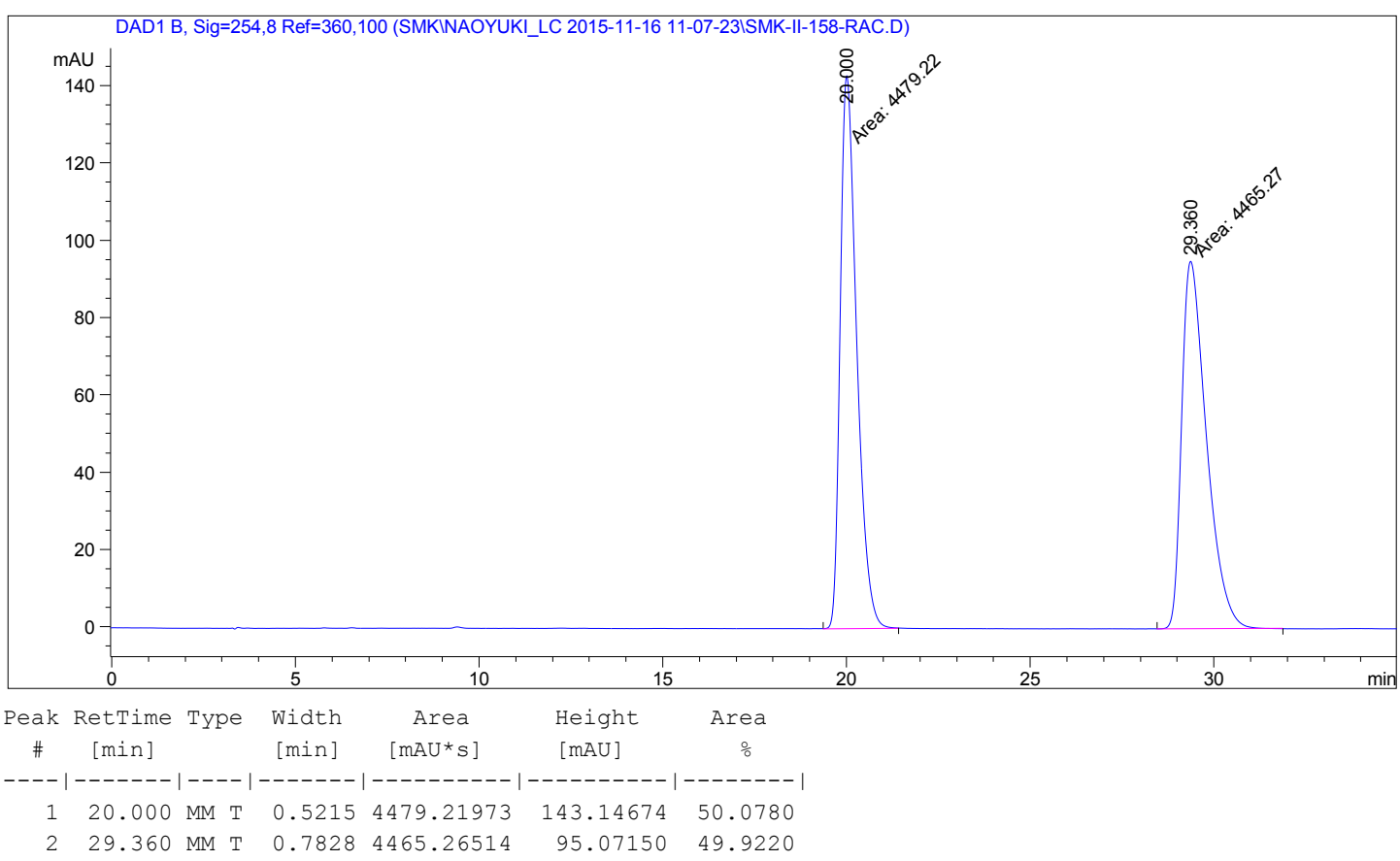

L-6o: $80 \%$ ee

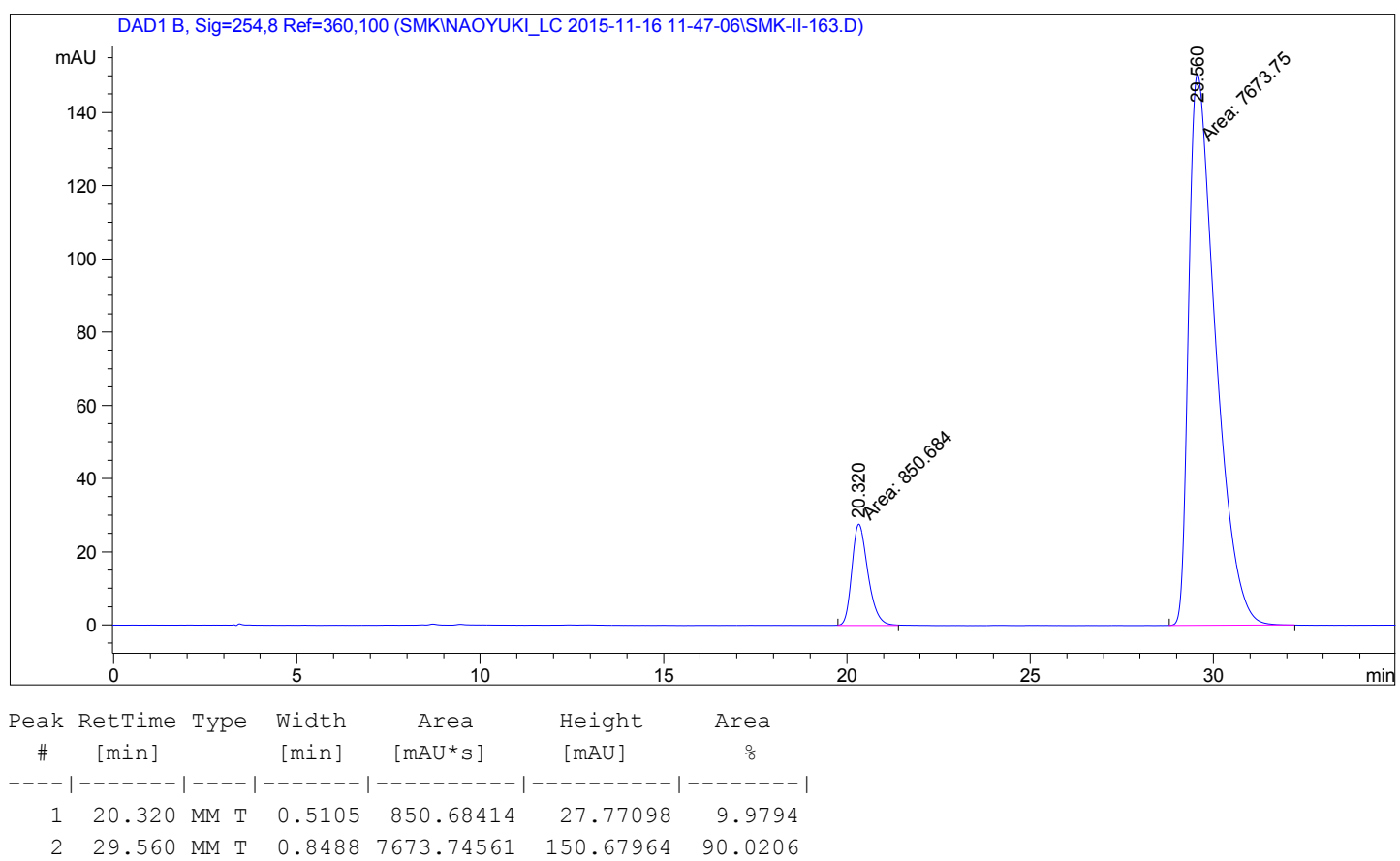




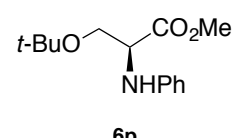

HPLC analysis (OJ-H, 10\% IPA-hexanes, $1.0 \mathrm{~mL} / \mathrm{min}, 254 \mathrm{~nm})$ indicated $71 \%$ ee: tR (minor) $=$ $7.1 \mathrm{~min}, \mathrm{tR}$ (major) $=10.9 \mathrm{~min}$.

\section{DL-6p}

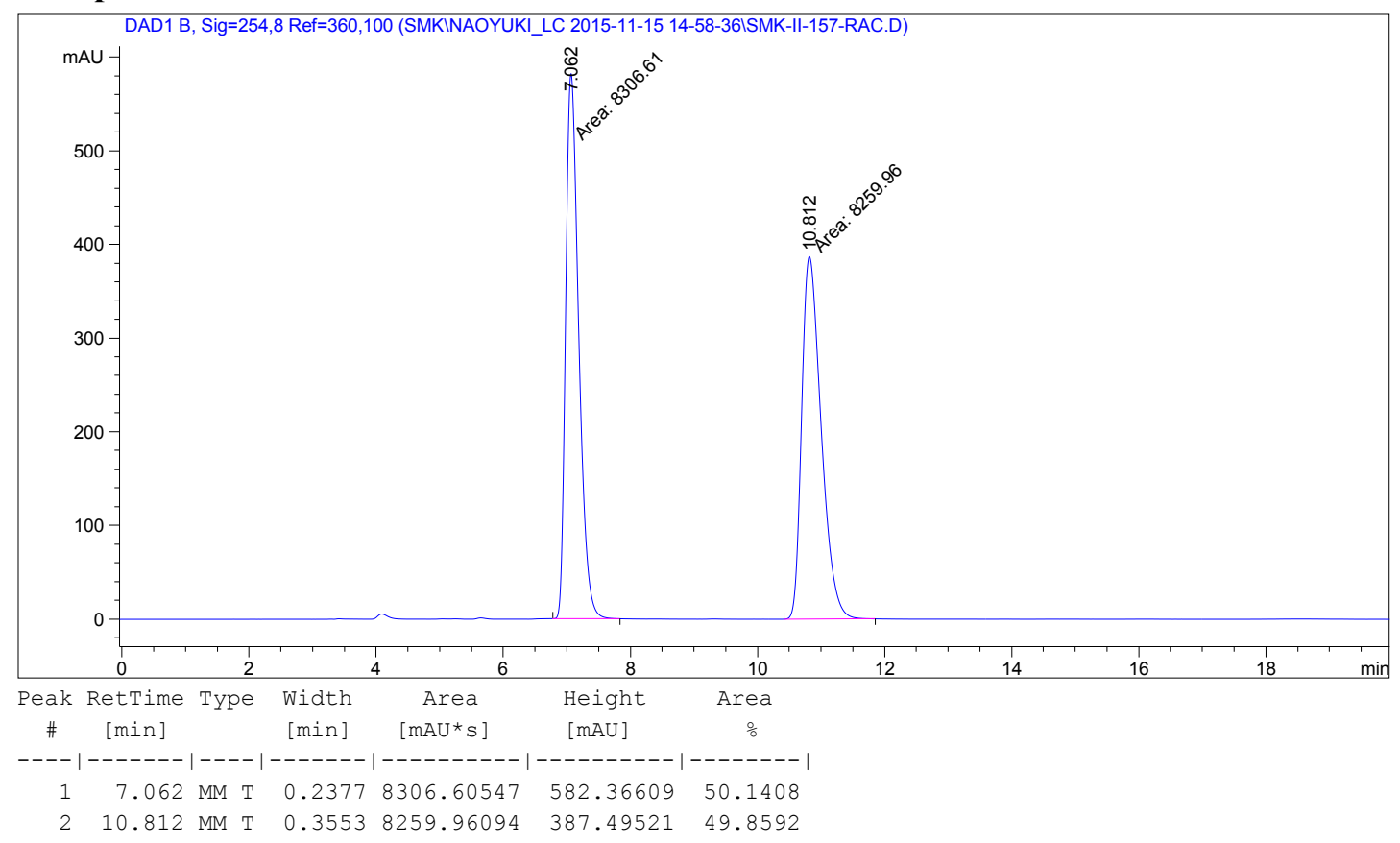

L-6p: $71 \%$ ee

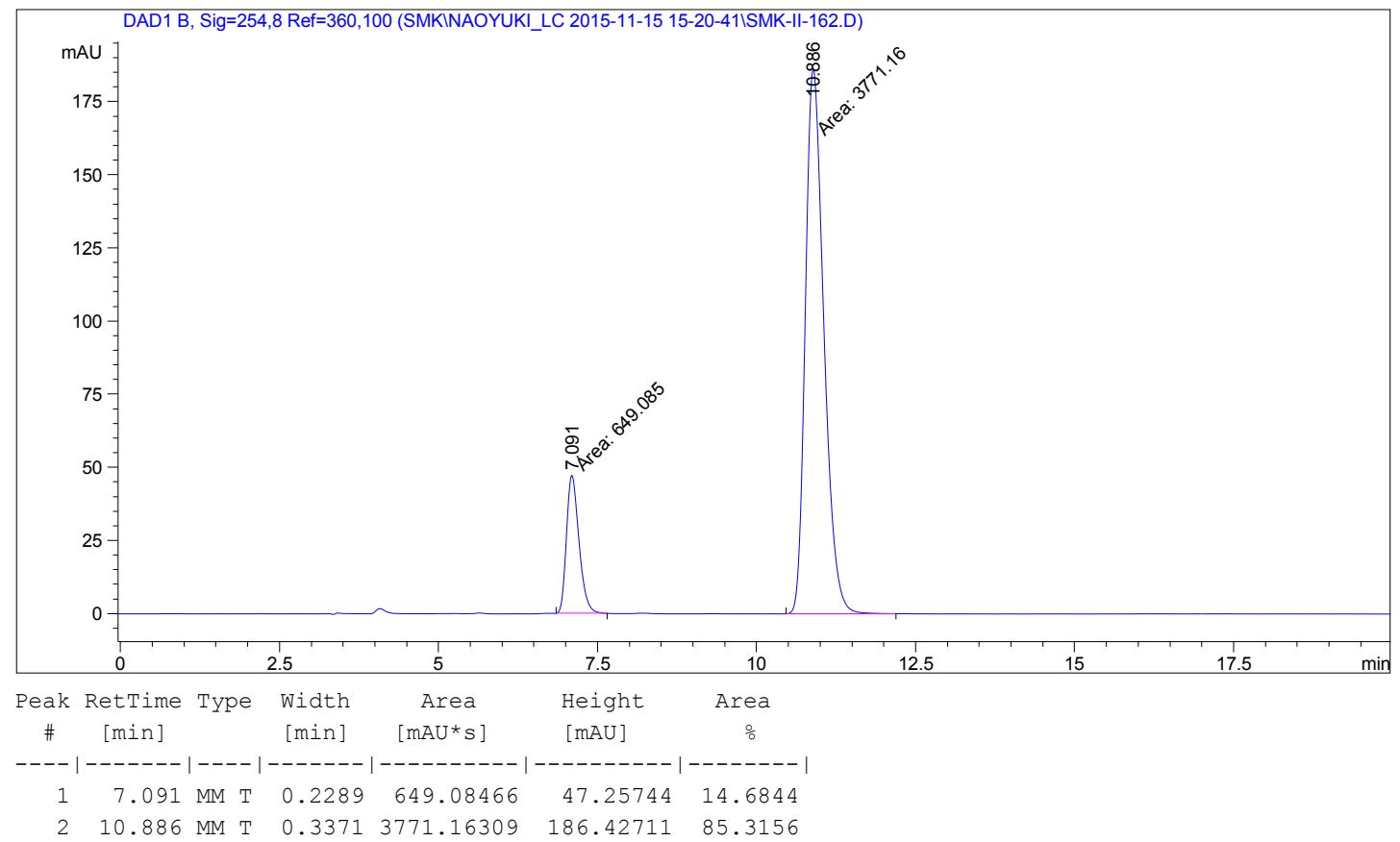


$\prod_{\mathrm{O}}^{\mathrm{MeO}} \mathrm{NHh}_{\mathrm{CO}_{2} \mathrm{Me}}^{\mathrm{C}_{\mathrm{H} h}}$

$6 q$

HPLC analysis (OJ-H, 10\% IPA-hexanes, $1.0 \mathrm{~mL} / \mathrm{min}, 254 \mathrm{~nm}$ ) indicated $74 \%$ ee: $\mathrm{tR}$ (minor) = $34.0 \mathrm{~min}$, tR (major) $=58.1 \mathrm{~min}$.

\section{DL-6q}

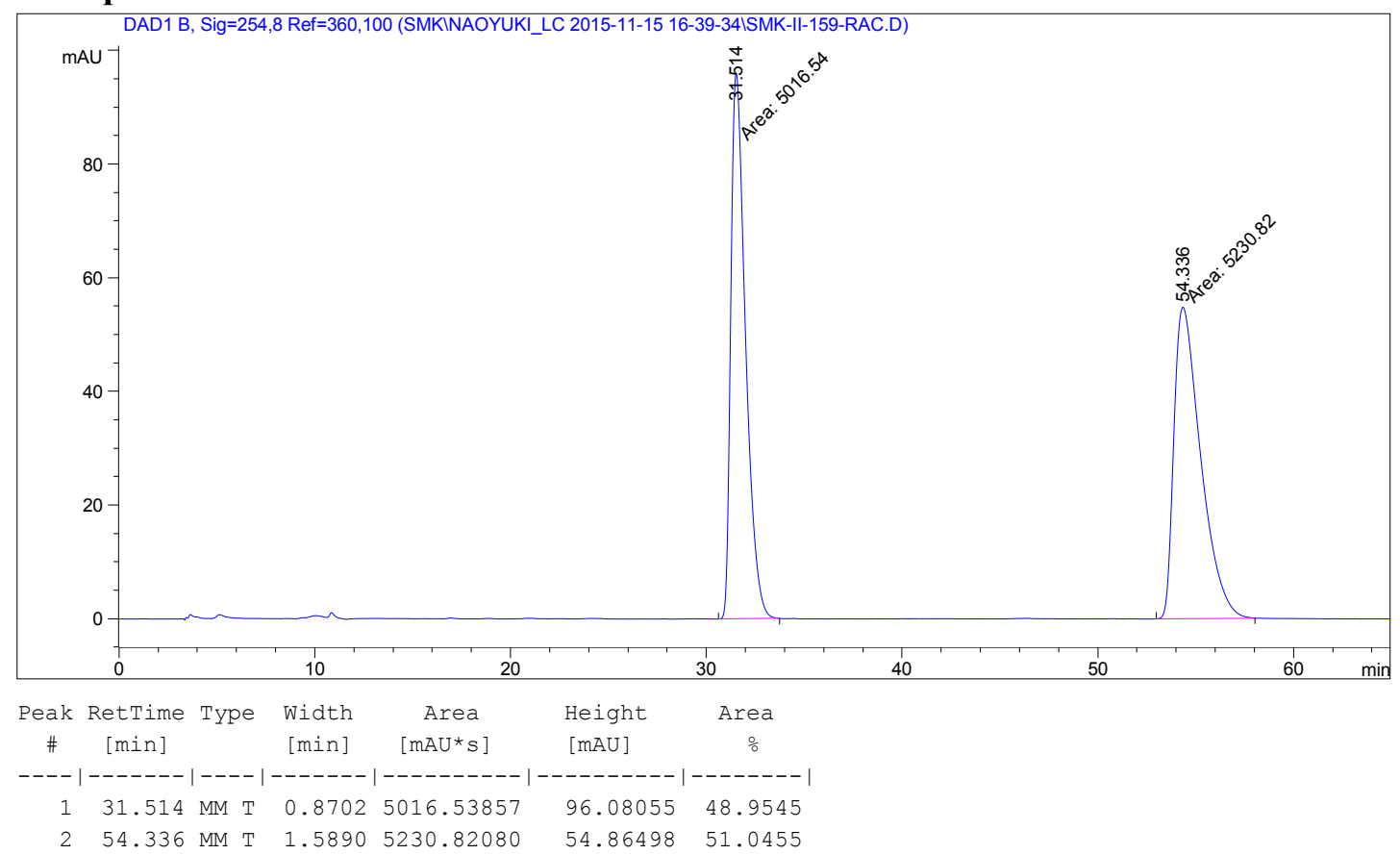

L-6q: $74 \%$ ee

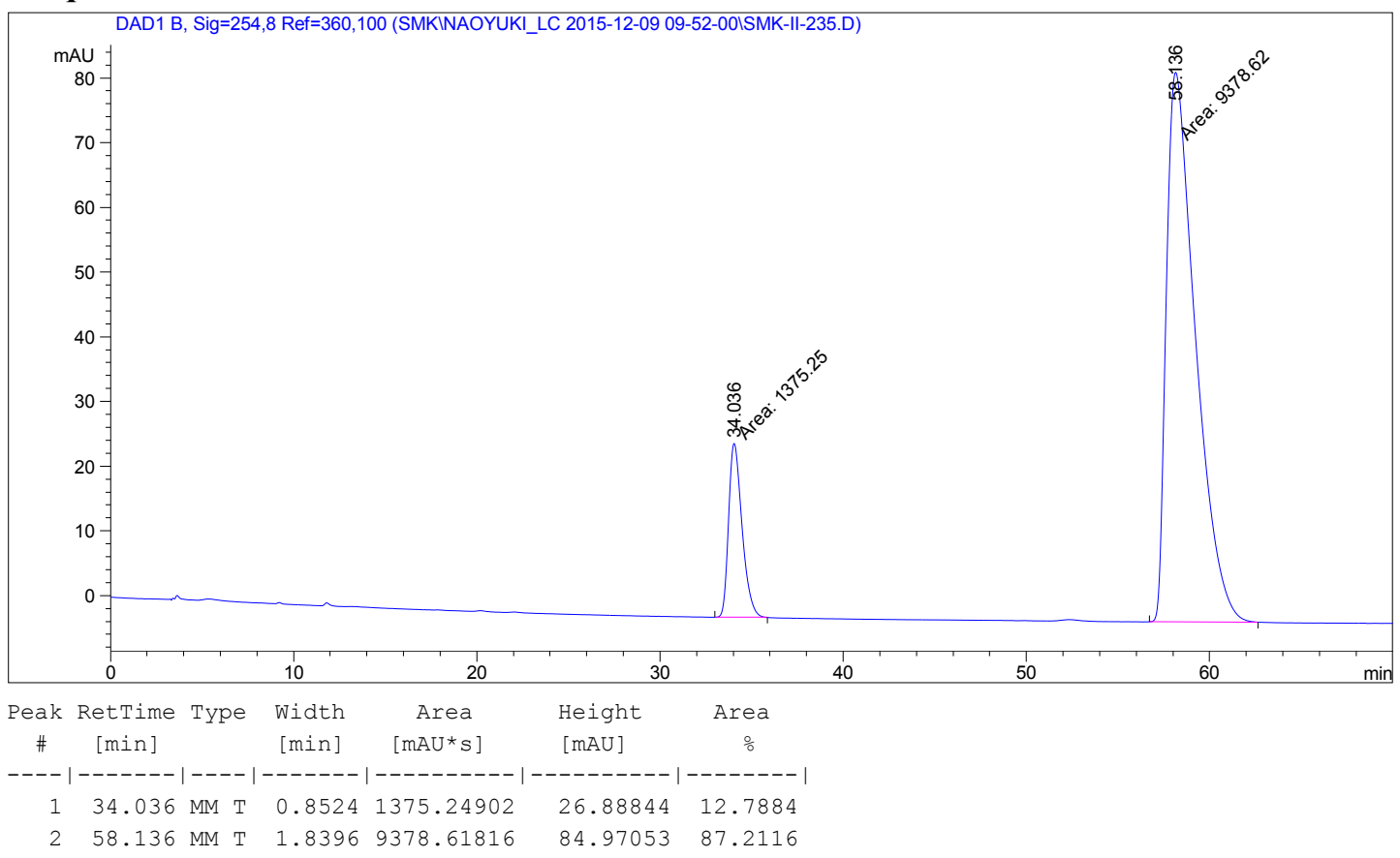


$\prod_{\mathrm{O} H \mathrm{Ph}}^{t-\mathrm{BuO}} \mathrm{CO}_{2} \mathrm{t}-\mathrm{Bu}$

$6 r$

HPLC analysis (OJ-H, 10\% IPA-hexanes, $1.0 \mathrm{~mL} / \mathrm{min}, 254 \mathrm{~nm})$ indicated $80 \%$ ee: tR (minor) $=$ $4.7 \mathrm{~min}, \mathrm{tR}$ (major) $=6.0 \mathrm{~min}$.

\section{DL-6r}

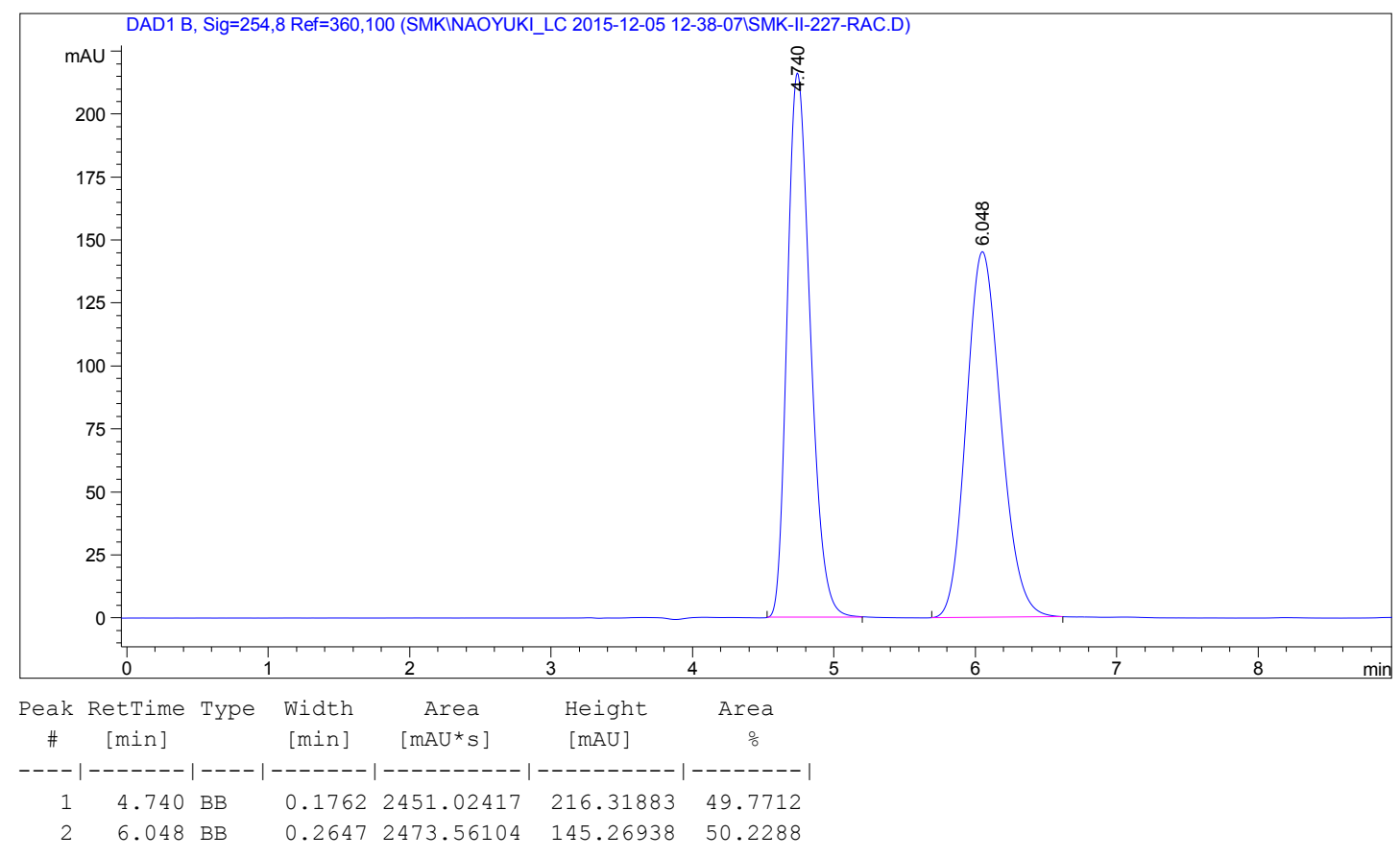

L-6r: $80 \%$ ee

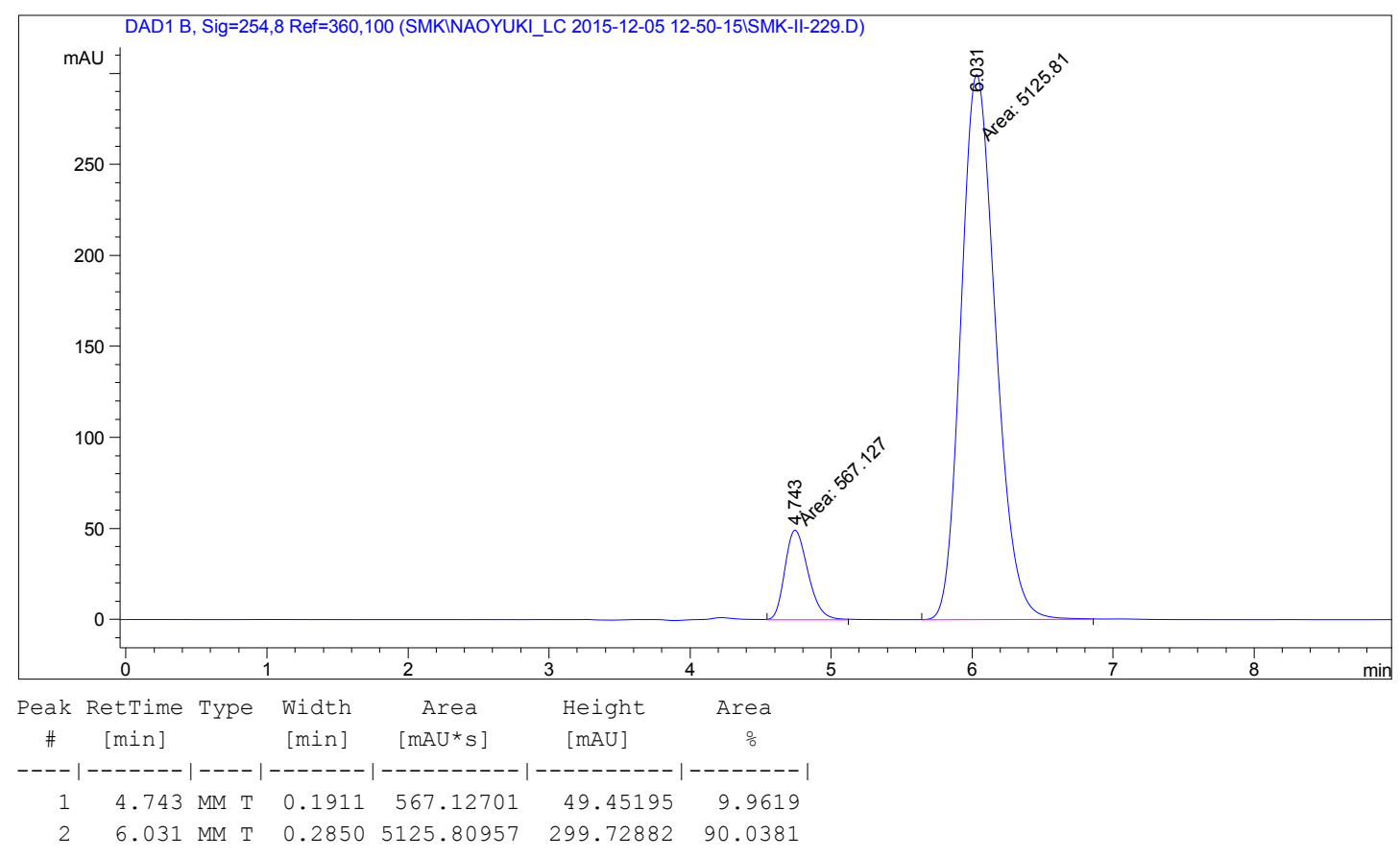




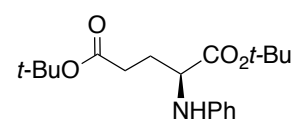

6s

HPLC analysis (OJ-H, 5\% IPA-hexanes, $0.8 \mathrm{~mL} / \mathrm{min}, 254 \mathrm{~nm})$ indicated $97 \%$ ee: tR (minor) $=$ $6.4 \mathrm{~min}, \mathrm{tR}$ (major) $=7.8 \mathrm{~min}$.

\section{DL-6s}

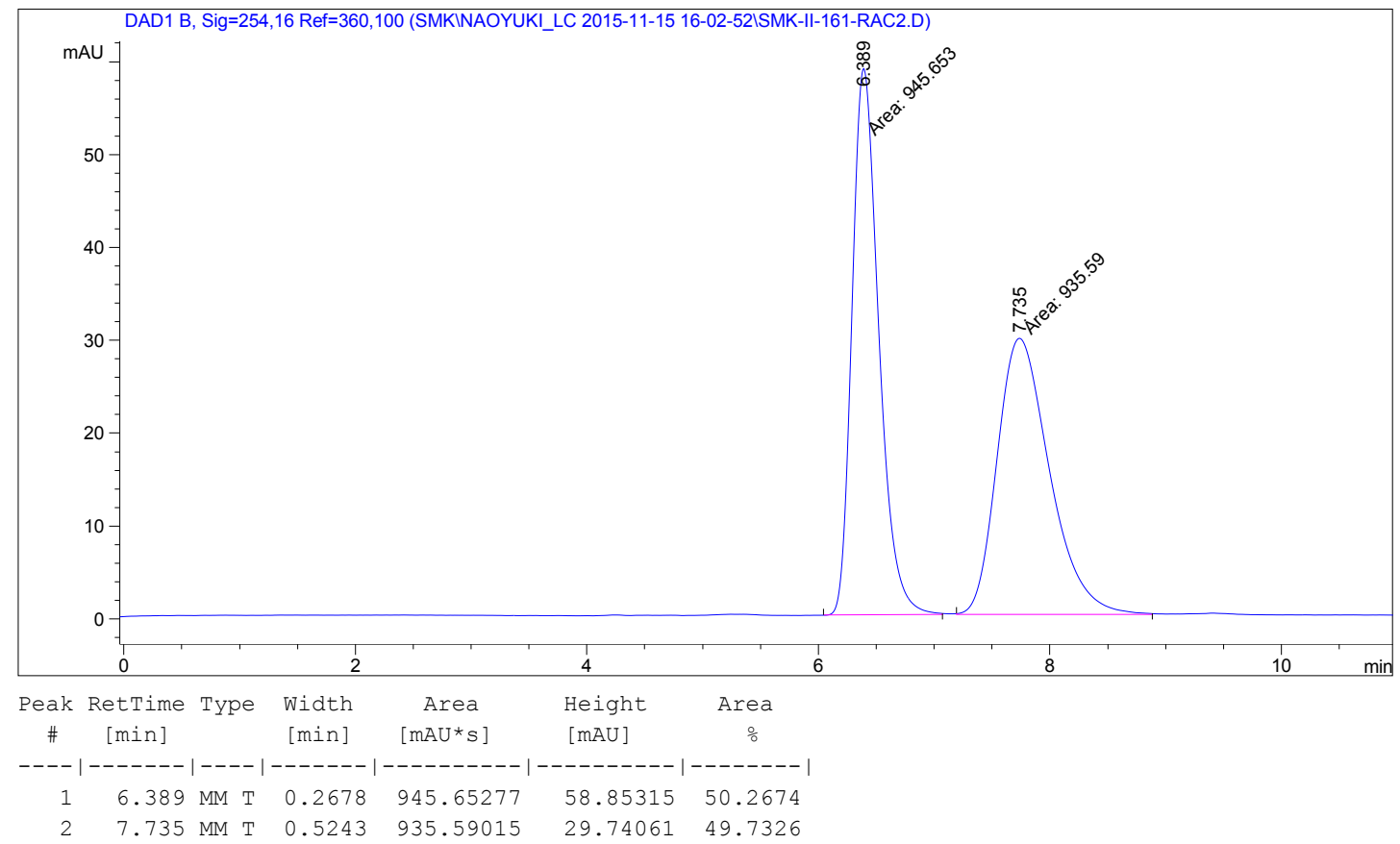

L-6s: $97 \%$ ee

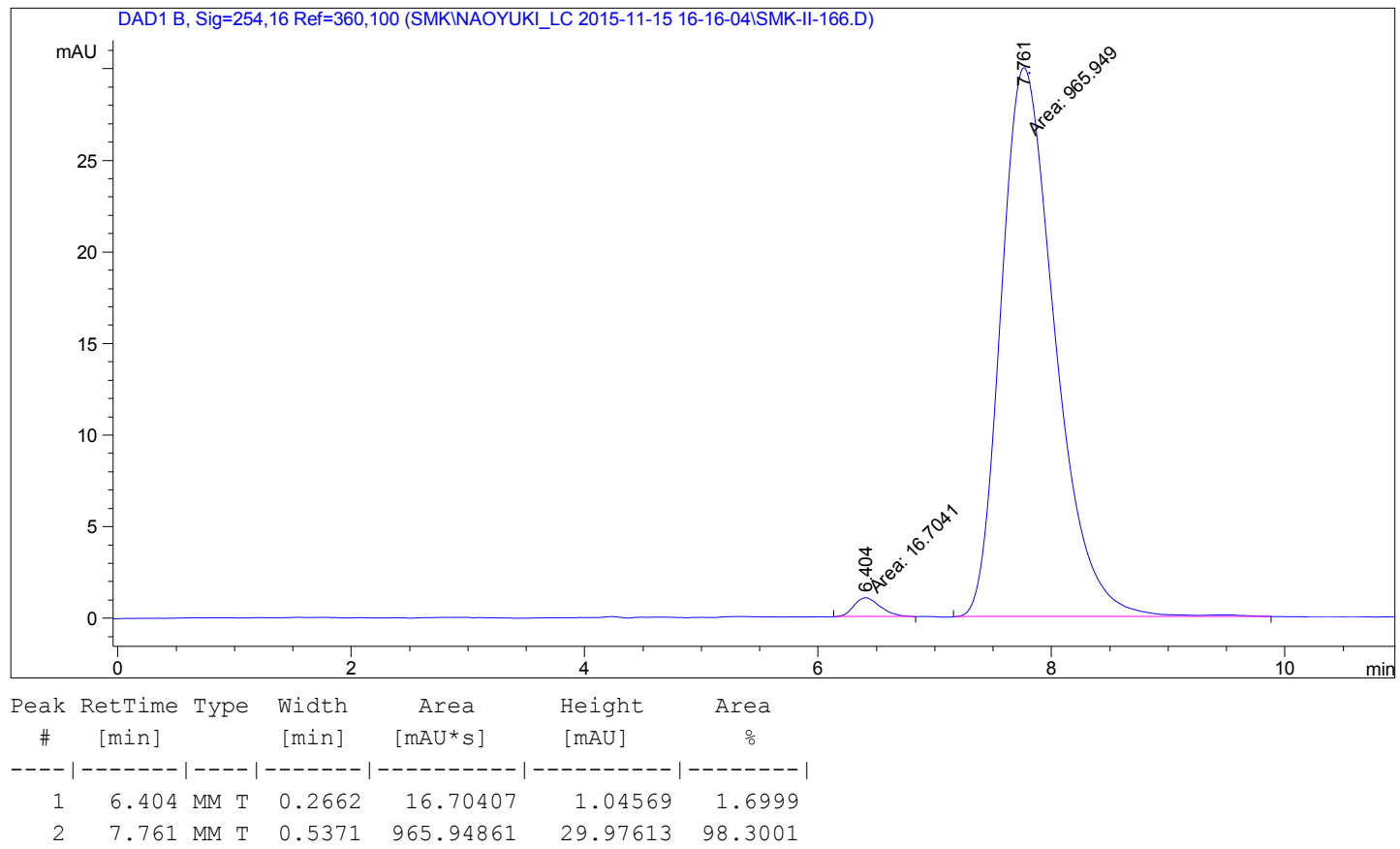




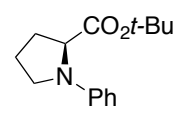

$6 t$

HPLC analysis (OJ-H, 5\% IPA-hexanes, $0.8 \mathrm{~mL} / \mathrm{min}, 254 \mathrm{~nm})$ indicated $44 \%$ ee: tR (minor) $=$ $7.0 \mathrm{~min}, \mathrm{tR}$ (major) $=10.3 \mathrm{~min}$.

\section{DL-6t}

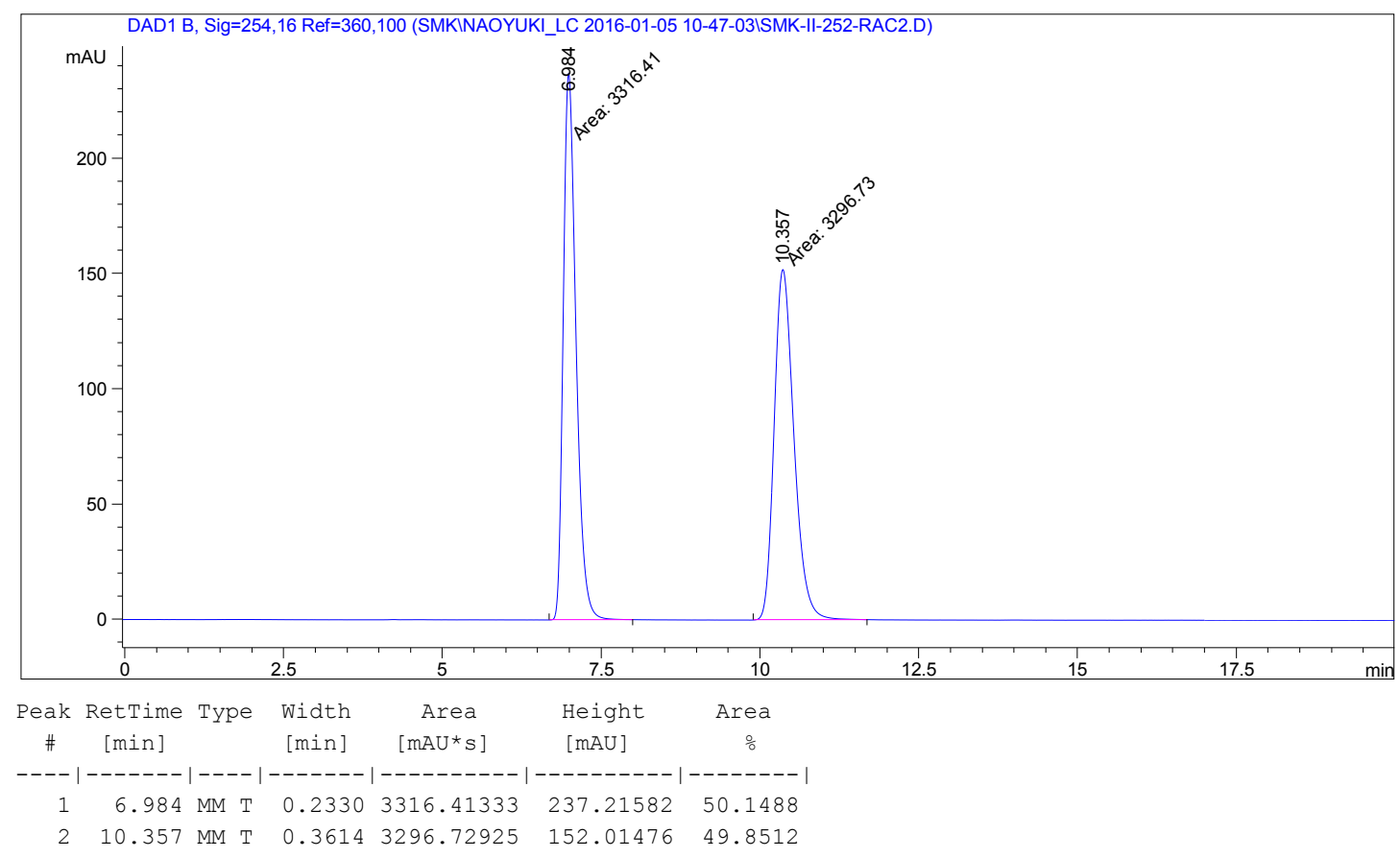

L-6t: $44 \%$ ee

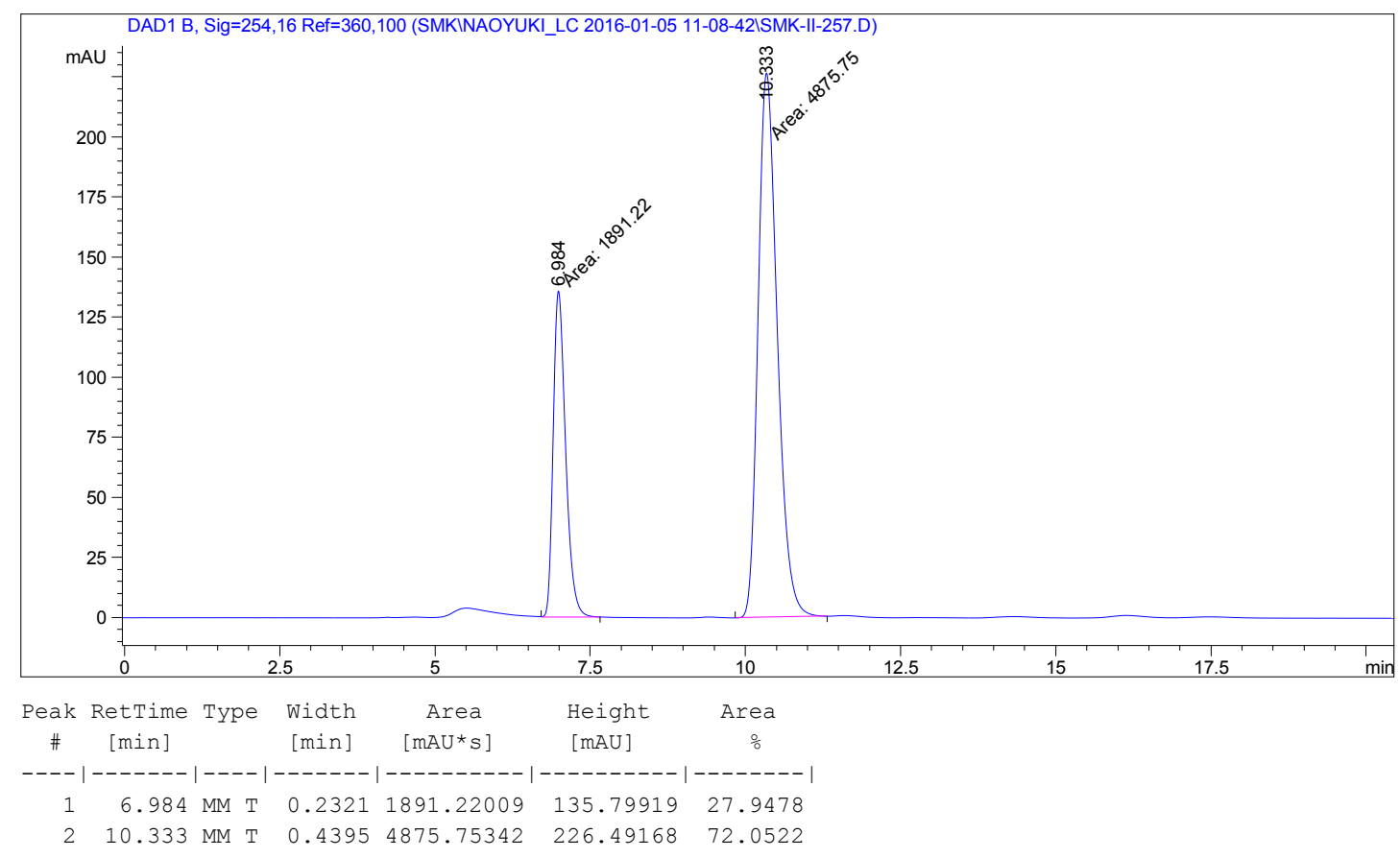


L-6t: $97 \%$ ee

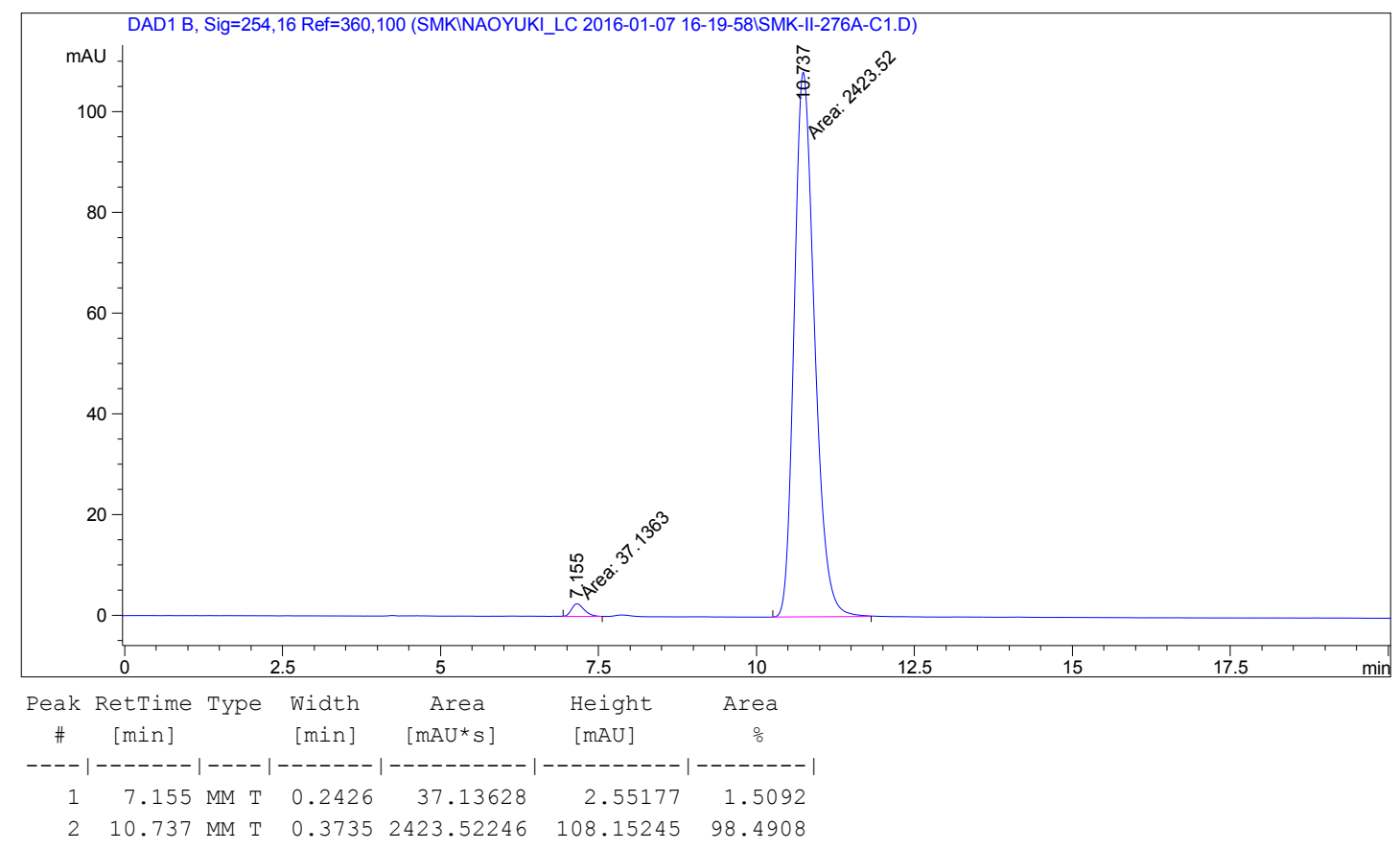




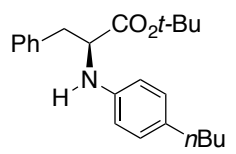

$8 a$

HPLC analysis (OJ-H, 5\% IPA-hexanes, $0.8 \mathrm{~mL} / \mathrm{min}, 254 \mathrm{~nm})$ indicated $90 \%$ ee: tR (minor) $=$ 8.4 min, $\mathrm{tR}$ (major) $=10.6 \mathrm{~min}$.

\section{DL-8a}

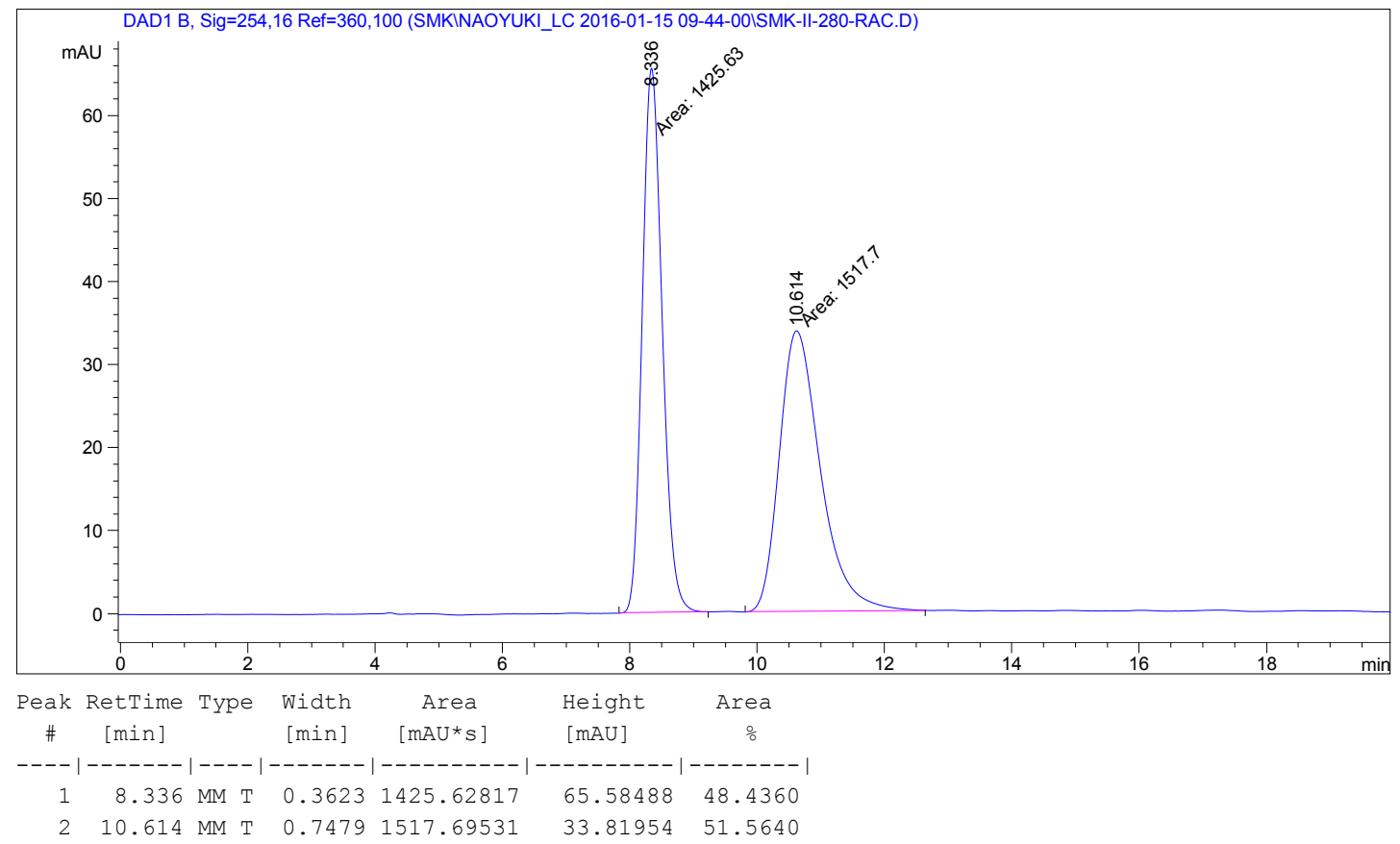

L-8a: $90 \%$ ee

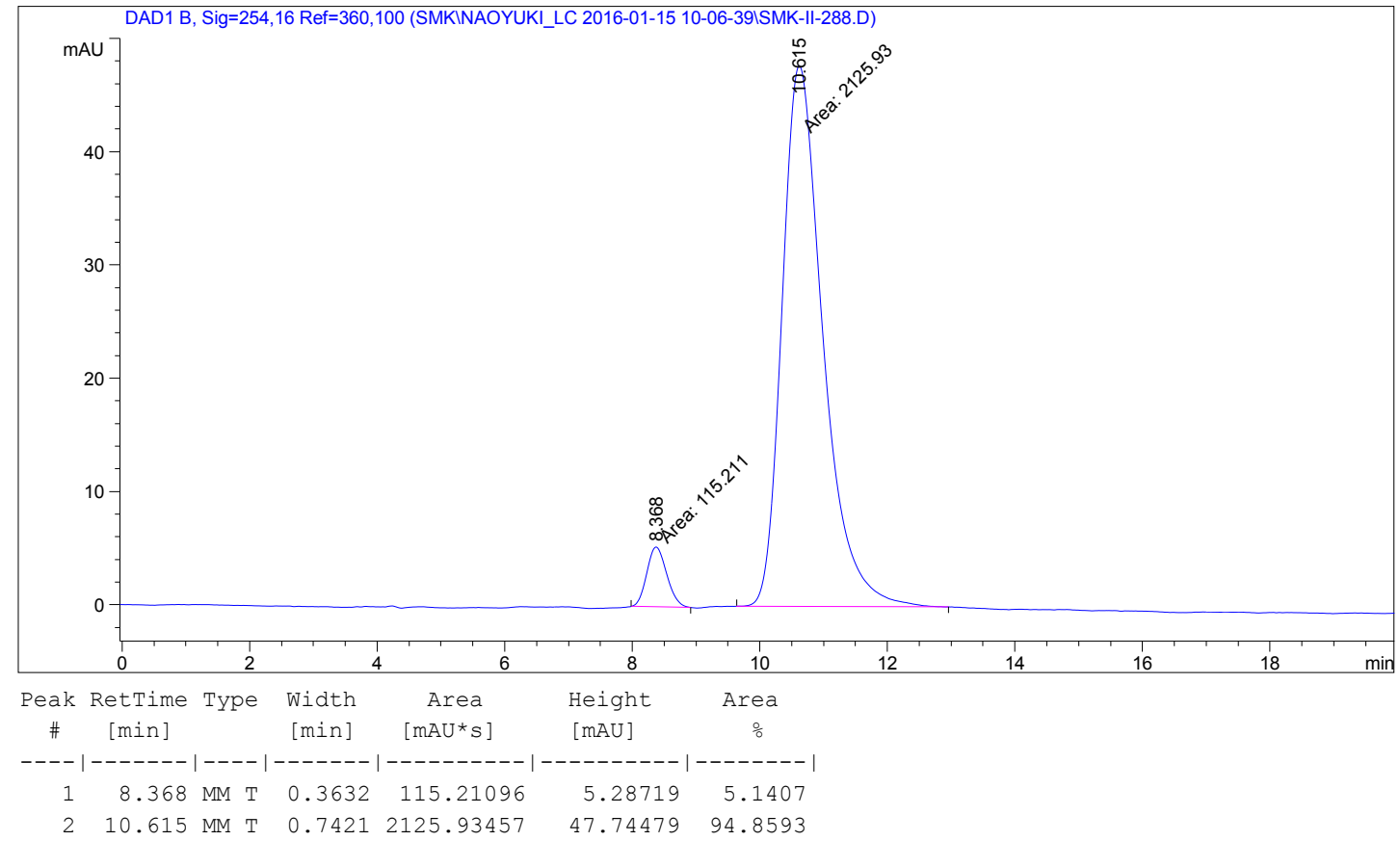




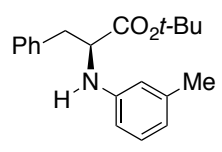

$8 \mathrm{~b}$

HPLC analysis (OJ-H, 2\% IPA-hexanes, $0.8 \mathrm{~mL} / \mathrm{min}, 254 \mathrm{~nm})$ indicated $87 \%$ ee: tR (minor) $=$ $16.1 \mathrm{~min}, \mathrm{tR}$ (major) $=20.3 \mathrm{~min}$.

\section{DL-8b}

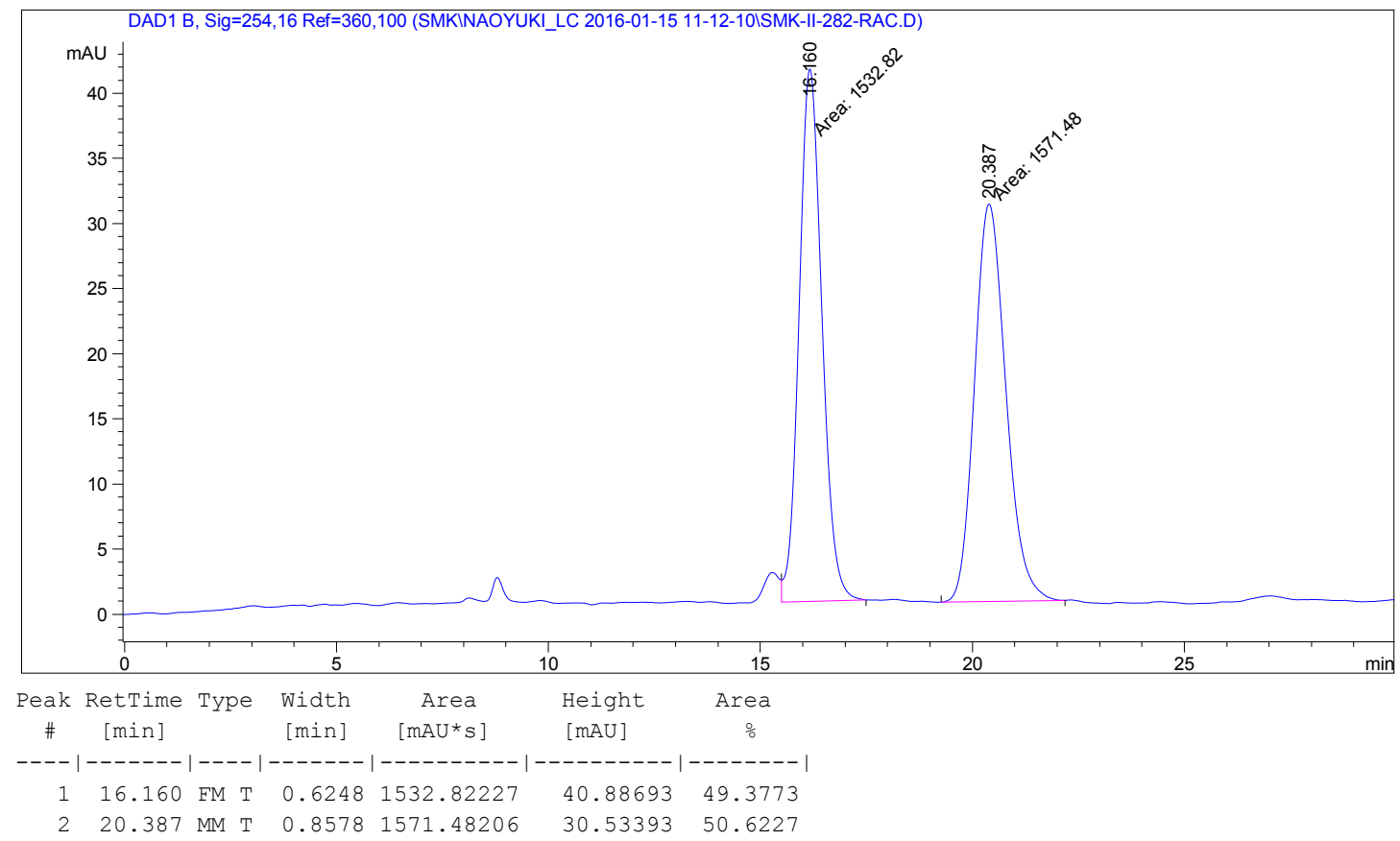

L-8b: $87 \%$ ee

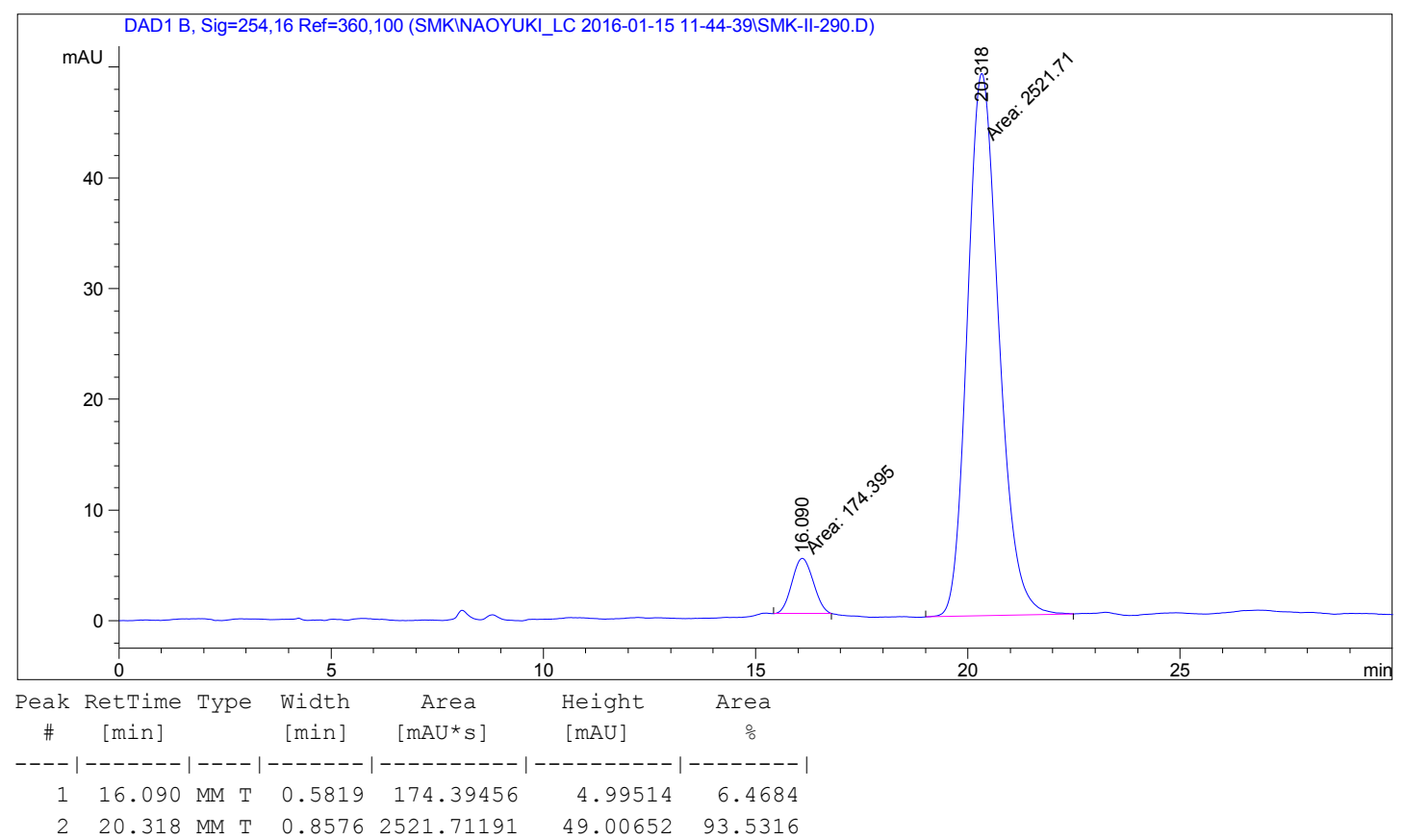




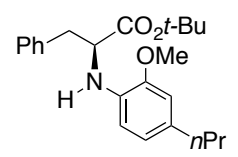

$8 c$

HPLC analysis (OJ-H, 2\% IPA-hexanes, $0.8 \mathrm{~mL} / \mathrm{min}, 254 \mathrm{~nm})$ indicated $88 \%$ ee: tR (minor) $=$ 8.9 min, $\mathrm{tR}($ major $)=10.8 \mathrm{~min}$.

\section{DL-8c}

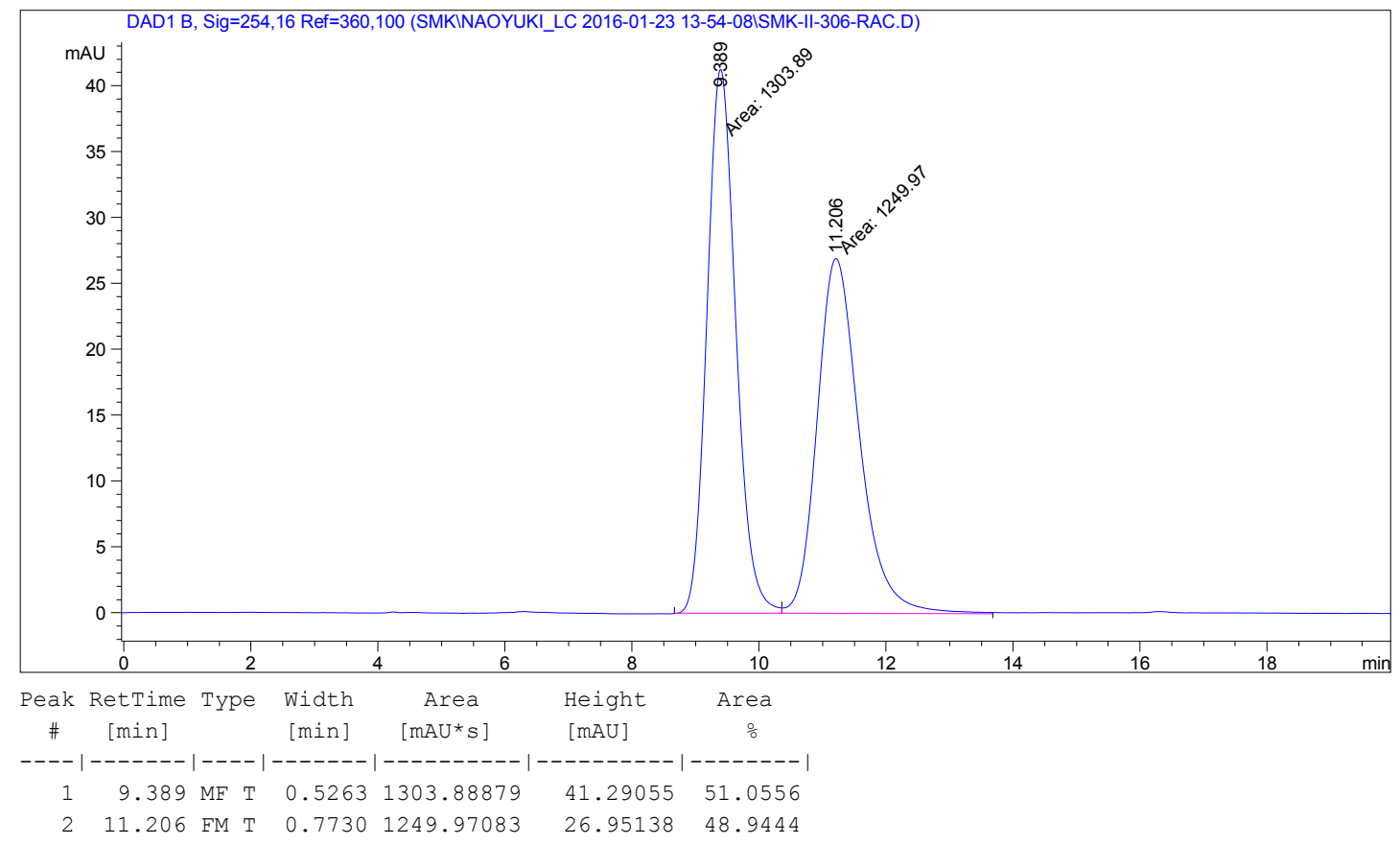

L-8c: $88 \%$ ee

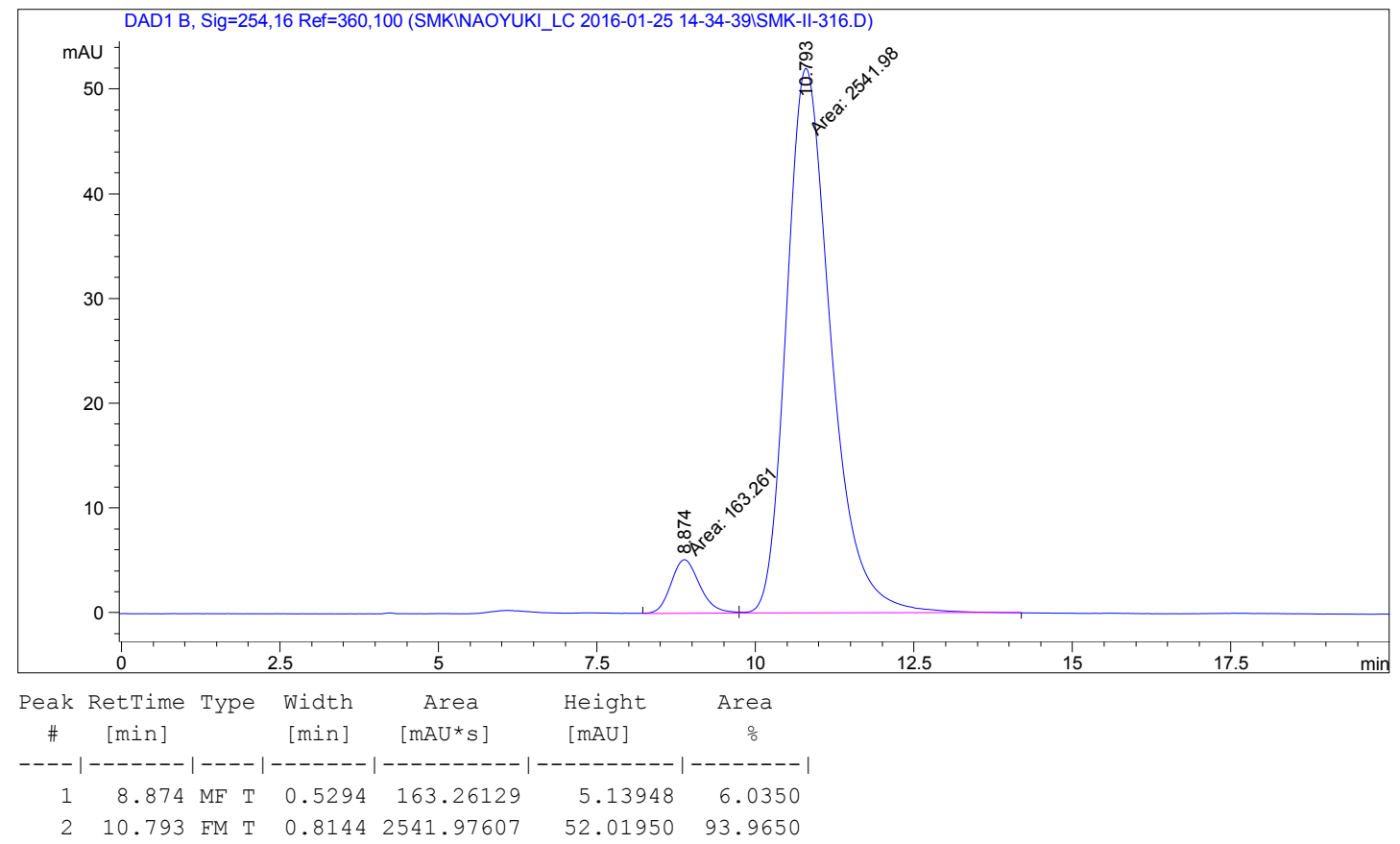




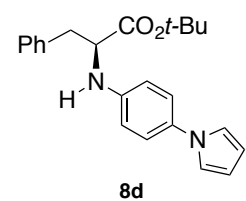

HPLC analysis (OJ-H, 10\% IPA-hexanes, $1.0 \mathrm{~mL} / \mathrm{min}, 254 \mathrm{~nm}$ ) indicated $91 \%$ ee: $\mathrm{tR}$ (major) = $38.3 \mathrm{~min}, \mathrm{tR}($ minor $)=50.5 \mathrm{~min}$.

\section{DL-8d}

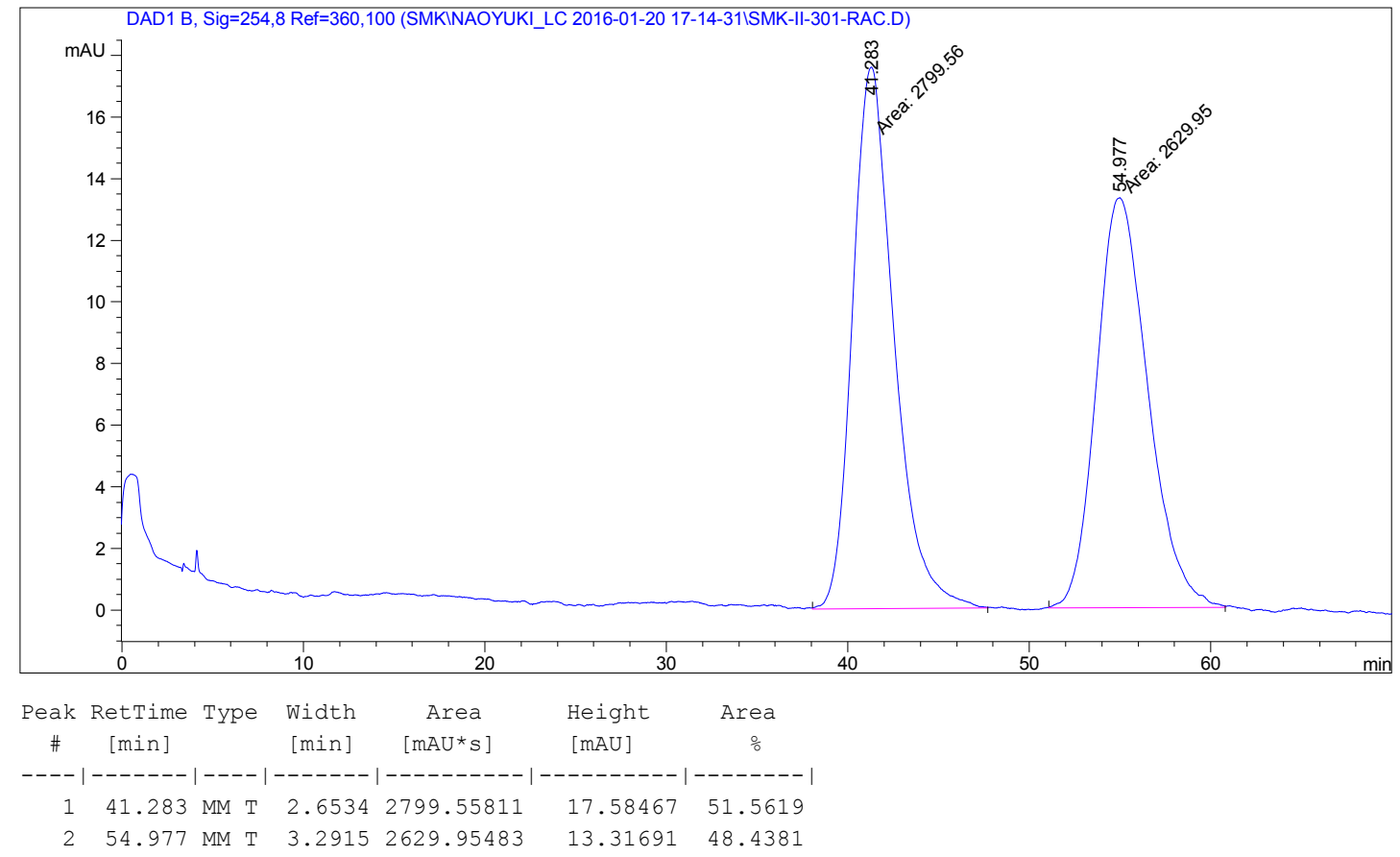

L-8d: $91 \%$ ee

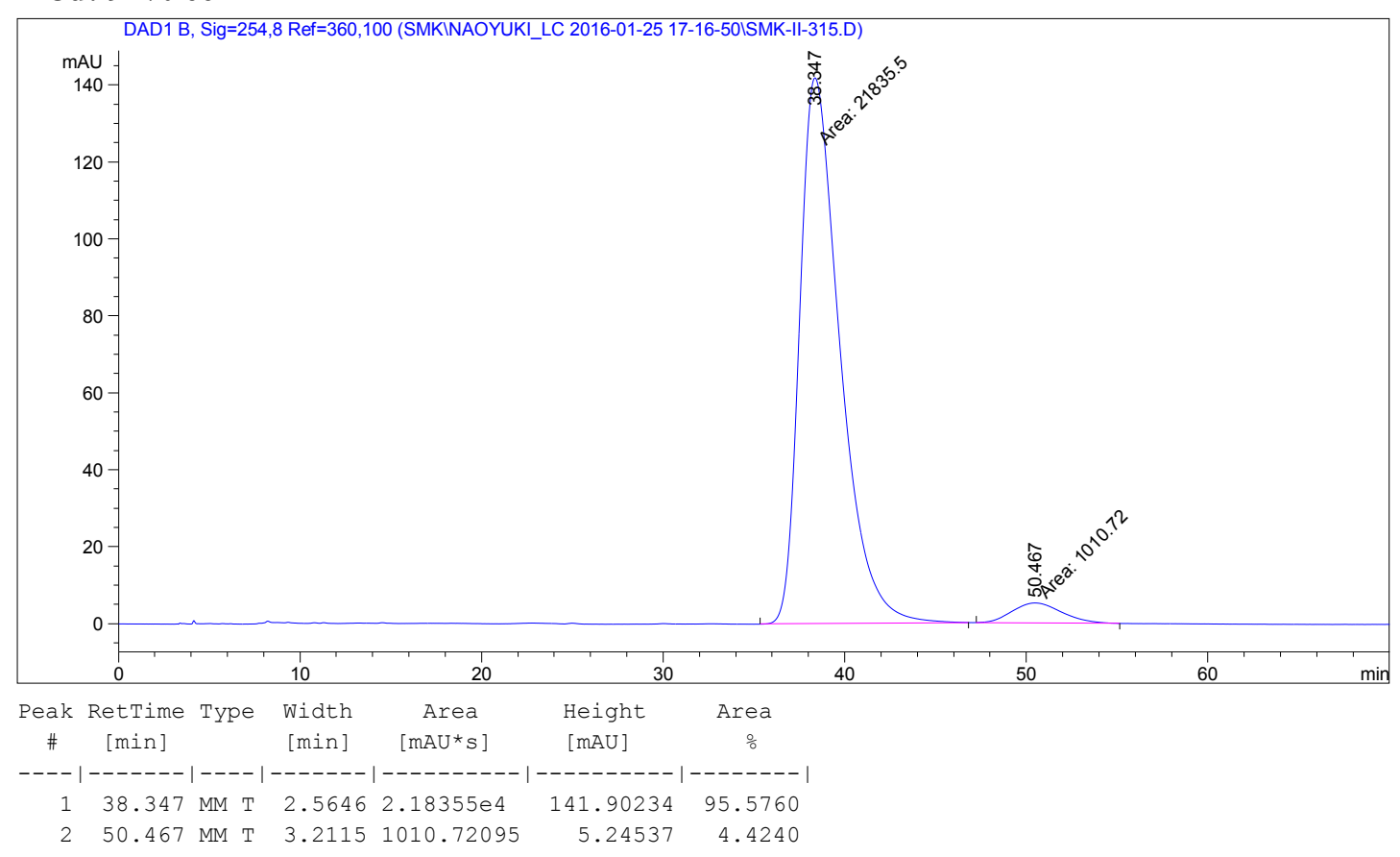




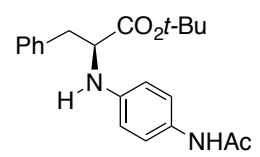

$8 \mathrm{e}$

HPLC analysis (AD-H, 10\% IPA-hexanes, $1.0 \mathrm{~mL} / \mathrm{min}, 254 \mathrm{~nm}$ ) indicated $86 \%$ ee: tR (minor) = $17.4 \mathrm{~min}, \mathrm{tR}$ (major) $=23.7 \mathrm{~min}$.

\section{DL-8e}

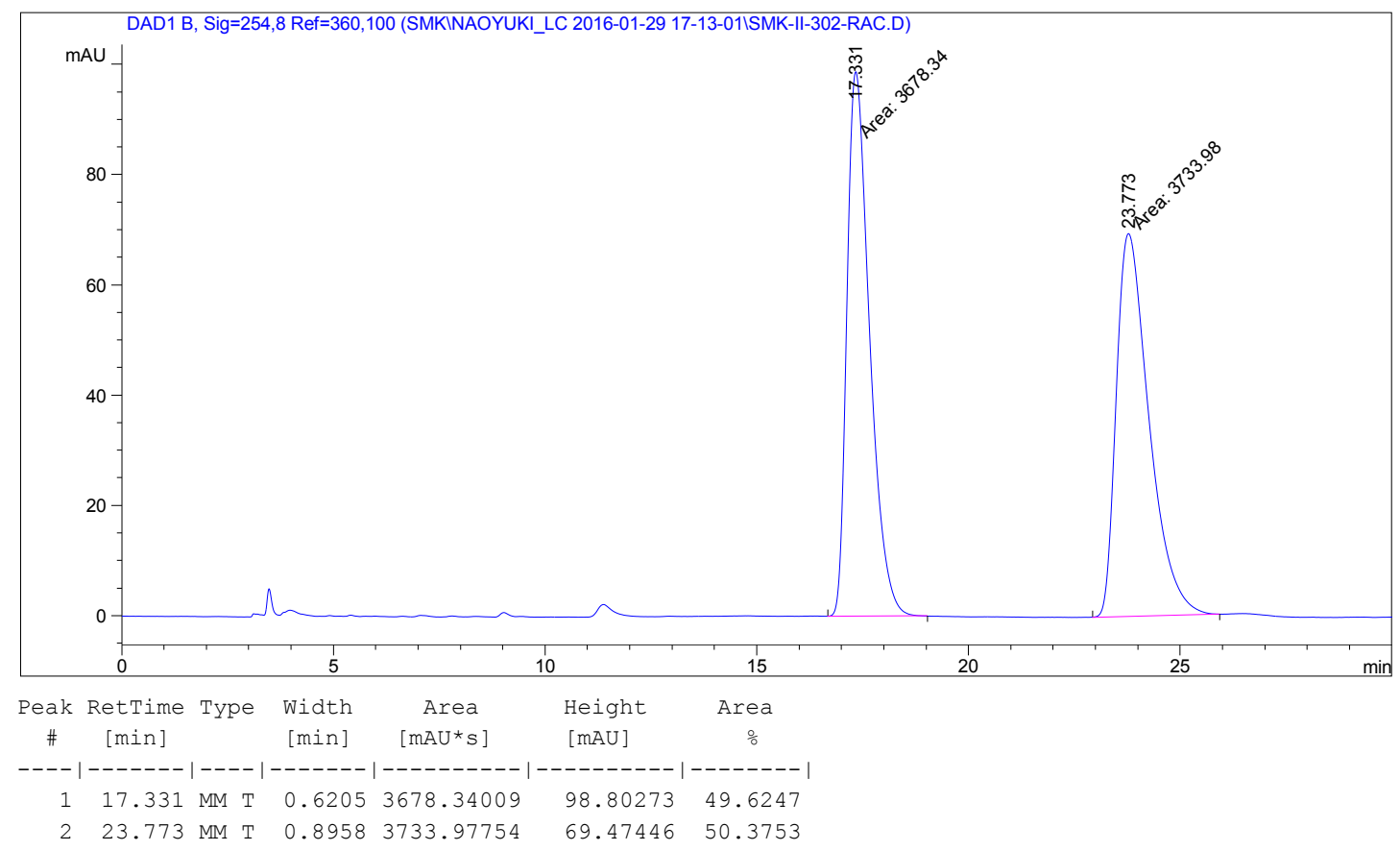

L-8e: $86 \%$ ee

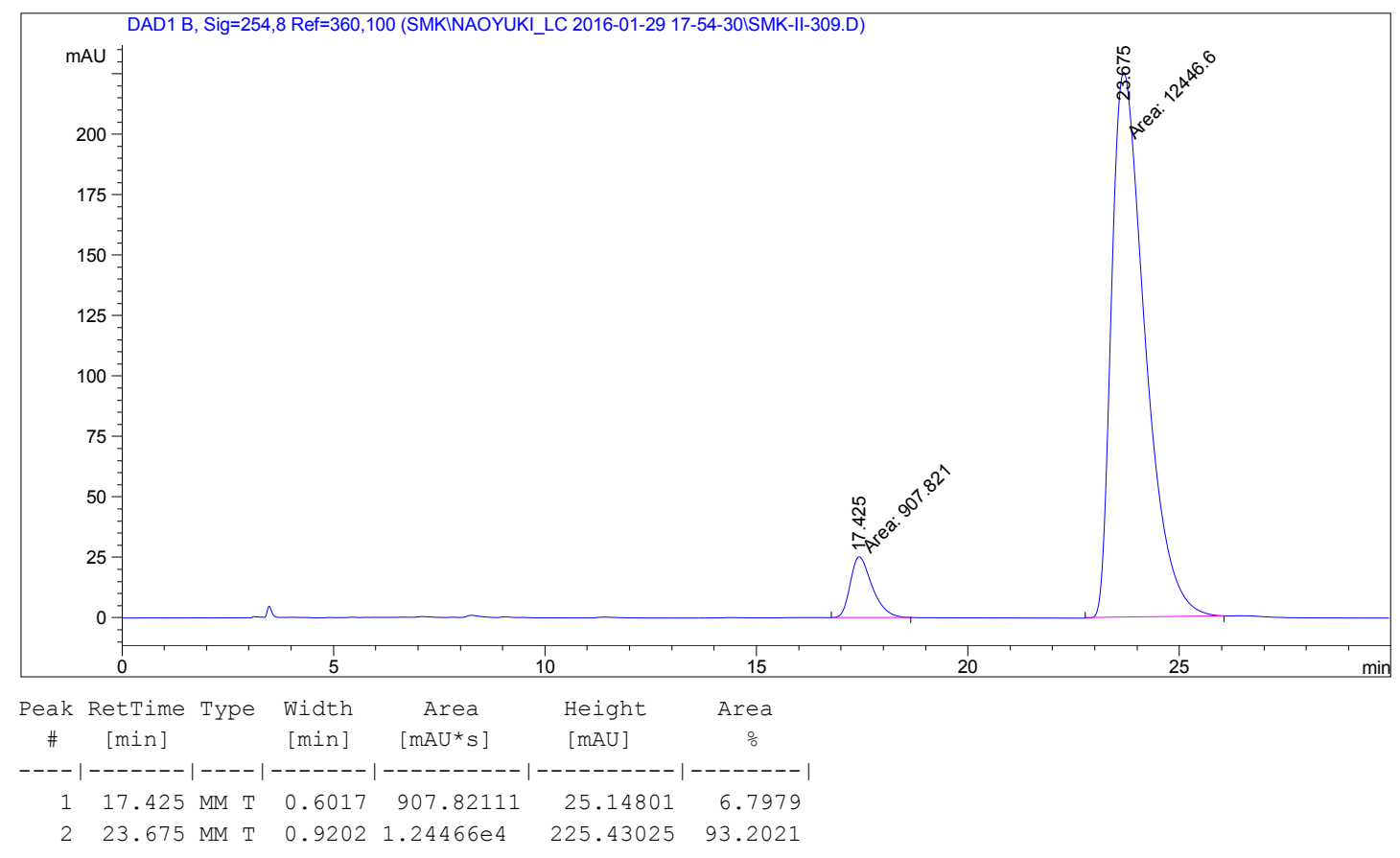




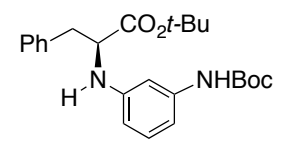

$8 f$

HPLC analysis (AD-H, 10\% IPA-hexanes, $0.8 \mathrm{~mL} / \mathrm{min}, 254 \mathrm{~nm}$ ) indicated $80 \%$ ee: $\mathrm{tR}$ (major) = $12.8 \mathrm{~min}, \mathrm{tR}($ minor $)=15.0 \mathrm{~min}$.

\section{DL-8f}

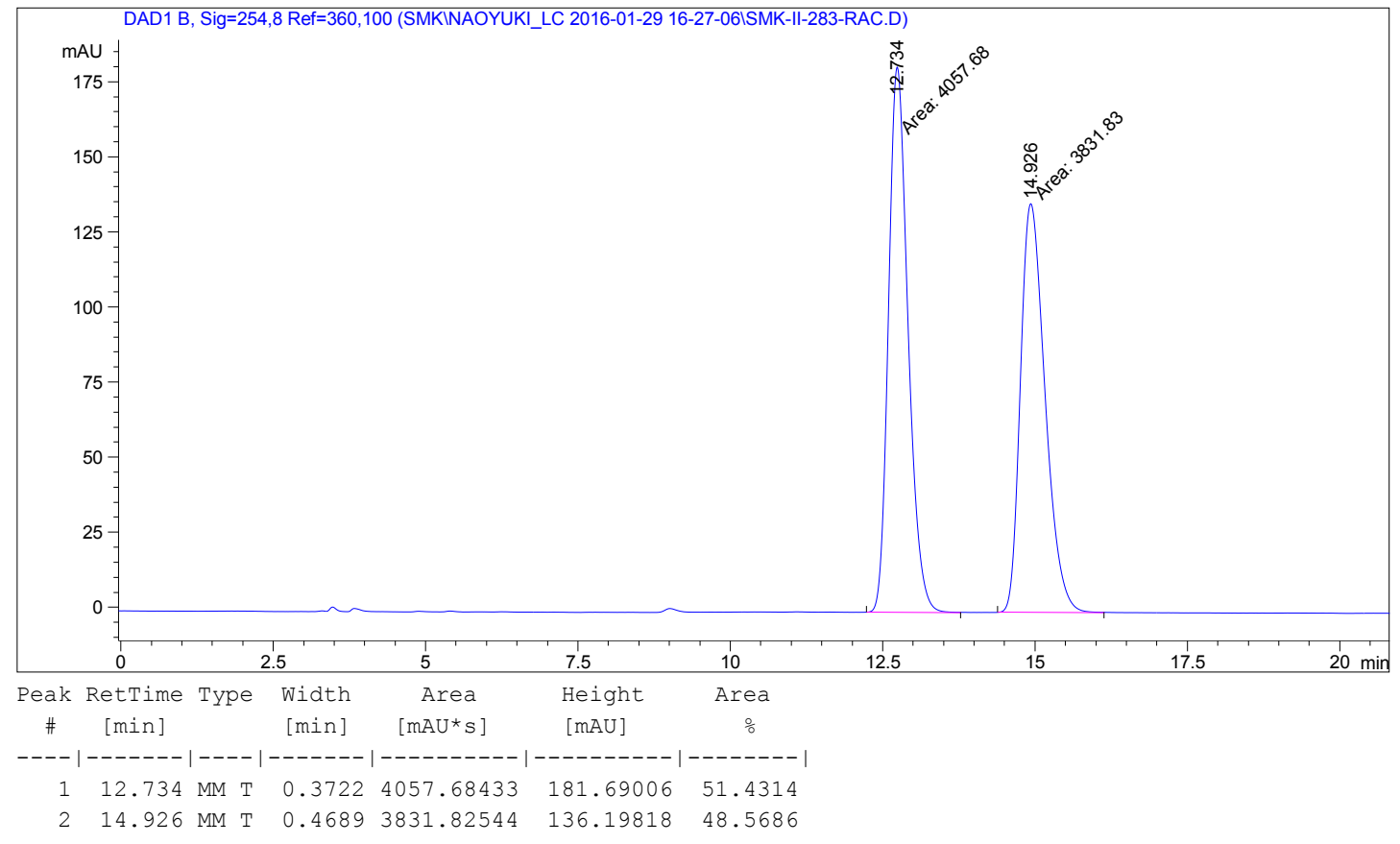

L-8f: $80 \%$ ee

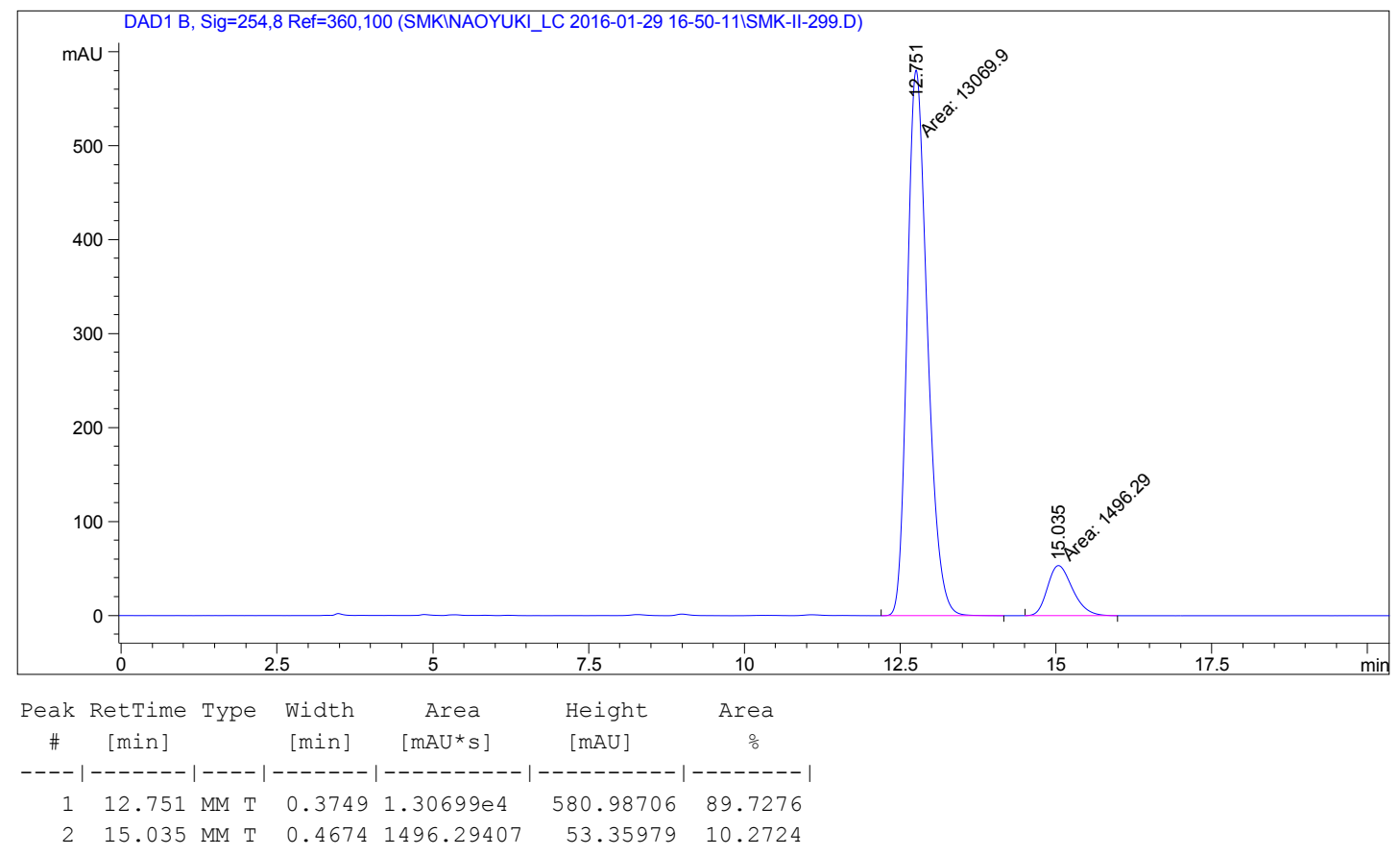




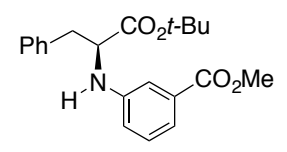

$8 g$

HPLC analysis (OJ-H, 10\% IPA-hexanes, $1.0 \mathrm{~mL} / \mathrm{min}, 254 \mathrm{~nm}$ ) indicated $82 \%$ ee: tR (major) = 11.9 min, $\mathrm{tR}($ minor $)=18.8 \mathrm{~min}$.

\section{DL-8g}

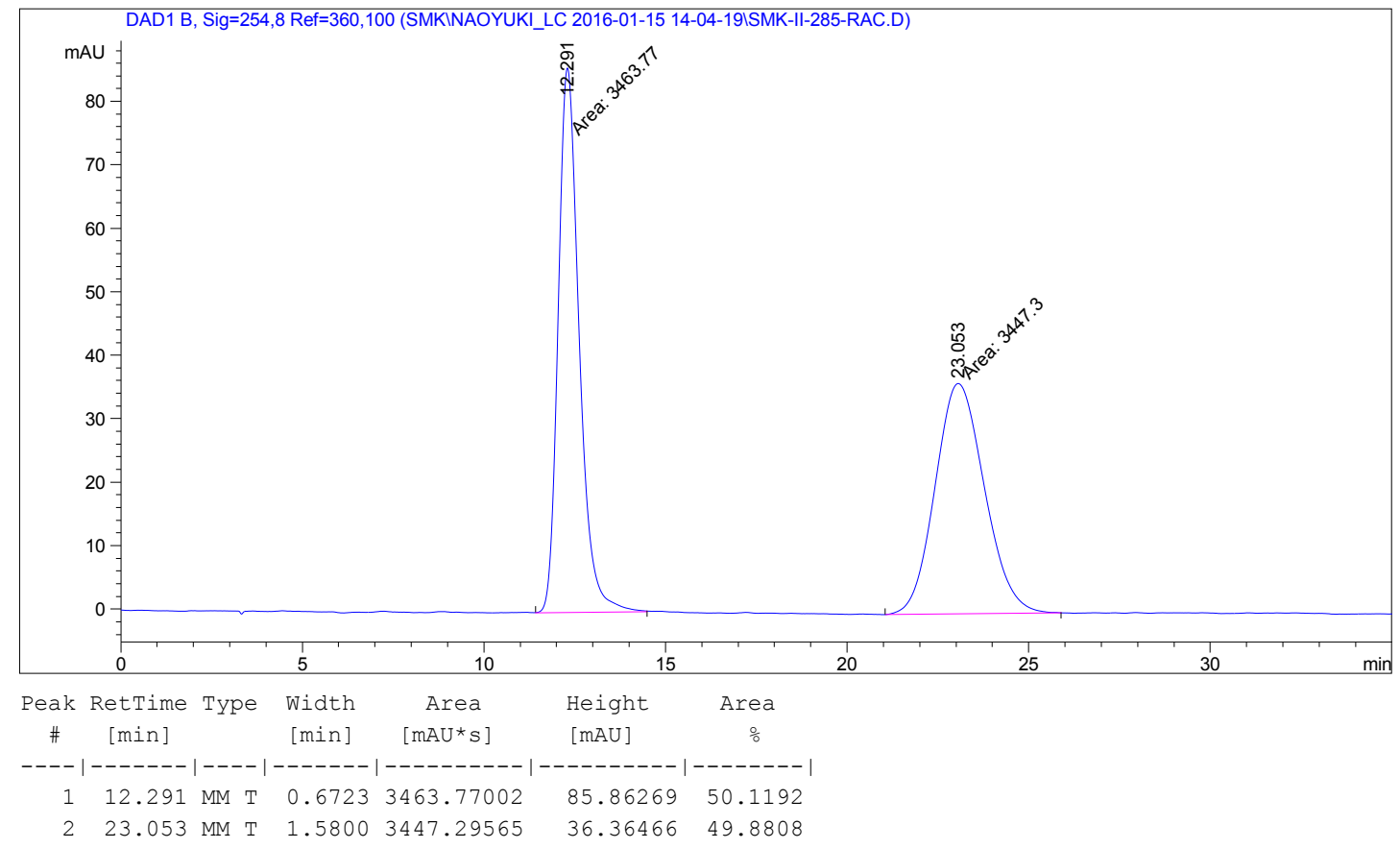

L-8g: $82 \%$ ee

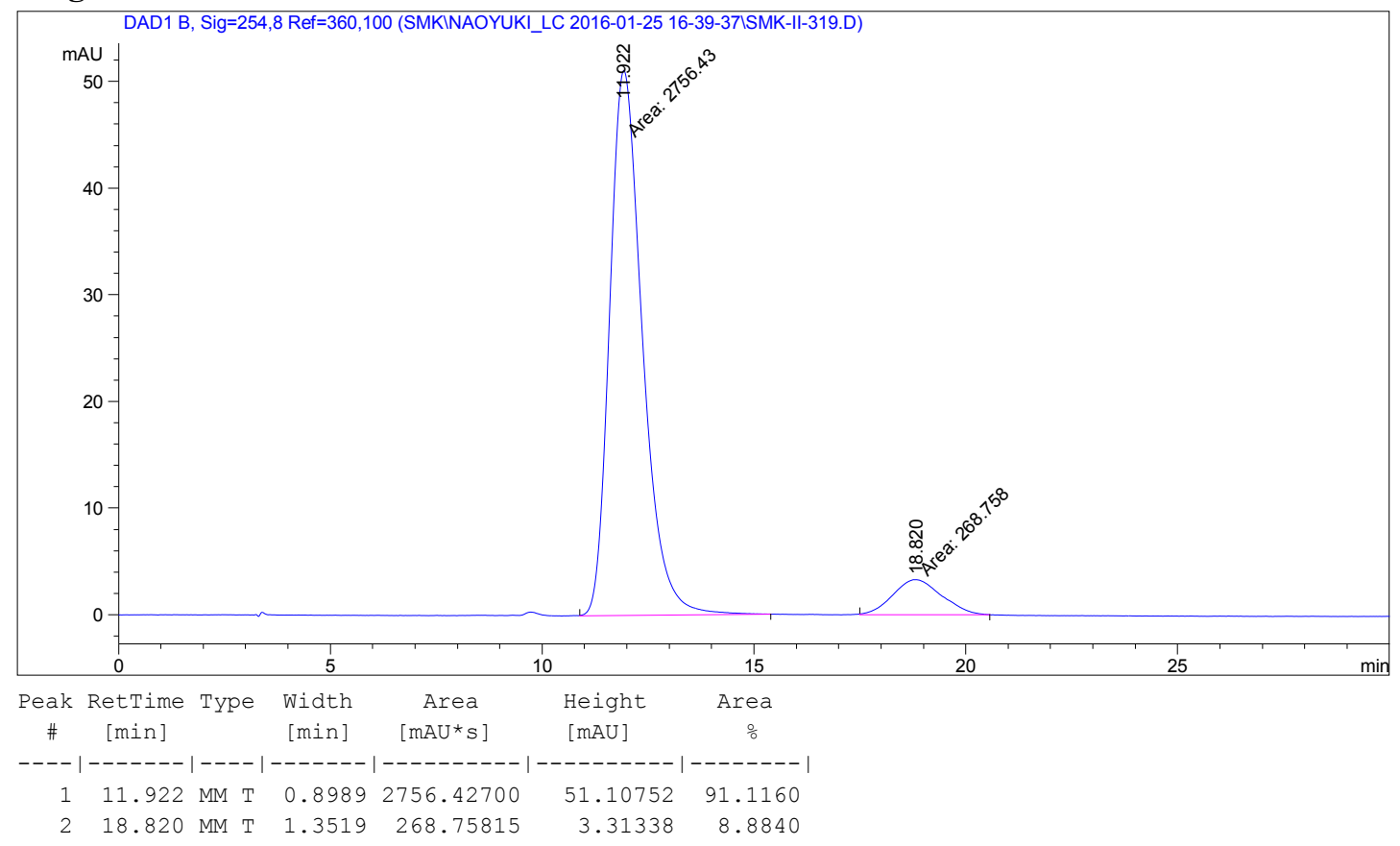




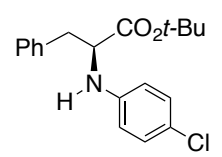

$8 \mathrm{~h}$

HPLC analysis (OJ-H, 5\% IPA-hexanes, $0.8 \mathrm{~mL} / \mathrm{min}, 254 \mathrm{~nm})$ indicated $91 \%$ ee: tR (minor) $=$ 15.7 min, $\mathrm{tR}$ (major) $=19.8$ min.

\section{DL-8h}

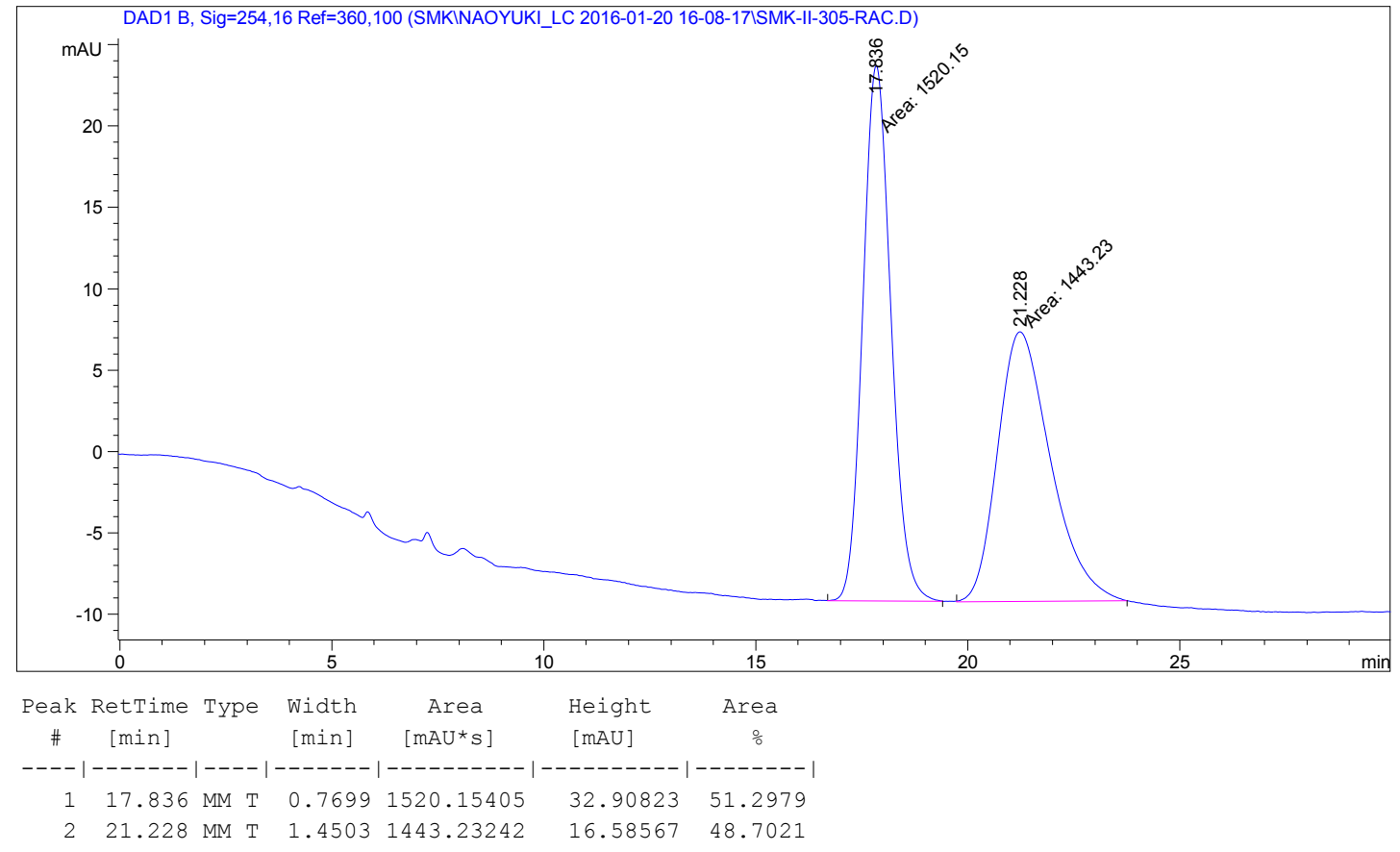

L-8h: $91 \%$ ee

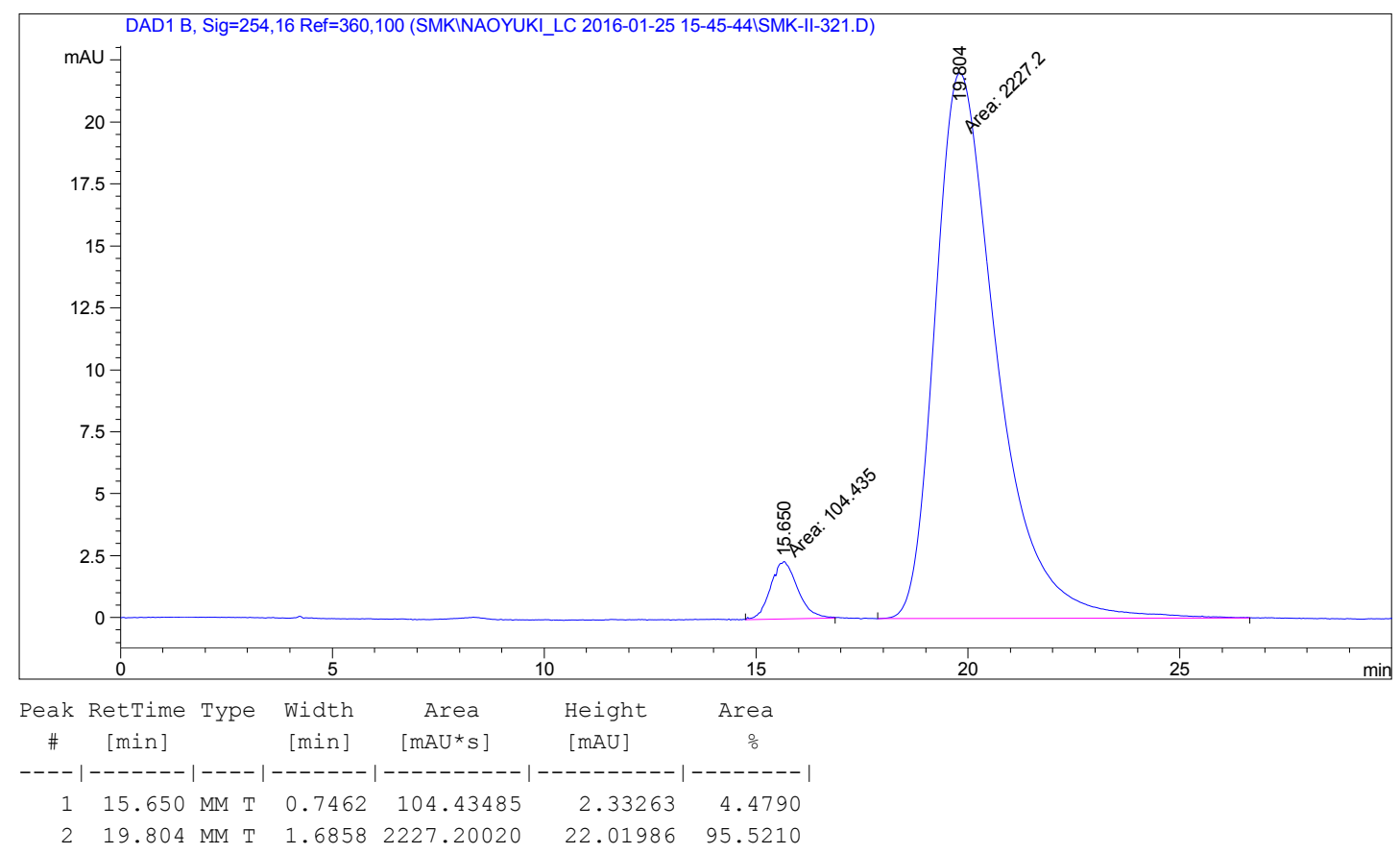




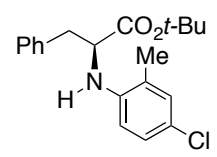

$8 \mathbf{i}$

HPLC analysis (OJ-H, 2\% IPA-hexanes, $0.8 \mathrm{~mL} / \mathrm{min}, 254 \mathrm{~nm})$ indicated $88 \%$ ee: tR (minor) $=$ $11.9 \mathrm{~min}, \mathrm{tR}$ (major) $=18.0 \mathrm{~min}$.

\section{DL-8i}

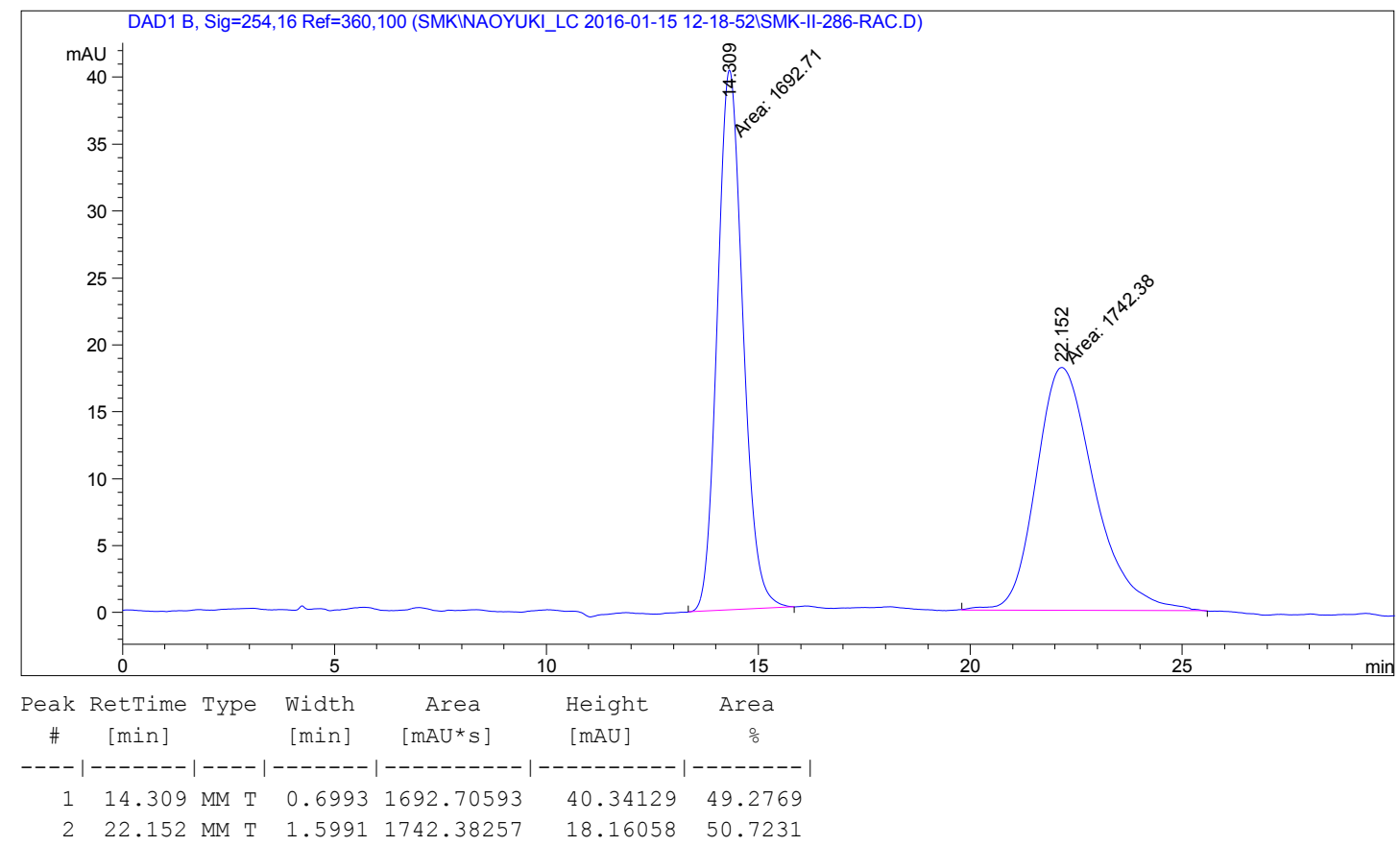

L-8i: $88 \%$

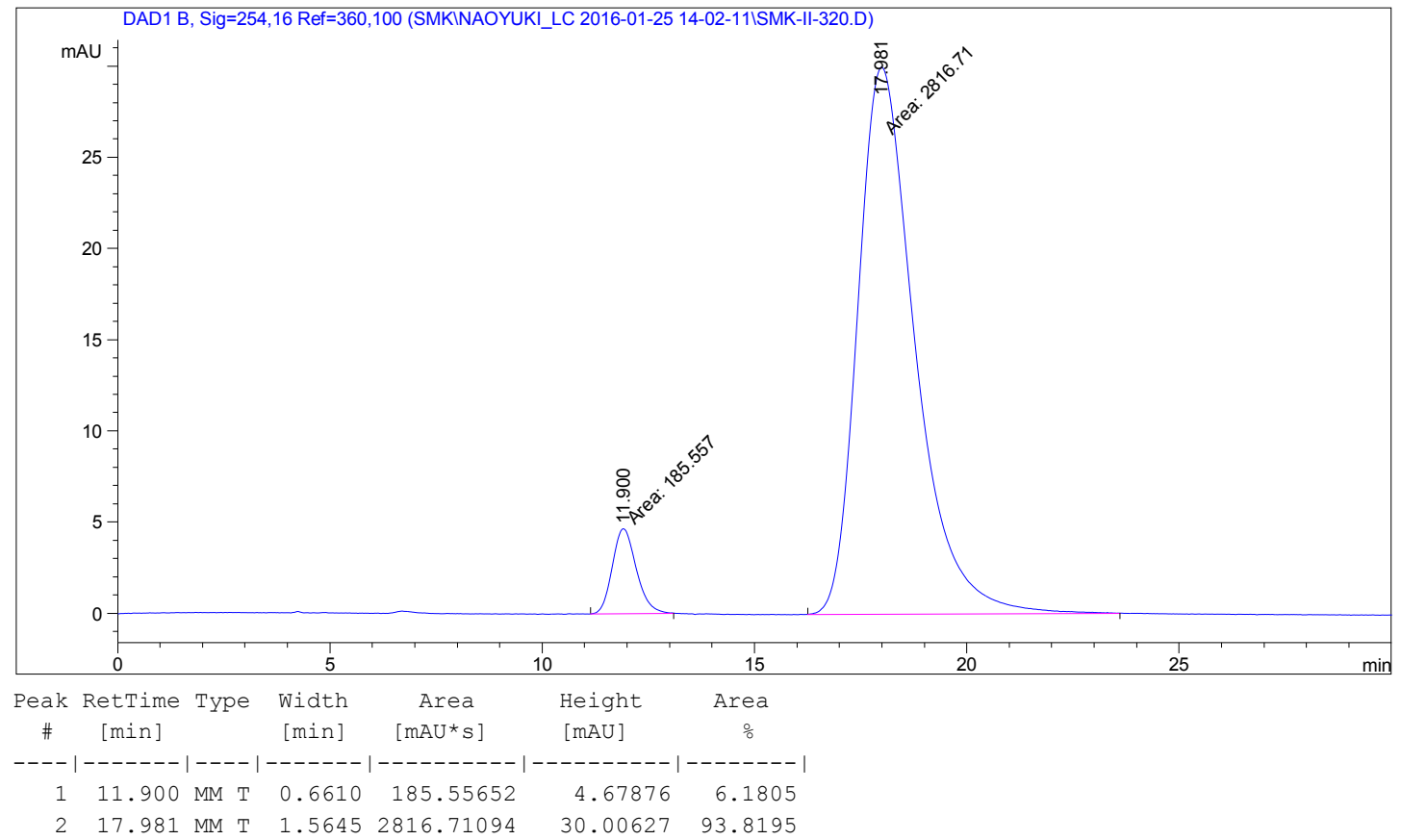




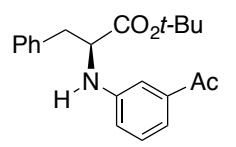

8j

HPLC analysis (AD-H, 10\% IPA-hexanes, $1.0 \mathrm{~mL} / \mathrm{min}, 254 \mathrm{~nm}$ ) indicated $85 \%$ ee: $\mathrm{tR}$ (minor) = $13.9 \mathrm{~min}, \mathrm{tR}$ (major) $=20.2 \mathrm{~min}$.

\section{DL-8j}

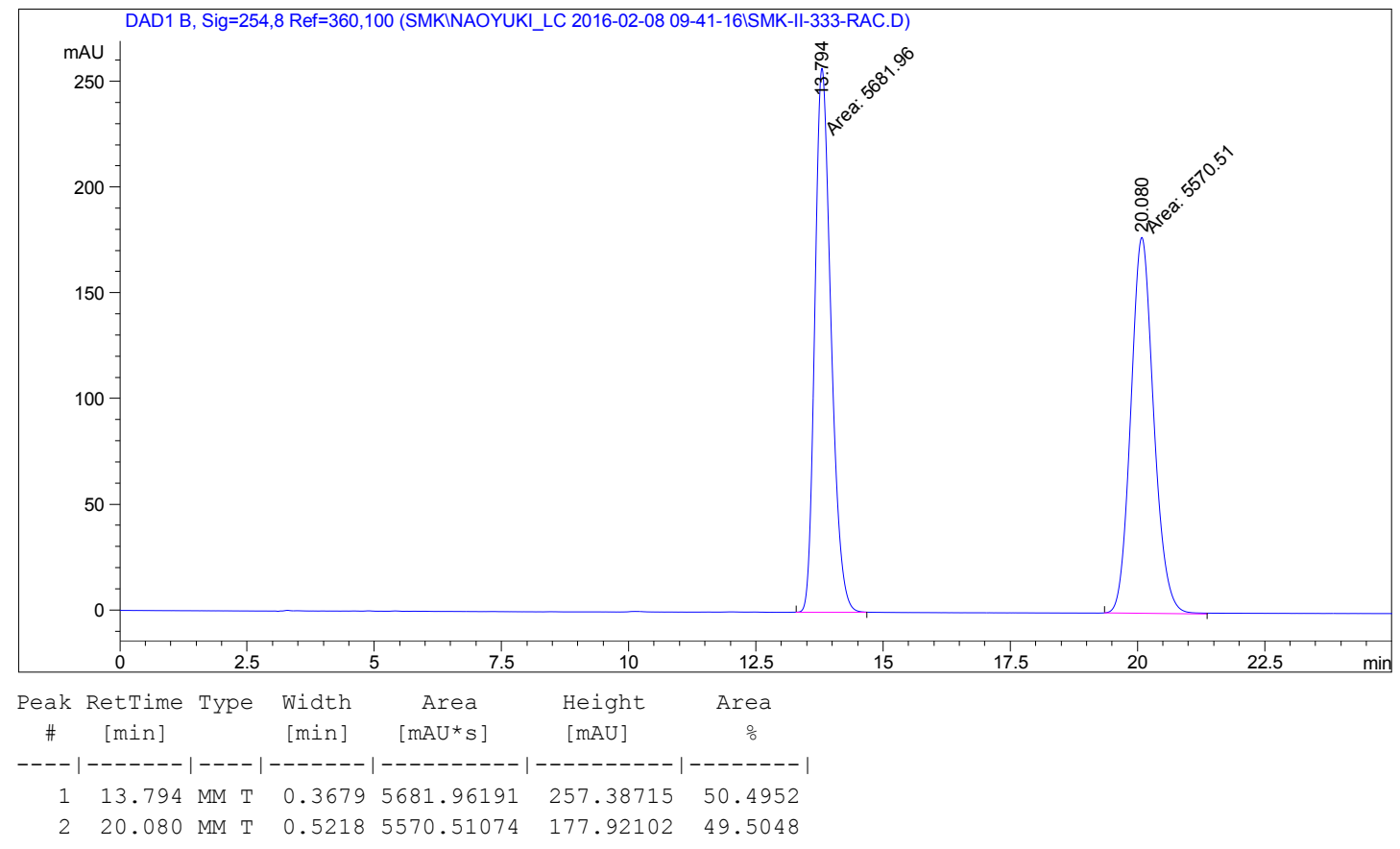

L-8j: 85\% ee

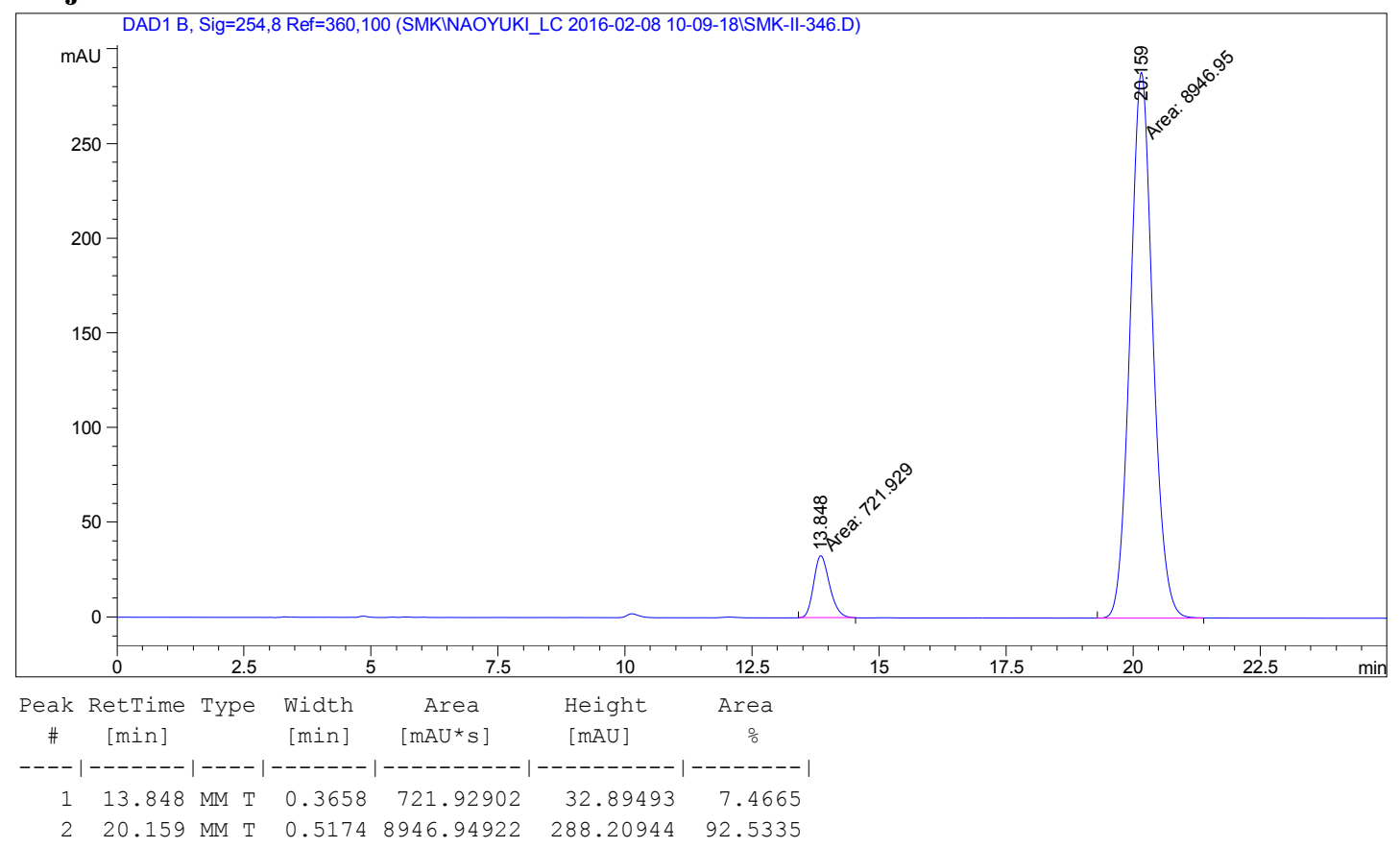




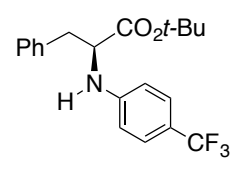

$8 k$

HPLC analysis (OJ-H, 2\% IPA-hexanes, $0.8 \mathrm{~mL} / \mathrm{min}, 254 \mathrm{~nm}$ ) indicated 55\% ee: tR (minor) = $18.4 \mathrm{~min}, \mathrm{tR}$ (major) $=24.4 \mathrm{~min}$.

\section{DL-8k}

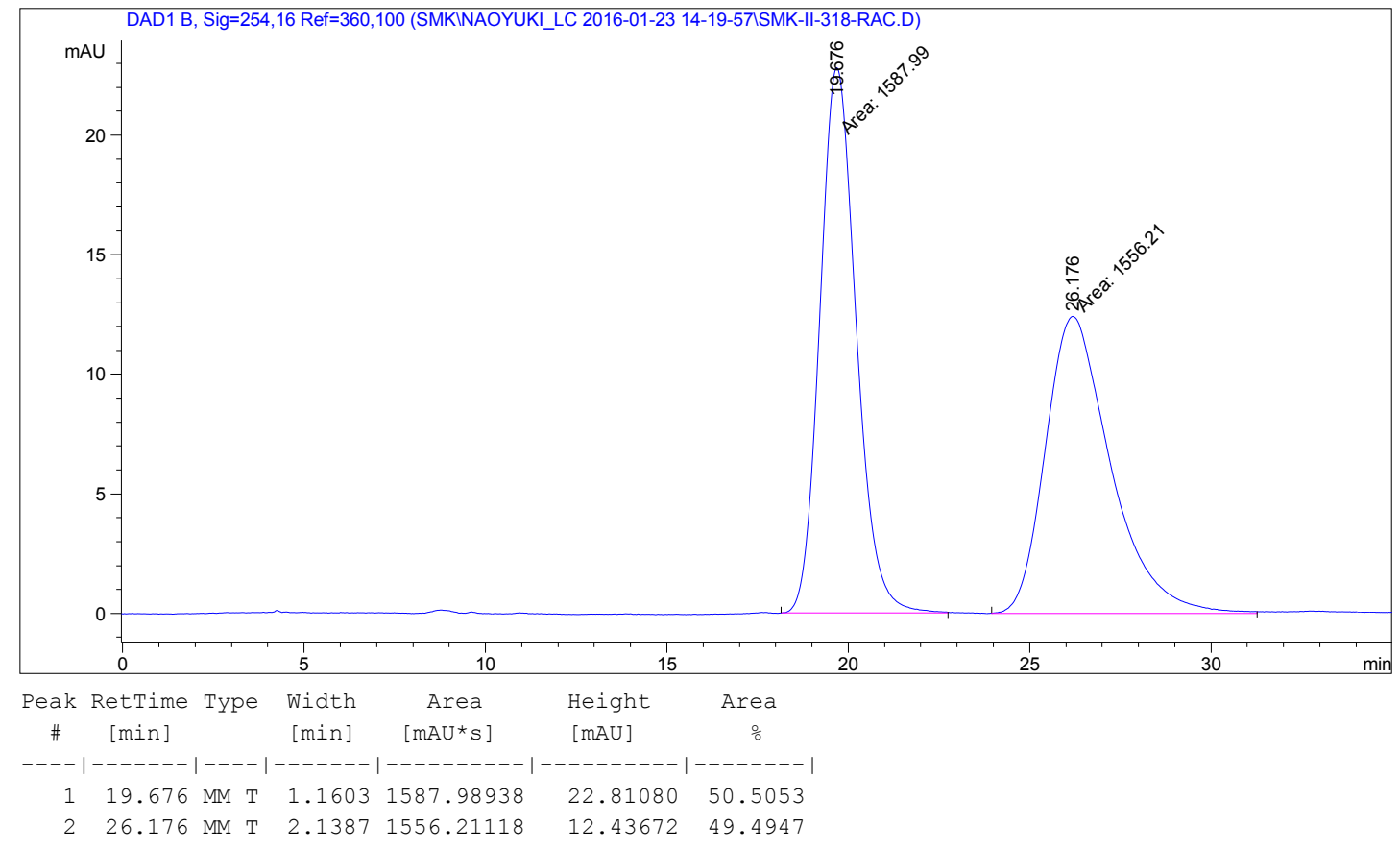

L-8k: $55 \%$ ee

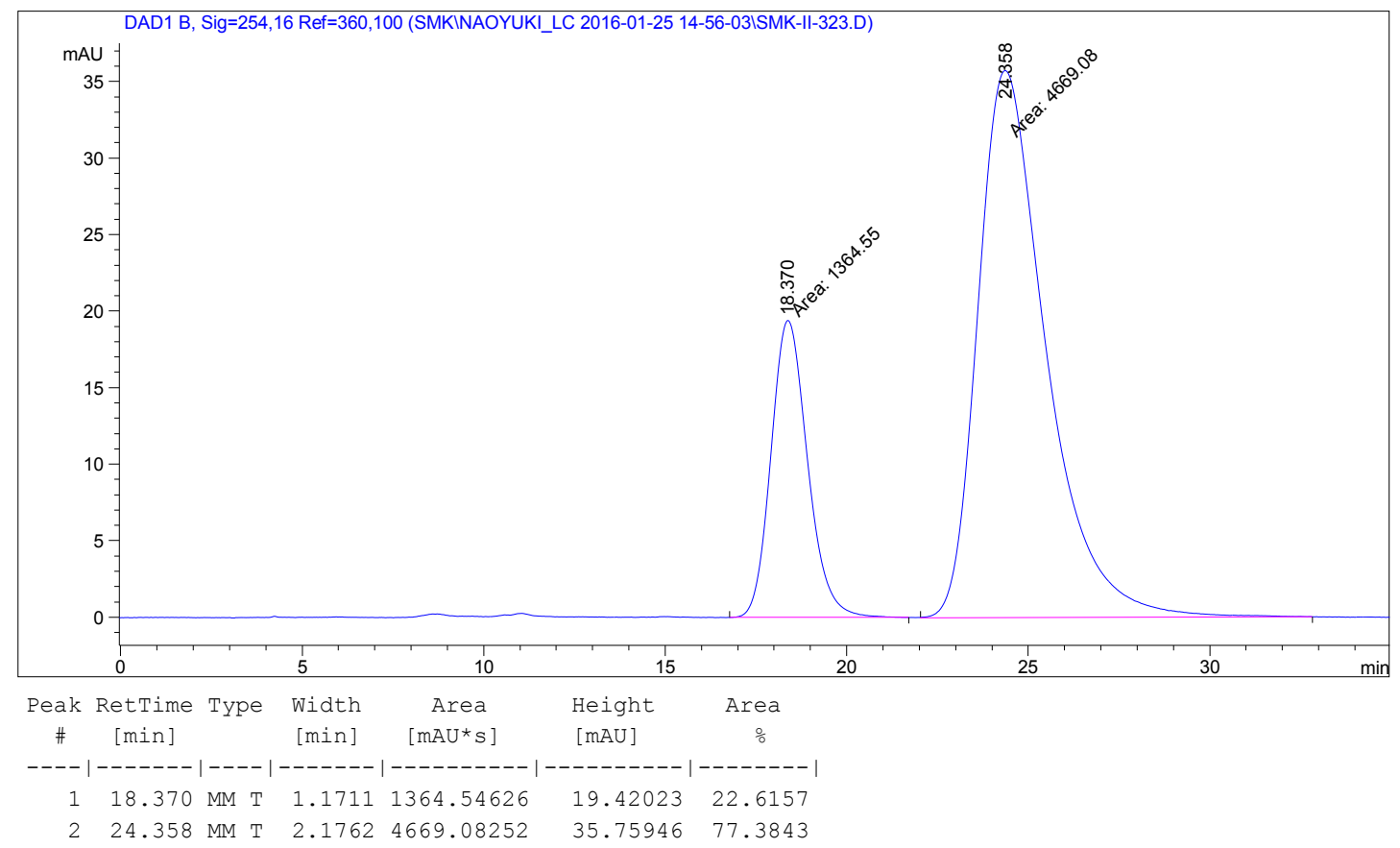




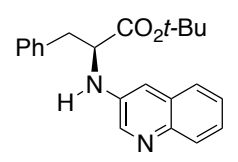

81

HPLC analysis (AD-H, 10\% IPA-hexanes, $1.0 \mathrm{~mL} / \mathrm{min}, 254 \mathrm{~nm}$ ) indicated $97 \%$ ee: $\mathrm{tR}$ (minor) = $12.3 \mathrm{~min}$, tR (major) $=25.7 \mathrm{~min}$.

\section{DL-81}

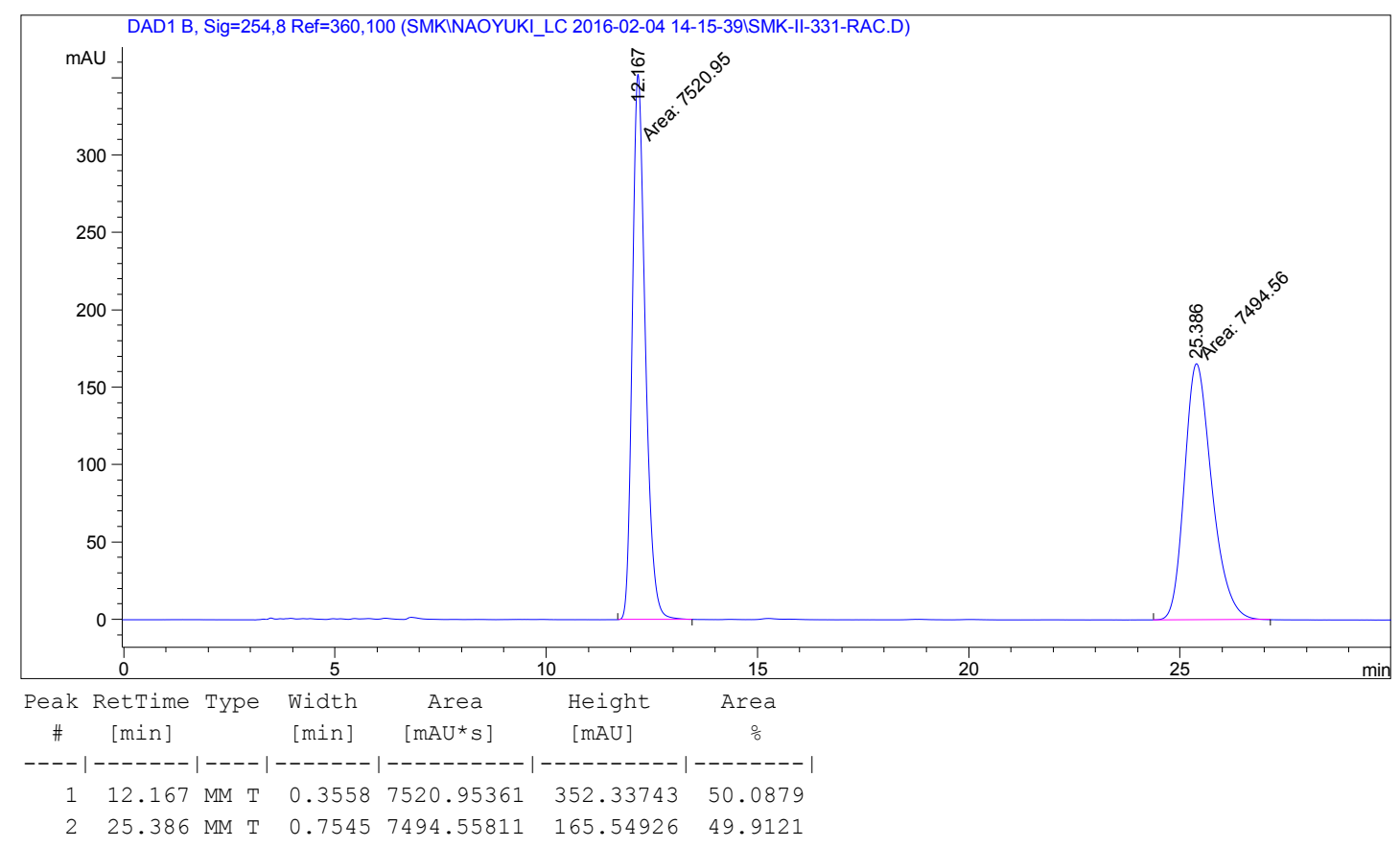

L-81: $97 \%$ ee

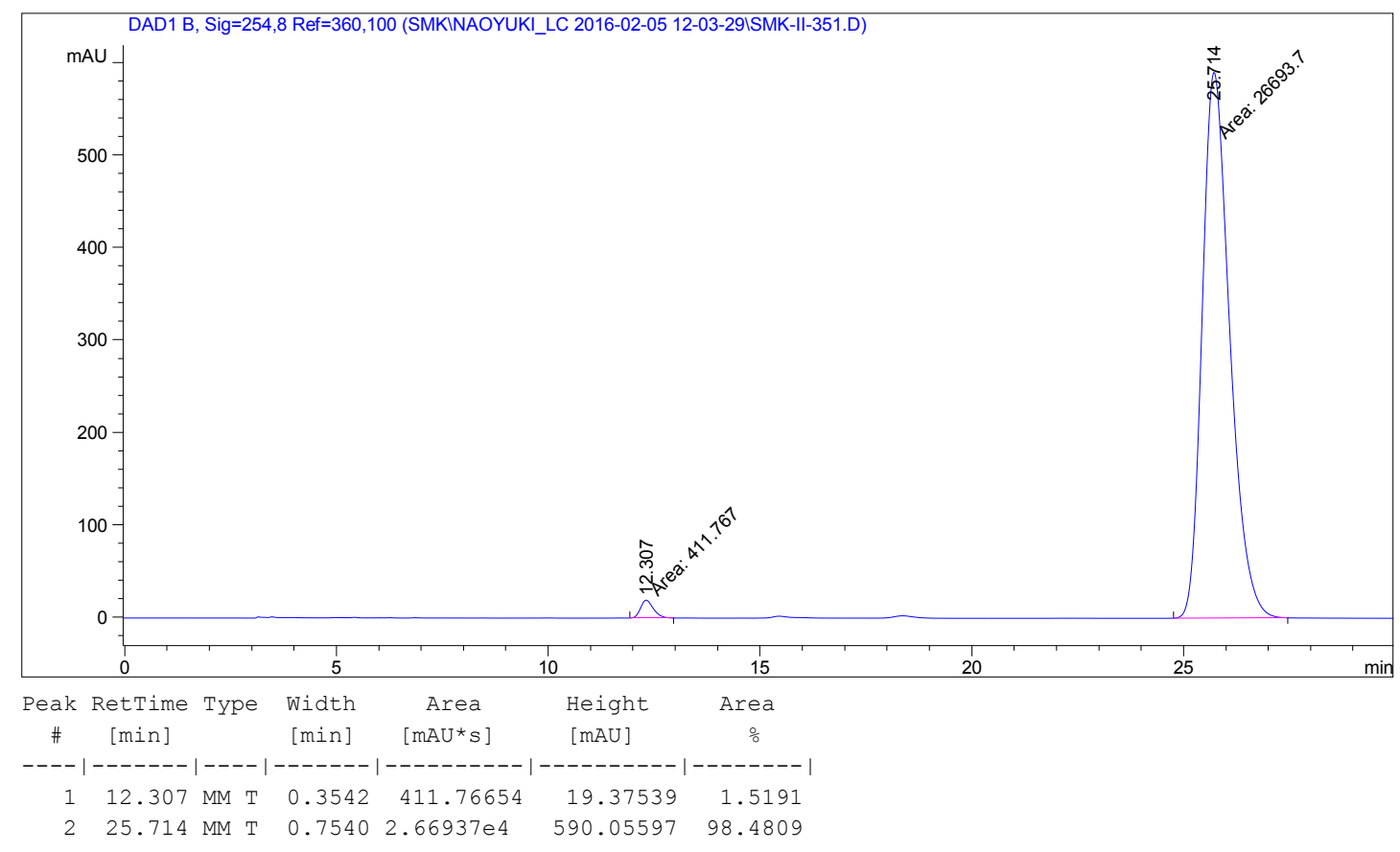


Table S1. Summary of initial $N$-arylation experiments.

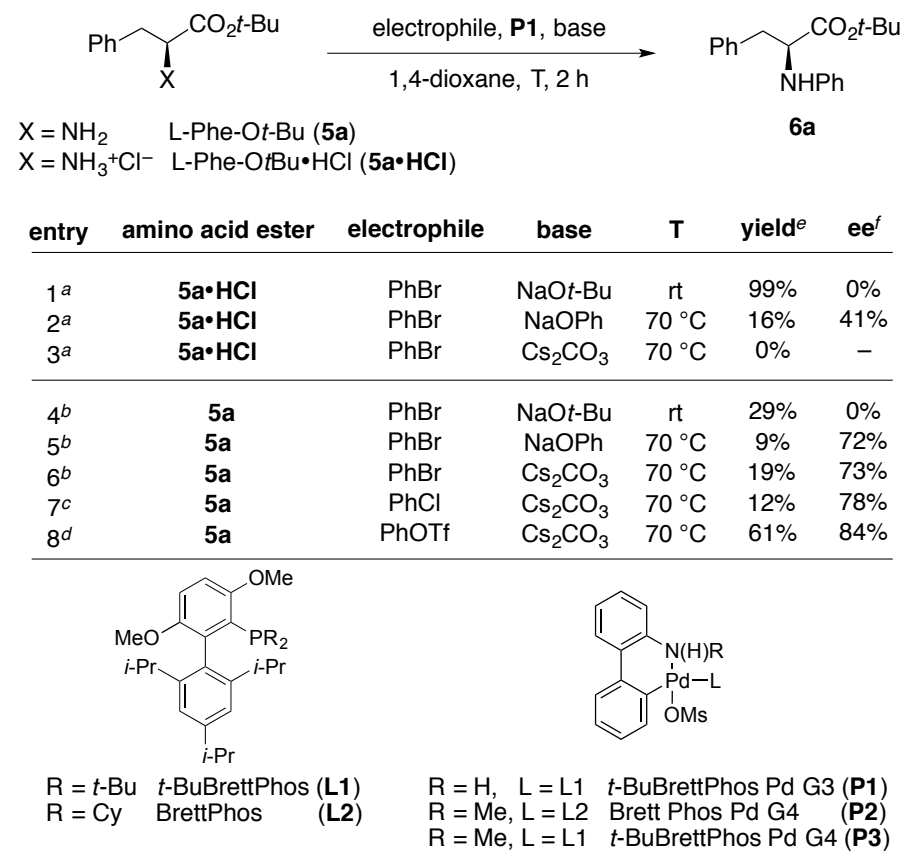

${ }^{a}$ Reaction Conditions: L-Phe-Ot-Bu・HCl $(\mathbf{5 a} \bullet \mathbf{H C l}, 1.2$ equiv), base (2.4 equiv), bromobenzene (1 equiv), P1 (1 mol\%). ${ }^{b}$ Reaction Conditions: L-Phe-Ot-Bu (5a, 1.2 equiv), base (1.2 equiv), bromobenzene (1 equiv), P1 (1 mol\%). ${ }^{c}$ Reaction Conditions: L-Phe-Ot-Bu (5a, 1.2 equiv), base (1.2 equiv), chlorobenzene (1 equiv), P1 (1 mol\%). ${ }^{d}$ Reaction Conditions: L-Phe-Ot-Bu (5a, 1.2 equiv), base (1.2 equiv), phenyl trifluoromethanesulfonate (1 equiv), P1 (1 mol\%). ${ }^{e}$ Isolated yields. ${ }^{f}$ Enantiomeric excess (ee) was determined by HPLC analysis using chiral stationary phases.<smiles>CC(C)(C)OC(=O)C(Cc1ccccc1)Nc1ccccc1</smiles>

6a

HPLC analysis conditions: OJ-H, 2\% IPA-hexanes, $0.8 \mathrm{~mL} / \mathrm{min}, 254 \mathrm{~nm}$

L-6a entry 1: 0\% ee

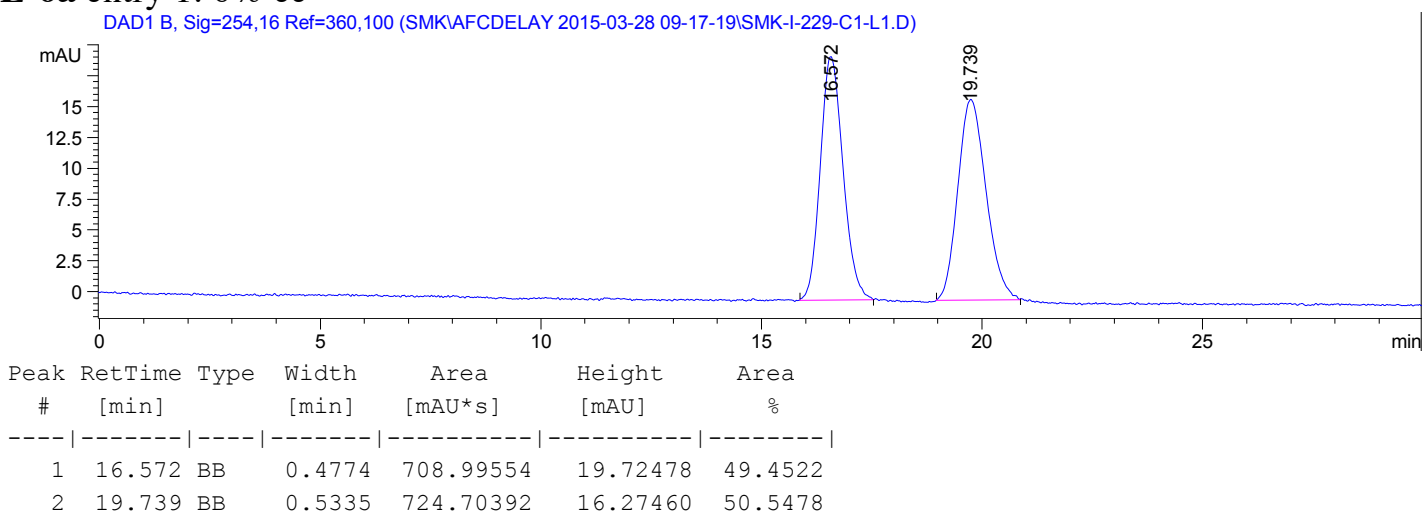


L-6a entry 2: 41\% ee

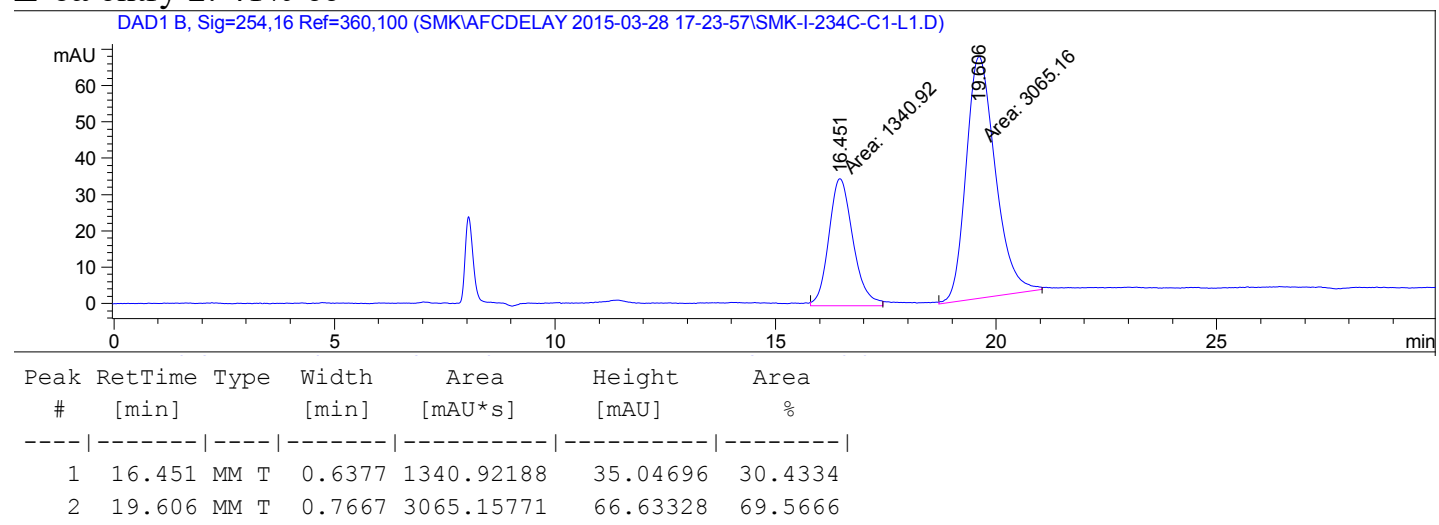

L-6a entry 4: 0\% ee

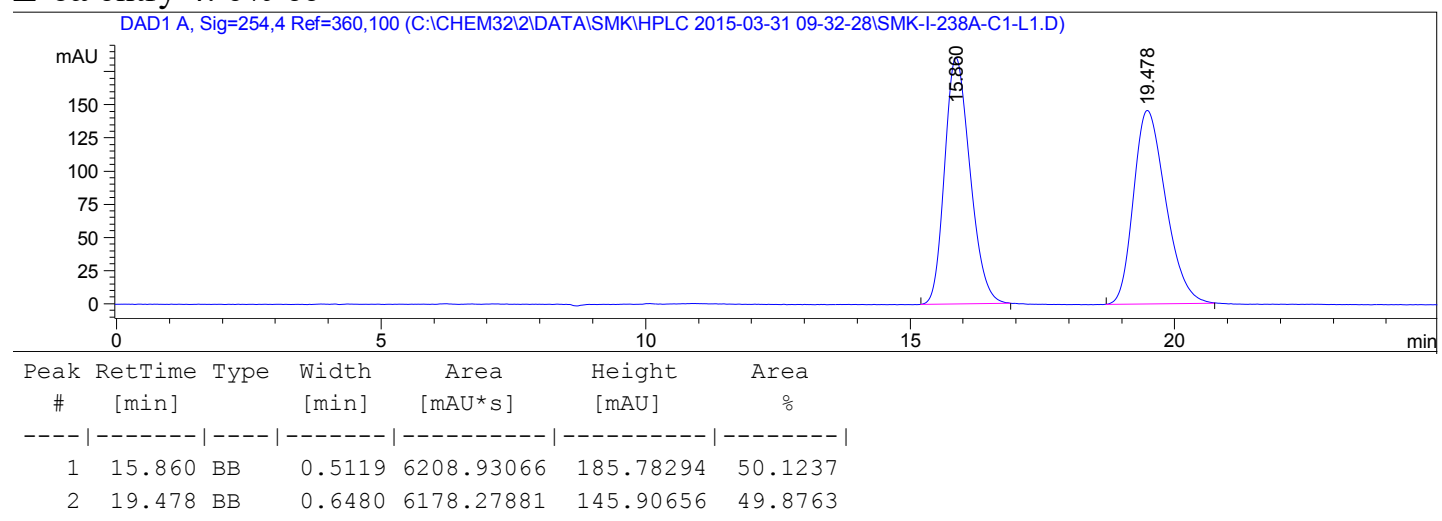

L-6a entry 5: 72\% ee

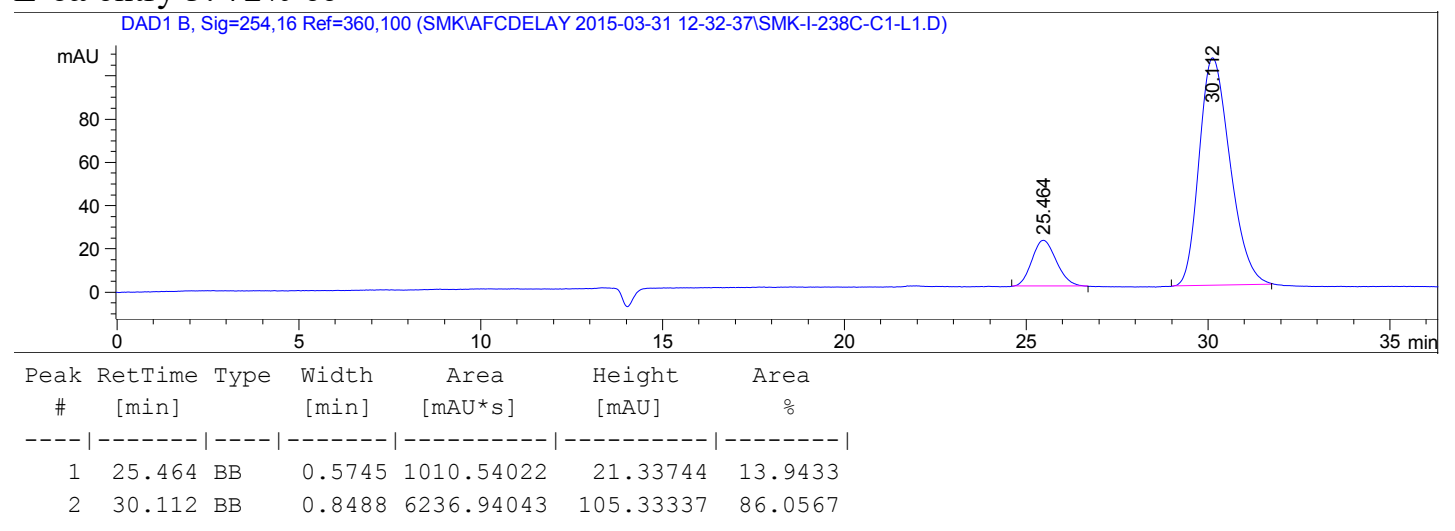


L-6a entry 6: 73\% ee

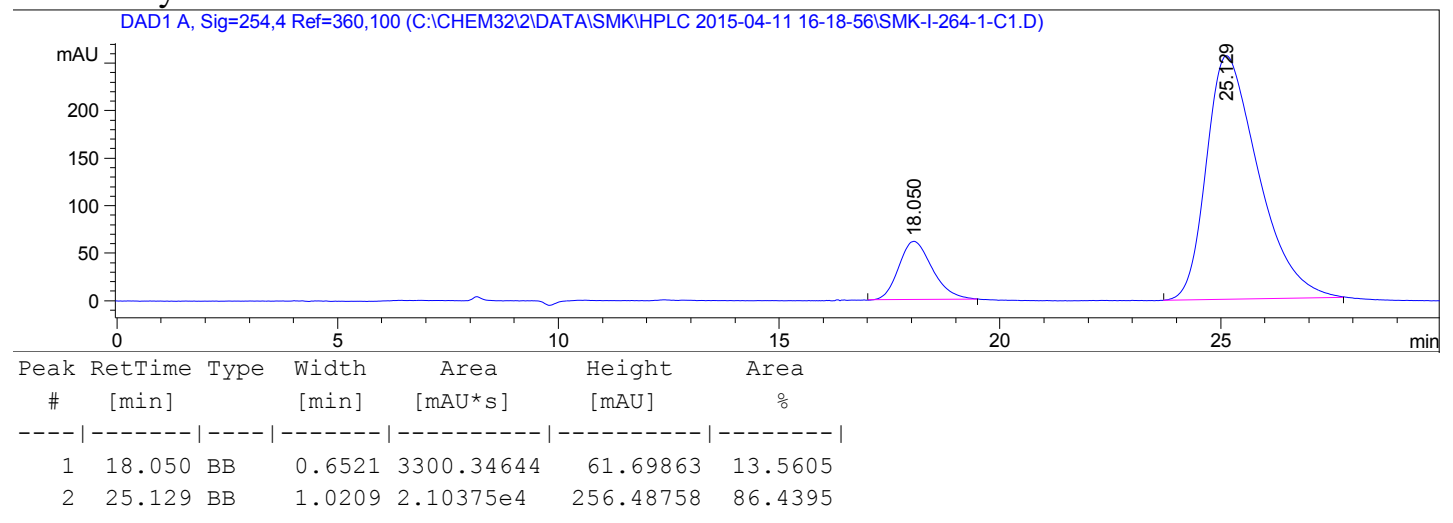

L-6a entry 7: 78\% ee

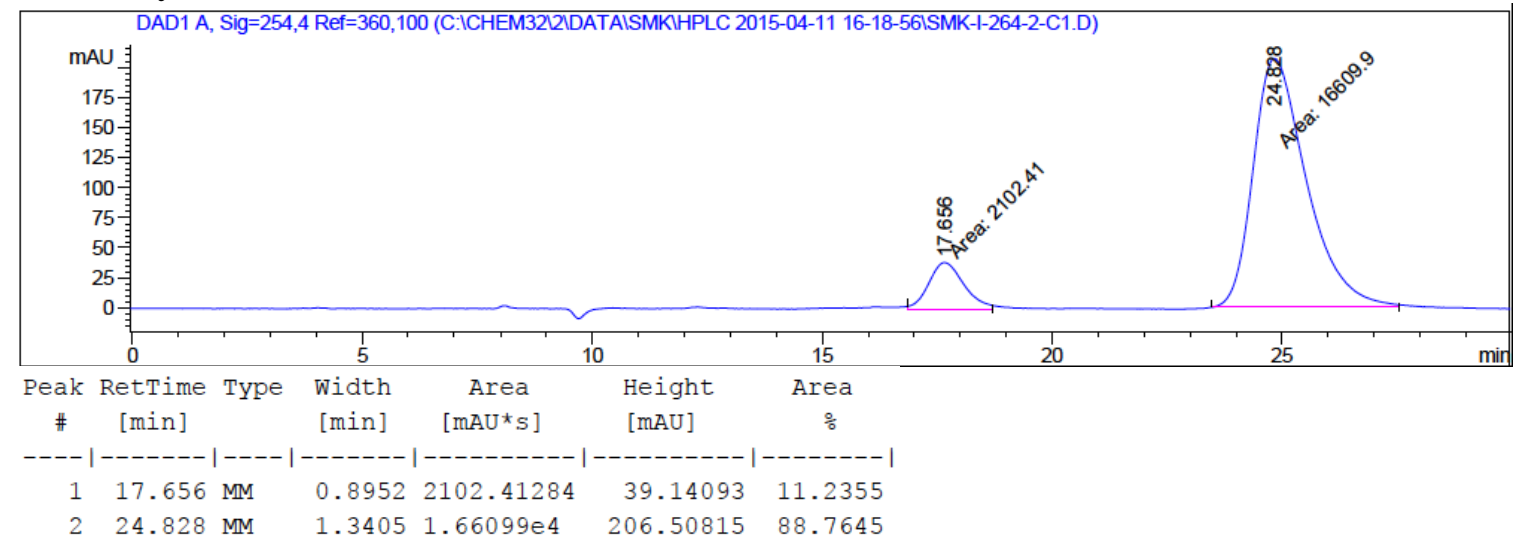

L-6a entry 8: 84\% ee

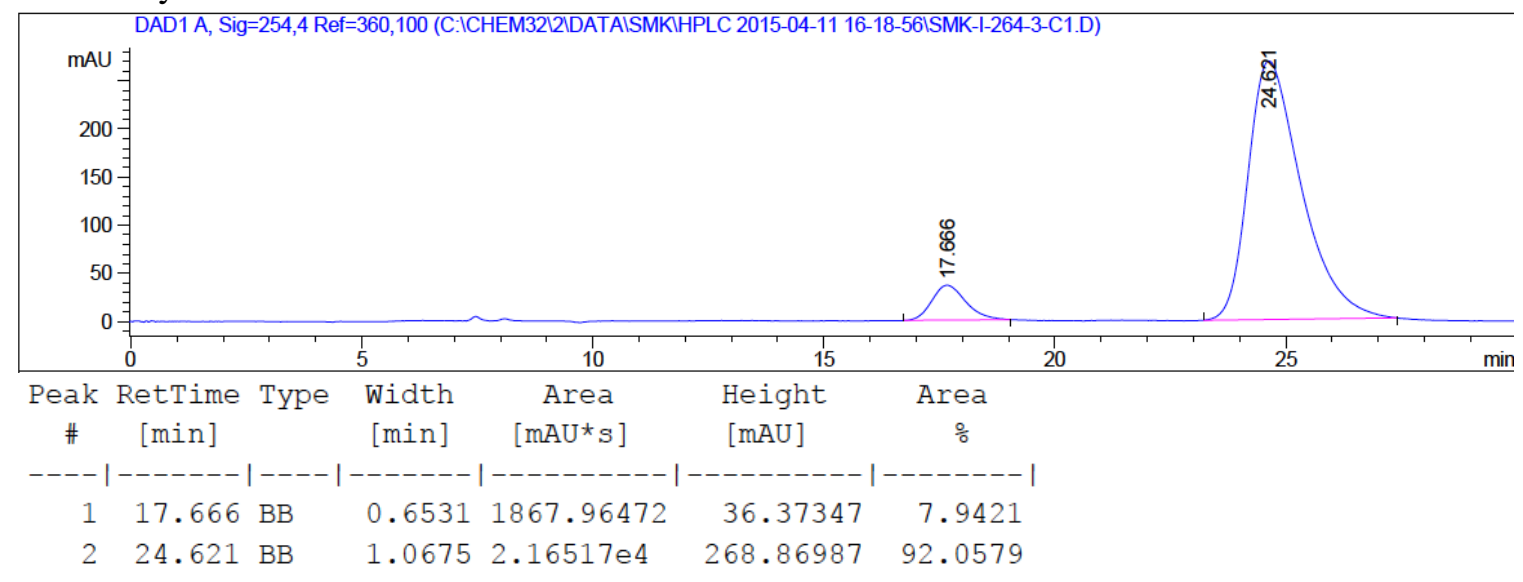




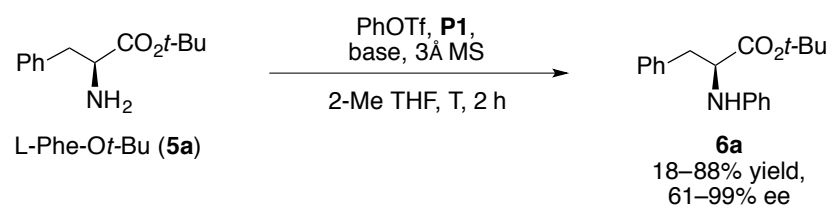

Table S3. Subset of reactions from subsequent DOE analysis.

\begin{tabular}{ccccccccc}
\hline & & \multicolumn{8}{c}{ 2 mol\% precatalyst } & $\mathbf{5}$ mol\% precatalyst \\
\hline & & base & $\begin{array}{c}\text { equiv base } \\
\text { (to 5a) }\end{array}$ & $\mathbf{3} \AA \mathbf{~ M S}$ & yield & ee & yield & ee \\
\hline 1 & $50{ }^{\circ} \mathrm{C}$ & $\mathrm{Cs}_{2} \mathrm{CO}_{3}$ & 1 & $0 \mathrm{mg}$ & $64 \%$ & $91 \%$ & $89 \%$ & $94 \%$ \\
2 & $50{ }^{\circ} \mathrm{C}$ & $\mathrm{Cs}_{2} \mathrm{CO}_{3}$ & 1 & $50 \mathrm{mg}$ & $51 \%$ & $95 \%$ & $80 \%$ & $92 \%$ \\
3 & $50{ }^{\circ} \mathrm{C}$ & $\mathrm{Cs}_{2} \mathrm{CO}_{3}$ & 3 & $0 \mathrm{mg}$ & $69 \%$ & $89 \%$ & $93 \%$ & $91 \%$ \\
4 & $50{ }^{\circ} \mathrm{C}$ & $\mathrm{Cs}_{2} \mathrm{CO}_{3}$ & 3 & $50 \mathrm{mg}$ & $69 \%$ & $92 \%$ & $95 \%$ & $94 \%$ \\
\hline
\end{tabular}

Yellow indicates optimized reaction conditions.<smiles>CC(C)(C)OC(=O)C(Cc1ccccc1)Nc1ccccc1</smiles>

6a

HPLC analysis conditions: OJ-H, 2\% IPA-hexanes, $0.8 \mathrm{~mL} / \mathrm{min}, 254 \mathrm{~nm}$

\section{DL-6a}

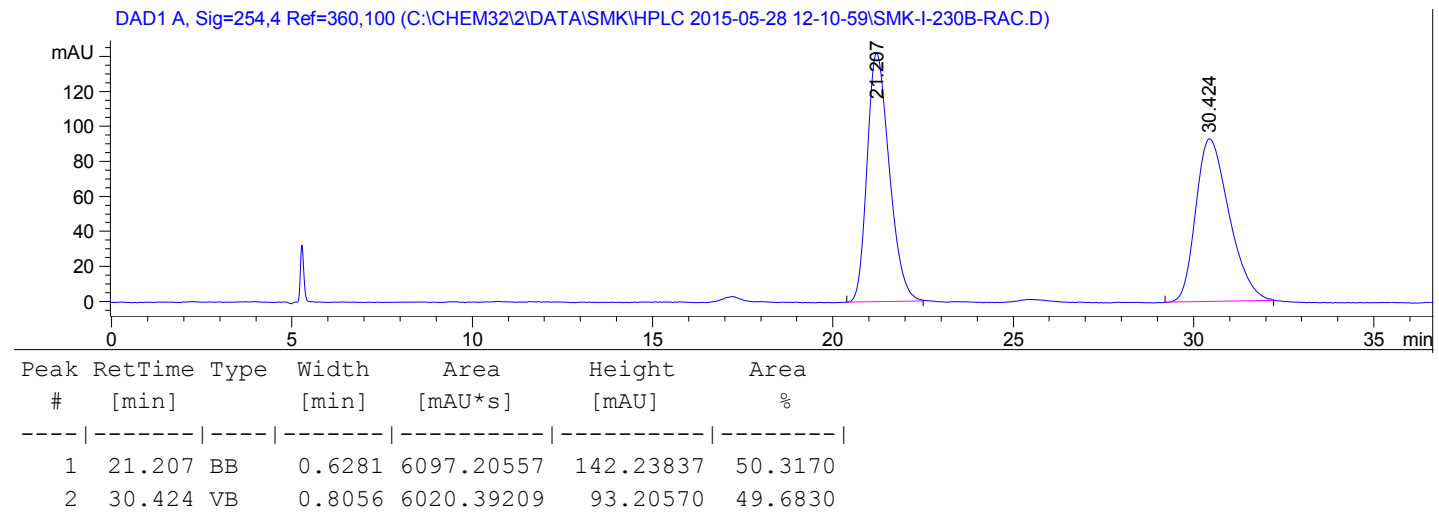

L-6a entry 1, 2 mol\% precatalyst: $91 \%$ ee

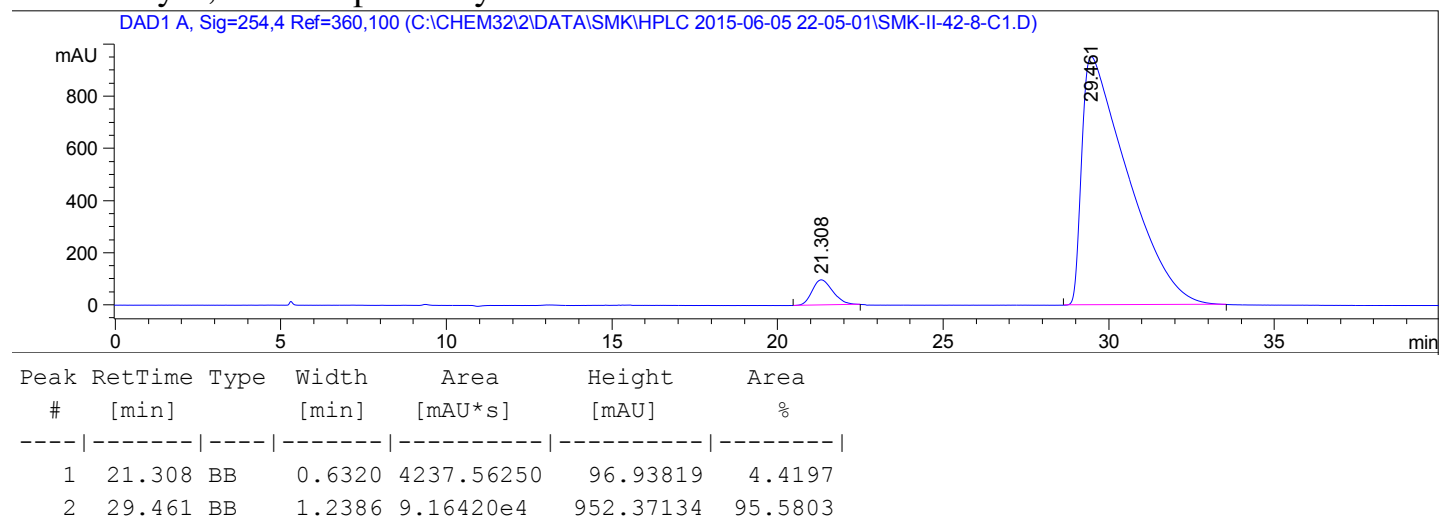


L-6a entry 2, 2 mol\% precatalyst: $95 \%$ ee

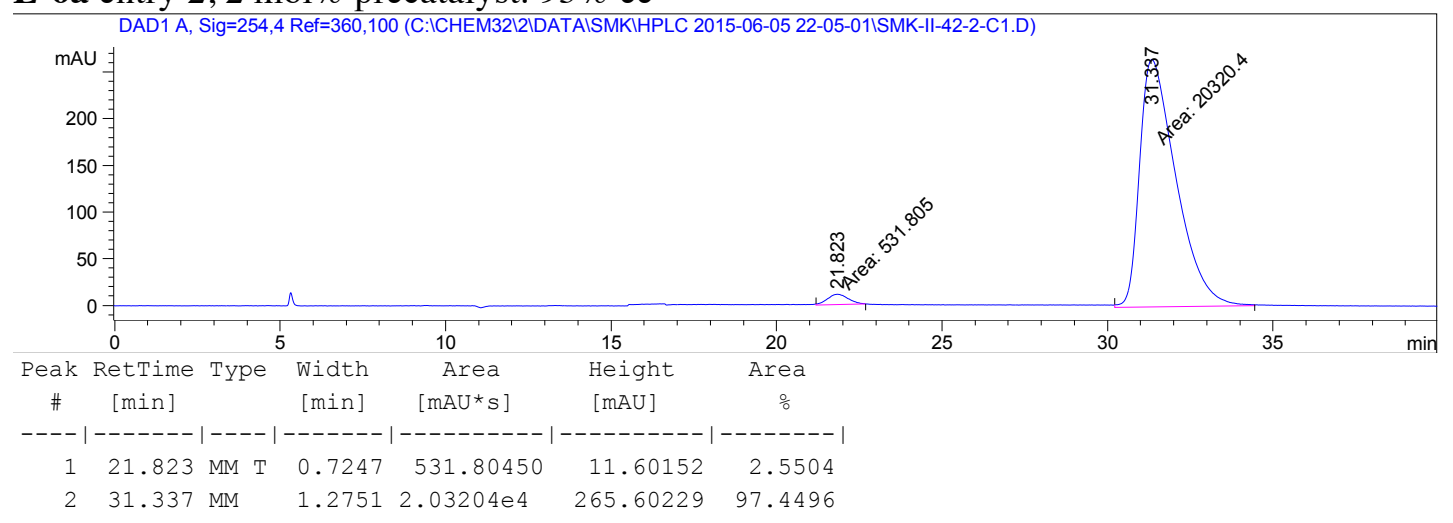

L-6a entry 3, 2 mol\% precatalyst: $89 \%$ ee

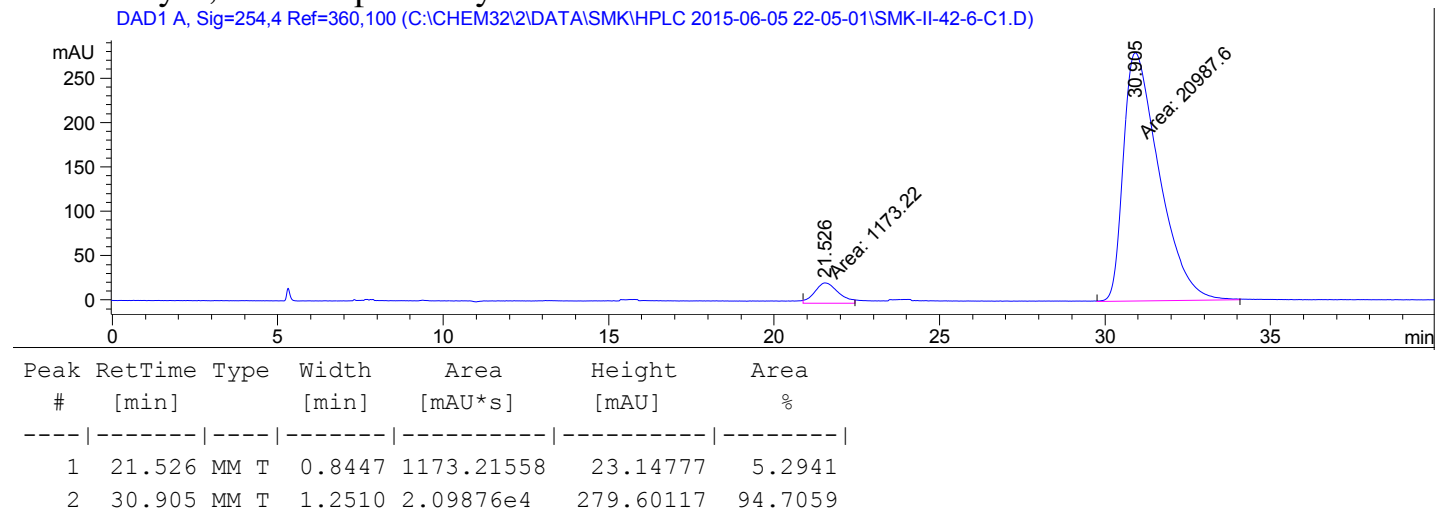

L-6a entry 4, 2 mol\% precatalyst: $92 \%$ ee

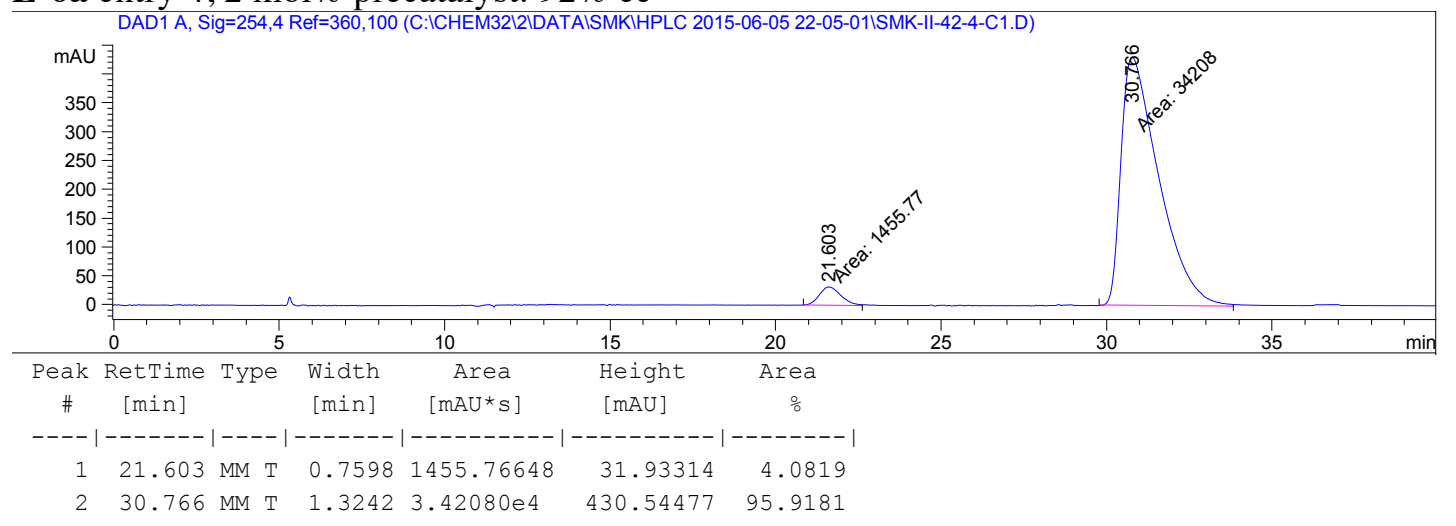


$\mathrm{Ph} \overbrace{\mathrm{NHPh}}^{\mathrm{CO}_{2} t-\mathrm{Bu}}$

6a

HPLC analysis conditions: OJ-H, 2\% IPA-hexanes, $0.8 \mathrm{~mL} / \mathrm{min}, 254 \mathrm{~nm}$

\section{DL-6a}

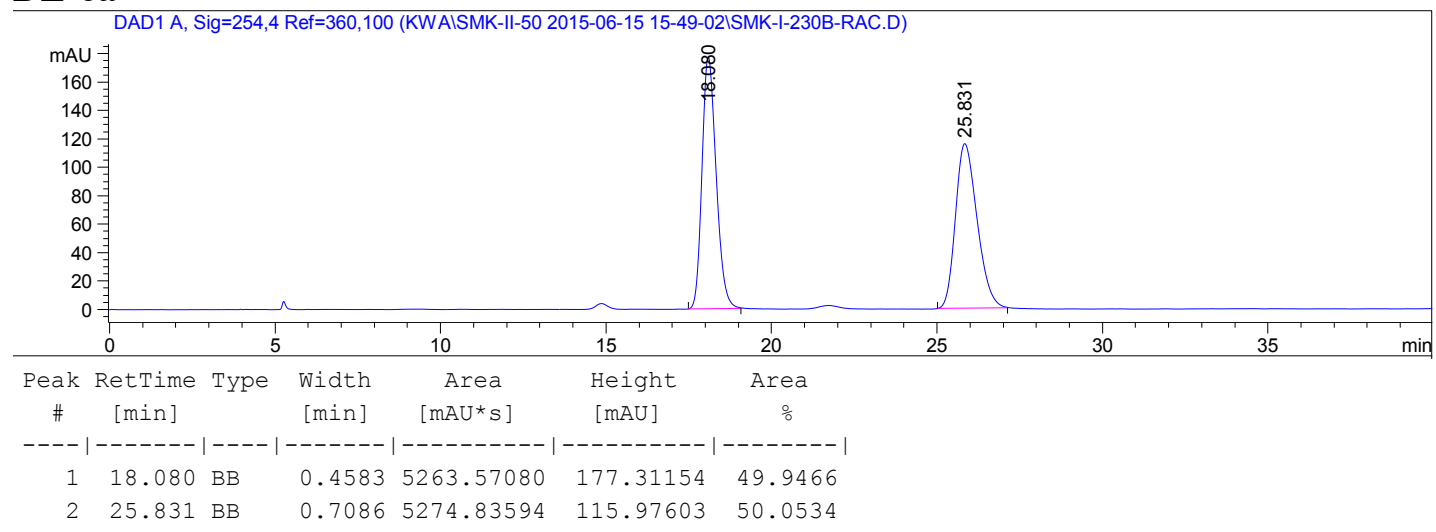

L-6a entry 1, 5 mol\% precatalyst: $94 \%$ ee

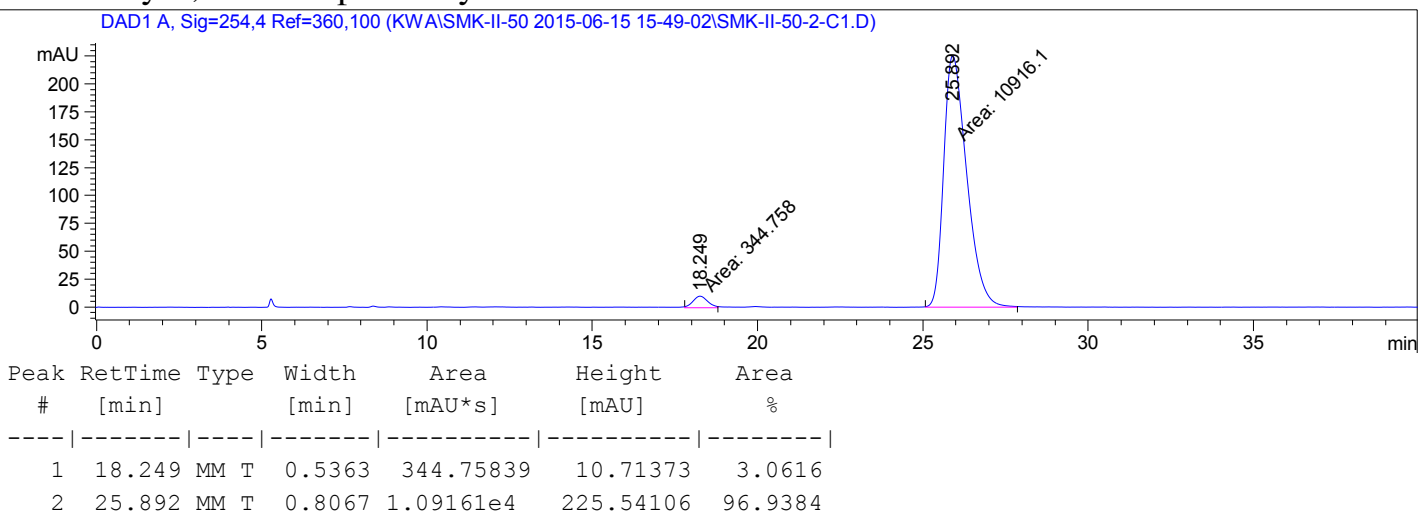

L-6a entry 2, 5 mol\% precatalyst: $92 \%$ ee

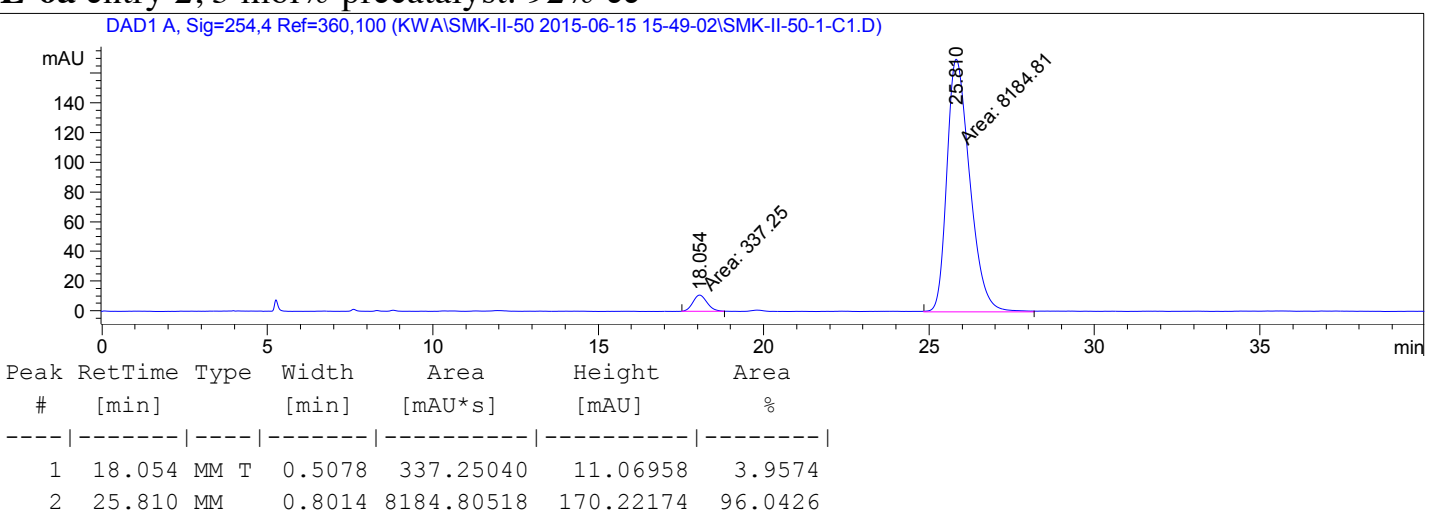


L-6a entry 3, 5 mol\% precatalyst: $91 \%$ ee

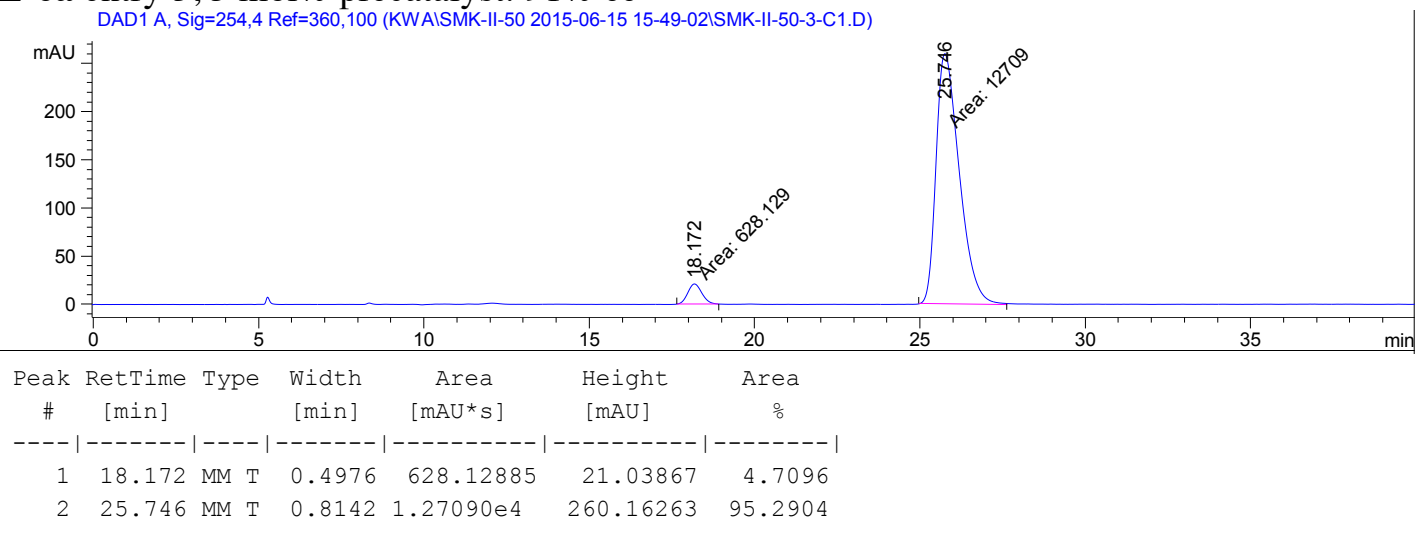

L-6a entry 4, 5 mol\% precatalyst: $94 \%$ ee

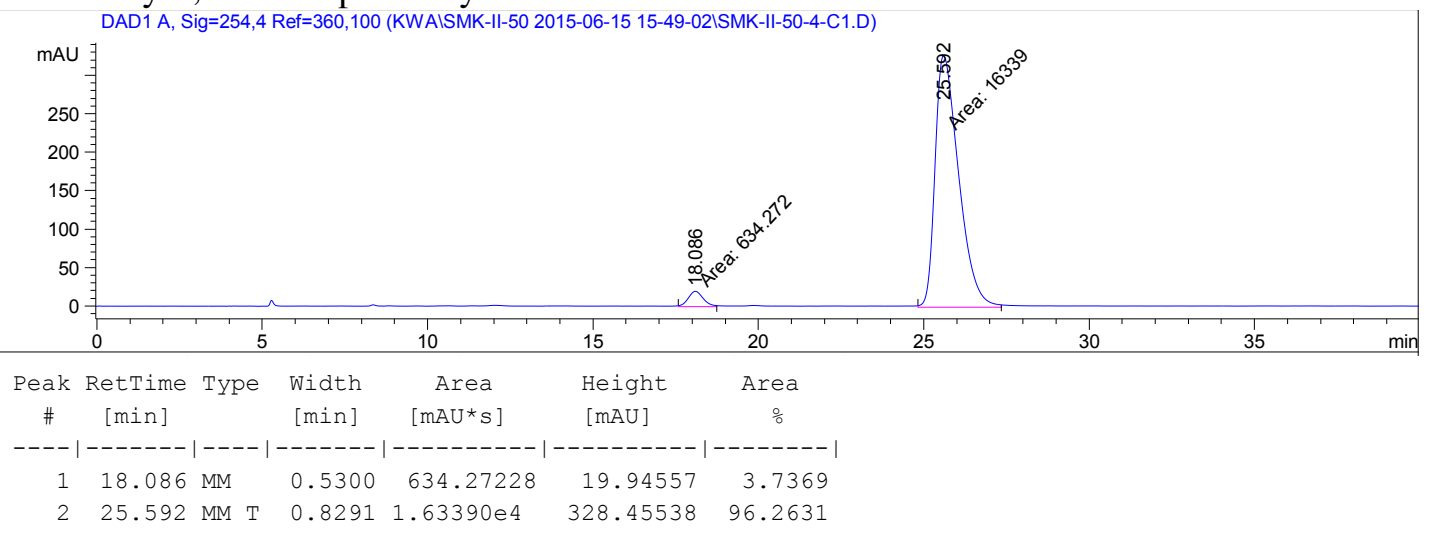


A.

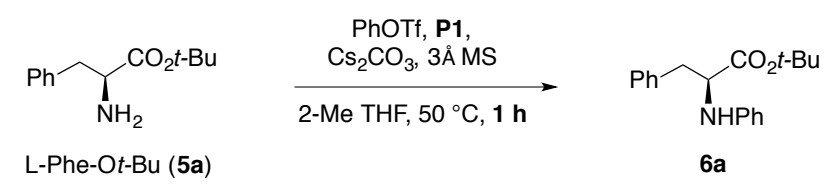

\begin{tabular}{ccc} 
& ee before reaction & ee after reaction \\
& \\
\hline $\mathbf{5 a}$ & $99 \%$ & $81 \%$ \\
$\mathbf{6 a}$ & - & $97 \%$ \\
\hline${ }^{a}$ Enantiomeric excess (ee) was determined directly from the crude reaction mixture by \\
HPLC analysis using chiral stationary phases.
\end{tabular}

Scheme 3. A. Experiment determining the enantiomeric excess before and after the reaction.

L-Phe-Ot-Bu (5a)

HPLC analysis conditions: AD-H, 1\% IPA-hexanes, $0.8 \mathrm{~mL} / \mathrm{min}, 210 \mathrm{~nm}$

\section{DL-Phe-O $t$-Bu (5a)}

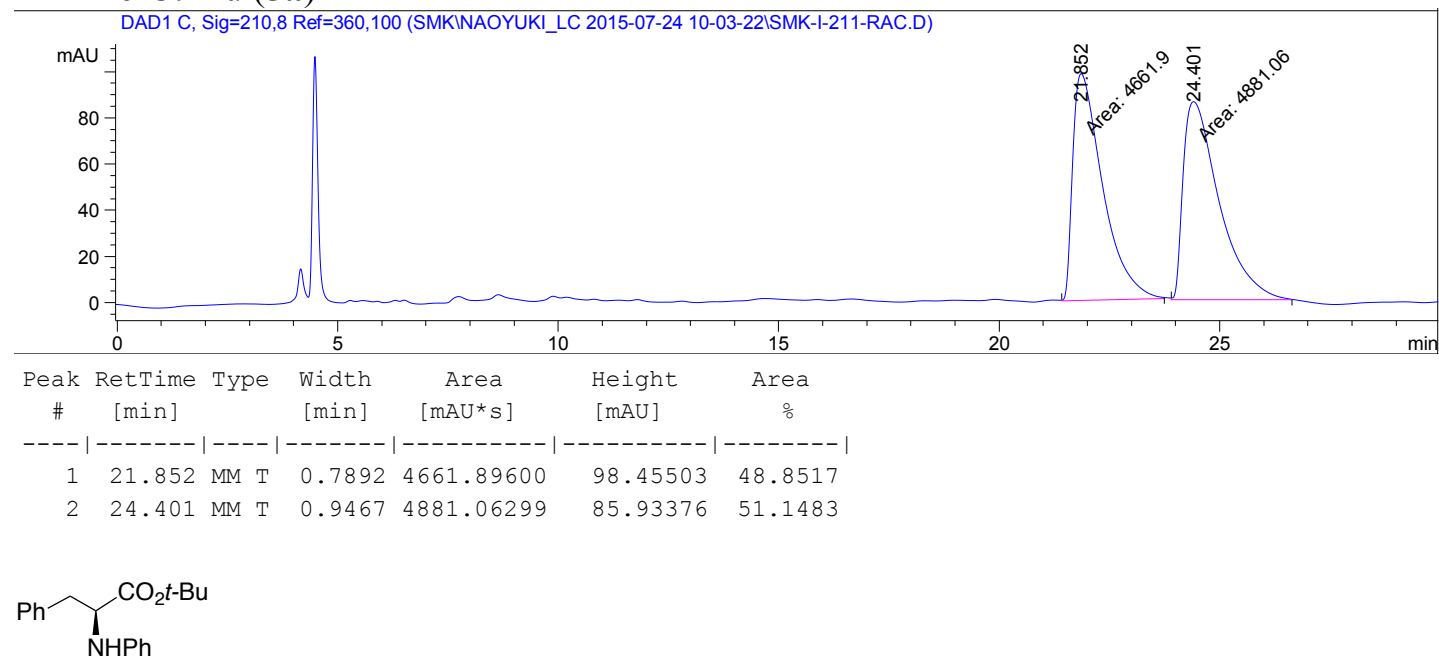

$6 a$

HPLC analysis conditions: AD-H, 1\% IPA-hexanes, $0.8 \mathrm{~mL} / \mathrm{min}, 254 \mathrm{~nm}$

\section{DL-6a}

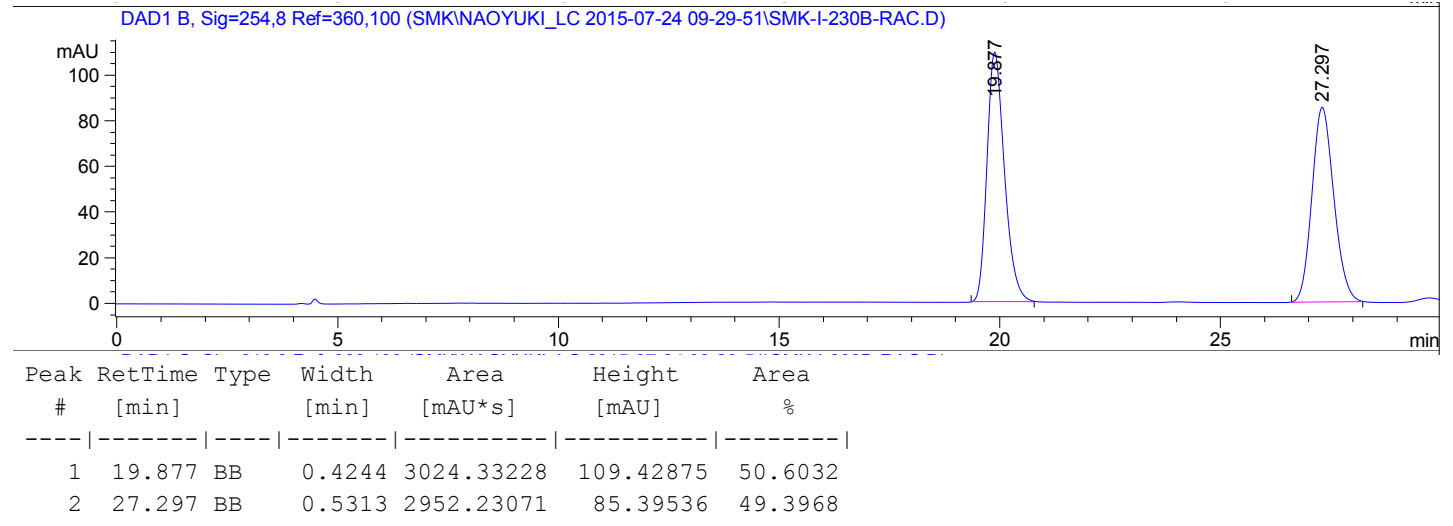




\section{co-injection of DL-6a + DL-Phe-Ot-Bu (5a)}

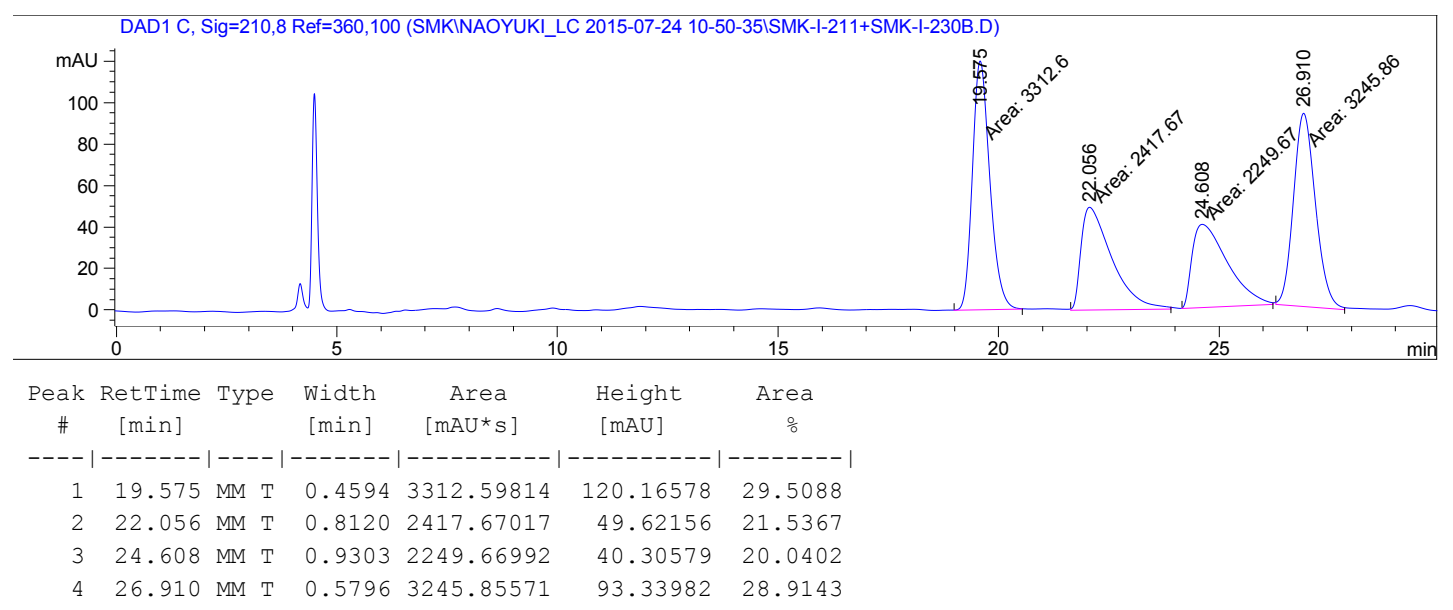

HPLC analysis of crude reaction mixture: L-Phe-O-t-Bu (5a), $81 \%$ ee; L-6a, $97 \%$ ee

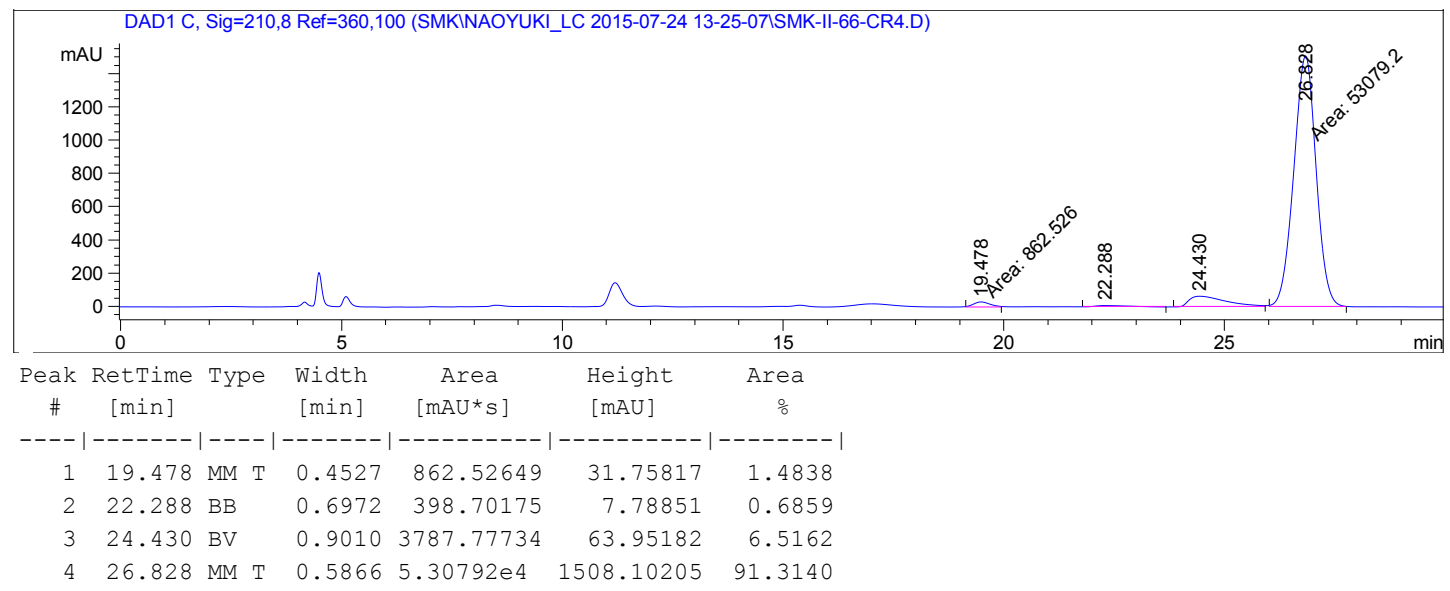

$\mathrm{Ph} \overbrace{\mathrm{NHPh}}^{\mathrm{CO}_{2} t-\mathrm{Bu}}$

$6 a$

L-6a after purification: $97 \%$ ee

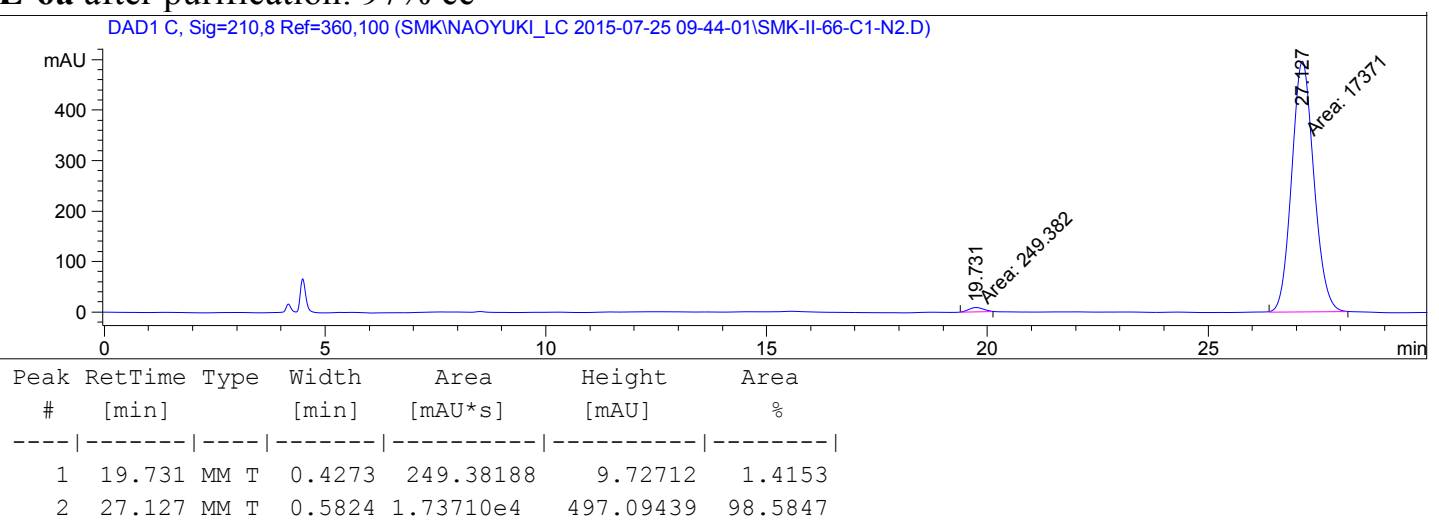


B.

$$
\text { ee before reaction e after reaction }{ }^{a}
$$

\begin{tabular}{ccc}
\hline $\mathbf{9}$ & $93 \%$ & $93 \%$ \\
$\mathbf{5 a}$ & $99 \%$ & $81 \%$ \\
$\mathbf{6 a}$ & - & $93 \%$ \\
\hline${ }^{a}$ Enantiomeric excess (ee) was determined after purification by silica gel chromatography.
\end{tabular}

Scheme 3. B. Experiment to test for product racemization with exogenous and different product added.<smiles>COCc1ccc(NC(Cc2ccccc2)C(=O)OC(C)(C)C)cc1</smiles>

HPLC analysis (OD-H, 10\% IPA-hexanes, $0.8 \mathrm{~mL} / \mathrm{min}, 254 \mathrm{~nm}$ ) indicated 93\% ee: $\mathrm{tR}$ (minor) = $7.8 \mathrm{~min}, \mathrm{tR}$ (major) $=10.6 \mathrm{~min}$.

\section{DL-9}

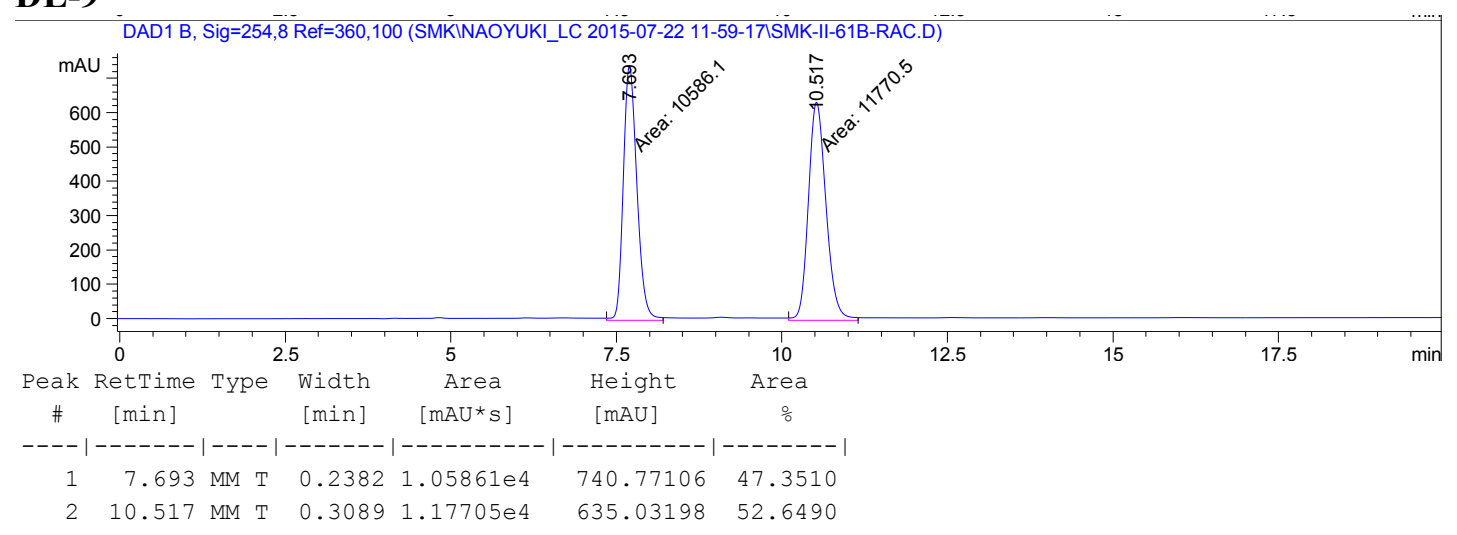

L-9 starting material: $93 \%$ ee

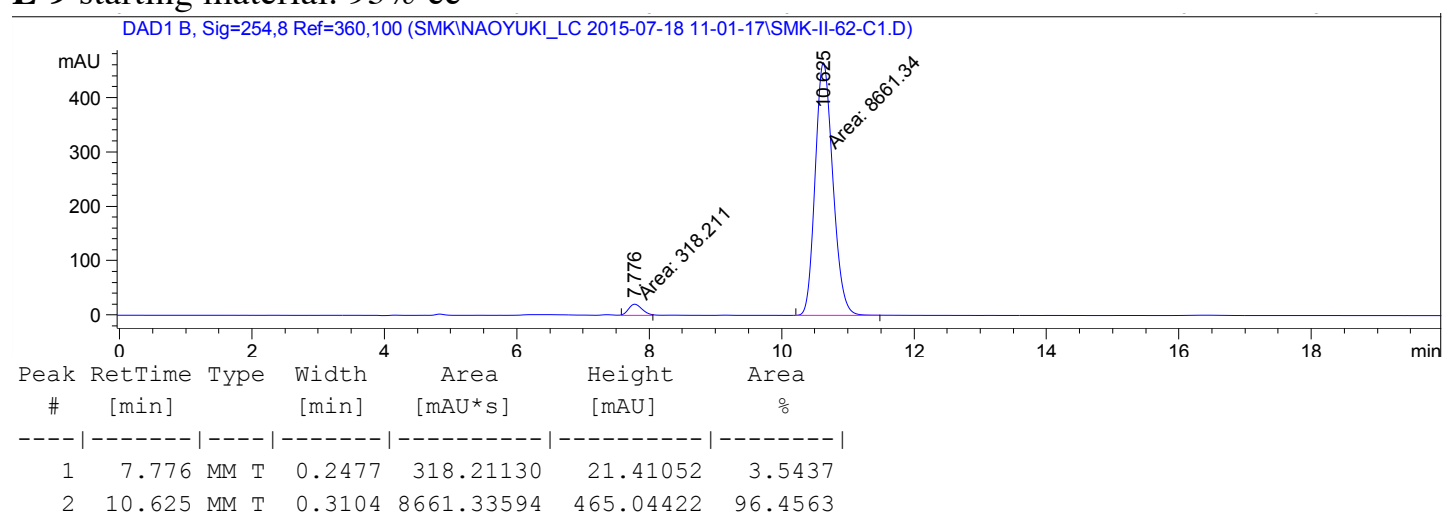


L-9 after purification: 93\% ee

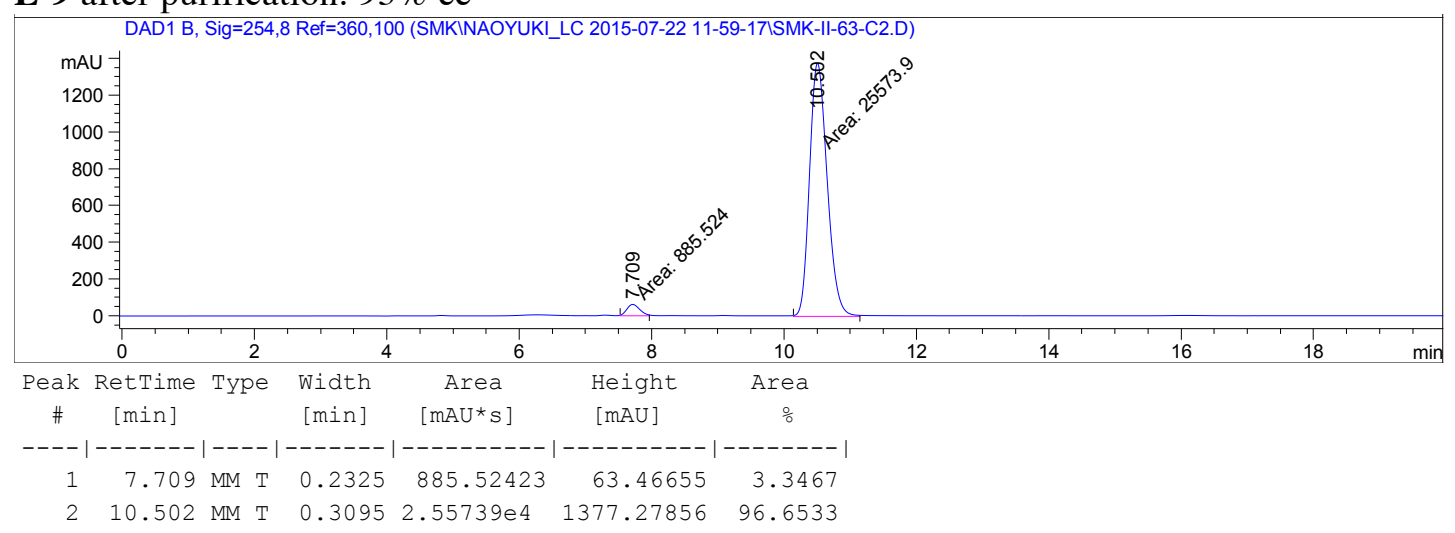

$\mathrm{Ph} \overbrace{\mathrm{NHPh}}^{\mathrm{CO}_{2} t-\mathrm{Bu}}$

$6 a$

HPLC analysis conditions: AD-H, 1\% IPA-hexanes, $0.8 \mathrm{~mL} / \mathrm{min}, 254 \mathrm{~nm}$, tR (minor) $=19.8 \mathrm{~min}$, $\mathrm{tR}($ major $)=26.6 \mathrm{~min}$.

\section{DL-6a}

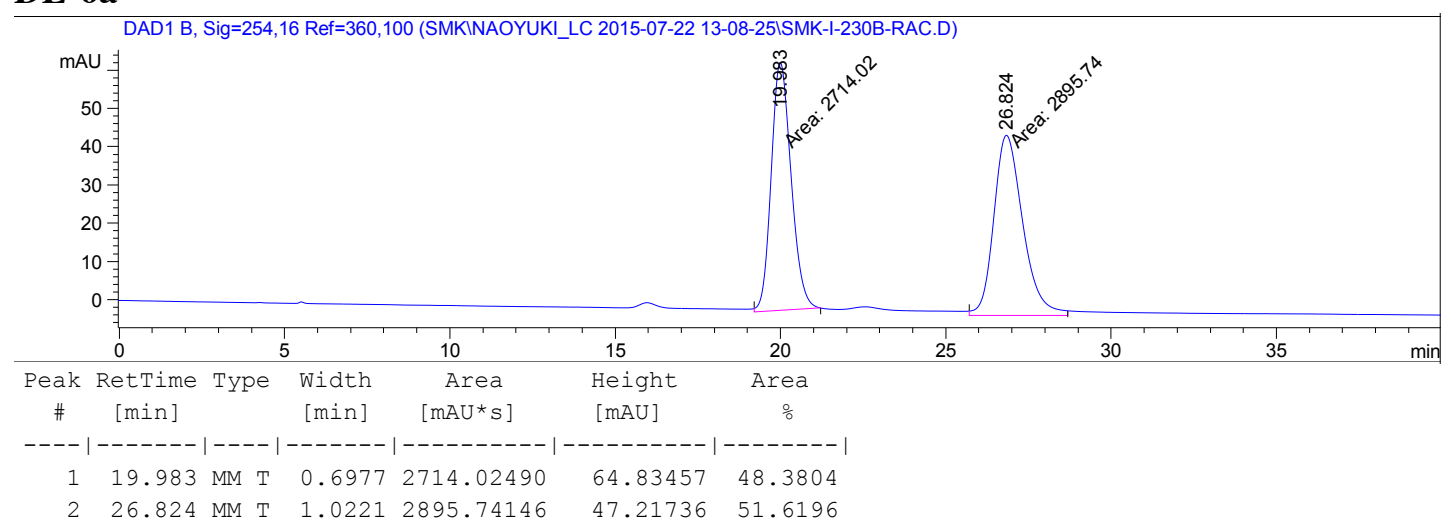

L-6a reaction product after purification: $93 \%$ ee

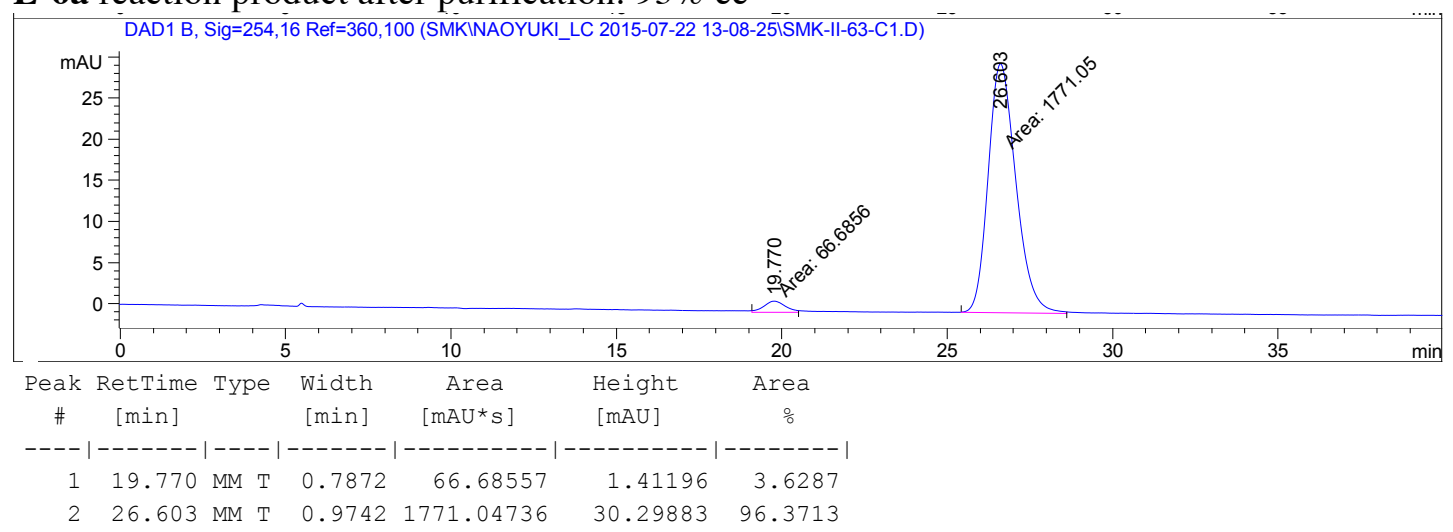


$\overbrace{\text { L-Phe-Ot-Bu (5a) }}^{\mathrm{NH}_{2} t-\mathrm{Bu}}$

HPLC analysis conditions: AD-H, 1\% IPA-hexanes, $0.8 \mathrm{~mL} / \mathrm{min}, 254 \mathrm{~nm}$, tR (minor) = $21.8 \mathrm{~min}$, $\operatorname{tR}($ major $)=23.8 \mathrm{~min}$

\section{DL-Phe-Ot-Bu (5a)}

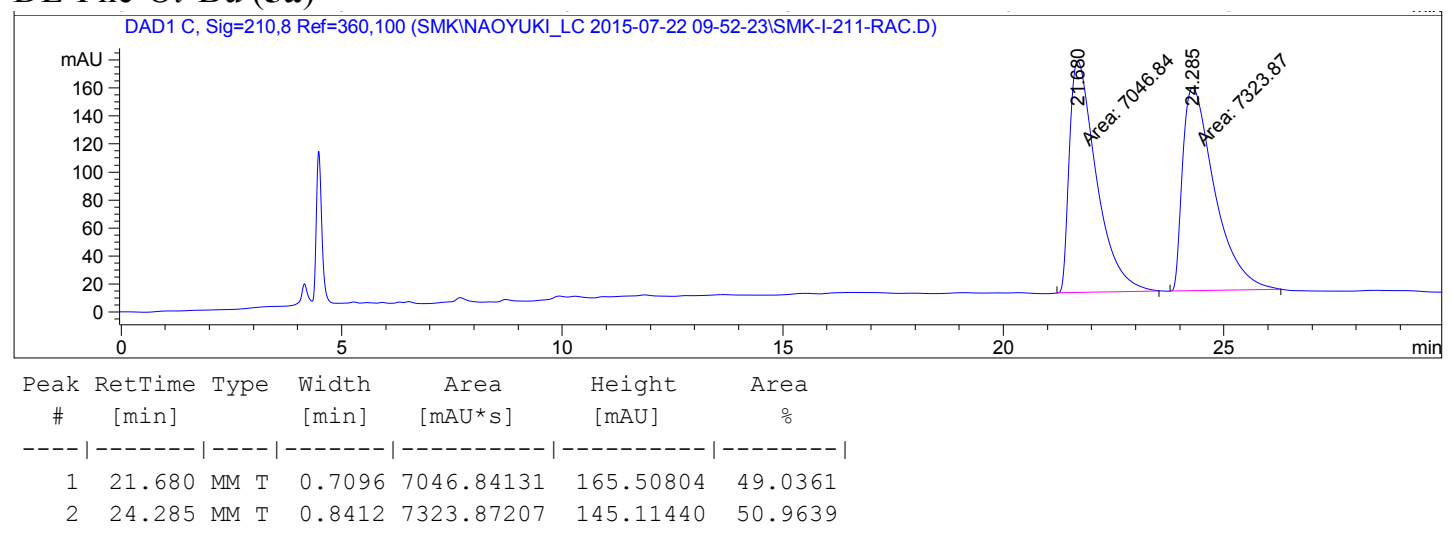

L-Phe-Ot-Bu (5a) starting material: 99\% ee

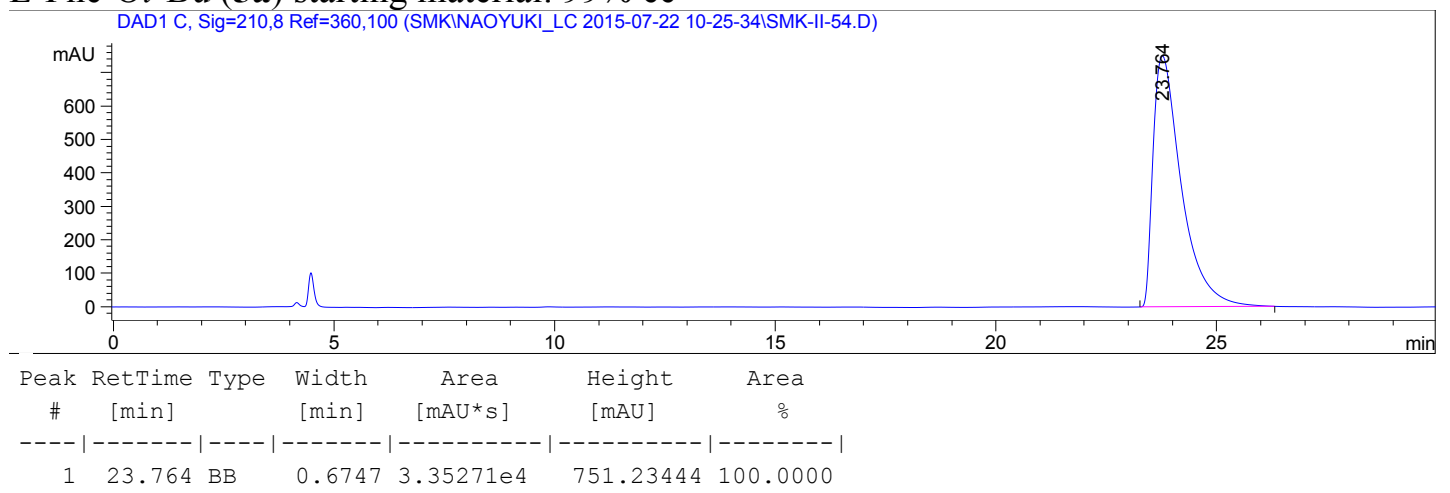

L-Phe-Ot-Bu (5a) after purification: $81 \%$ ee

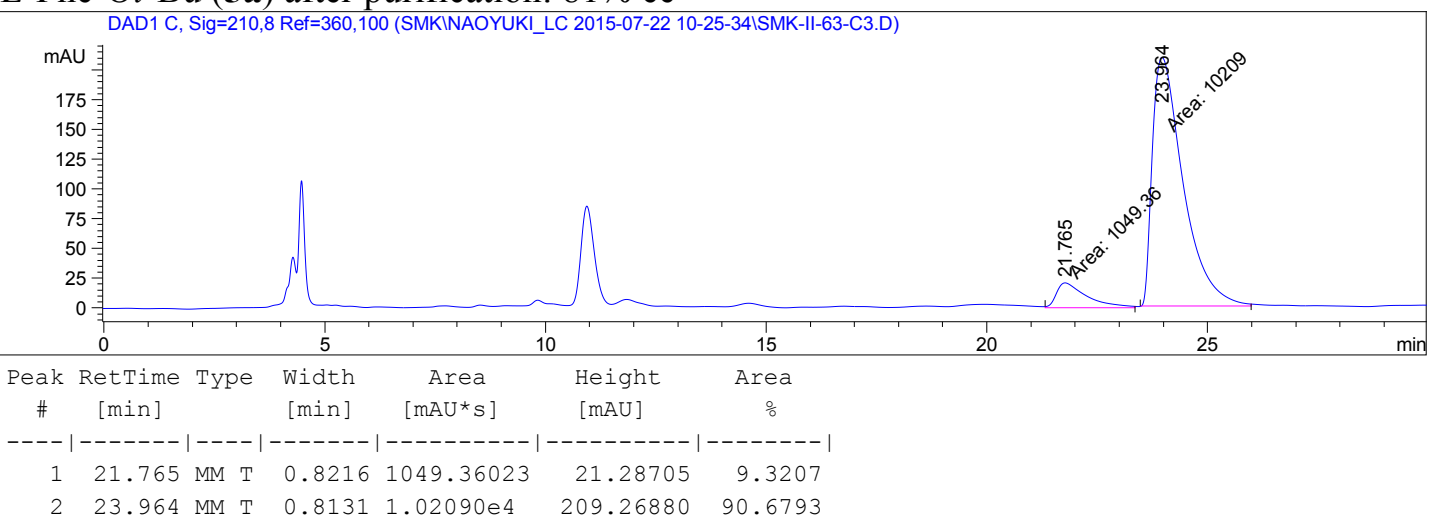




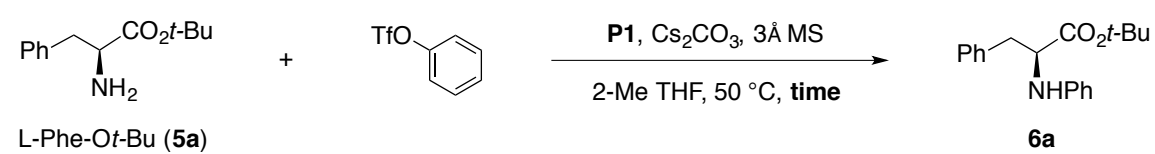

\begin{tabular}{cccc}
\hline \multicolumn{4}{c}{ Table S5. Evaluation of yield and ee over reaction time. } \\
\hline entry & time & yield & ee \\
\hline 1 & $30 \mathrm{~min}$ & $67 \%$ & $98 \%$ \\
2 & $1 \mathrm{~h}$ & $73 \%$ & $97 \%$ \\
3 & $1.5 \mathrm{~h}$ & $90 \%$ & $95 \%$ \\
4 & $2 \mathrm{~h}$ & $92 \%$ & $95 \%$ \\
5 & $4 \mathrm{~h}$ & $96 \%$ & $93 \%$ \\
6 & $16 \mathrm{~h}$ & $99 \%$ & $88 \%$ \\
7 & $10 \mathrm{~d}$ & $91 \%$ & $10 \%$ \\
\hline
\end{tabular}

$\mathrm{Ph} \overbrace{\mathrm{NHPh}}^{\mathrm{CO}_{2} t-\mathrm{Bu}}$

$6 a$

HPLC analysis conditions: OJ-H, 2\% IPA-hexanes, $0.8 \mathrm{~mL} / \mathrm{min}, 254 \mathrm{~nm}$

\section{DL-6a}

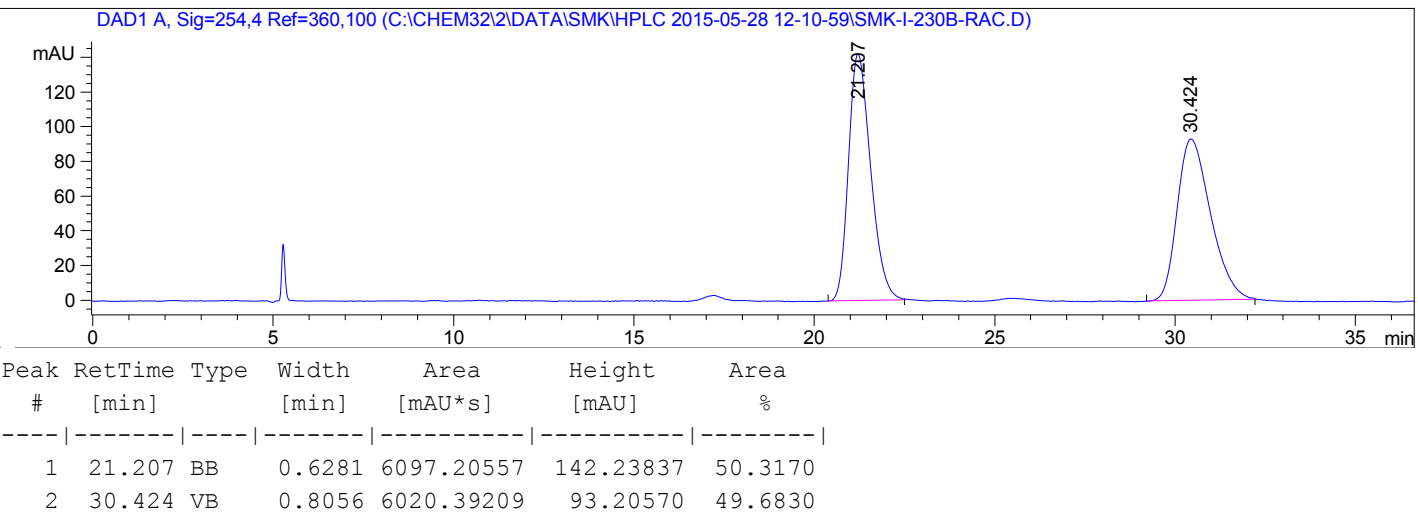

L-6a entry 1: $98 \%$ ee

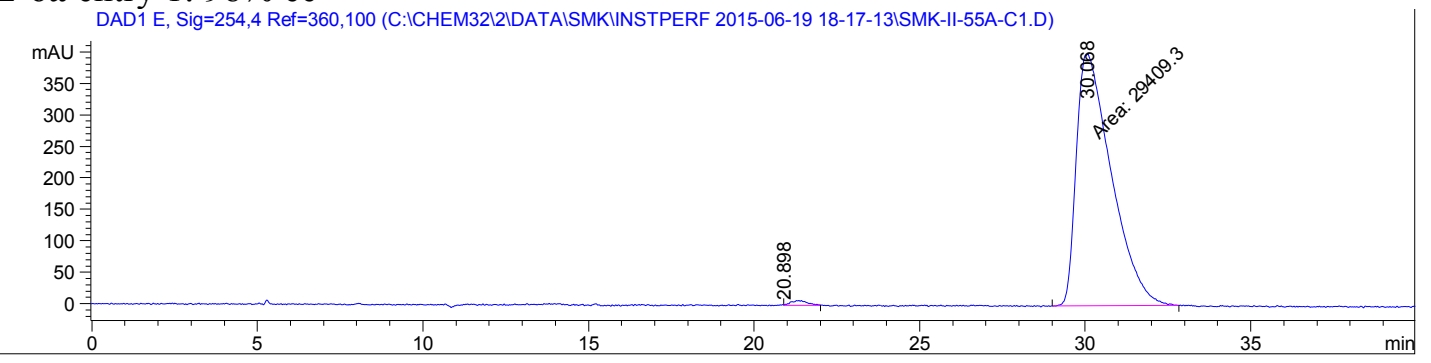




\begin{tabular}{|c|c|c|c|c|c|c|}
\hline $\begin{array}{c}\text { eak } \\
\#\end{array}$ & $\begin{array}{c}\text { RetTime } \\
\text { [min] }\end{array}$ & Type & $\begin{array}{c}\text { Width } \\
\text { [min] }\end{array}$ & $\begin{array}{c}\text { Area } \\
{\left[\mathrm{mAU}^{*} \mathrm{~s}\right]}\end{array}$ & $\begin{array}{l}\text { Height } \\
\text { [mAU] }\end{array}$ & $\begin{array}{c}\text { Area } \\
\frac{\circ}{0}\end{array}$ \\
\hline & --- & & & ---- & $\mid---$ & 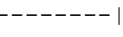 \\
\hline 1 & & & 6 & 277.33759 & $49 e-1$ & 342 \\
\hline$\lambda$ & 68 & 1 & 2234 & $2.94093 e 4$ & 400.64212 & 0658 \\
\hline
\end{tabular}

L-6a entry 2: 97\% ee

DAD1 E, Sig=254,4 Ref=360,100 (C:ICHEM32I2IDATAISMKIINSTPERF 2015-06-19 18-17-13ISMK-II-55B-C1.D)

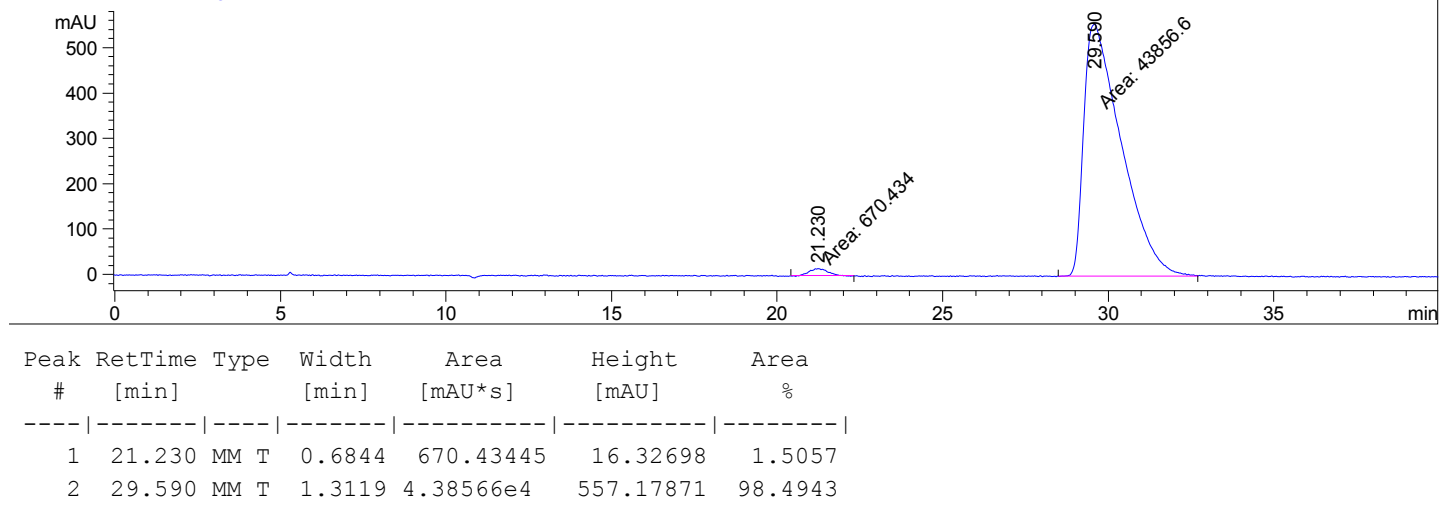

L-6a entry 3: 95\% ee

DAD1 E, Sig=254,4 Ref=360,100 (C:ICHEM32I2IDATAISMKIINSTPERF 2015-06-19 18-17-13ISMK-II-55C-C1.D)

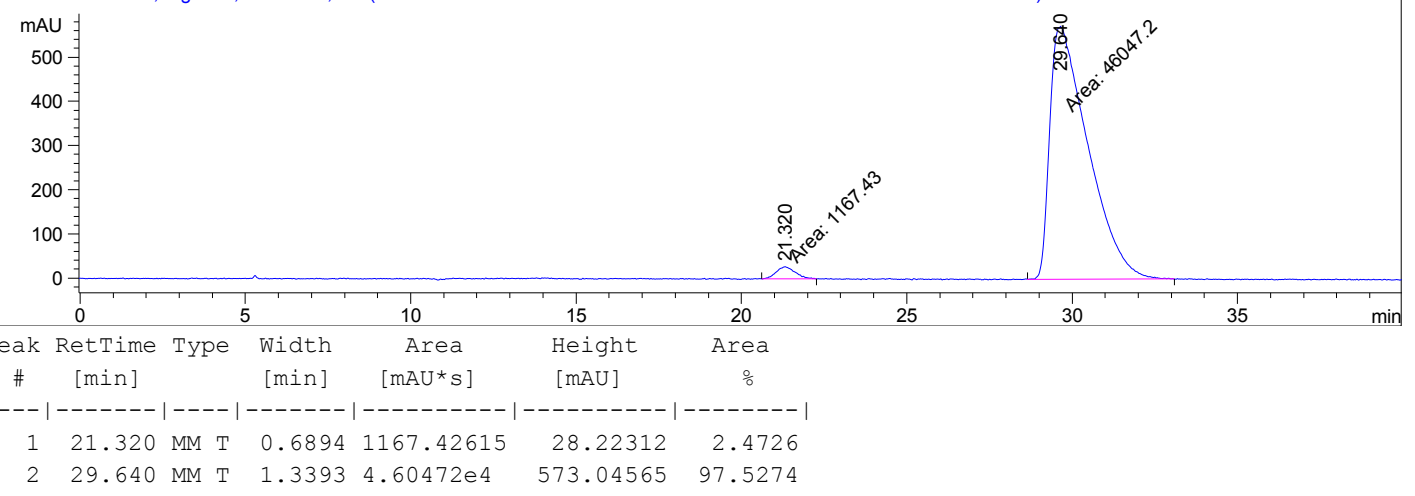

L-6a entry 4: 95\% ee

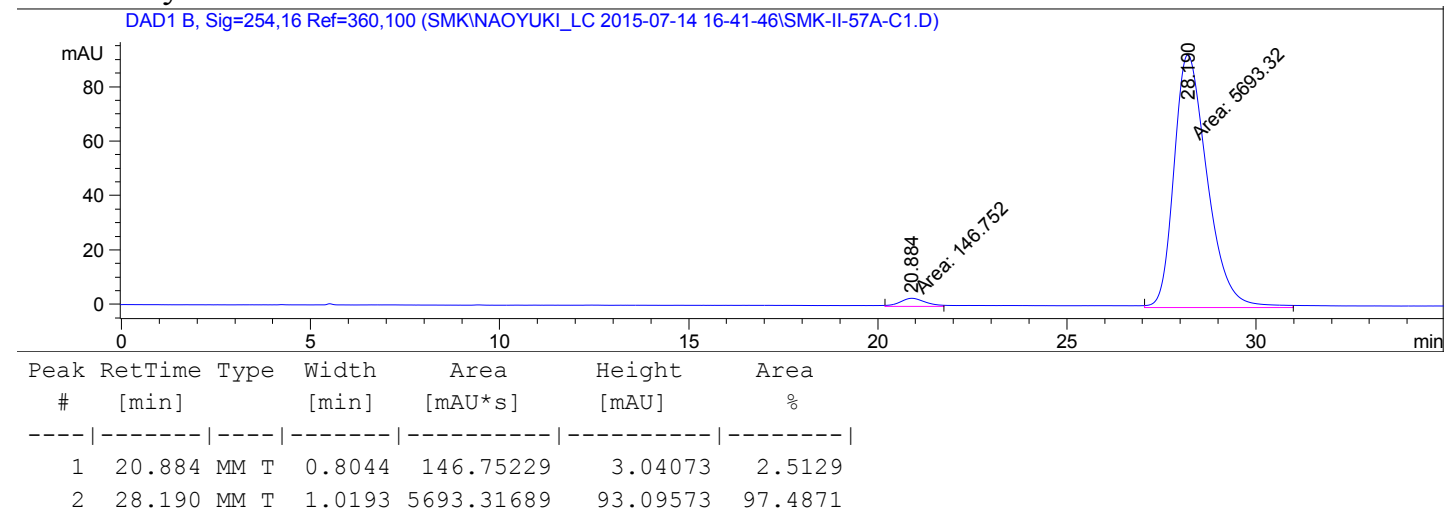


L-6a entry 5: 93\% ee

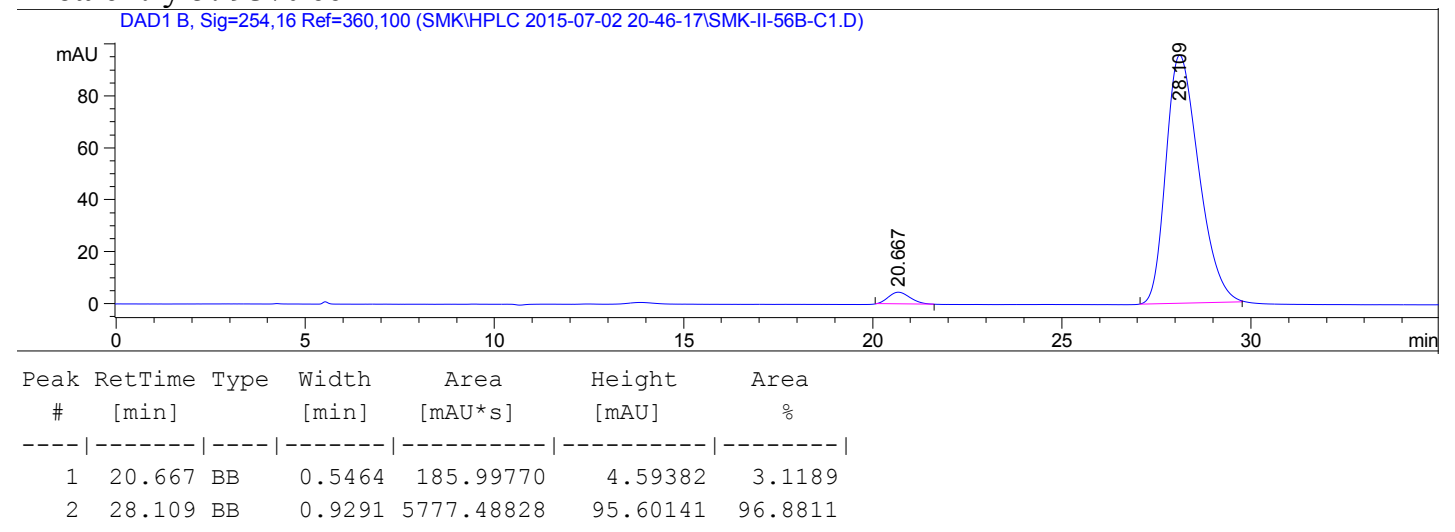

L-6a entry 6: 88\% ee

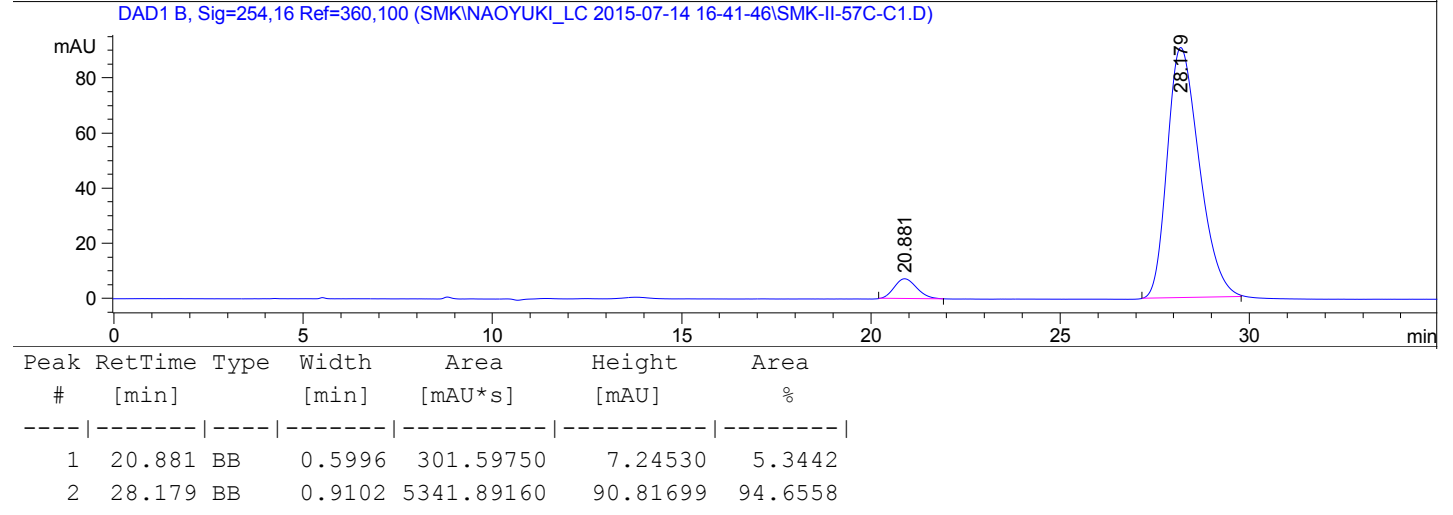

L-6a entry 7: 10\% ee

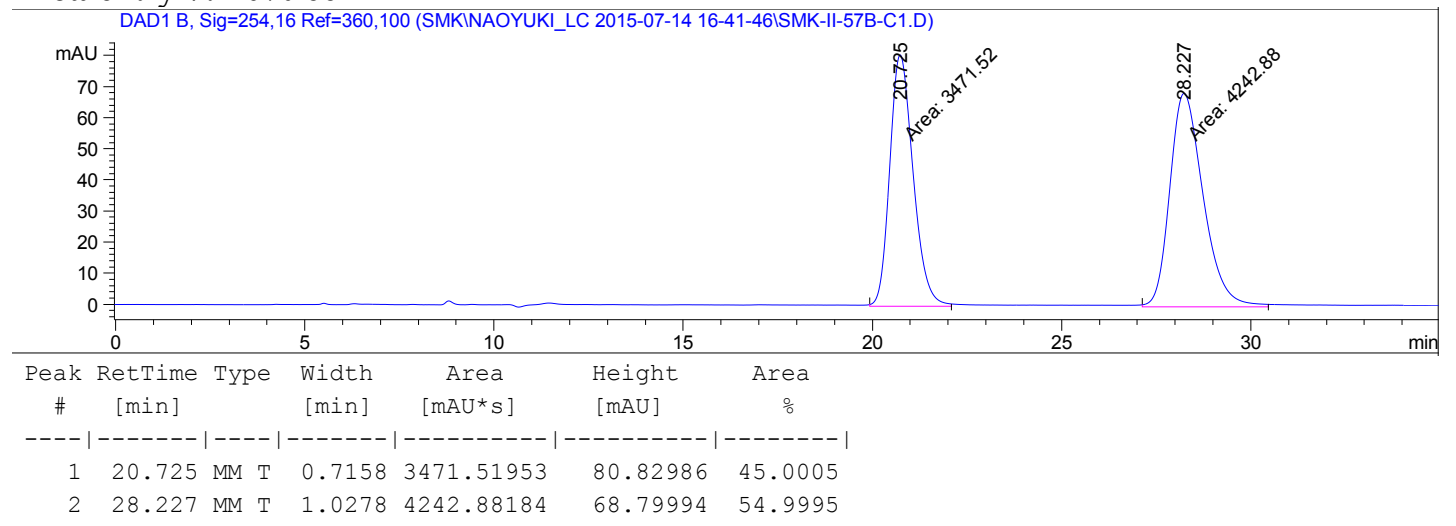




\section{Bibliography}

1. Bruno, N. C.; Niljianskul, N.; Buchwald, S. L., J. Org. Chem. 2014, 79, 4161.

2. $\quad$ Bruno, N. C.; Buchwald, S. L., Org. Lett. 2013, 15, 2876.

3. Abato, P.; Yuen, C. M.; Cubanski, J. Y.; Seto, C. T., J. Org. Chem. 2002, 67, 1184.

4. Hughes, M.; Birchall, L. S.; Zuberi, K.; Aitken, L. A.; Debnath, S.; Javid, N.; Ulijn, R. V., Soft Matter 2012, 8, 11565.

5. Zhu, Y.; Wu, G.; Zhu, X.; Ma, Y.; Zhao, X.; Li, Y.; Yuan, Y.; Yang, J.; Yu, S.; Shao, F.; Lei, M., J. Med. Chem. 2010, 53, 8619.

6. Milner, P. J.; Kinzel, T.; Zhang, Y.; Buchwald, S. L., J. Am. Chem. Soc. 2014, 136, 15757.

7. Petrassi, H. M.; Klabunde, T.; Sacchettini, J.; Kelly, J. W., J. Am. Chem. Soc. 2000, 122, 2178.

8. Mori, A.; Mizusaki, T.; Ikawa, T.; Maegawa, T.; Monguchi, Y.; Sajiki, H., Chem. - Eur. J. 2007, 13, 1432.

9. $\quad$ Kale, A. P.; Pawar, G. G.; Kapur, M., Org. Lett. 2012, 14, 1808.

10. Brady, R. M.; Vom, A.; Roy, M. J.; Toovey, N.; Smith, B. J.; Moss, R. M.; Hatzis, E.; Huang, D. C. S.; Parisot, J. P.; Yang, H.; Street, I. P.; Colman, P. M.; Czabotar, P. E.; Baell, J. B.; Lessene, G., J. Med. Chem. 2014, 57, 1323.

11. Proutiere, F.; Schoenebeck, F., Angew. Chem., Int. Ed. Engl. 2011, 50, 8192.

12. Goossen, L. J.; Rodríguez, N.; Linder, C., J. Am. Chem. Soc. 2008, 130, 15248.

13. Lee, H. G.; Milner, P. J.; Buchwald, S. L., Org. Lett. 2013, 15, 5602.

14. Furuya, T.; Strom, A. E.; Ritter, T., J. Am. Chem. Soc. 2009, 131, 1662.

15. Guptill, D. M.; Davies, H. M. L., J. Am. Chem. Soc. 2014, 136, 17718.

16. For clarity synthetic intermediates not described in the manuscript are numbered in the Supporting Information beginning with S1. 